\title{
The development of an experimental model to assess mechanical performance of skeletal muscle in the intact mouse
}

Citation for published version (APA):

Gorselink, M. (2001). The development of an experimental model to assess mechanical performance of skeletal muscle in the intact mouse. [Doctoral Thesis, Maastricht University]. Universiteit Maastricht. https://doi.org/10.26481/dis.20010628mg

Document status and date:

Published: 01/01/2001

DOI:

10.26481/dis.20010628mg

Document Version:

Publisher's PDF, also known as Version of record

Please check the document version of this publication:

- A submitted manuscript is the version of the article upon submission and before peer-review. There can be important differences between the submitted version and the official published version of record.

People interested in the research are advised to contact the author for the final version of the publication, or visit the DOI to the publisher's website.

- The final author version and the galley proof are versions of the publication after peer review.

- The final published version features the final layout of the paper including the volume, issue and page numbers.

Link to publication

\footnotetext{
General rights rights.

- You may freely distribute the URL identifying the publication in the public portal. please follow below link for the End User Agreement:

www.umlib.nl/taverne-license

Take down policy

If you believe that this document breaches copyright please contact us at:

repository@maastrichtuniversity.nl

providing details and we will investigate your claim.
}

Copyright and moral rights for the publications made accessible in the public portal are retained by the authors and/or other copyright owners and it is a condition of accessing publications that users recognise and abide by the legal requirements associated with these

- Users may download and print one copy of any publication from the public portal for the purpose of private study or research.

- You may not further distribute the material or use it for any profit-making activity or commercial gain

If the publication is distributed under the terms of Article $25 \mathrm{fa}$ of the Dutch Copyright Act, indicated by the "Taverne" license above, 
The development of an experimental model to assess mechanical performance of skeletal muscle in the intact mouse 
The studies presented in this thesis were performed at the Cardiovascular Research Institute Maastricht (CARIM), University Maastricht, The Netherlands in corroboration with Biomedical Technology, Technical University Eindhoven, The Netherlands.

(C) 2001 Marchel Gorselink ISBN 90-9014829-9

Lay-out M. Gorselink, Ede, The Netherlands

Cover design Design Arbeid, Amsterdam, The Netherlands

Printed by GSC Van Gils, Wageningen, The Netherlands

Printing of this thesis was fincially supported by Nutricia Nederland, B.V., Zoetermeer, The Netherlands and Stichting Swoof, Maastricht, The Netherlands. 
The development of an experimental model to assess mechanical performance of skeletal muscle in the intact mouse

\section{PROEFSCHRIFT}

ter verkrijging van de graad van doctor aan de Universiteit Maastricht, op gezag van de Rector Magnificus, Prof. dr. A.C. Nieuwenhuijzen Kruseman, volgens het besluit van het College van Decanen, in het openbaar te verdedigen op donderdag 28 juni, 2001 om 12.00 uur

door

\section{Marchel Gorselink}

Geboren te Epe op 24 juni 1972 


\section{Promotores:}

Prof. dr. G.J. van der Vusse

Prof. dr. ir. J.D. Janssen

\section{Co-Promotor:}

Dr. ir. M.R. Drost

\section{Beoordelingscommissie:}

Prof. dr. H. Kuipers

Prof. dr. ir. M.G.J. Arts

Dr. A. J. M. Wagenmakers

Prof. dr. B. Wieringa

Dr. ir. W. C. Oomens
(Technische Universiteit Eindhoven)

$$
\text { (voorzitter) }
$$

(Katholieke Universiteit Nijmegen)

(Technische Universiteit Eindhoven) 


\section{Table of Contents}

Chapter 1 INTRODUCTION

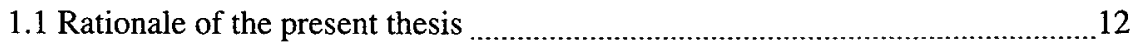

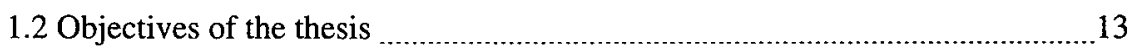

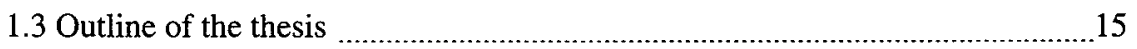

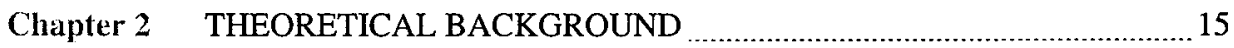

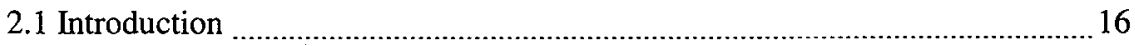

2.2 Skeletal muscle structure

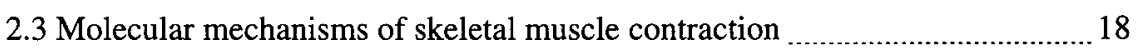

2.4 Functional characteristics of skeletal muscle 20

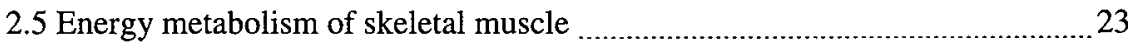

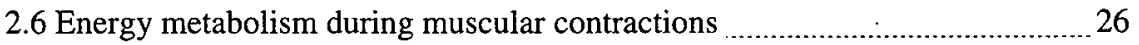

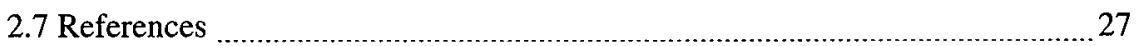

Chapter 3 ACCURATE ASSESSMENT OF IN SITU ISOMETRIC CONTRACTILE PROPERTIES OF HINDLIMB PLANTAR AND DORSAL FLEXOR MUSCLE COMPLEX OF INTACT MICE ….......................... 29

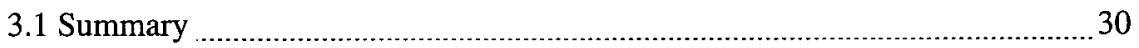

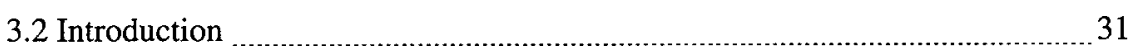

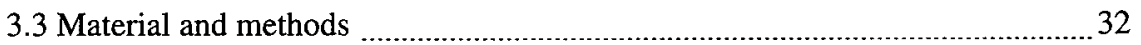

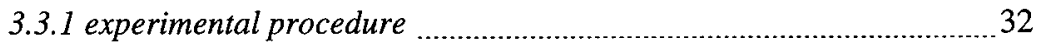

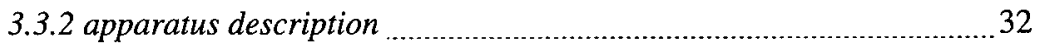

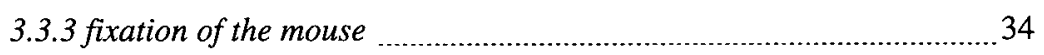

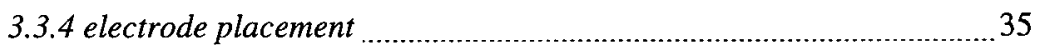

3.3.5 experimental procedure to validate the fixation system ..................... 35

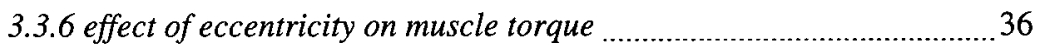

3.3.7 determination of contractile muscle parameters ............................... 36

3.4 Results and discussion

3.4.1 calibration of measurement apparatus ................................................ 38

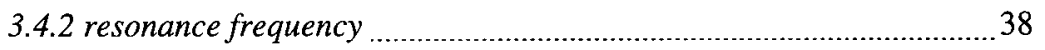

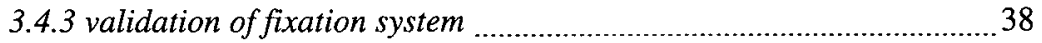

3.4.4 effect of eccentricity on measured torque ........................................... 39

3.4.5 determination of contractile muscle parameters ............................... 40

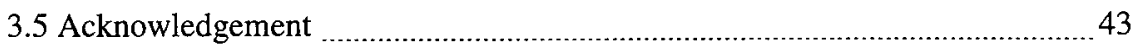

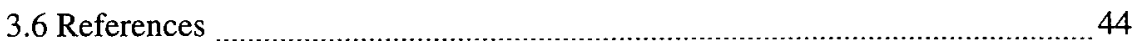


Chapter 4 IN SITU ASSESSMENT OF SHORTENING AND LENGTHENING CONTRACTION PROPERTIES OF HINDLIMB

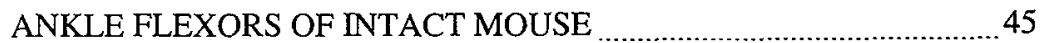

4.1 Summary

4.2 Introduction

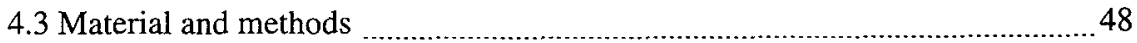

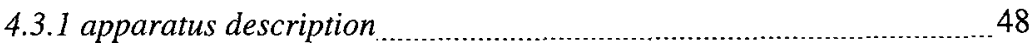

4.3.2 animal handling and muscle complex stimulation ........................... 51

4.3.3 experimental procedure to validate the fixation system ..................... 52

4.3.4 experimental procedure to assess the effect of eccentricity ................. 53

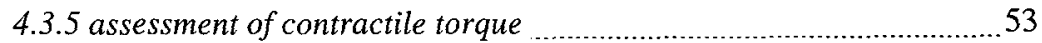

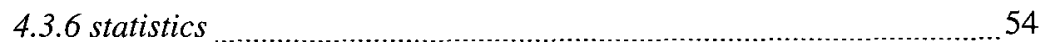

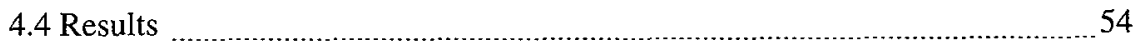

4.4.1 description and evaluation of measurement device ......................... 54

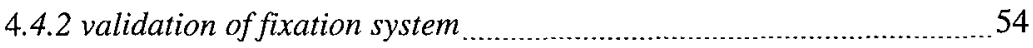

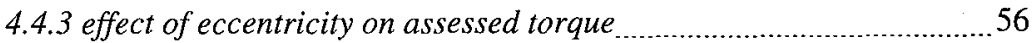

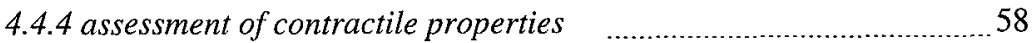

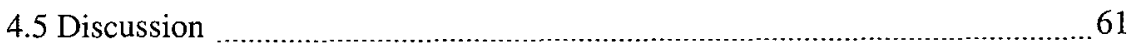

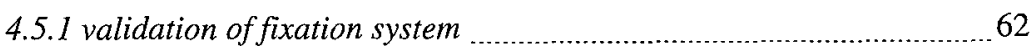

4.5 .2 effects of eccentricity on measured torque .......................................62 62

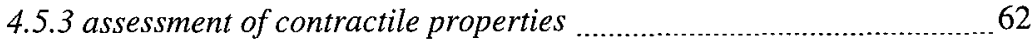

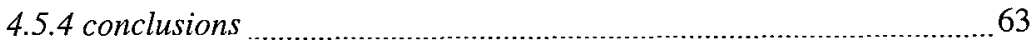

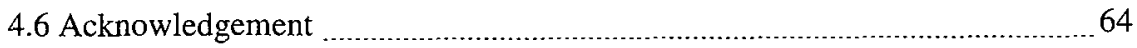

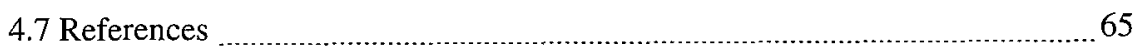

Chapter 5 IMPAIRED MUSCULAR CONTRACTILE PERFORMANCE

AND ADENINE NUCLEOTIDE HANDLING IN

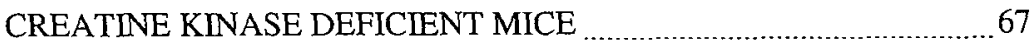

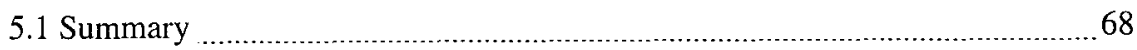

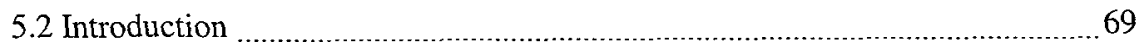

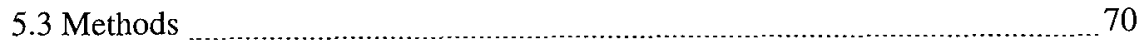

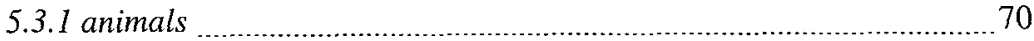

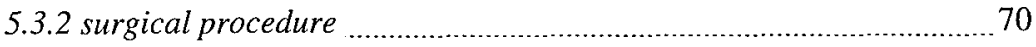

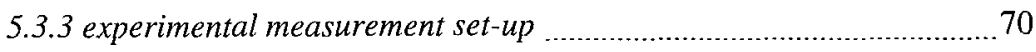

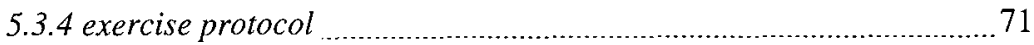

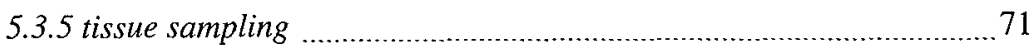

5.3.6 number of animals and statistical procedure .................................. 72

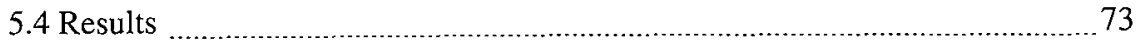




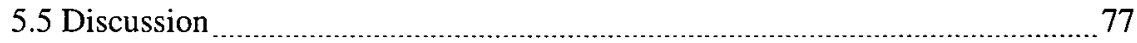

5.5.1 single isometric tetanic contractions ………………..................... 78

5.5.2 repetitive tetanic contraction ……............................................ 79

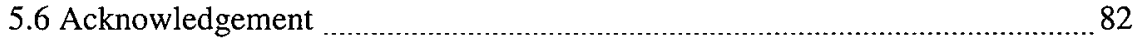

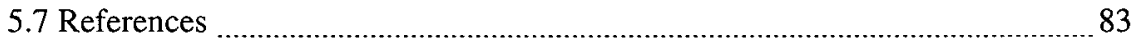

Chapter 6 MURINE MUSCLES DEFICIENT IN CREATINE KINASE TOLERATE CONSECUTIVE SERIES OF HIGH-INTENSITY CONTRACTIONS

6.1 Summary

6.2 Introduction $\quad 87$

6.3 Methods $\quad$.

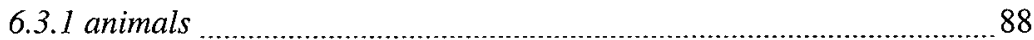

6.3 .2 surgical procedure and stimualtion of the nerve …….................... 88

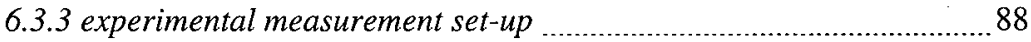

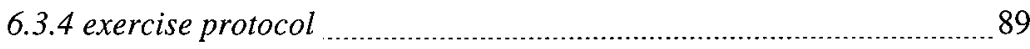

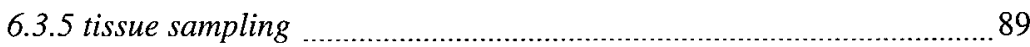

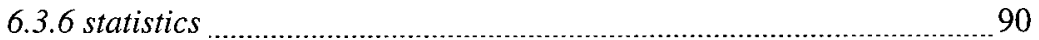

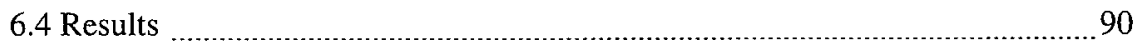

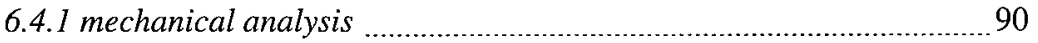

6.4.2 muscle high-energy phosphate content …………........................ 93

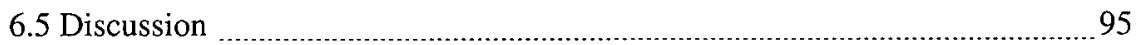

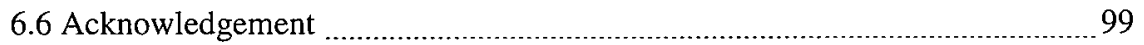

6.7 References $\ldots \ldots \ldots$

Chapter 7 DIFFERENTIAL RESPONSE TO CREATINE KINASE ABLATION BETWEEN DORSAL AND PLANTAR FLEXORS

IN MICE

7.1 Summary $\ldots \ldots$

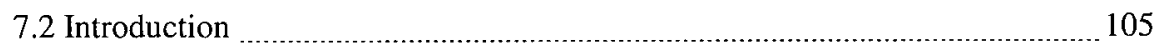

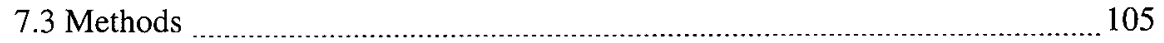

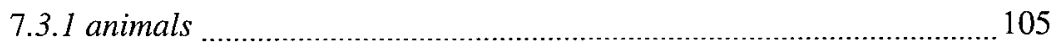

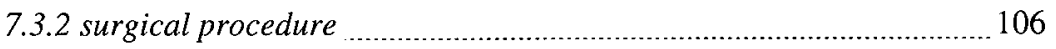

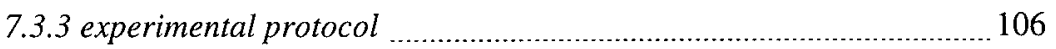

7.3.5 tissue sampling and biochemical analysis _...................................... 107

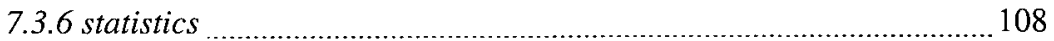

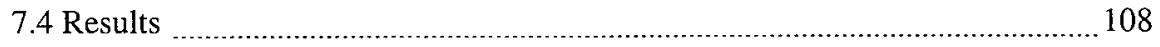


7.5 Discussion

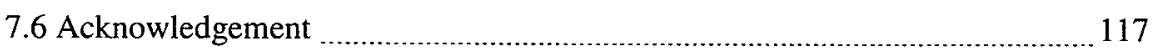

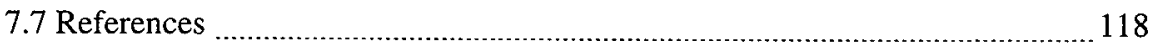

Chapter 8 INCREASED MUSCLE FATIGABILITY IN GLUT4-DEFICIENT MICE

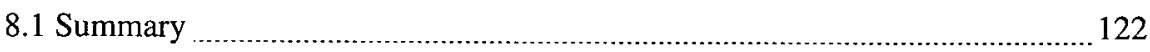

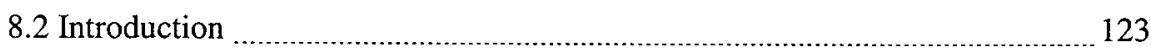

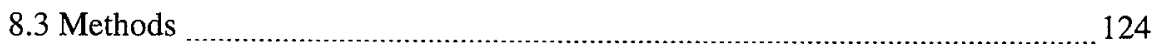

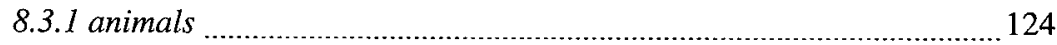

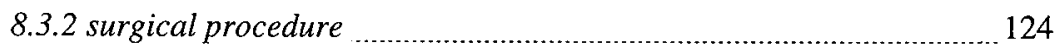

8.3.3 experimental model ……

8.3.4 fatigue protocol $\ldots$

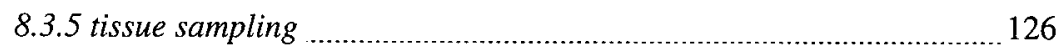

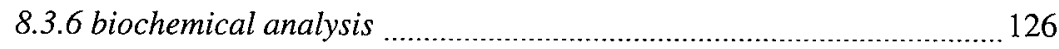

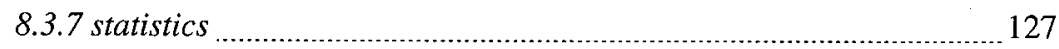

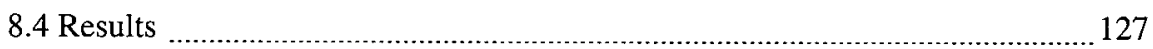

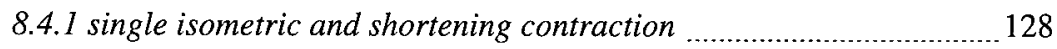

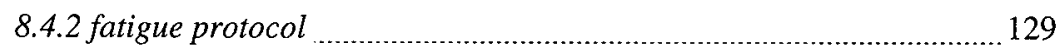

8.4.3 glycogen and high-energy phosphate content ................................ 130

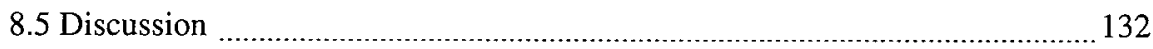

8.5.1 single isometric tetanic contraction .............................................. 132

8.5.2 muscular performance during shortening contractions ..................... 133

8.5.2 fatigue protocol ............................................................................ 133

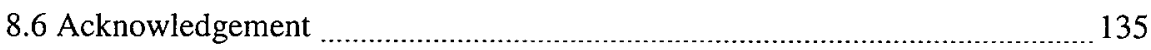

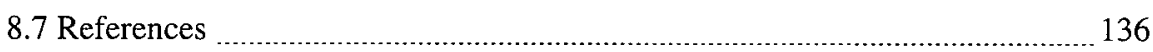

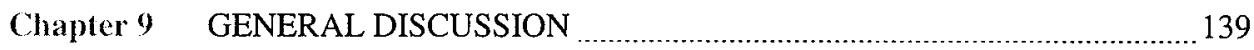

9.1 Study objectives and experimental approach .................................................. 140

9.2 Assessment of mechanical functioning of the intact murine muscle ................ 140

9.3 Role of creatine kinase in muscle mechanical performance .............................. 143

9.4 Role of GLUT4 in muscular mechanical performance ….............................. 147

9.5 Future perspectives _ _ 148

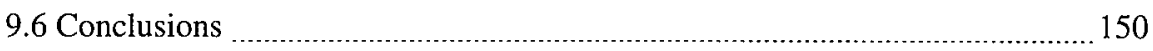

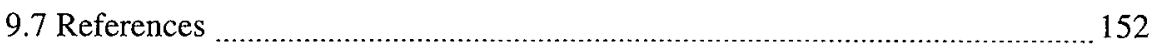




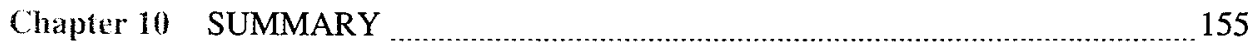

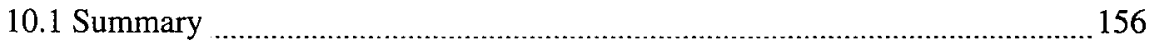

10.2 References

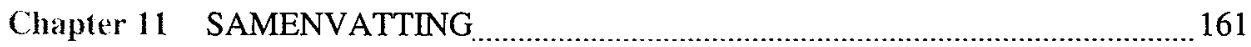

11.1 Samenvatting $\quad 162$

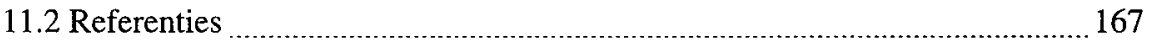

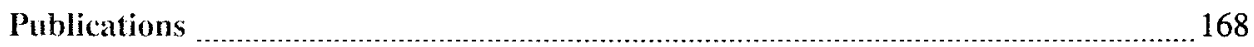

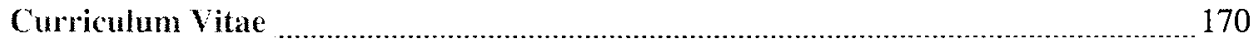

Dankwoord 171 


\section{Chapter 1}

NTRODUCTION

Formulation of the research objectives and a brief description of the thesis content. 


\section{1 - Rationale of the present thesis}

Skeletal muscle comprises about $40 \%$ of the whole body mass, and plays an important role in locomotion. Animal models, like the rat and the mouse, are often used in studying muscular functioning and energy metabolism since experiments on these species are more easily to perform and to standardise compared to human studies on muscular functioning. Recently, the generation of transgenic and knockout mice has greatly enhanced the possibilities for studying the specific role of proteins and enzymes in energy metabolism and mechanical functioning of the skeletal muscle. Muscle contractile properties in mice, however, are mostly studied using invasive techniques, extending from single muscle fibre studies to whole muscle experiments. In these experimental models, the selective fibres or muscles of interest are dissected from their natural surrounding and attached to a force transducer. Muscular contractility is studied via electrical stimulation of the muscle or its nerve. Consequently, these models restrict the investigator to acute experiments, and analysis of the long-term effects of a specific intervention, using the animal as its own control, is not possible. Moreover, the surgical procedure of dissecting the muscle or fibres often results in damage of the tissue, i.e., injury of the blood vessels, nerves, or the muscle itself. This invasive approach in studying skeletal muscle functioning in the mouse may lead to misinterpretations, when extrapolating the results towards the in vivo situations. Therefore, we wanted to develop an experimental model with which mechanical characteristics of skeletal muscle of the mouse can be investigated under physiological conditions as much as possible.

The universal energy donor in the skeletal muscle cell for energy utilising processes, such as a variety of ion pumps and distinct steps in metabolic pathways, and the contractile machinery itself, is adenosine triphosphate (ATP). There are several energy producing systems to replenish the ATP pool. For instance, ATP is generated from phosphocreatine ( $\mathrm{PCr}$ ), an additional high-energy phosphate pool in the sarcoplasm by the catalytic action of creatine kinase (CK). These high-energy phosphate pools, however, are limited and must constantly be replenished via the metabolic conversion of carbohydrates and fatty acids. Carbohydrates are taken up from the blood compartment mainly as glucose. Specific glucose transporters, such as GLUT4, are present in the muscular cell to facilitate trans-sarcolemmal transport of glucose. Two pathways are involved in the metabolism of glucose, i.e., anaerobic and aerobic. The anaerobic process occurs in the cytoplasm involving the conversion of glucose to lactate, and produces a moderate amount of ATP. The aerobic combustion takes place in the mitochondria and generates a substantial amount of ATP. Moreover, the aerobic combustion of fatty acids via the $\beta$-oxidation is also a major source of ATP. 
Many proteins either transport proteins or enzymes are involved in these metabolic processes, and their absence or defects in the biological function lead to complications in energy metabolism, which may affect the contractile behaviour of the muscle.

Muscular contractility depends, among others, on the nature of the contraction. Skeletal muscle contraction can be divided into three types of contraction, i.e., isometric, concentric and eccentric contractions. During isometric contractions, the length of the muscle complex remains constant, whereas during concentric and eccentric the length of the muscle complex respectively shortens and lengthens. Assessment of the properties of these types of contractions requires specific measurement devices either for isometric or dynamic contraction characteristics. To study the muscle under physiological conditions, the muscle complex of interest should be left in its natural surrounding. Muscular performance also depends on fibre type composition. Therefore, we have chosen to study two muscle complexes around the ankle joint, i.e., dorsal flexor complex and the plantar flexor complex. The dorsal flexor complex comprises the mainly fast-twitch tibialis anterior muscle and the extensor digitorum muscle, whereas the plantar flexor complex consists of the fast twitch gastrocnemius and plantaris muscles and slow-twitch soleus muscle.

\section{2 - Objectives of the thesis}

The first objective of the present thesis is developing an experimental model to determine the mechanical performance of the intact dorsal and plantar flexors of the mouse. The experimental model must allow for accurate assessment of both isometric and shortening/lengthening contractile properties of the murine skeletal muscle. As a second objective, we aimed to study the consequence of defects in energy metabolism on mechanical functioning of murine skeletal muscle. First, we studied the effect of disruption of the cytoplasmic and mitochondrial creatine kinase gene on muscular performance. Creatine kinase forms a family of iso-enzymes playing an important role in maintaining the levels of ATP and PCr in muscle cells. Purpose of this thesis is to delineate the functional impact of the lack of CK activity on muscular contractility and high-energy phosphate handling. Second, we investigated whether disruption of the glucose transporter (GLUT4) gene affects isometric and shortening contractile performance of the dorsal flexor muscle complex in situ. Moreover, we have explored the hypothesis that the lack of GLUT4 enhances muscular fatigability. 


\section{3 - Outline of the thesis}

After a short introduction (Chapter 1), the thesis starts with a general introduction into skeletal muscle functioning, anatomy and energy metabolism (Chapter 2). Chapters 3 and 4 give a detailed description of the developed experimental model for assessment of the contractile properties under isometric as well as under dynamic conditions of the dorsal and plantar muscle complexes of the intact mouse. Isometric contractile performance of both muscle complexes can be assessed with the isometric mouse dynamometer (Chapter 3). A detailed description of the measurement device for accurate assessment of the isometric contractile properties is given. Special attention is paid to a novel fixation method of the mouse hindlimb to the measurement device. Moreover, it is verified whether eccentricity, i.e., misalignment of ankle joint and the measurement axis, affects the contractility of the muscle. Shortening and lengthening properties of the dorsal and plantar flexor complex can be studied with the mouse ergometer (Chapter 4). In this chapter, the fixation technique was validated, and representative examples of shortening and lengthening contractions of both muscle complexes are shown. Moreover, dynamic properties of the muscle complexes are described in terms of peak power and the optimal and maximal shortening velocities of wild type mice.

Chapter 5 and 6 give a detailed description of the effect of absence in mitochondrial and cytoplasmic creatine kinase on isometric mechanical functioning of the dorsal flexor complex. The effect of creatine kinase deficiency on muscular contractility and on levels of high-energy phosphates was determined during an intermittent contraction series of high-intensity. Furthermore, a comparison in mechanical functioning between the dorsal and plantar flexor complex in creatine kinase deficient mice was made (Chapter 7). The next chapter reveals the effects of the deficiency of a muscular glucose transporter (GLUT4) during a single isometric and shortening contraction (Chapter 8). The degree of fatigability was also assessed in GLUT4 knock out and wild type mice during a contraction series of moderate intensity.

The thesis ends with a general discussion, which summarises the major findings in the preceding chapters, with special reference to the assessment of muscular performance in the intact mouse (Chapter 9). 


\section{Chapter 2}

\section{Theoretical background}

Based on: M. Gorselink, M.R. Drost, and G.J. van der Vusse. (2001) Muscle morphology, metabolism and muscle function. General principles, Nutrition and metabolism in Respiratory Diseases, Rossi, Verona, in press.

A general introduction into skeletal muscle functioning, anatomy and energy metabolism is provided. 


\section{1 - Introduction}

Skeletal muscles are designed to match the functional demands of daily life. They have the ability to both accurately initiate and counteract movements of the body segments, by generating forces and thus moments across the joints. Skeletal muscles are able to deliver their force output over a range of muscle lengths with a variety of shortening and lengthening velocities. To fulfil these specific tasks, skeletal muscle does consist of a great number of interacting force-generating filaments, which are controlled and regulated via a variety of metabolic and neurological processes. To be able to understand muscular functionality, one must understand the highly organised structure of the skeletal muscle. To become familiar with functional and metabolic aspects of muscular contraction, this chapter gives a brief overview of structural, mechanical and metabolic properties of skeletal muscle.

\section{2 - Skeletal Muscle Structure}

In general, the skeletal muscle complex can be divided in active force producing structures (contractile elements) and passive structures. The passive structures are arranged in series, e.g. tendons or aponeuroses, and in parallel with the contractile elements, such as parts of the cytoskeleton and extracellular matrix (1). The active elements play an essential role in force production, whereas the passive elements play an important role in force transmission and in maintaining the structure of the muscle complex.

Skeletal muscle is surrounded by a thick outer connective tissue, called the epimysium. The perimysium divides the muscle further into bundles (fascicles), and provides the pathway for the blood vessels, e.g. arterioles and venules, and nerves through the muscle. The individual muscle cells, also referred to as muscle fibres, are located within these bundles and are surrounded by a thinner connective tissue sheet (endomysium). The muscle complex contains distinct types of fibres, and the properties of which vary within a continuum. For simplicity, muscle fibres can be classified as fast and slow twitch fibres. Slow twitch fibres (I) have low power output and slow maximal speed of shortening, and are equipped for endurance contraction series. Fast twitch fibres have high power output and high shortening velocities, but are less fatigue-resistant than the slow twitch fibres. Fast twitch fibres can be further divided in fast-glycolytic (IIA) and fast-oxidative fibres (IIB). In between the distinct muscle fibres, a network of small blood vessels and capillaries is present for the supply of nutrients and oxygen, and for the removal of end products of muscle cell metabolism. 
The muscle cell itself is enclosed by a membrane, designated as sarcolemma. The cytoplasm (sarcoplasm) contains structures with highly specialised functions, i.e., the smooth endoplasmatic reticulum (sarcoplasmatic reticulum, SR) serves, among others, as a compartment for uptake and release of the calcium ions, which are required for muscle contraction (2). The transversal (T) tubules, being invaginations of the sarcolemma, play an important role in action potential propagation, which is the trigger for intracellular $\mathrm{Ca}^{2+}$ release from the SR (3).

Muscle fibres consist of several hundred to thousand myofibrils, together forming the contractile machinery of the skeletal muscle. The myofibrils in turn consist of long strands of smaller subunits, i.e., the sarcomeres. Sarcomeres are composed of two types of contractile protein filaments, actin and myosin (4). The regular arrangement of the filaments gives the myofibril, and the muscle fibre, the striated-banded appearance under the microscope (fig. 2.1) (5). Actin is associated with a long fibrous protein, tropomyosin, to which a complex of three subunits of troponin is attached, i.e., troponin I, C, and T. The tropomyosin extends over actin subunits and regulates binding of the myosin globular head to actin by a conformational change of the troponin complex, by elevated levels of calcium ions (6). Myosin, also referred to as thick filament, consists of two identical heavy chains, forming a $\alpha$-helix, each ending in the above mentioned myosin heads, at which the actin interaction site and ATPase activity are located (7). The thick myosin filaments are arranged so that the thin filaments can slide in between.

figure 2.1 Electron photomicrograph of a single sarcomere. Distinct regions of the sarcomere are depicted (see text for explanation).

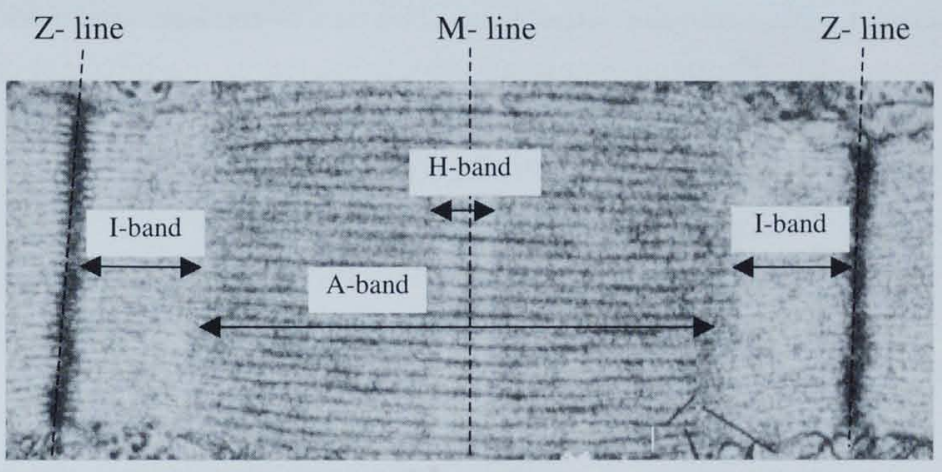

Visually the sarcomere can be separated in various distinct regions (fig. 2.1). The Aband can microscopically be distinguished as a wide, relatively dark zone, corresponding with the total length of a myosin molecule. The area in the A-band, without any overlap with the thin filaments, is known as the H-band. 
The M-line is located centrally in the H-band, and contains structural proteins for the spatial arrangement of the myosin filaments. The I-band is the region consisting of thin filaments alone. In the middle of the I-band, the Z-line or Z-disc is visible. The Z-line consists of a sheet of $\alpha$-actinin molecules, which anchors the thin filaments and connects each myofibril to adjacent myofibrils (8). The sarcomere structure is maintained by cytoskeletal proteins, keeping the contractile machinery in the proper spatial arrangement. Nebulin, which is connected to the actin filaments, is supposed to regulate the length of the actin filament (9). The Z-line and M-line distance is spanned by a titin molecule (ca. $3000 \mathrm{kDa}$ ). Titin plays an important role in maintaining the myosin alignment and the transmission of the passive force (10). Desmin is the most prominent protein present in close vicinity of the sarcomeres in the mature skeletal muscle (11). This protein connects the adjacent Z-lines in the longitudinal and transversal directions and is responsible for the $\mathrm{Z}$-line register.

\section{3 - Molecular Mechanisms of Skeletal Muscle Contraction}

In the adult skeletal muscle, one motoneuron innervates a number of fibres and this functional complex is called a motor unit. When a motoneuron fires, all fibres in that unit will contract simultaneously (12). The axon branch and the muscle fibre are connected via the neuromuscular junction, i.e., synapse. An action potential of a motoneuron will result in the release of acetylcholine (ACh) in the synaptic cleft. ACh binds to ACh receptors on the post-synaptic muscle fibre membrane, resulting in depolarisation of this membrane. The action potential propagates along the sarcolemma, and reaches the muscle interior via the $T$ tubules (12). Depolarisation of the sarcolemma and the $\mathrm{T}$ tubular membrane evokes the release of $\mathrm{Ca}^{2+}$ from the sarcoplasmatic reticulum into the sarcoplasm. The part of the sarcoplasmatic reticulum in close vicinity to the $\mathrm{T}$ tubular membrane is designated as the terminal cisternae. In this region, the bulk of intracellular $\mathrm{Ca}^{2+}$ is stored (12).

Triggered by the action potential, large amounts of $\mathrm{Ca}^{2+}$ ions are released into the sarcoplasm by the voltage-gated $\mathrm{Ca}^{2+}$ channels in the sarcoplasmatic reticulum, raising the cytoplasmic $\mathrm{Ca}^{2+}$ concentration from $10^{-7}$ to $10^{-5} \mathrm{M}$. At a critical $\mathrm{Ca}^{2+}$ threshold concentration, calcium ions combine with troponin $\mathrm{C}$. This binding results in a conformational change of the $\mathrm{T}$ and $\mathrm{I}$ subunits of the troponin complex. Consequently, the tropomyosin strands move deeper into the groove of the actin filament (13), and the myosin binding sites at the actin filaments become exposed. The globular heads of myosin will subsequently bind to the actin filament, forming the socalled cross bridges. This process of fibre activation via action potential propagation, calcium release and myosin-actin interaction is known as the excitation-contraction coupling. 
Contractile activity of muscle fibres by electrical stimulation is commonly followed by relaxation. The membrane potential of the sarcolemma returns to resting values (-60 $\mathrm{mV}$ ), as does the membrane of the T tubular and SR system (12). Subsequently, sarcoplasmatic $\mathrm{Ca}^{2+}$ is pumped back into the SR cisternae by an ATP-driven calcium pump (SERCA) (14). For each ATP hydrolysed, two $\mathrm{Ca}^{2+}$ ions are taken up by the sarcoplasmatic reticulum, where it is mostly bound to calsequestrin proteins. The drop in intracellular $\mathrm{Ca}^{2+}$ concentration causes the return of the tropomyosin molecule from the groove to the original position at the surface of the actin filament. Through this change in position, the active sites of the actin filaments are covered by tropomyosin, which hampers the formation of new cross bridges, and the muscle relaxes (15). Our current knowledge of the molecular mechanisms of muscle contraction is based on the sliding-filament model proposed by Huxley in the late fifties (16). He observed with the interference microscope that during contraction the I-band shortens while the length of the A-band remains unchanged, which could be explained by a sliding movement of the actin and myosin filaments in between each other. The general framework of the cross-bridge kinetic theory was first proposed by Lymn and Taylor (1971), who demonstrated that hydrolysis of adenosine triphosphate (ATP) is the main driving force in cross-bridge cycling (17). Before myosin heads bind to the actin filaments, myosin-bound ATP is hydrolysed by myosin ATPase into adenosine diphosphate (ADP) and inorganic phosphate $\left(\mathrm{P}_{\mathrm{i}}\right)$, i.e., myosin exists in a high-energy conformational state (fig. 2.2, state 1).

figure 2.2 Schematic representation of the cross-bridge cycle.

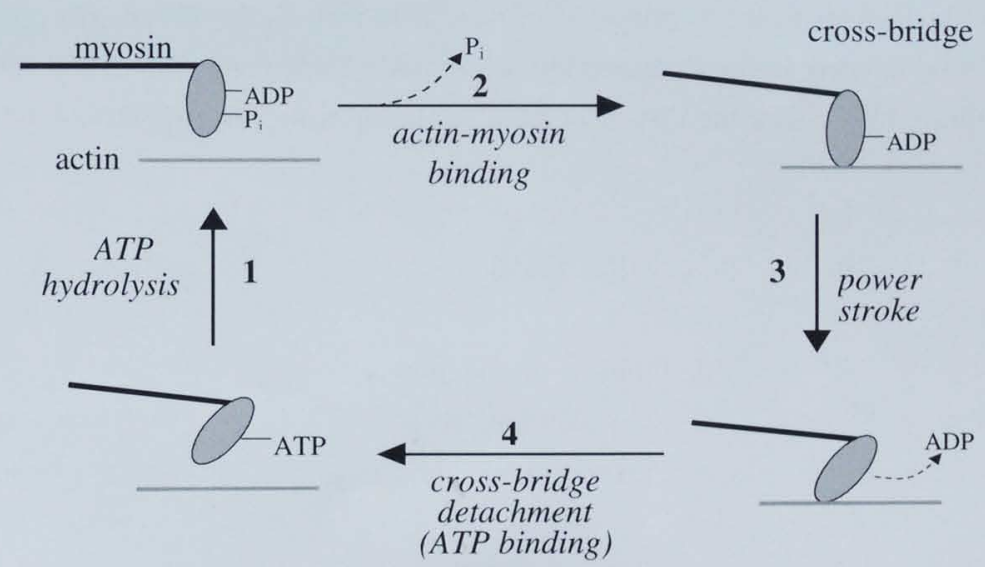

ATP, ADP, and $\mathrm{P}_{\mathrm{i}}$ refer to adenosine triphosphate, adenosine diphosphate, and inorganic phosphate, respectively (adapted from Cooke, 1997) 
Due to enhanced $\mathrm{Ca}^{2+}$ concentration, the myosin head bounds tightly to actin, while $\mathrm{P}_{\mathrm{i}}$ is released (state 2). Subsequently, the myosin head tilts backward, providing the power stroke for pulling the actin filament, resulting in the production of force (state 3). This conformational change also allows the release of ADP (low-energy conformational state) and exposes a site on the myosin-head for binding a new ATP molecule. Detachment of the globular head will occur when intracellular $\mathrm{Ca}^{2+}$ drops below threshold levels (state 4). The complete cycle of the actomyosin interaction is referred to as the cross-bridge cycle (4).

\section{4 - Functional Characteristics of Skeletal Muscle}

Electrical stimulation of the muscle will result in force production, but the degree of force produced depends, among others, on muscle intrinsic properties, sarcomere length and contractile velocity. Moreover, the nature of the contraction also plays an essential role in the degree of force production. Skeletal muscle contraction can be divided into three types of contraction, i.e., isometric, concentric and eccentric contractions. During isometric contractions, the length of the muscle complex remains constant, whereas during concentric and eccentric the length of the muscle complex respectively shortens or lengthens during stimulation of the muscle fibres.

The major aim of the present thesis was to assess contractile performance of the muscle in its natural surrounding. Consequence of studying the intact muscle complex is that it is not possible to directly determine muscle force. Otherwise, one of the tendons of the muscle of interest has to be cut and fixed to a force transducer. In our developed experimental set-up, muscular contractility is determined in terms of muscle torque. Muscular torque is the product of muscle force times the lever arm (eqn. 2.1). The lever arm is defined as the distance from the ankle joint perpendicular to the force direction generated by either the dorsal or plantar flexor complex (fig. 2.3).

$$
\mathrm{T}=\mathrm{F} \times \mathrm{d}
$$

$$
\begin{aligned}
& \mathrm{T}: \text { muscle torque, } \mathrm{mN} \cdot \mathrm{m} \\
& \mathrm{F}: \text { muscle force, } \mathrm{N} \\
& \mathrm{d}: \text { lever arm, } \mathrm{m}
\end{aligned}
$$


figure 2.3 Schematic drawing of the ankle joint and dorsal flexor and plantar flexor complexes.

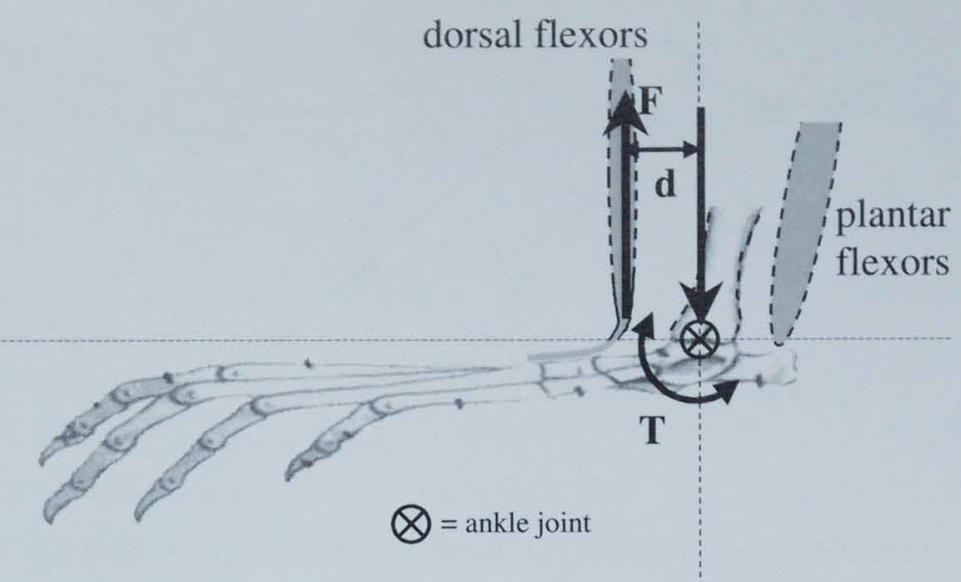

Torque $(\mathrm{T})$, force $(\mathrm{F})$, and lever arm (d) are illustrated for the dorsal flexor complex.

When discussing force development as one of the functional properties of the skeletal muscle complex, torque in stead of force can be read, assuming that the lever arm is a constant factor. The isometric force that a muscle complex generates, is primarily a function of its physiological cross-sectional area (PCSA) (eqn. 2.2).

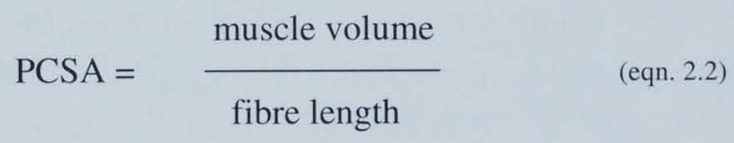

The denominator of equation 2.2 is the mean length of the muscle fibres at optimal sarcomere length. According to the sliding filament model, force generation depends on sarcomere length, and therefore on muscle complex length. The length dependence of muscle force is described by the force-length curve. In the late $19^{\text {th }}$ century, Blix was the first to construct a force-length curve from a series of isometric contractions performed at different lengths of frog muscle $(18,19)$. The main feature of the force-length relationship is that produced force depends on the degree of overlap of the myosin and actin filaments (fig. 2.4). The muscle fibres produce maximal force output at optimal sarcomere length $(\sim 2.2 \mu \mathrm{m})$, whereas the force declines on either side of the optimum length. 
figure 2.4 Force-sarcomere length relationship of a striated muscle fibre.

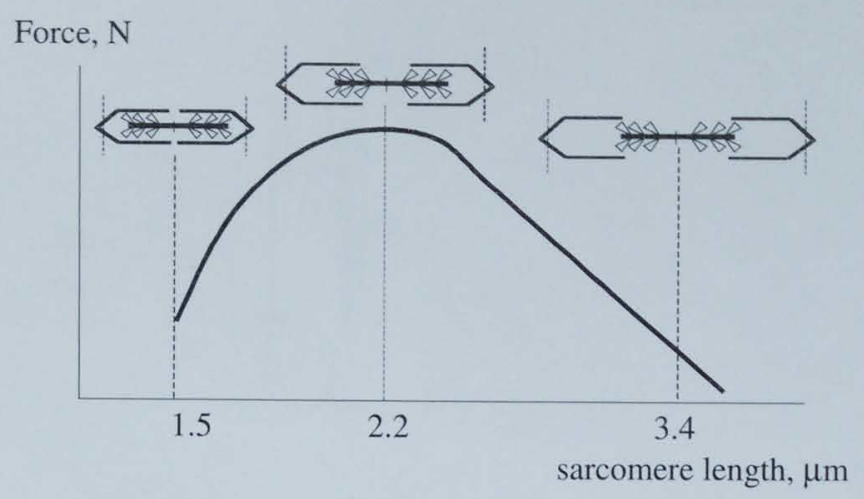

The second property of skeletal muscle is the relation between force and contraction velocity. The force-velocity relationship of a contracting muscle was first characterised by Hill (1938) and showed a lower force output at higher shortening contraction velocity (20) (see upper part of fig. 2.5). The number of cross bridges attached decreases at high shortening velocity, simply due to the fact that at high velocities little time is available for cross bridge attachment. The greater force output during an eccentric contraction compared to the concentric contraction is probably due to the compliant structure of a myosin head that is more stretched during eccentric than during concentric contractions (12). During an isometric contraction of a muscle complex (zero velocity), no external work is produced and all energy is released as heat. This is in contrast to shortening and lengthening contractions, where external work is produced in addition to heat. External work of an active muscle complex can be calculated as the integral of active force over the distance travelled. In our experimental set-up, external work is calculated as the integration of active torque over rotation angle. If we take two identical muscle complexes performing equal amounts of external work, but in different time spans, it is said that the fastest muscle is the most powerful muscle. Power is the rate of performing external work and its unit of measurement is Watt. For muscles, power is calculated as the product of active muscle force times the speed of shortening contraction (fig. 2.5). When determining the forcevelocity properties of a muscle complex, the fibre type distribution must be taken in account $(21,22)$. Slow- and fast twitch fibres with the same PCSA may have similar isometric force production, but they differ substantially in maximal and optimal shortening velocity. Therefore, the difference in fibre type composition has in particular its impact on power output. The power output of slow-twitch fibres is much lower at all speeds of shortening than that of fast-twitch fibres. 
figure 2.5 Force-velocity relationship (top panel) and power-velocity relationship (lower panel) of a striated muscle fibre (adapted from (1)).

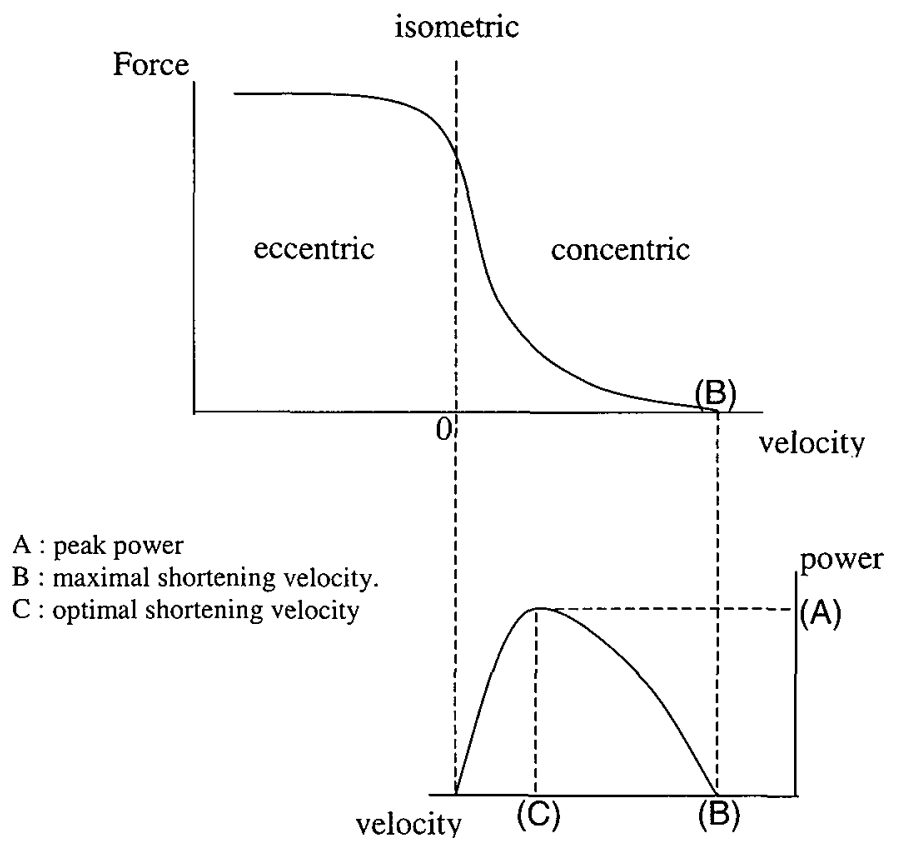

\section{5 - Energy Metabolism of Skeletal Muscle}

The universal energy donor in the skeletal muscle cell for energy utilising processes, such as ion pumps and a number of distinct steps in metabolic pathways, and the contractile machinery itself, is adenosine triphosphate (ATP) (eqn. 2.3).

$$
\mathrm{ATP} \leftrightarrow \mathrm{ADP}+\mathrm{P}_{\mathrm{i}}+\text { energy }
$$

Both the ATP concentration and the ATP to ADP ratio exert important regulatory functions in the skeletal muscle fibre (3). Figure 2.6 represents a schematic overview of the metabolic pathways for ATP production in the muscle cell. Nature has developed several energy producing systems to replenish the ATP pool in the cytoplasm of the muscle cell when energy demand is increased. An additional highenergy phosphate pool is present in the sarcoplasm in the form of phosphocreatine (PCr). The creatine kinase $(\mathrm{CK})$ isoenzymes catalyse the transphosphorylation reaction between PCr and ATP (23). Five CK isoenzymes are currently known in mammalian tissues, from which three of them are located within the cytoplasm and two in the mitochondria. 
The cytosolic $\mathrm{CK}$ isoforms form dimeric molecules composed of the muscular subunits (M) and the brain type subunits (B), i.e., MM-CK, MB-CK, and BB-CK (23). The two mitochondrial $\mathrm{CK}$ isoenzymes are called either ubiquitous or sarcomeric, $\mathrm{M}_{\mathrm{i}}$ $\mathrm{CK}$, after their tissue-specific expression (23). Skeletal muscle contains the MM-CK and sarcomeric $\mathrm{M}_{\mathrm{i}}$-CK isoforms of creatine kinase $(24,25)$. The primary function of the CK system is that of temporal energy buffering, but the occurrence of several isoenzymes indicates a broader function of the CK system. A second main function in spatial buffering of energy has been assigned to the CK system, i.e., $\mathrm{PCr}$-shuttle (26). In this model, the high-energy phosphate of ATP, synthesised in the mitochondrion, is transferred to $\mathrm{PCr}$ via $\mathrm{M}_{\mathrm{i}}-\mathrm{CK}$. PCr diffuses towards sites in the cell where energy is consumed; the high-energy phosphate of PCr is transphosphorylated to generate ATP catalysed by MM-CK (26).

figure 2.6 Schematic overview of the metabolic pathways for ATP production in the muscle cell.

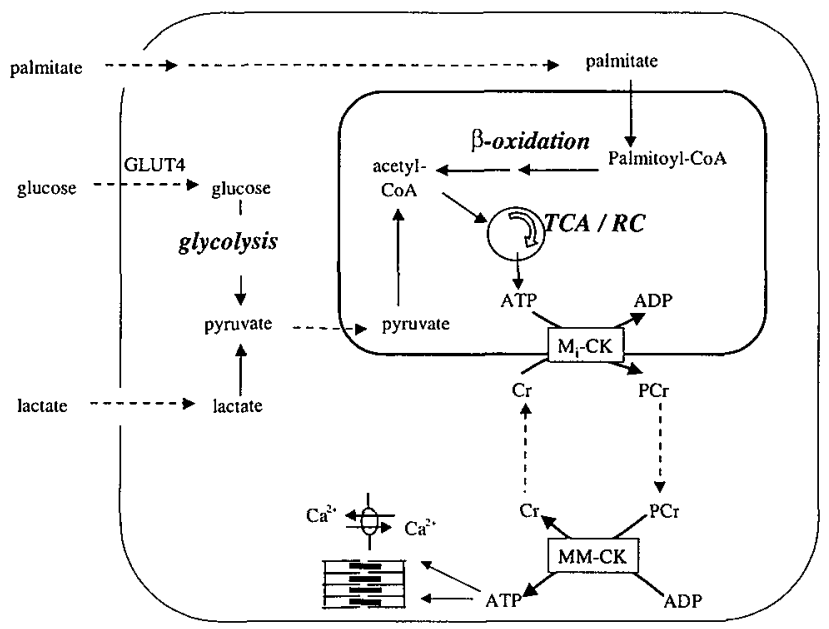

Detailed explanation is given in the text. Dashed and solid lines represent transport mechanism and metabolic reactions, respectively. TCA and RC represent the tricarboxylic acid cycle and the respiratory chain, respectively. The mitochondrial isoform of creatine kinase is denoted as $\mathrm{M}_{\mathrm{i}}-\mathrm{CK}$ and the cytosolic isoform as MM-CK.

A second ATP regenerating process is the glycolytic conversion of glucose into pyruvate. Two pathways are involved in the metabolism of glucose, i.e., anaerobic and aerobic. The anaerobic process occurs in the cytoplasm involving the conversion of pyruvate to lactate, and produces a moderate amount of ATP. The aerobic combustion of pyruvate-derived carbon atoms takes place in the mitochondria and results in a substantial amount of ATP, and as the name implies, requires molecular oxygen. 
Since the lipid bilayer of the sarcolemma represents a barrier for hydrophilic substances, glucose is taken up via carrier-mediated facilitated diffusion rather than simple diffusion. To date, a family of eight glucose transporters (GLUT) have been identified. GLUT1, which is involved in basal glucose transport, and GLUT4, which is instrumental in insulin and contraction stimulated glucose transport, are both present in the skeletal muscle cells (27). Glucose transport in these cells is a unidirectional process, because intracellularly glucose is trapped by phosphorylation to glucose-6phosphate. Muscle cells, in contrast to hepatocytes, virtually lack the enzyme glucose 6-phosphatase, which dephosphorylates glucose-6-phosphate to glucose (28). In the first stage of the glycolytic pathway, the glucose part of glucose 6-phosphate is rearranged to fructose and phosphorylated further to fructose diphosphate. The first steps of the glycolytic pathway actually require energy, in the form of two ATP molecules per glucose. Fructose diphosphate is then cleaved to yield two glyceraldehyde phosphates (GPs). In the next steps, energy is finally released, in the form of two ATP and two NADH molecules, when the GPs are oxidised to phosphoglycerates. Finally, two more ATP molecules are produced when the phosphoglycerates are oxidised to pyruvate. As discussed above, pyruvate can be converted into lactate under anaerobic circumstances or is the starting molecule for oxidative phosphorylation via the citric acid cycle. In the latter case, the pyruvate is converted inside the mitochondrion into acetyl coenzyme A, which subsequently binds with the four-carbon oxaloacetate to generate the six-carbon citrate. Carbons and hydrogens are gradually cleaved from citrate until the four-carbon oxaloacetate is recycled. In this process, four $\mathrm{NADH}$, one $\mathrm{FADH}_{2}$ and one GTP are generated for each starting pyruvate. Each $\mathrm{NADH}$ will be oxidised to $\mathrm{NAD}^{+}$, generating three ATP molecules via oxidative phosphorylation, $\mathrm{FADH}_{2}$ yields two ATP, and GTP generates one ATP molecule. Thus, for each glucose that is taken up by the muscle, up to 36 ATP molecules can be aerobically generated (28).

Blood borne fatty acids are also an important metabolic fuel for the replenishment of ATP in skeletal muscle. The first step in the uptake of fatty acids is their translocation through the endothelial cells. Next, the fatty acids have to be transported through the interstitial space, most likely bound to albumin (29). The sarcolemma is passed either via passive diffusion or via facilitated diffusion by plasma membrane transporters, such as $\mathrm{FABP}_{\mathrm{pm}}$ and FAT (30). Unlike glucose, fatty acids are poorly soluble in water, and, hence, transport of fatty acids from the sarcolemma to the mitochondria is facilitated by cytoplasmic fatty acid-binding proteins $\left(\mathrm{FABP}_{\mathrm{c}}\right)$ (31). Subsequently, the bulk of the fatty acids are utilised for oxidative energy conversion in the mitochondria to produce ATP. Transport of the fatty acyl moieties into the mitochondria is mediated by carnitine. 
Inside the mitochondria, fatty acyl-CoA is degraded to acetyl coenzyme-A via the $\beta$ oxidation (28). After condensation with oxaloacetate to citrate, the carbon atoms and hydrogen atoms of acetyl coenzyme- $\mathrm{A}$ are oxidised to $\mathrm{CO}_{2}$ via the tricarboxylic acid cycle and respiratory chain activity, which results in a relatively large amount of ATP. For instance, the oxidation of one mole palmitic acid yields 129 moles of ATP (32).

\section{6 - Energy metabolism during muscular contractions}

Carbohydrates and fatty acids are considered the most prominent substrates during muscular contractions (33). In general, more than $50 \%$ of the energy of resting muscular tissue is derived from fatty acids oxidation (32). Acute, high-intensity muscular activity, such as weight lifting or sprinting, relies on the cellular high-energy phosphates ATP and PCr. Furthermore, anaerobic metabolism of glycogen and blood borne glucose contribute in the energy supply during these types of muscular performance. The aerobic pathways of producing energy are less suitable since limited amounts of oxygen are available. Moreover, these aerobic pathways for ATP generation are too slow to match fully the rate of ATP utilisation (34). During exercise of moderate intensity, carbohydrates become important substrates for the muscle cells, but the relative contribution declines with increasing contraction duration and decreasing contraction intensity (31). This is in contrast to the relative contribution of fatty acids, which becomes more important with increasing duration of the contraction series (33). 


\section{7 - References}

1. Epstein, M., and Herzog, W. (1998) Theoretical models of skeletal muscle., John Wiley \& Sons, Chichester

2. Peachey, L. D. (1965) The sarcoplasmic reticulum and transverse tubules of the frog's sartorius. J. Cell. Biol. 25, Suppl:209-231

3. McComas, A. J. (1996) Skeletal muscle, form and function, Human Kinetics, Leeds

4. Cooke, R. (1997) Actomyosin interaction in striated muscle. Physiol. Rev. 77, 671-697

5. Cohen, C., and Vibert, P. J. (1987) Fibrous protein structure, Academic Press, London

6. Patel, T. J., Richard, M. S., and Lieber, L. (1997) Force Transmission in skeletal muscle: from actomyosin to external tendons. Ex. Sports Scienc. Rev. ?, 321-363

7. Knight, P., and Trinick, J. (1987) Fibrous protein structure, Academic Press, London

8. Franzini-Armstrong, C. (1973) The structure of a simple Z line. J Cell Biol 58, 630642

9. Labeit, S., and Kolmerer, B. (1995) The complete primary structure of human nebulin and its correlation to muscle structure. J. Mol. Biol. 248, 308-315

10. Wang, K., McCarter, R., Wright, J., Beverly, J., and Ramirez-Mitchell, R. (1993) Viscoelasticity of the sarcomere matrix of skeletal muscles. The titin-myosin composite filament is a dual-stage molecular spring. Biophys. J. 64, 1161-1177

11. Lazarides, E. (1980) Desmin and intermediate filaments in muscle cells. Cell Diff. 11, 124-131

12. Jones, D. A., and Round, J. M. (1993) Skeletal muscle in health and disease, a textbook of muscle physiology., Manchester University Press, Manchester

13. Zot, A. S., and Potter, J. D. (1987) Structural aspects of troponin-tropomyosin regulation of skeletal muscle contraction. Ann. Rev. Biophys. Chem. 16, 535-559

14. Pozzan, T., Rizzuto, R., Volpe, P., and Meldolesi, J. (1994) Molecular and cellular physiology of intracellular calcium stores. Physiol. Rev. 74, 595-636

15. Westerblad, H., and Allen, D. G. (1994) The role of SR in relaxation of mouse muscle. J. Physiol. 474, 291-301

16. Huxley, A. F. (1957) Muscle structure and theories of contraction. Biophys. biophysic. chem. 7, 255-318

17. Lymn, R. W., and Taylor, E. W. (1971) Mechanism of adenosine triphosphate hydrolysis by actomyosin. Biochem. 10, 4617-4624

18. Blix, M. (1893) Die lange und spannung des muskles. Skand. Arch. Physiol. III, 295318

19. Blix, M. (1893) Die lange und spannung des muskles, zweite abhandlung. Skand. Arch. Physiol. IV, 400-409

20. Hill, A. V. (1938) The heat of shortening and the dynamic constants of muscle. Proc. Roy. Soc. London B. 126, 136-195

21. Brooks, S. V., and Faulkner, J. A. (1991) Forces and powers of slow and fast skeletal muscles in mice during repeated contractions. J. Physiol. 436, 701-710

22. Widrick, J. J., Trappe, S. W., Costill, D. L., and Fitts, R. H. (1996) Force-velocity and force-power properties of single muscle fibers from elite master runners and sedentary men. Am. J. Physiol. 271, C676-683

23. Wyss, M., Smeitink, J., Wevers, R. A., and Walliman, T. (1992) Mitochondrial creatine kinase: a key enzyme of aerobic energy metabolism. Biochim. Biophys. Acta $1102,119-166$ 
24. Schlegel, J., Zurbriggen, B., Wegmann, G., Wyss, M., Eppenberger, H. M., and Wallimann, T. (1988) Native mitochondrial creatine kinase forms octameric structures. I. Isolation of two interconvertible mitochondrial creatine kinase forms, dimeric and octameric mitochondrial creatine kinase: characterization, localization, and structure-function relationships. J. Biol. Chem. 263, 16942-16953

25. Walliman, T., Wyss, M., Brdiczka, D., and Nicolay, K. (1992) Intracellular compartmentation, structure and function of creatine kinase isoenzymes in tissues with high and fluctuating energy demands: the 'phosphocreatine circuit' for cellular energy homeostasis. Biochem. J. 281, 21-40

26. Bessman, S. P., and Carpenter, C. L. (1985) The creatine-creatine phosphate energy shuttle. Annu. Rev. Biochem. 54, 831-862

27. Bell, G. I., Kayano, T. K., Buse, J. B., Burant, C. F., Takeda, J., Lin, D., Fukumoto, H., and Seino, S. (1990) Molecular biology of mammalian glucose transporters. Diab. Care 13, 198-208

28. Newsholme, E. A., and Leech, A. R. (1994) Biochemistry for the medical sciences, John Wiley \& Sons, New York

29. Van der Vusse, G. J., and Reneman, R. S. (1995) Substrate utilization in mammalian cells. Princ. Med. Biol. 4, 45-75

30. Luiken, J. J., Schaap, F. G., van Nieuwenhoven, F. A., van der Vusse, G. J., Bonen, A., and Glatz, J. F. (1999) Cellular fatty acid transport in heart and skeletal muscle as facilitated by proteins. Lipids 34, S169-175

31. Van der Vusse, G. J., and Reneman, R. S. (1996) Lipid metabolism in Muscle. In Exercise: regulation and integration of multiple systems. (Rowell, L. B., and Sheperd, J. T., eds) pp. 953-994, Oxford University Press, Oxford

32. Gollnick, P. D., and Saltin, B. (1988) Fuel for muscular exercise: role of fat. In Exercise, nutrition, and energy metabolism, pp. 71-88.

33. Romijn, J. A., Coyle, E. F., Sidossis, S., Gastaldelli, A., Horowitz, J. F., Endert, E., and Wolfe, R. R. (1993) Regulation of endogenous fat and carbohydrate metabolism in relation to exercise intensity and duration. Am. J. Physiol. 265, E380-E391

34. Van der Vusse, G. J., and de Groot, M. J. (1992) Interrelationship between lactate and cardiac fatty acid metabolism. Mol Cell Biochem 116, 11-17 


\section{Chapter 3}

\section{Accurate assessment of in situ isometric CONTRACTILE PROPERTIES OF HINDLIMB PLAN- TAR AND DORSAL FLEXOR MUSCLE COMPLEX OF INTACT MICE.}

M. Gorselink, M.R. Drost, J. de Louw, P.J.B. Willems, N. Rosielle, J.D. Janssen, G.J. van der Vusse, (2000) Eur. J. Physiol.,Instruments and Techniques 439: 665-670.

Description of the isometric dynamometer for assessment of isometric contractile properties of intact dorsal and plantar flexors of the mouse. 


\section{1 - Summary}

An isometric torque sensor for in situ measurements of plantar or dorsal flexors properties of intact mouse hind limb has been developed and evaluated. With this device muscle torque during isometric contractions could be accurately measured within the range of $-14 \mathrm{mN} \cdot \mathrm{m}$ and $+14 \mathrm{mN} \cdot \mathrm{m}$. Special attention was paid to the fixation procedure of the mouse to the measurement device. Halothane- anaesthetised Swiss wild-type mice were positioned on the thermostatic measurement platform, and fixated with a hip and foot fixation unit. Plantar or dorsal muscle complexes were electrically stimulated. The novel fixation unit was evaluated by measuring knee and ankle displacements during contractions. The functional consequences of these hind limb movements were evaluated with a mathematical muscle model.

Measured ankle and knee displacement resulted in a calculated muscle fibre shortening of $2.5 \%$. Simulations of a contraction with this fibre shortening, using the mathematical muscle model, showed only minor effects on maximal torque and the temporal parameters. Furthermore, we showed that muscle torque in our set-up is hardly affected by misalignment between the ankle and measurement axis, i.e., eccentricity. Measured tetanic muscle torques of intact dorsal and plantar flexors were $3.2 \pm 0.4 \mathrm{mN} \cdot \mathrm{m}$ and $11.8 \pm 1.6 \mathrm{mN} \cdot \mathrm{m}$, respectively. The $1 / 2$-relaxation time of plantar flexors was significantly higher than in dorsal flexors $(14.9 \pm 1.6 \mathrm{~ms}$ vs. $11.8 \pm 2.0$ $\mathrm{ms})$, whereas the $10-50 \%$ rise time was longer in plantar $(13.4 \pm 1.2 \mathrm{~ms})$ than in dorsal (10.4 $\pm 1.1 \mathrm{~ms})$ flexors. 


\section{2 - Introduction}

Recently, the generation of transgenic mice has greatly improved the possibilities for studying the specific role of proteins and enzymes in muscle energy metabolism and mechanical functioning. Muscle contractile activity in small rodents, such as mice, is mostly studied with the use of invasive techniques $(3,4,5)$. Since we wanted to investigate muscle mechanical properties under conditions as physiologically as possible, we developed an in situ experimental model for exploring isometric contraction characteristics of dorsal and plantar muscles, leaving the muscle complex in its natural environment. It should be emphasised that in this experimental set-up, isometric contraction comprise the total muscle complex. After the onset of contraction, tendons and tendon sheets elongate allowing the muscle fibres to shorten (10).

With respect to the analysis of muscular mechanical activity in the intact mouse, pioneering experiments were performed by Ashton-Miller et al. (1). They studied the mechanical behaviour of murine dorsal and plantar flexor muscles in situ. Taking their experimental approach as a starting point, we designed an experimental device to determine in situ isometric muscle torque and temporal contraction characteristics of plantar and dorsal flexors around the mouse ankle joint. For accurate measurements of muscle functioning in small animals, like mice, the following criteria are essential. First, resonance frequency of the measurement system must be higher than the fastest relevant frequency component of the measured torque. Second, stiff fixation of the animal to the measuring device is necessary to prevent displacement of bones. Any movements of bones due to loose fixation causes shortening of the muscletendon complex and, hence, influence the force generation. Furthermore, the ankle axis should be aligned to the apparatus shaft as good as possible, although some degree of eccentricity between the two axes cannot be prevented. In commonly used rigid knee fixation methods, this eccentricity will affect the measured muscle torque, because an additional fixation force will result in a non-muscle torque with a magnitude of the fixation force times the eccentricity distance (1). Therefore, a new hip fixation unit and an improved foot fixation system have been developed in order to minimise ankle and knee displacements, and hence, to prevent the unfavourable fixation forces.

This paper provides a detailed description of the isometric torque measurement unit and the fixation procedure. Knee and ankle displacements during a maximal contraction were monitored. A recently described mathematical muscle model $(13,14)$ was used to quantify the effects of non-absolute fixation on the contractile parameters. Furthermore, some selected mechanical parameters of dorsal and plantar flexors of intact mice are presented. 


\section{3 - Materials and methods}

\subsubsection{Experimental procedure}

All experiments $(n=6)$ were performed on 16 week old male Swiss mice $(28.0$ $\pm 2.1 \mathrm{~g}$; mean \pm standard deviation), positioned on an aluminium platform maintained at $38.5 \pm 0.1^{\circ} \mathrm{C}$. Mice were anaesthetised with $1.5-2.0 \%$ Halothane (Fluothane ${ }^{\circledR}$, Zeneca, Ridderkerk, The Netherlands) which was mixed with $\mathrm{O}_{2}$ and $\mathrm{N}_{2} \mathrm{O}(2: 1,3.0$ $\left.1 \cdot \mathrm{min}^{-1}\right)$, and subsequently delivered via a facemask through a flowmeter system (Medec, Montvalle, N.J., USA). All experimental procedures were approved by the Institutional Animal Care and Use Committee of the Maastricht University and complied with the principles of proper laboratory animal care.

In general, an anaesthetised mouse was fixated via the hip to a thermostatic platform and the foot was rigidly connected to the measurement device. The plantar or dorsal muscle complexes were electrically stimulated via their respective nerves. Muscle torque around the ankle joint was measured using a custom-built torque sensor. Figure 3.1 shows a schematic outline of the experimental set-up and the principle of muscle torque determination around the ankle joint.

\subsubsection{Apparatus description}

During the experiment, the mouse foot was rigidly connected to the shaft of the torque transducer (see below). This shaft is connected to two perpendicular positioned torsion springs ( $8 \mathrm{~mm} \times 7 \mathrm{~mm} \times 0.08 \mathrm{~mm}$ ). During contraction, produced plantar or dorsal muscle torque results in a minor rotation $\left(<0.2^{\circ}\right)$ of the shaft, causing a slight deformation of the torsion springs. The deformation of the torsion springs is monitored by magnetic induction with two micro-sensors (HA-30S, micro-epsilon Messtechnik GmbH\&Co, Ortenburg-Dorfbach, Germany). Based on the dimensions of the torsion springs, it was calculated that torques ranging from $-20 \mathrm{mN} \cdot \mathrm{m}$ to $+20 \mathrm{mN} \cdot \mathrm{m}$ could be measured. The lowest resonance frequency of the measurement device is determined at $\sim 210 \mathrm{~Hz}$. In between two measurements, axis angle can be varied with a servomotor (2 Watt Maxon DC motor, Interelectric, Sachseln, Switzerland) and the angle is monitored with a Hewlett-Packard encoder (HEDS 9000, accuracy: $0.04{ }^{\circ}$ ). Data acquisition is performed at $1000 \mathrm{~Hz}$ with an Apple Macintosh 7100 PowerPC $^{\circledR}$ with an 8 channel, 12 bits Lab-NB analogue-to-digital conversion board (National Instruments, Mopac Expwy, Austin, USA) programmed with Labview 3.1 ${ }^{\circledR}$. 
figure 3.1a Schematic outline of the experimental set-up for measuring isometric torque of intact plantar and dorsal muscle complex in mice.

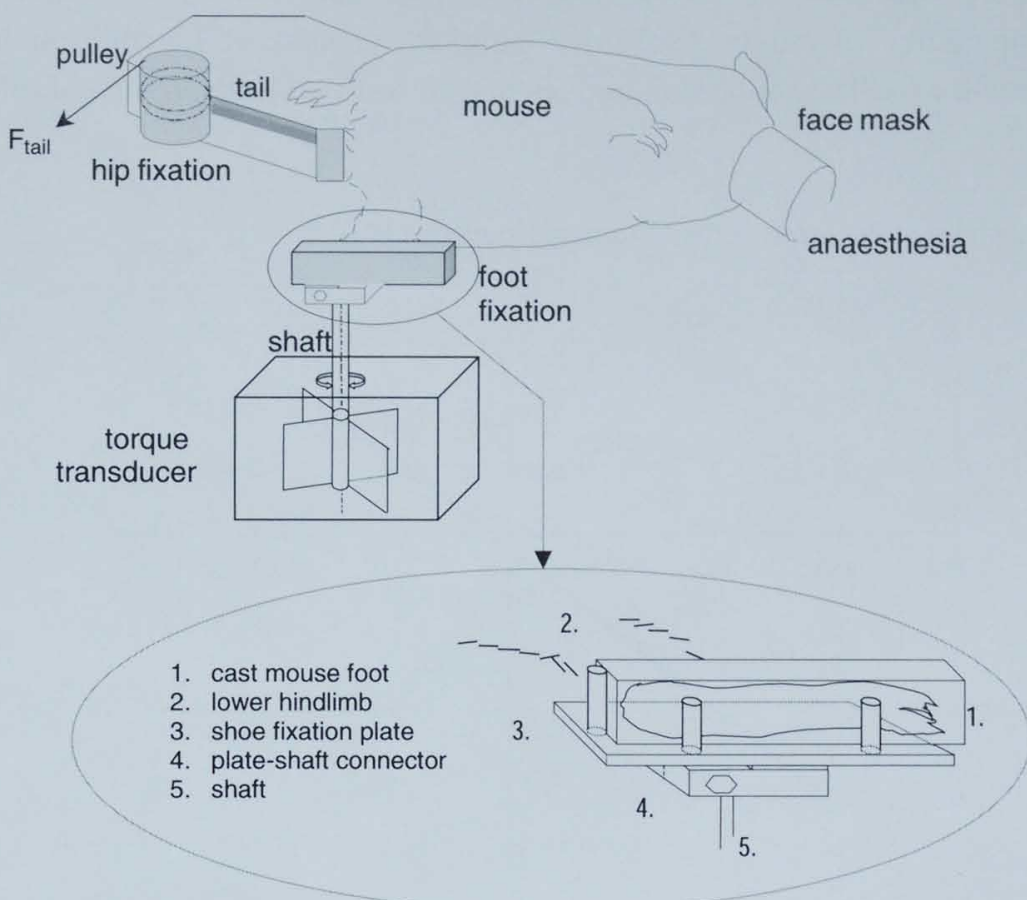

The mouse is fixated to the torque transducer shaft with a foot fixation unit, and to the external world with a hip fixation unit. Produced muscle torque is monitored with a custom-built torque transducer. A schematic outline of the foot fixation system is given in the enlargement in the lower part of the figure.

figure 3.1b Outline of the principle of measuring muscle torque around the ankle joint.

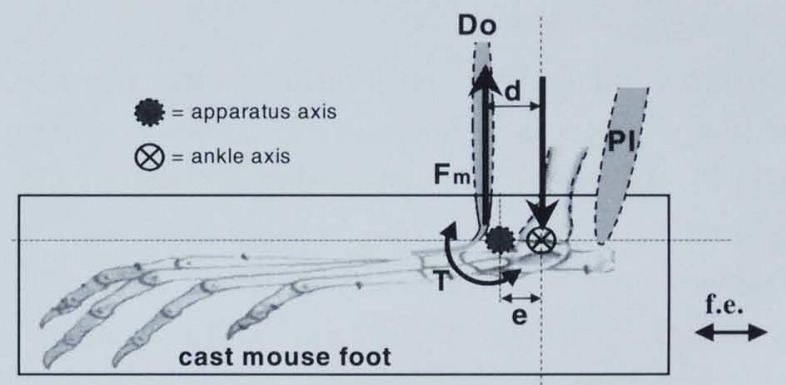

The free body diagram of the ankle joint is indicated with solid lines. Do: dorsal flexors (shown activated), PI: plantar flexors (shown not activated), $\mathrm{T}$ : torque around the ankle joint, $\mathrm{F}_{\mathrm{m}}$ : muscle force (dorsal flexors), d: lever arm of dorsal flexor complex, e: eccentricity distance between the apparatus and ankle axis, which is largely exaggerated for the sake of clarity, f.e.: freedom of variation of the cast mouse foot during the eccentricity experiments. 
The measurement unit was calibrated with an external torque calibration unit connected to the thermostatic platform. The mean calibration curve $(n=3)$ was acquired through a series of calibration torques, imposed by an increasing and decreasing number of weights $(867 \mathrm{mg} \pm 7 \mathrm{mg}$ ) at a distance of $7.5 \mathrm{~cm}$ from the torque measurement axis. The calibration torques ranged from $-14 \mathrm{mN} \cdot \mathrm{m}$ to $+14 \mathrm{mN} \cdot \mathrm{m}$ (fig. $3.2)$.

figure 3.2 Calibration curve of the torque transducer.

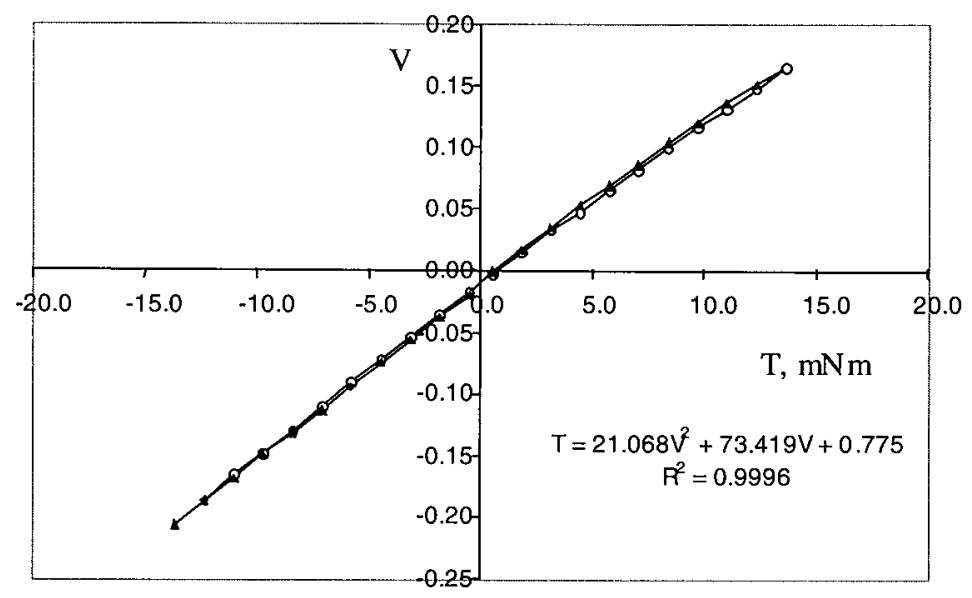

Torque transducer output is presented as voltage (V). Calibration was performed in the range of -14 to +14 $\mathrm{mN} \cdot \mathrm{m}$, using a number of weights $(867 \pm 7 \mathrm{mg})$ at a fixed distance of $7.5 \mathrm{~cm}$ from the torque unit axis. The ascending (adding weights) and descending (removing weights) limbs of the calibration curve are indicated as open circles and closed triangles, respectively.

\subsubsection{Fixation of the mouse}

An anaesthetised mouse was positioned on the thermostatic measurement platform and the foot was cast in a shoe made of a two-component cement (paladur, Heraus Kulzer GmbH, Wehrheim, Germany) using a Teflon mould. Total mass of the cement shoe $(150 \mathrm{mg})$ was relatively small compared to the mass of the mouse foot ( $300 \mathrm{mg}$ ). The shoe was glued with cyano-acrylate (Bison, Goes, The Netherlands) to a shoe-fixation plate. The connection of this plate to the apparatus shaft allowed fast removal of the hind limb from the apparatus, e.g., to freeze the muscle complex within a few seconds for biochemical analyses.

The proximal part of the hind limb was fixated to the external world through a hip fixation unit (fig. 3.1a). The mouse was positioned sidelong to the hip fixation unit and the tail was conducted through a narrow opening in the fixation platform. 
Part of the hip, tuber ischiadicum, was pulled against a vertical fixation platform by winding the tail on a pulley $\left(F_{\text {tail }}=0.5 \mathrm{~N}\right)$. With this technique, displacement of the pelvis was minimised. The knee was supported by a small Teflon plate, to prevent knee abduction while sagittal plane movements were possible. Prevention of sagittal knee movements by rigid knee fixation was not allowed, because in this case an additional fixation force would be transmitted via the tibia, introducing a non-muscular torque to the torque transducer (see below 3.3.5 and 3.3.6).

\subsubsection{Electrode placement}

Plantar or dorsal muscle complexes were stimulated via the tibial or peroneal nerve, respectively. After depilating the skin, a small incision in the hollow of the knee or in the lateral part of the knee was made to obtain access to the tibial or peroneal nerve, respectively. A bipolar platinum hook electrode, interpole distance $0.8 \mathrm{~mm}$, was carefully attached to the nerve and the muscle complex was stimulated using a pulse generator (HSE 215/IZ, Freiburg, Germany). The electrode was removed and replaced in a more distal position, if a current of more than $1.0 \mathrm{~mA}$ was required to gain fully recruited muscle contraction. The electrode was fixated to the skin with cyano-acrylate glue to prevent electrode displacement during muscle contraction.

\subsubsection{Experimental procedure to validate the fixation system}

Displacements of ankle and knee were measured for experimental validation of the fixation system. The magnitude of muscle shortening, due to these hind limb displacements, was calculated and the effect of shortening on torque generation was estimated using a mathematical muscle model $(13,14)$.

In order to monitor hind limb displacement, the malleolus medialis (ankle) and the condylus medialis (femur) were surgically exposed. Small fluorescent polystyrene spheres $(\varnothing=0.4 \mathrm{~mm})$ (Bang Laboratories, Inc. Fisshers, Carmel, Indiana, USA) were attached with cyano-acrylate glue to these bone regions. Two spheres were attached to the cast mouse foot, and two extra spheres were connected to the thermostatic measurement platform and used as fixed spheres. Plantar muscle complex was maximally stimulated for $200 \mathrm{~ms}$. Two synchronised $25 \mathrm{~Hz}$ CCD cameras (MX5, Adimec, Amsterdam, The Netherlands) with a 75-mm lens (Ernitec F1.3 TV) were positioned above the contracting muscle. Illumination intensity was manually adjusted to optimise marker contrast. Video images $(25 \mathrm{~Hz}, 400 \times 512$ pixels, shutter time: 3.2 ms) were real time digitised and stored on a Eisa 486-66 computer via the $\mathrm{R}$ and $\mathrm{G}$ input of an 8 bit RGB frame-grabber (Matrox magic, Matrox, Buckinghamshire, UK), programmed with DMAmagic (DIFA measuring systems, Breda, The Netherlands). 
The geometrical centroids of these spheres were determined from digitised image frames using TIMwin 1.3 (TEA, Dordrecht, The Netherlands). Post-processing of the centroids of marker tracks were performed with Matlab 5.2.1 (The Math Works, Inc., Natick, Massachusetts, USA). Marker displacements were calculated relative to centroids of fixed spheres during maximal plantar muscle contraction (16). Changes in the distance between the markers on the ankle and the femur are taken as the change in muscle complex length. These changes in muscle complex length can be translated to equal changes in fibre length, assuming that the tendon length only depends on muscle force, and, therefore, additional muscle shortening only occurs in the fibres. Force generation of a muscle not only depends on the fibre length (9), but also on shortening velocity via the force-velocity relationship $(2,11,12,17)$. Therefore, the fibre shortening velocity due to non-absolute fixation was calculated.

As indicated above, a mathematical muscle model of a whole muscle complex was applied, and the details are described by Maenhout and colleagues $(13,14)$. Briefly, the model consists of a contractile element (muscle fibres) in series with a non-linear elastic spring element (tendons and tendon sheets). The contractile properties were described by a two-state Huxley model $(18,19)$. A detailed description of the used model is given in Maenhout et al. $(13,14)$. Model parameters were based on values published in literature (13). The attachment rate parameter, $f_{1}$, and the detachment rate parameter, $\mathrm{g}_{1}$, were fitted manually to the torque curves, measured with the present experimental set-up. The fitted value for the attachment rate parameter, $\mathrm{f}_{1}$, was $190\left[\mathrm{~s}^{-1}\right]$ and the value for detachment rate parameter, $\mathrm{g}_{1}$, was estimated at $90\left[\mathrm{~s}^{-1}\right]$ (fig. 3.3). These values showed good agreement with previously published parameter values $(13,18,19)$.

Simulations have been made with and without changes in muscle complex length. This way, the effect of hind limb displacement, due to the non-absolute fixation, on measured parameters could be quantified. The measured shortening of the muscle-tendon complex after onset of contraction and measured lengthening during relaxation had been prescribed to the mathematical model. The amplitude of the simulated shortening was equal to the measured amplitude, and the simulated shortening velocity was chosen to be sinusoidal. 
figure 3.3 Measured normalised tetanic contraction (solid line) of a plantar flexor complex of a Swiss mouse and the corresponding simulation using a mathematical model of the muscle complex (solid line with the closed squares).

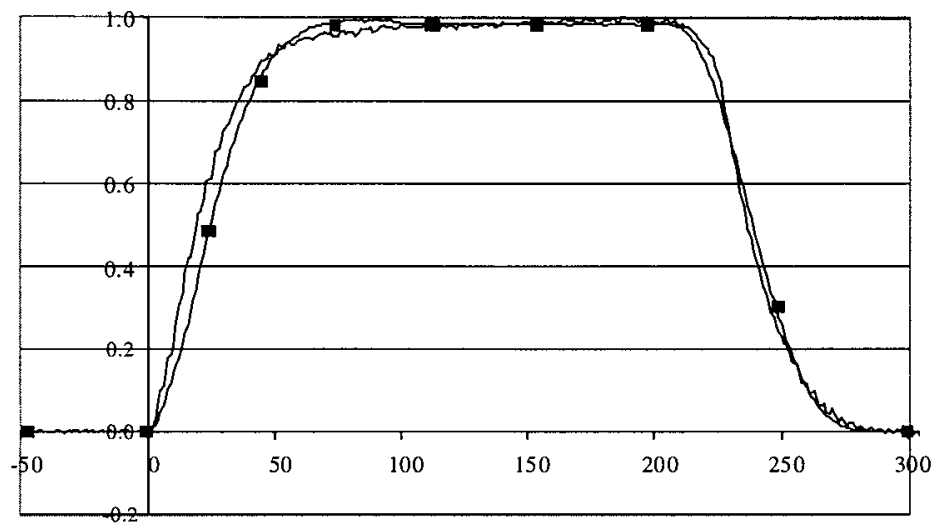

time, ms

\subsubsection{Effect of eccentricity on muscle torque}

Eccentricity is defined as the distance between the apparatus axis and the ankle axis. The eccentricity in the length direction of the mouse foot (longitudinal axis) was manipulated with a specially constructed shoe plate, allowing a manual longitudinal displacement of the mouse foot (fig. 3.1b). Two fluorescent polystyrene spheres $(\varnothing=0.4 \mathrm{~mm}$ ) (Bang Laboratories, Inc.) were attached to the cast mouse foot and exact longitudinal position was monitored with the 2-D marker tracking system (16). Visual alignment between ankle and apparatus axis was used as offset estimation. The effect of eccentricity on measured muscle torque was determined with this shoe plate by randomly varying the eccentricity between $2.0 \mathrm{~mm}$ in the toe direction and $1.5 \mathrm{~mm}$ in the heel direction.

\subsubsection{Determination of contractile muscle parameters}

Supra-maximal stimulation current is necessary to obtain full recruitment of muscle fibres and was determined using 3 to 5 twitch contractions with increasing current. Optimal ankle angle, i.e., ankle angle at maximal torque, was determined using 11 twitch contractions at ankle angles ranging from $25^{\circ}$ dorsal flexion to $25^{\circ}$ plantar flexion. Twitch contractions were used to minimise metabolic load during determination of the stimulation current for full recruitment of muscle fibres. Recovery period between twitches was $60 \mathrm{~s}$.

Maximal tetanic contraction was obtained with a $200 \mathrm{~ms}$ pulse train and a 100 $\mathrm{Hz}$ (plantar flexors) or $125 \mathrm{~Hz}$ (dorsal flexors) stimulation frequency. Twitch and tetanic torque signals were digitised $(1000 \mathrm{~Hz})$ and analysed for maximal torque value, $10-50 \%$ rise time and $1 / 2$-relaxation time (table 3.1). 
Maximal torque is the mean of the plateau of the torque signals. Ten-50\% rise time is defined as the time interval required for the muscle torque to increase from 10 to $50 \%$ of the maximal torque. The $1 / 2$-relaxation time is defined as the time interval to decrease the torque from 50 to $25 \%$ of the maximal torque (8).

\section{4 - Results and discussion}

\subsubsection{Calibration of the measurement apparatus}

The calibration curve exhibited a slight hysteresis. To explore the effect of this hysteresis, maximal torque, $10-50 \%$ rise time, and $1 / 2$-relaxation time values were calculated using either the ascending or the descending part of the calibration curve (fig. 3.2). Torque values were slightly affected $(0.9 \%)$, whereas temporal parameters were affected less than $0.01 \%$. Since hysteresis had limited effects on the calculated parameters, one polynomial curve was fit combining both the ascending and descending part of the calibration curve. The calibration curve showed a second order polynomial fit with a correlation coefficient of 0.9996 (fig. 3.2). Standard deviations of the calibration curve were maximally $0.012 \mathrm{~V}$.

\subsubsection{Resonance frequency}

The lowest resonance frequency of the measurement device was determined at $210 \mathrm{~Hz}$. A fast Fourier transform of a twitch contraction showed that $99 \%$ of the power was below $37 \mathrm{~Hz}$. This indicates that the resonance frequency of the measurement device is sufficiently high and will not interfere with the assessment of temporal contractile parameters.

\subsubsection{Validation of fixation system}

During a maximal plantar muscle contraction, the cast mouse foot showed an angular change in ankle angle of less than $0.2^{\circ}$. The femoral condyl and ankle showed a maximal displacement of $0.37 \mathrm{~mm}$ and $0.01 \mathrm{~mm}$, respectively. This hind limb displacement is caused by a $2.5^{\circ}$ knee abduction, which is a result of compression of soft tissue around the knee joint. These measured displacements resulted in a calculated maximal shortening of the distance between the ankle and femur markers of $0.17 \mathrm{~mm}$. As mentioned before, these changes in muscle complex length can be translated to equal changes in fibre length. Mean plantar fibre length in Swiss mice has been published to be in the order of $6.6 \mathrm{~mm}$ (7). Hence, shortening of the muscle fibres, due to knee abduction, was approximately $2.5 \%$. Hind limb displacement mainly occurred within the first $20 \mathrm{~ms}$ after the onset of stimulation, therefore, the fibre shortening velocity was at least $125 \% \cdot \mathrm{s}^{-1}$. 
Lengthening (2.5\%) occurred during $40 \mathrm{~ms}$ of the relaxation phase, corresponding with a $62.5 \% \cdot \mathrm{s}^{-1}$ lengthening velocity. These measured shortening of the muscletendon complex after onset of contraction and measured lengthening during relaxation had been prescribed to the mathematical model. A tetanic contraction simulated with the measured hind limb displacement showed a prolonged 10-50\% rise time compared to the simulation without this displacement ( $15.0 \mathrm{~ms}$ vs. $13.5 \mathrm{~ms}$, respectively). The calculated $1 / 2$-relaxation time with a hind-limb displacement amounted to $12.7 \mathrm{~ms}$ and was shorter than the $1 / 2$-relaxation time without the displacement $(14.3 \mathrm{~ms})$. Hence, the effect of displacements on temporal parameters due to non-absolute fixation is not negligible, but remains within acceptable limits. Maximal torque generation is not affected by this prolonged torque built up, because duration of the tetanus lasts sufficiently long to reach the maximal torque value.

As mentioned before, torque generated by a muscle complex is influenced by the shortening velocity as well as the initial length of the muscle complex $(9,11,12)$. A shortening of the muscle complex by $2.5 \%$ will only marginally affect the maximally generated muscle torque via the force-length relationship, because optimal ankle angle, i.e., ankle angle at maximal torque, was determined in the contracted condition.

In the validation procedure of the fixation system, plantar flexors were studied because they were expected to cause the largest displacements for two reasons. First, their torque output is higher compared to dorsal flexors $(11.8 \pm 1.6 \mathrm{mN} \cdot \mathrm{m}$ vs. $3.2 \pm 0.4$ $\mathrm{mN} \cdot \mathrm{m}$, see below). Second, the plantar flexor complex is bi-articular, i.e., its spans both the ankle and knee joints. For these reasons, contractions of the plantar muscle complex probably cause a larger knee and ankle displacement than the mono-articular dorsal flexors.

\subsubsection{Effect of eccentricity on measured torque}

The effect of eccentricity on muscle torque is shown in figure 3.4. These data show a small coefficient of variation in maximal torque of dorsal $(1.5 \%)$ and plantar muscles $(3.0 \%)$. Hence, the conclusion can be drawn that longitudinal eccentricity between ankle joint and apparatus axis during isometric contractions exerts only a minor effect on measured torque in the present experimental set-up. 
figure 3.4 Measured torque during stimulation of dorsal (closed circles) and plantar (open squares) muscle complexes as a function of eccentricity.

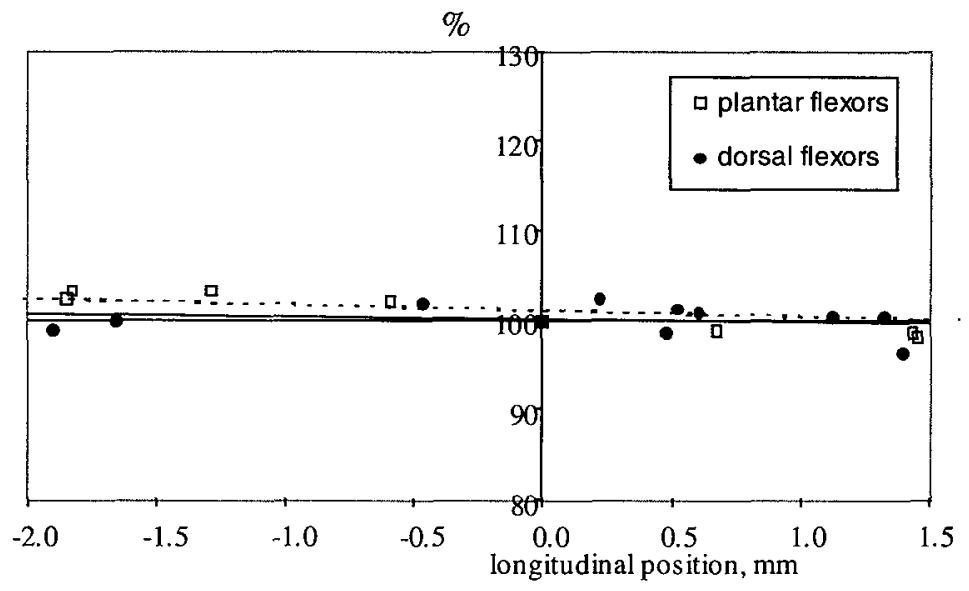

Solid and dashed lines represent the regression lines of dorsal and plantar flexors, respectively. Offset position $(0.0 \mathrm{~mm}$ at $\mathbf{x}$-axis) is estimated by visually aligning the ankle axis and the apparatus axis. Data are expressed as percentage of torque at offset position.

\subsubsection{Determination of contractile muscle parameters}

Tetanic muscle torque of plantar and dorsal flexors was $11.8 \pm 1.6 \mathrm{mN} \cdot \mathrm{m}$ and $3.2 \pm 0.4 \mathrm{mN} \cdot \mathrm{m}(\mathrm{n}=6)$, respectively (table 3.1$)$. Twitch torque of plantar and dorsal flexors amounted to $3.8 \pm 0.4 \mathrm{mN} \cdot \mathrm{m}$ and $1.0 \pm 0.2 \mathrm{mN} \cdot \mathrm{m}$, respectively. Representative patterns of tetanic and twitch contractions of the plantar and dorsal muscle complexes are presented in figures $3.5 \mathrm{a}$ and $3.5 \mathrm{~b}$, respectively.

Using histological sections, Ashton-Miller and colleagues (1) measured plantar and dorsal flexor lever arms of $2.1 \mathrm{~mm}$ and $1.0 \mathrm{~mm}$, respectively. Using these values and the cross-sectional areas (CSA) published by Burkholder et al. (7), the maximal tension of the plantar and dorsal flexors is estimated at $0.32 \pm 0.04 \mathrm{~N} \cdot \mathrm{mm}^{-2}$ and $0.41 \pm$ $0.06 \mathrm{~N} \cdot \mathrm{mm}^{-2}$, respectively. These tension values are in the same range as determined by Brooks and Faulkner $(5,6)$. 
figure 3.5 Representative tetanic and twitch isometric torque of mouse plantar flexors (a) and dorsal flexors (b).
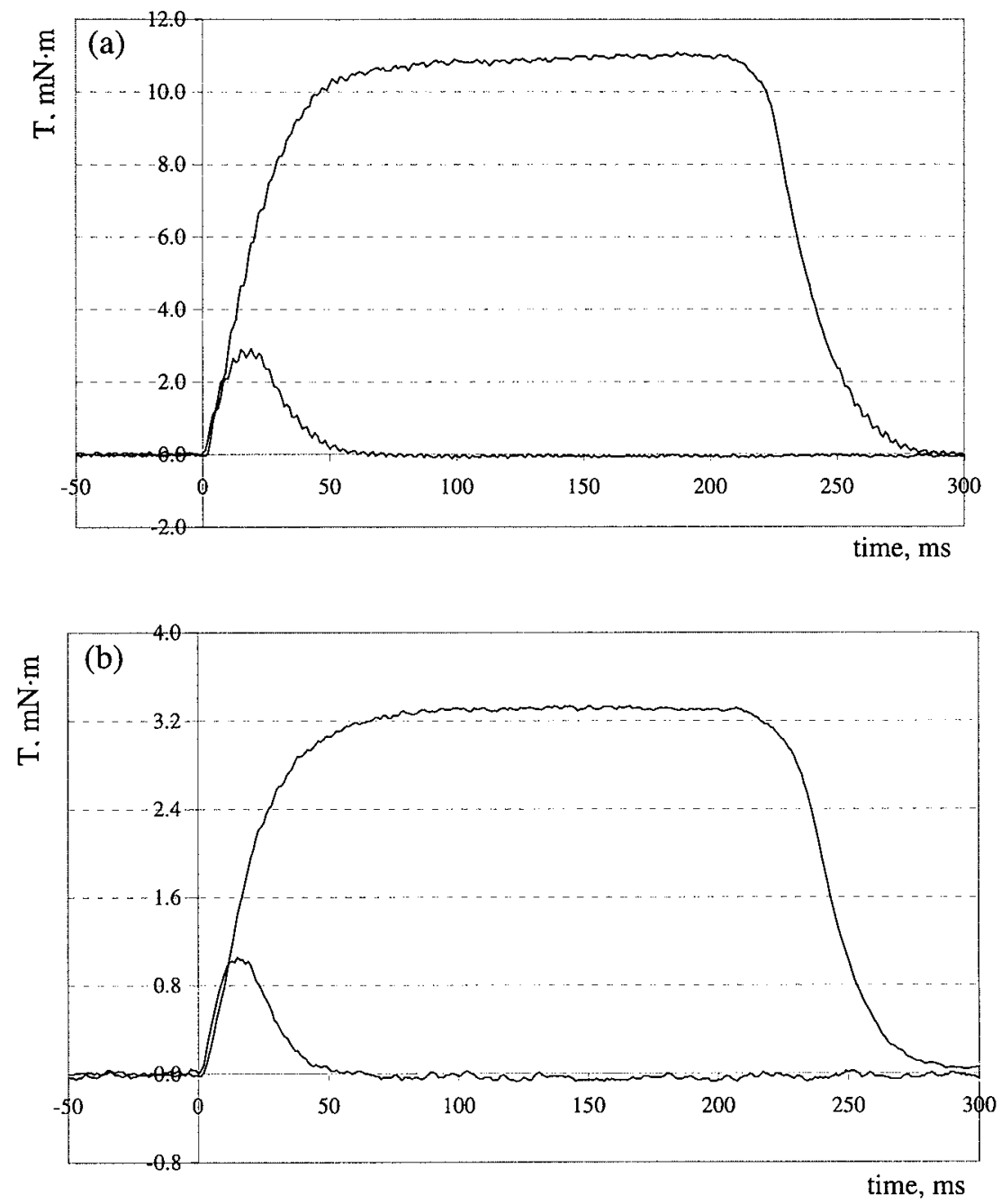

Ashton-Miller et al. (1) performed pioneering studies with respect to the analysis of muscular activity of intact mice. Their set-up comprises a pair of small strain gauges to determine muscle torque. Taken their approach as a starting point, we designed an experimental device, with which the muscle torque around the ankle joint is determined by monitoring minor deformation of torsion springs. On theoretical grounds, it was inferred that assessment of muscle torque with strain gauges or with a torque transducer as used in our set-up does not result in large differences in the signal-to-noise ratio. 
Muscle torque assessment with strain gauges, however, results in a more compliant measurement set-up. The lower compliance of our experimental model, in combination with an improved fixation system, most likely provides a more accurate determination of the temporal parameters of the tetanic contraction compared to the Ashton-Miller set-up. The advantage of the Ashton-Miller set-up is that concentric (muscle complex shortening) and eccentric (muscle complex lengthening) properties of the skeletal muscle can be assessed, whereas our set-up is not appropriate for measuring these parameters.

table 3.1 Tetanic torque characteristics during maximal isometric contractions of plantar and dorsal mouse hind limb flexors.

\begin{tabular}{lccc}
\hline & tetanic torque, $m N \cdot m$ & $10-50 \%$ rise time, $m s$ & $\begin{array}{c}1 / 2 \text {-relaxation time } \\
(50 \%-25 \%), m s\end{array}$ \\
\cline { 2 - 4 } & $11.8 \pm 1.6^{* *}$ & $14.9 \pm 0.6^{*}$ & $13.4 \pm 1.2^{*}$ \\
plantar flexors $(100 \mathrm{~Hz})$ & $3.2 \pm 0.4$ & $11.8 \pm 2.0$ & $10.4 \pm 1.1$ \\
\hline
\end{tabular}

Data are expressed as mean $\pm s d(n=6)$. Significant differences between dorsal and plantar flexors are indicated $(*: \mathrm{p}<0.05, * *: \mathrm{p}<0.01)$.

Tetanic $10-50 \%$ rise time and 1/2-relaxation time was significantly longer in the mouse plantar flexor muscles than in a dorsal flexor complex. The higher proportion of fast twitch muscle fibres (type IIa and IIb) most likely causes these differences between the dorsal and plantar flexor complexes (7).

The $1 / 2$-relaxation time of plantar flexors $(13.4 \pm 1.2 \mathrm{~ms})$ measured in the present experimental set-up was longer than measured in studies using dissected muscles. For instance, Steeghs and colleagues measured a 1/2-relaxation time of $5.0 \pm 1.9 \mathrm{~ms}$ in an isolated medialis gastrocnemius muscle preparation (15). Since the total plantar flexor complex has a higher proportion of slow twitch fibres than the dissected gastrocnemius medialis muscle, the contraction velocity of the plantar muscle complex is most likely slower than of a dissected muscle (7). Furthermore, dissected muscle studies are performed with completely or partly removed distal tendons, resulting in a less compliant muscle structure. This decrease in compliance might offer an additional explanation for the shortening of the 1/2-relaxation time of isolated muscles compared to the total plantar flexor complex.

In summary, the present experimental model is designed for accurate and sensitive in situ measurements of torque during isometric contractions of intact dorsal and plantar muscle complexes of mice. Furthermore, the novel fixation system resulted in reliable assessments of contraction parameters of mice ankle flexors. 


\section{5 - Acknowledgement}

We gratefully acknowledge the helpful advice of Dr. M.K.C. Hesselink, Department of Movement Sciences, Maastricht University, The Netherlands and the technical support of Ir. E. Dekkers of the Eindhoven University of Technology, The Netherlands. 


\section{6 - References}

1. Ashton-Miller J.A., He Y. Kadhiresan V.A., McCubbrey D.A., Faulkner J.A. (1992) An apparatus to measure in situ biomechanical behavior of dorsi- and plantar flexors of mouse ankle. J. Appl. Physiol. 72, 1205-1211

2. Barratta R.V., Solomonow M., Best R., Zembo M., Ambrosia R. (1995) Force-velocity relations of nine load-moving skeletal muscles. Med. Biol. Eng. Comput. 33, 537-544

3. Barclay C.J., Arnold P.D., Gibbs, C.L. (1995) Fatigue and heat production in repeated contractions of mouse skeletal muscle. J. Physiol. 488, 741-752

4. Barclay C.J., Constable J.K., Gibbs C.L. (1993) Energetics of fast- and slow-twitch muscles of the mouse. J. Physiol. 472, 61-80

5. Brooks S.V., Faulkner J.A., McCubbrey D.A. (1990) Power outputs of fast and slow skeletal muscles of mice. J. Appl. Physiol. 68, 1282-1285

6. Brooks S.V., Faulkner J.A. (1991) Forces and powers of slow and fast skeletal muscle in mice during repeated contractions. J. Physiol. 436, 701-710

7. Burkholder T.J., Fingado B., Baron S., Lieber R.L. (1994) Relationship between muscle fibre types and sizes and muscle architectural properties in the mouse hindlimb. $J$. Morphol. 221, 177-190

8. Edwards R.H.T., Hill D.K., Jones D.A. (1975) Metabolic changes associated with the slowing of relaxation in fatigued mouse muscle. J. Physiol. 251, 287-301

9. Gordon A.M., Huxley A.F., Julian F.J. (1966) The variation in isometric tension with sarcomere length in vertebrate muscles fibres. J. Physiol. 184, 170-192

10. Griffiths R.I. (1991) Shortening of muscle fibres during stretch of the active cat medial gastrocnemius muscle: the role of tendon compliance. J. Physiol. 436, 219-236

11. James R.S., Altringham J.D., Goldspink D.F. (1995) The mechanical properties of fast and slow twitch muscles of the mouse in relation to their locomotory function. $J$. Exp. Biol. 198, 491-502

12. Lieber R.L. (1997) Muscle fiber length and moments arm coordination during dorsi- and plantar flexion in the mouse hind limb. Acta Anat. 159, 84-89

13. Maenhout M., Drost M.R., Hesselink, M.K.C., Oomens, C.W.J. (1999) Parameter identification of a distribution-moment model describing the active behavior of the tibialis anterior muscle of the rat. ASME BED, 42, 387-388

14. Meanhout M., Hesselink M.K.C., Oomens C.W.J., Drost M.R. Parameter identification of a distribution-moment aproximated two-state Huxley model of the rat tibialis anterior muscle. In: Muscle mechanics: from molecules to function, Herzog W (ed), in press

15. Steeghs K., Benders A., Oerlemans F., De Haan A., Heerschap A., Ruitenbeek W., Jost C., Van Deursen J., Perryman B., Pette D., Bruckwilder M., Koudijs J., Jap P., Veerkamp J., Wieringa B. (1997) Altered $\mathrm{Ca}^{2+}$ responses in muscles with combined mitochondrial and cytosolic creatine kinase deficiencies. Cell 89, 93-103

16. Van Donkelaar C.C., Willems P.J.B., Muijtjens A.M.M., Drost M.R. (1999) Skeletal muscle transverse strain during isometric contraction at different lengths. J. Biomech., 32, 755-762

17. Wilkie D.R. (1950) The relation between force and velocity in human muscle. J. Physiol. $110,249-280$

18. Zahalak G.I. (1981) A distribution-moment approximation for kinetic theories of muscular contraction. Math. Biosc. 55, 89-114

19. Zahalak G.I., Ma S. (1990) Muscle activation and contraction: constitutive relations based directly on cross-bridge kinetics. J. Biomech. Eng. 112, 52-62 


\section{Chapter 4}

\section{N SITU ASSESSMENT OF SHORTENING AND LENGTHENING CONTRACTION PROPERTIES OF HINDLIMB ANKLE FLEXORS OF INTACT MOUSE.}

M. Gorselink, M.R. Drost, J., de Louw, P.J.B., Willems, M.K.C. Hesselink, E.C.A. Dekkers, G.J. van der Vusse, (2000) Eur. J. Physiol., Instruments and Techniques, in press

Description of the mouse ergometer for assessment of shortening and lengthening properties of intact dorsal and plantar flexors of the mouse. 


\section{1 - Summary}

The availability of animal models with disrupted genes resulted in an increased necessity of small-scale measurement devices. Recently, we have developed an experimental device to assess in situ mechanical properties of isometric contractions of intact muscle complexes of the mouse (1). Although this set-up provides valuable information on muscle mechanical performance, it is not appropriate for determining contractile properties during shortening and lengthening contractions. Therefore, an experimental set-up for assessment of shortening and lengthening contractile properties of intact plantar and dorsal flexors of the mouse has been described and evaluated. The current through a custom-built low inertia servomotor was measured to assess contractile muscular torque ranging from -50 to $50 \mathrm{mN} \cdot \mathrm{m}$.

Evaluation of the fixation procedure of the animal to the set-up via 3-D monitoring of the muscle-tendon complex length showed that the additional shortening in length due to a contraction with maximal torque output exerts only minor effects on assessed torque. Furthermore, it was found that the misalignment of the axis of rotation of the set-up relative to the axis of rotation in the ankle joint, i.e., eccentricity, during a routine experiment was estimated to be less than $1.0 \mathrm{~mm}$, and hence, does not influence the measured torque output in our experimental set-up.

Peak power per unit muscle mass (mean $\pm \mathrm{sd}$ ) of intact dorsal and plantar flexors amounted to $0.27 \pm 0.02 \mathrm{~W} \cdot \mathrm{g}^{-1}$ and $0.19 \pm 0.03 \mathrm{~W} \cdot \mathrm{g}^{-1}$, respectively. The angular velocity at maximal peak power generated by the dorsal flexor complex and the plantar flexor complex amounted to $1100 \pm 190^{\circ} \cdot \mathrm{s}^{-1}$ and $700 \pm 90^{\circ} \cdot \mathrm{s}^{-1}$, respectively.

Keywords: mice, in situ, skeletal muscle, shortening contraction, contractile work 


\section{2 - Introduction}

Advances in the field of molecular genetics have provided powerful tools like transgenic and knockout mice, for a direct approach to study the interaction of mechanical functioning and skeletal muscle energy metabolism. The availability of animal models with disrupted genes, encoding for proteins making part of the contractile machinery or being involved in energy conversion, resulted in an increased necessity of small-scale measurement devices. Recently, we have developed an experimental device to assess in situ mechanical properties of isometric contractions of intact dorsal and plantar muscle complexes of the mouse (1). Although this set-up provides valuable information on muscle mechanical performance, it is not appropriate for determining contractile properties during shortening and lengthening contractions of the murine hindlimb ankle flexors. Pioneering studies in assessment of dynamic properties of intact murine hind limb flexors were performed by Ashton-Miller and colleagues (2). With their experimental set-up and our isometric measurement device as starting points, we have designed an experimental device for the assessment of shortening and lengthening contraction properties of intact mouse ankle flexors.

For meaningful measurement of dynamic contractile properties, the experimental device must be appropriate to assess muscular performance within the physiological range of locomotion of the mouse, e.g. stride frequency ranging from 1 to $10 \mathrm{~Hz}$, and maximal ankle flexion and extension amplitude of $40^{\circ}(2-5)$. Potential complications of measuring contractile properties during shortening and lengthening contractions include the fixation of the mouse to the experimental set-up and misalignment of the axis of rotation of the set-up relative to the axis of rotation in the ankle joint, i.e., eccentricity. Due to non-rigid fixation, displacement of the hind limb during the contraction will shorten the muscle-tendon complex, and, hence, affect muscular performance through the force-length and force-velocity relationships (6-8). Furthermore, we have previously shown that eccentricity between the ankle axis and the measurement shaft has only minor effects on contractile characteristics during isometric contraction (1). The effects of eccentricity during shortening contractions have not been published, but on theoretical basis, one may assume that eccentricity particularly during shortening and lengthening contractions will introduce an additional torque, due to inertial forces on the (lower) limbs or to forces on the knee fixation if a rigid knee fixation is used.

The main aim of the present study was to describe and evaluate a measurement device for assessment of in situ shortening and lengthening properties of the ankle flexors in an intact mouse. The second aim was to quantify the effects of non-rigid fixation of the hindlimb of the mouse on assessed torque during shortening contractions. 
The third aim of this paper was to quantify the effects of eccentricity between the rotation axis of the ankle joint and the axis of the measurement device on torque measured during shortening contractions. Finally, this study aims to determine a set of mechanical parameters for description of lengthening and shortening contractions of the intact dorsal and plantar flexors of the mouse, and compare them to data available in literature. Mechanical performance was characterised in terms of the generated muscle torque, torque-angular velocity relationship, Hill parameters, peak power, and maximal and optimal angular velocity of both the dorsal and plantar flexor muscles.

\section{3 - Materials and Methods}

\subsubsection{Apparatus description, torque measurement principles and definitions}

The entire experimental set-up consists of a thermostatic platform, hip fixation unit, the novel ergometer for assessment of the muscle-tendon complex properties during shortening and lengthening contractions and the isometric dynamometer for assessment of the contractile properties during isometric contractions (1). The muscletendon complex will be further denoted as 'muscle complex' in this paper.

The shaft $(\varnothing=4.0 \mathrm{~mm})$ of either the ergometer or the isometric dynamometer passes through a cavity $(\varnothing=4.5 \mathrm{~mm})$ in the platform, and is rigidly connected to the mouse foot. The ergometer (fig. 4.1) consists of a custom-built low inertia servomotor, with a built-in encoder (HP Encoder disc, HED 6120 and HP Encoder module HEDS 9000, Hewlett Packard, Amstelveen, The Netherlands) to monitor angular position, and an angular position control unit (PMAC processor, Delta-Tau-Data Systems, Northridge, CA, USA). Data collection was performed using an Apple Macintosh7100 PowerPC ${ }^{\oplus}$ with an 8 channel, 12 bits Lab-NB analogue-to-digital conversion board (National Instruments, Mopac Expwy, Austin, USA) and an electrical pulse generator (HSE 215/IZ, Freiburg, Germany).

The optical encoder monitors the shaft position with an accuracy of approximately $0.04^{\circ}$, i.e., 8192 -edge detection pulses. During an experiment, the shaft of the servomotor can be sinusoidally oscillated with a prescribed frequency $(0.01-25$ $\mathrm{Hz}$ ) and amplitude up to $45^{\circ}$. A PMAC board regulates the angular position of the servomotor shaft, and therefore the ankle angle, via a closed loop feed back system. The current through the servomotor is used to assess the torque output. Torques ranging from $-50 \mathrm{mN} \cdot \mathrm{m}$ and $+50 \mathrm{mN} \cdot \mathrm{m}$ can be measured. The precision at physiological oscillation frequencies was estimated at approximately $0.1 \mathrm{mN} \cdot \mathrm{m}$ and at $0.2 \mathrm{mN} \cdot \mathrm{m}$ at higher oscillation frequencies. 
figure 4.1 A schematic overview of the mouse ergometer for muscle torque and power assessment of the murine ankle flexors.

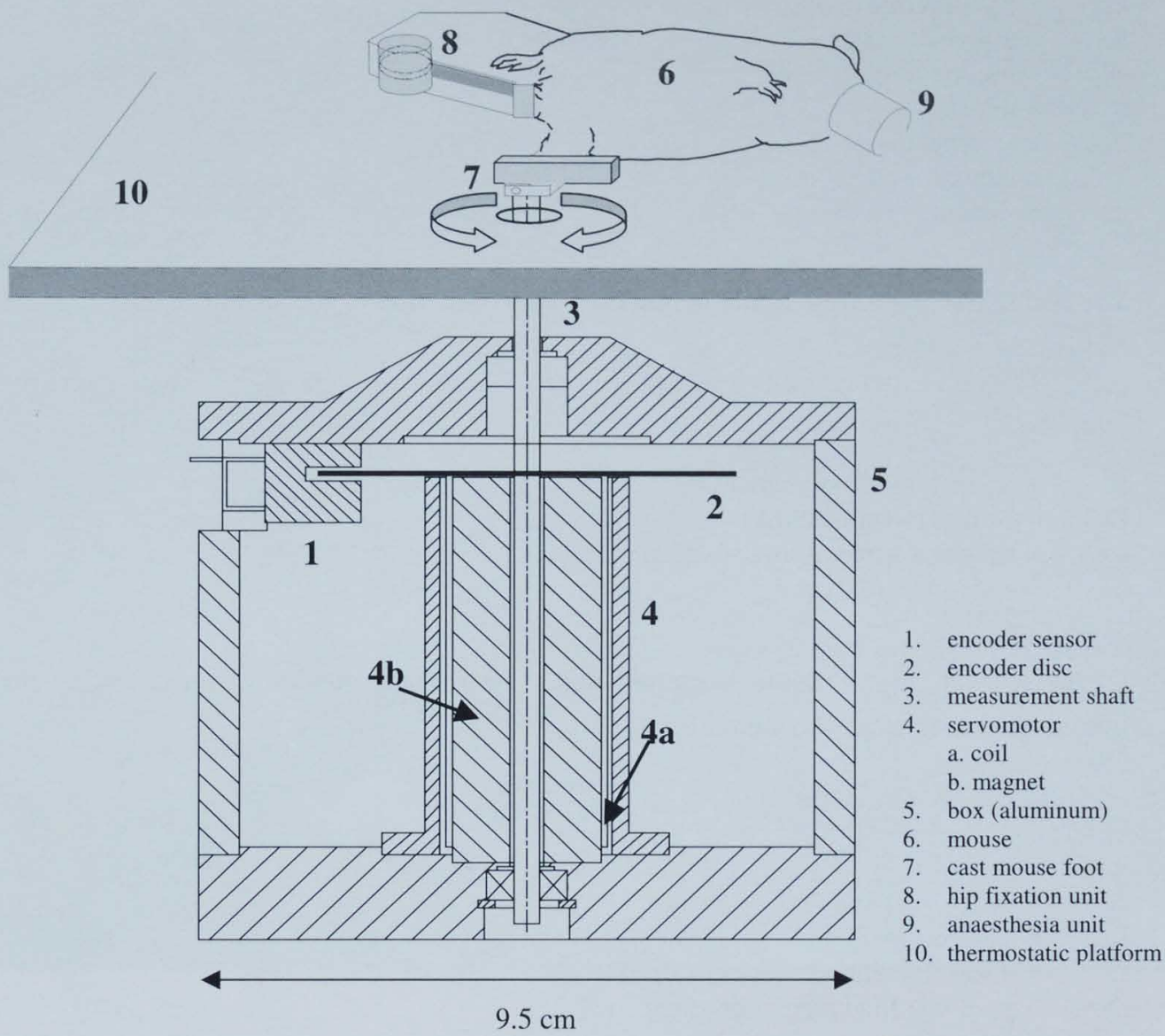

The figure presents a 3-D view of the mouse and the thermostatic platform, whereas a cross-section of the ergometer is given. The wires are not shown in this cross-section.

The motor consists of a shaft connected to a low inertia coil, which can rotate in a gap $(1.2 \mathrm{~mm})$ in a radially oriented magnetic field. The coil is connected with a pair of electric wires. The second law of motion (eqn. 4.1) on the coil plus shaft gives:

$$
J \cdot \frac{d^{2} \varphi}{d t^{2}}=-k \cdot \varphi-K_{0}+T_{\text {motor }}+T_{\text {ext }}-T_{f} \cdot \operatorname{sign}\left(\frac{d \varphi}{d t}\right)-d \cdot\left(\frac{d \varphi}{d t}\right)
$$


with

$\mathrm{d} \quad=$ damping coefficient $\left(\mathrm{N} \cdot \mathrm{m} \cdot \mathrm{s} \cdot \mathrm{rad}^{-1}\right)$

$\mathrm{J} \quad=$ moment of inertia of coil and shaft $\left(\mathrm{kg} \cdot \mathrm{m} \cdot \mathrm{s}^{-2}\right)$

$\mathrm{k} \quad=$ stiffness due to wire $\left(\mathrm{N} \cdot \mathrm{m} \cdot \mathrm{rad}^{-1}\right)$

$\mathrm{K}_{0} \quad=$ torque offset $(\mathrm{N} \cdot \mathrm{m})$

$\mathrm{T}_{\mathrm{f}} \quad=$ friction torque $(\mathrm{N} \cdot \mathrm{m})$

$\mathrm{t} \quad=$ time $(\mathrm{s})$

$\mathrm{T}_{\mathrm{ext}}=$ external torque $(\mathrm{N} \cdot \mathrm{m})$

$\mathrm{T}_{\text {motor }}=$ motor torque $(\mathrm{N} \cdot \mathrm{m})$

$\varphi \quad=$ shaft angle $(\mathrm{rad})$

The coil delivers a torque in the magnetic field as function of its current:

$$
T_{\text {motor }}=B \cdot I \cdot l \cdot r_{\mathrm{s}}
$$

with

$\mathrm{B} \quad=$ magnetic field strength $(\mathrm{T})$

I $=$ current though $\operatorname{coil}(\mathrm{A})$

$1 \quad=$ length of wire within coil $(5 \mathrm{~m})$

$\mathrm{r}_{\mathrm{s}} \quad=$ coil $\operatorname{radius}(0.01 \mathrm{~m})$

Dispersion at high motor currents and linear and quadratic terms of field inhomogeneities were accounted for:

$$
B=B_{0}+A \cdot I+C \cdot \varphi+D \cdot \varphi^{2}
$$

with
$\mathrm{A} \quad=$ field dispersion $\left(\mathrm{T} \cdot \mathrm{A}^{-1}\right)$
$\mathrm{B}_{\mathrm{o}} \quad=$ basal magnetic field strength $(\mathrm{T})$
$\mathrm{C}=$ linear field inhomogeneity $\left(\mathrm{T} \cdot \mathrm{rad}^{-1}\right)$
$\mathrm{D}=$ quadratic field inhomogeneity $\left(\mathrm{T} \cdot \mathrm{rad}^{-2}\right)$

It follows from equation 4.2 and 4.3 that,

$$
T_{\text {motor }}=\left(B_{0}+A \cdot I+C \cdot \varphi+D \cdot \varphi^{2}\right) \cdot I \cdot l \cdot r_{s}
$$

The constants were estimated in a series of experiments:

1) at low oscillation frequency $(0.02 \mathrm{~Hz})$ with four external loads $(0.3,4.2,8.1$, $16.2 \mathrm{mN} \cdot \mathrm{m}$ ) values taken at $\varphi=0: \mathrm{K}_{0}, \mathrm{~B}_{\mathrm{o}}, \mathrm{A}, \mathrm{T}_{\mathrm{f}}$;

2) at low oscillation frequency $(0.02 \mathrm{~Hz})$ without external load at non-zero angle: $\mathrm{k}, \mathrm{C}, \mathrm{D}$;

3) at high oscillation frequency $(22 \mathrm{~Hz}): \mathrm{g}, \mathrm{J}$. 
Five constants $\left(\mathrm{K}_{\mathrm{o}}, \mathrm{g}, \mathrm{C}, \mathrm{D}, \mathrm{T}_{\mathrm{f}}\right)$ could be neglected because their contribution to the torque was always less than $0.01 \mathrm{mN} \cdot \mathrm{m}$. Rewriting the formula with remaining nonzero coefficients, using voltage instead of current as output parameter (conversion constant $0.91 \mathrm{~V} \mathrm{~A}^{-1}$ ) and bringing $l$ and $r_{s}$ in the field strength coefficients gives:

$$
T_{e x t}=\left(B_{o}^{*}+A^{*} \cdot V\right) \cdot V+J \cdot\left(\frac{d^{2} \varphi}{d^{2}}\right)+k \cdot \varphi
$$

with

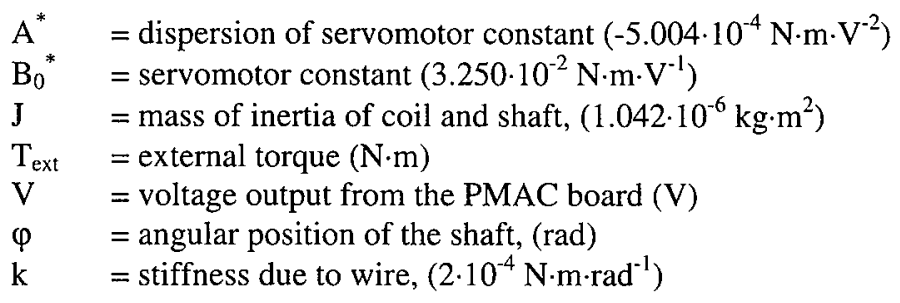

The angle $\varphi$ was filtered (5 point moving average) before numerical differentiation, as was the angular velocity to determine angular acceleration. To differentiate between inertial, frictional and passive torque of the muscle on the one hand and contractile torque on the other hand, passive (without electrical stimulation of the muscles) and active (with electrical stimulation of the muscles) oscillations were alternated. Contractile torque was determined by subtracting torque during passive oscillations from torque generated during active oscillations.

\subsubsection{Animal handling and muscle complex stimulation procedure}

All experiments were performed on 12 week old male mice (Swiss, $25.6 \pm 0.8$ $\mathrm{g}$ ), positioned on an aluminum platform maintained at $38.5 \pm 0.1{ }^{\circ} \mathrm{C}$. Mice were anaesthetised with Halothane (1.5-2.0\%) (Fluothane ${ }^{\circledR}$, Zeneca, Ridderkerk, The Netherlands) mixed with $\mathrm{O}_{2}$ and $\mathrm{N}_{2} \mathrm{O}\left(3: 1,4.0 \mathrm{l} \cdot \mathrm{min}^{-1}\right)$. All experimental procedures were approved by the Animal Care and Use Committee of the Maastricht University and complied with the principles of proper laboratory animal care.

Experimental procedures, such as fixation of the mouse and attachment of the electrode, were described recently (1). Briefly, the anaesthetised mouse was positioned on the thermostatic measurement platform and the foot was cast in a shoe made of two-component cement (Paladur, Heraus Kulzer GmbH, Wehrheim, Germany). The shoe was glued with cyano-acrylate (Bison, Goes, The Netherlands) to a shoe-fixation plate, which was rigidly connected to the apparatus shaft. The proximal part of the hindlimb was fixated to the external world through the hip fixation unit. 
In order to prevent knee abduction, the lateral condyle of the femur was supported by a small Teflon cube.

Plantar or dorsal muscle complexes were electrically stimulated via the tibial or peroneal nerve, respectively. After depilating the skin, a small incision in either the hollow or in the lateral part of the knee was made to obtain access to specific nerves. A bipolar platinum hook electrode, interpole distance $0.5 \mathrm{~mm}$, was carefully attached to the nerve and the muscle was stimulated using a pulse generator. Supra-maximal current and optimal ankle angle were determined with the isometric dynamometer (1). Supra-maximal stimulation current, necessary to obtain recruitment of all muscle fibres, was determined using 3 to 5 twitch contractions with increasing current. This supra-maximal current was used throughout the entire experiment. The electrode was removed and reattached in a more distal position, if the current exceeded $1.0 \mathrm{~mA}$. Ankle angle at which the contraction exerts maximal torque output was determined using 7 to 10 twitch contractions around the $90^{\circ}$ ankle angle in steps of $5^{\circ}$, and will be further denoted as the optimal ankle angle. Recovery period between twitches was 60 s. Upon these procedures, the electrode was fixated to the skin with cyano-acrylate glue to prevent electrode displacement during muscle contraction. Isometric contractions for both muscle complexes were obtained with a $150 \mathrm{~ms}$ pulse train, a 125 $\mathrm{Hz}$ stimulation frequency, and pulse duration of $0.5 \mathrm{~ms}$. Upon these measurements, the isometric dynamometer was replaced by the ergometer. In this procedure, the ankle angle was maintained at its optimal value.

\subsubsection{Experimental procedure to validate the fixation system}

Measurements of alterations in plantar flexor muscle length due to the non-rigid fixation as applied in the present set-up were restricted to plantar flexors since they were expected to cause the largest displacements of the knee joint for two reasons. First, their maximal force is higher than that generated by the dorsal flexors (1). Second, most of the plantar flexor complex is bi-articular, i.e., it spans both the ankle and knee joints.

Changes in length of the muscle complex during shortening contractions were monitored with a 3-D marker tracking system $(n=1)$. Briefly, two synchronised $25 \mathrm{~Hz}$ CCD (400 x 512 pixels) cameras (MX5, Adimec, Amsterdam, The Netherlands) were positioned above the contracting muscle. Odd and even video images $(50 \mathrm{~Hz}, 200 \mathrm{x}$ 512 pixels) were separated and real-time digitised (9). The medial condyle of the femur was surgically exposed, subsequently a shallow hole was drilled near the insertion of the medial gastrocnemius muscle. A small fluorescent polystyrene sphere $(\varnothing=0.4 \mathrm{~mm}$ ) (Bang Laboratories, Inc. Fisshers, Carmel, Indiana, USA) was attached in this hollow with cyano-acrylate glue. 
A second sphere was attached to the cast mouse foot at the sagital plane projection of the insertion of the Achilles tendon. Geometrical marker centroids of the spheres were determined from digitised image frames using TIMwin 1.3 (TEA, Dordrecht, The Netherlands). Post-processing of the marker tracks was performed with Matlab 5.3 (The Math Works, Inc., Natick, Massachusetts, USA). The distance between the spheres on the cast foot and the femur was defined as muscle length. The alterations in muscle length due to non-rigid fixation do affect the actual shortening velocity. Therefore, the differences in muscle length and the differences in velocity between a passive and active oscillation were computed at $1,2,4$ and $8 \mathrm{~Hz}$ oscillation frequencies at an amplitude of $20^{\circ}$.

\subsubsection{Experimental procedure to assess the effect of eccentricity on torque}

Eccentricity is defined as the misalignment between the apparatus shaft and the rotation axis of the ankle joint. The eccentricity in the length direction of the mouse foot (longitudinal axis) was varied with a specially constructed shoe plate, allowing a manual longitudinal displacement of the mouse foot $(n=1)$ (1). Two fluorescent polystyrene spheres were attached to the cast mouse foot and exact longitudinal displacements between successive measurements were monitored with a 2-D marker tracking system (9). Visual alignment between ankle and apparatus shaft was used as offset estimation. The eccentricity was randomly varied between approximately 2.5 $\mathrm{mm}$ in the toe direction and approximately $6.0 \mathrm{~mm}$ in the heel direction of the mouse foot. The effect of eccentricity on generated torque during active and passive strokes was measured at 1 and $8 \mathrm{~Hz}$ oscillation frequencies with an amplitude of $20^{\circ}$.

\subsubsection{Assessment of contractile properties}

Shortening and lengthening contractile properties were assessed at supramaximal stimulation current $(n=4)$. Ankle flexion and extension was performed around the optimal ankle angle. Mean angular velocities were varied between $20.0^{\circ} \cdot \mathrm{s}^{-1}$ and $3000^{\circ} \cdot \mathrm{s}^{-1}$ by ranging the oscillation frequencies and amplitude. Stimulation frequency was increased from $125 \mathrm{~Hz}$ at $12.5^{0} \cdot \mathrm{s}^{-1}$ towards $300 \mathrm{~Hz}$ at $650^{0} \cdot \mathrm{s}^{-1}$ and higher oscillation velocities. The torque-angular velocity relationship of the plantar and dorsal flexors was described as the contractile torque as a function of its angular velocity both determined at optimal ankle angle. The shortening part of the torque-angular velocity relation was fitted via a least-squares method to a hyperbola described by the Hill equation $\left(T_{0}, a, b\right)(10)$. For each muscle complex, power-angular velocity relationship was assessed as the product of the calculated contractile torque and the angular velocity. 
Optimal angular velocity $\left(\mathrm{V}_{\mathrm{opt}}\right)$ was defined as the angular velocity at which the power reached its maximum. Maximal angular velocity $\left(\mathrm{V}_{\max }\right)$ was estimated as the intercept of the Hill curve with the angular velocity axis.

\subsubsection{Statistics}

Values are presented as mean \pm sd. Differences between plantar and dorsal flexors in mechanical parameters were analysed using non-parametric Mann-Whitney U test. Differences were considered significant if $\mathrm{p}<0.05$. SPSS 9.0 (SPSS Benelux, Gorinchem, The Netherlands) was used for statistical analyses.

\section{4 - Results}

\subsubsection{Description and evaluation of the measurement device}

Figure 4.2 shows a representative example of a shortening contraction of the plantar flexors. Panel A describes the pattern of the sinusoidal angular course $(1 \mathrm{~Hz}$, $20^{\circ}$ ). Panel $\mathrm{B}$ shows the passive torque, whereas panel $\mathrm{C}$ depicts the active torque. Panel $\mathrm{D}$ shows the contractile torque, computed by subtraction of the passive torque from the active torque.

\subsubsection{Validation of fixation system}

The plantar flexor complex length, at optimal angle of approximately $15^{\circ}$ dorsal flexion, was estimated at $19.69 \mathrm{~mm}$. Alterations in plantar flexor length, due to nonrigid fixation were maximal at $1 \mathrm{~Hz}$ oscillation frequency. A passive oscillation revealed that muscle length at $35^{\circ}$ dorsal flexion was increased by $2.8 \%$, and at $5^{\circ}$ plantar flexion decreased by $3.8 \%$. Differences in muscle length between a passive and active stroke were monitored. The maximal difference amounted to $0.21 \mathrm{~mm}$ at 1 $\mathrm{Hz}$ oscillation frequency. With increasing velocity, differences in muscle length between the passive and active strokes decreased, e.g. $0.08 \mathrm{~mm}$ at $8 \mathrm{~Hz}$ oscillation frequency. The difference in muscle shortening velocity between a passive stroke and an active stroke at $1 \mathrm{~Hz}$ oscillation frequency is depicted in figure 4.3. This difference was less than $0.3 \mathrm{~mm} \cdot \mathrm{s}^{-1}$ at optimal angle and amounted up to maximally $-7.6 \mathrm{~mm} \cdot \mathrm{s}^{-1}$ and $3.0 \mathrm{~mm} \cdot \mathrm{s}^{-1}$ during torque build up and relaxation, respectively. 
figure 4.2 Representative example of angular course (A), passive torque (B), active torque (C), and contractile torque (D) during $1.0 \mathrm{~Hz}$ oscillation frequency and $20^{\circ}$ amplitude of the murine plantar flexors relative to optimal angle ( $15^{\circ}$ dorsal flexion).

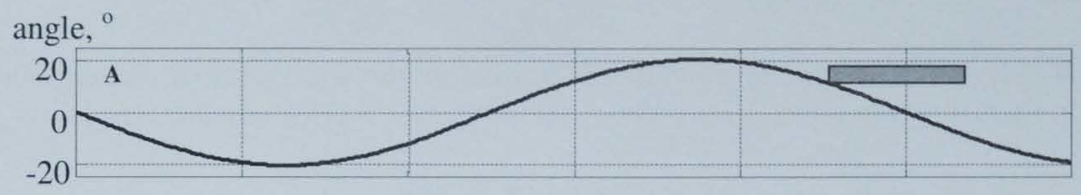

$\mathrm{T}, \mathrm{mN} \cdot \mathrm{m}$

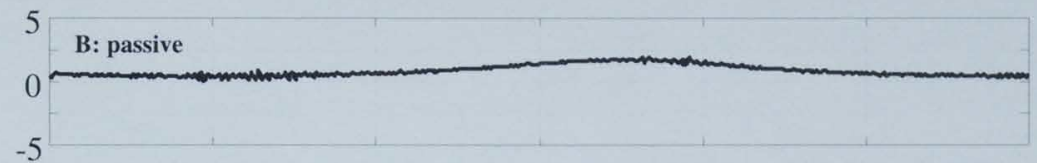

$\mathrm{T}, \mathrm{mN} \cdot \mathrm{m}$

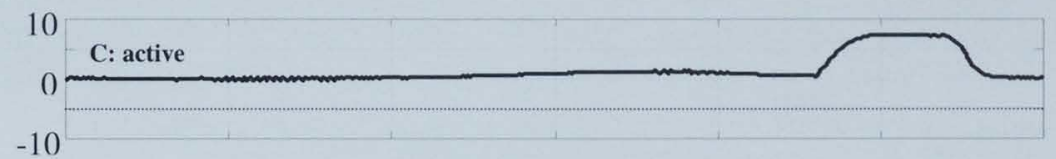

$\mathrm{T}, \mathrm{mN} \cdot \mathrm{m}$

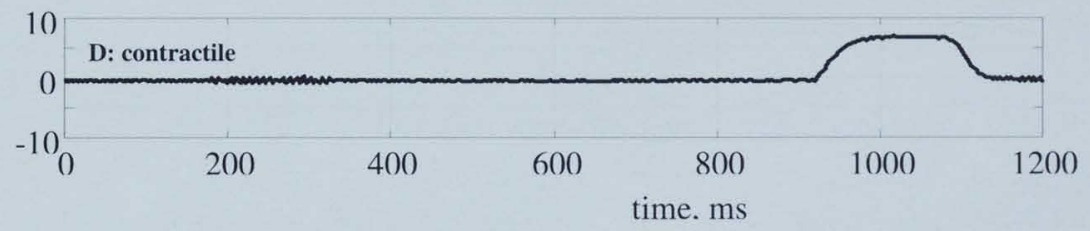

Negative and positive direction of the angular course corresponds with plantar and dorsal flexion, respectively. The phase of electrical stimulation is indicated in panel A by a shaded, horizontal bar.

figure 4.3 Differences in velocity between a passive and an active stroke of the plantar flexor of the mouse.

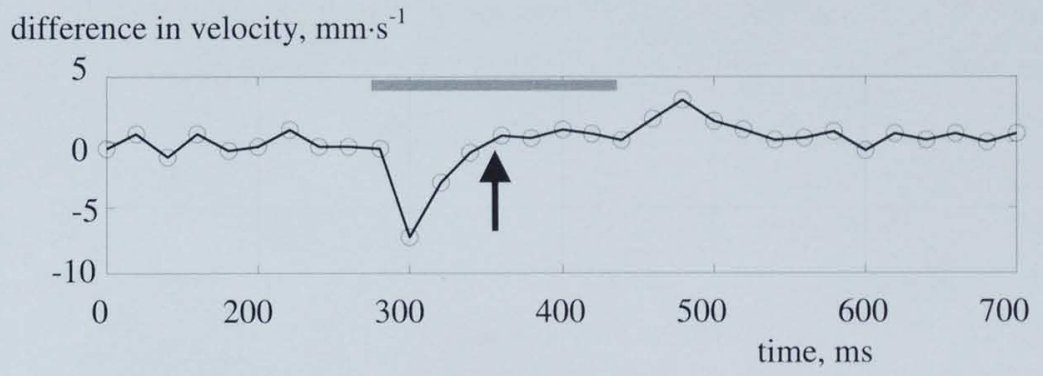

The shaded horizontal bar indicates the time interval of electrical stimulation of the muscle complex, whereas the arrow indicates the time point at which the ankle angle exerts its optimal value $(n=1)$. 


\subsubsection{Effect of eccentricity on assessed torque}

Effects of eccentricity at 1 and $8 \mathrm{~Hz}$ oscillation frequencies on passive and contractile torque are depicted in figure 4.4 and 4.5 , respectively.

figure 4.4 Effects of eccentricity on maximal passive torque at $20^{\circ}$ oscillation amplitude and oscillation frequencies of either $1 \mathrm{~Hz}$ (upper panel) and or $8 \mathrm{~Hz}$ (lower panel) $(\mathrm{n}=1)$.

\section{$1 \mathrm{~Hz}$ : passive torque}

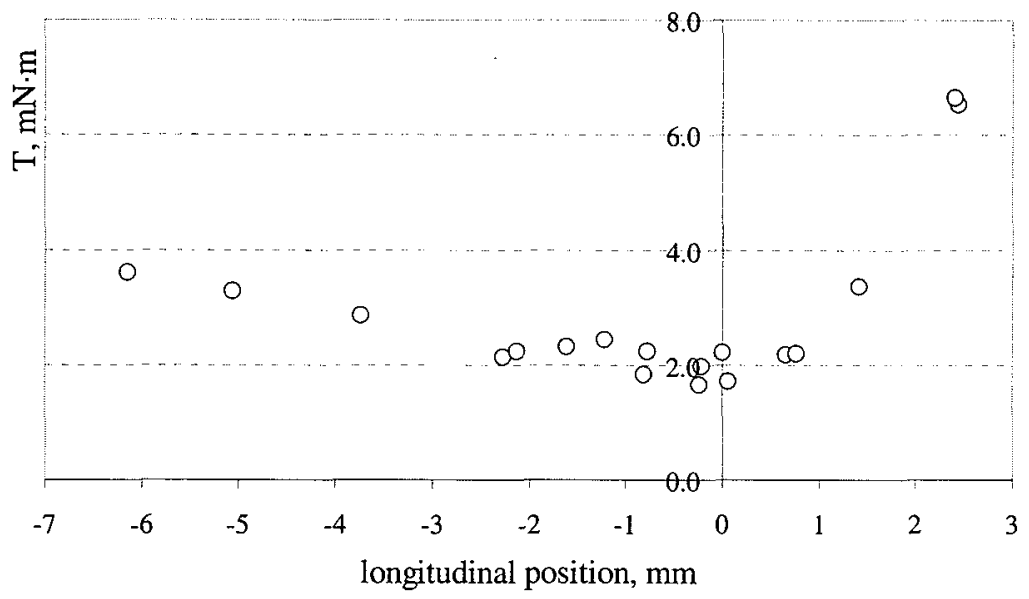

8 Hz: passive torque

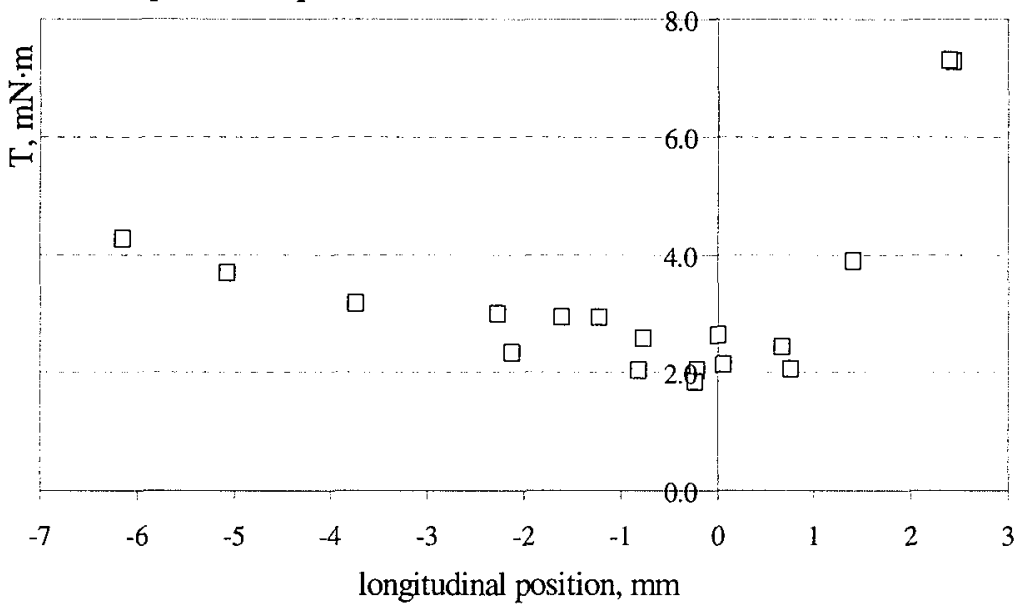

Passive torque at 1 and $8 \mathrm{~Hz}$ oscillation frequencies was affected when eccentricity in the heel direction exceeds $4.0 \mathrm{~mm}$ from visually adjusted optimal alignment and 1.5 $\mathrm{mm}$ in the toe direction. 
The effect of eccentricity on the contractile torque during an active stroke at $1 \mathrm{~Hz}$ oscillation frequency is of negligible magnitude. Contractile torque at $8 \mathrm{~Hz}$ oscillation frequency, however, increased at eccentricity distances exceeding $4.0 \mathrm{~mm}$ in the heel direction, and decreased at an eccentricity larger than about $2.0 \mathrm{~mm}$ in the toe direction.

figure 4.5 Effects of eccentricity on maximal contractile muscle torque assessed at optimal ankle angle during $20^{\circ}$ oscillation amplitude with a oscillation frequencies of either $1 \mathrm{~Hz}$ (upper panel) or $8 \mathrm{~Hz}$ (lower panel) $(\mathrm{n}=1)$.

$1 \mathrm{~Hz}$ : contractile muscle torque

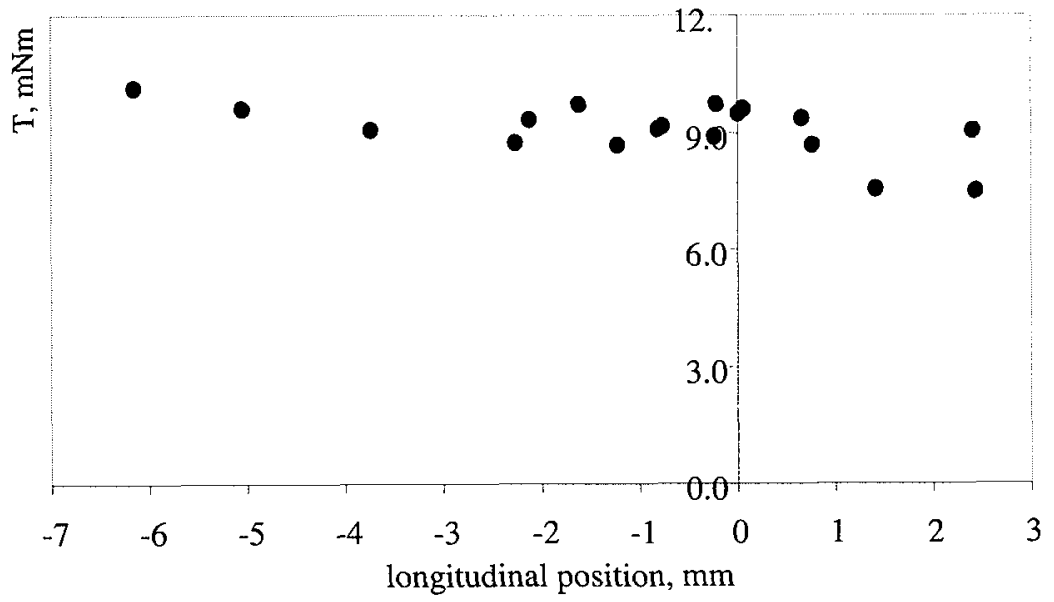

$8 \mathrm{~Hz}$ : contractile muscle torque

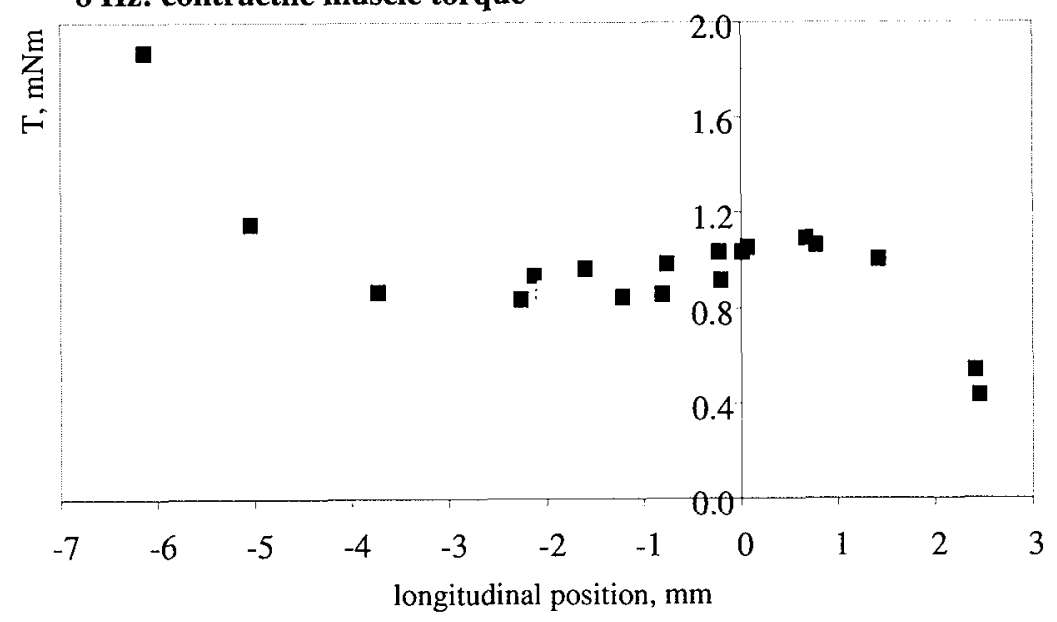


figure 4.6 Representative shortening (top panel) and lengthening (middle panel) contractile torque during $1.0 \mathrm{~Hz}$ oscillation frequency generated by the murine plantar flexors.

\section{$\mathrm{T}, \mathrm{mN} \cdot \mathrm{m}$ [ shortening]}

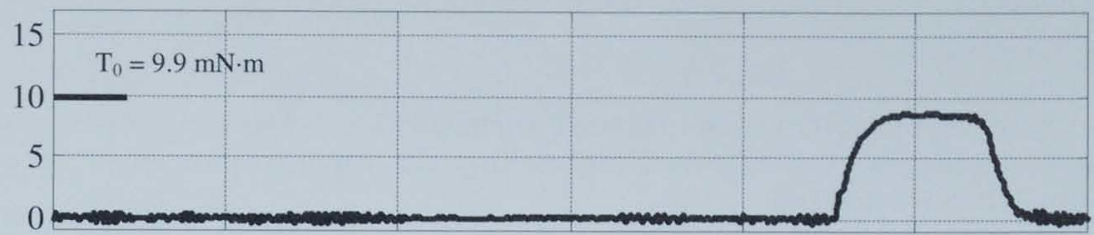

$\mathrm{T}, \mathrm{mN} \cdot \mathrm{m}$ [lengthening]
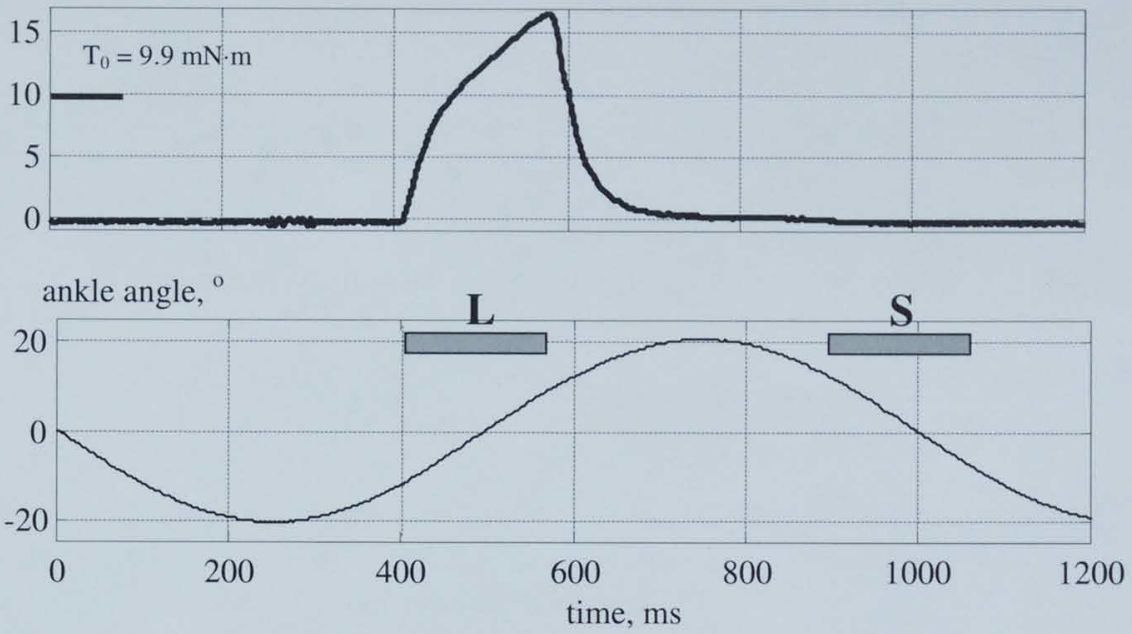

Lower panel shows the ankle angle $\left({ }^{\circ}\right)$ relative to optimal angle $\left(15^{\circ}\right.$ dorsal flexion). Negative and positive direction corresponds with plantar and dorsal flexion, respectively. S (shortening) or L (lengthening) indicates the phase of electrical stimulation of the plantar flexors. Maximal isometric torque is indicated by $\mathrm{T}_{0}$ (horizontal bar).

\subsubsection{Assessment of contractile properties}

Representative shortening and lengthening contractions at $1 \mathrm{~Hz}$ and $20^{\circ}$ amplitude during the angular oscillation of the plantar and dorsal flexor complex are presented in figure 4.6 and 4.7 , respectively. The upper panel of both figures shows the contractile torque of representative shortening (S) contractions of the plantar and dorsal flexors, whereas the middle panel shows contractile torque from the lengthening (L) contractions. The lower panel shows the phase of stimulation for either shortening or a lengthening contraction. 
figure 4.7 Representative shortening (top panel) and lengthening (middle panel) contractile torque during $1.0 \mathrm{~Hz}$ oscillation frequency generated by the murine dorsal flexors.

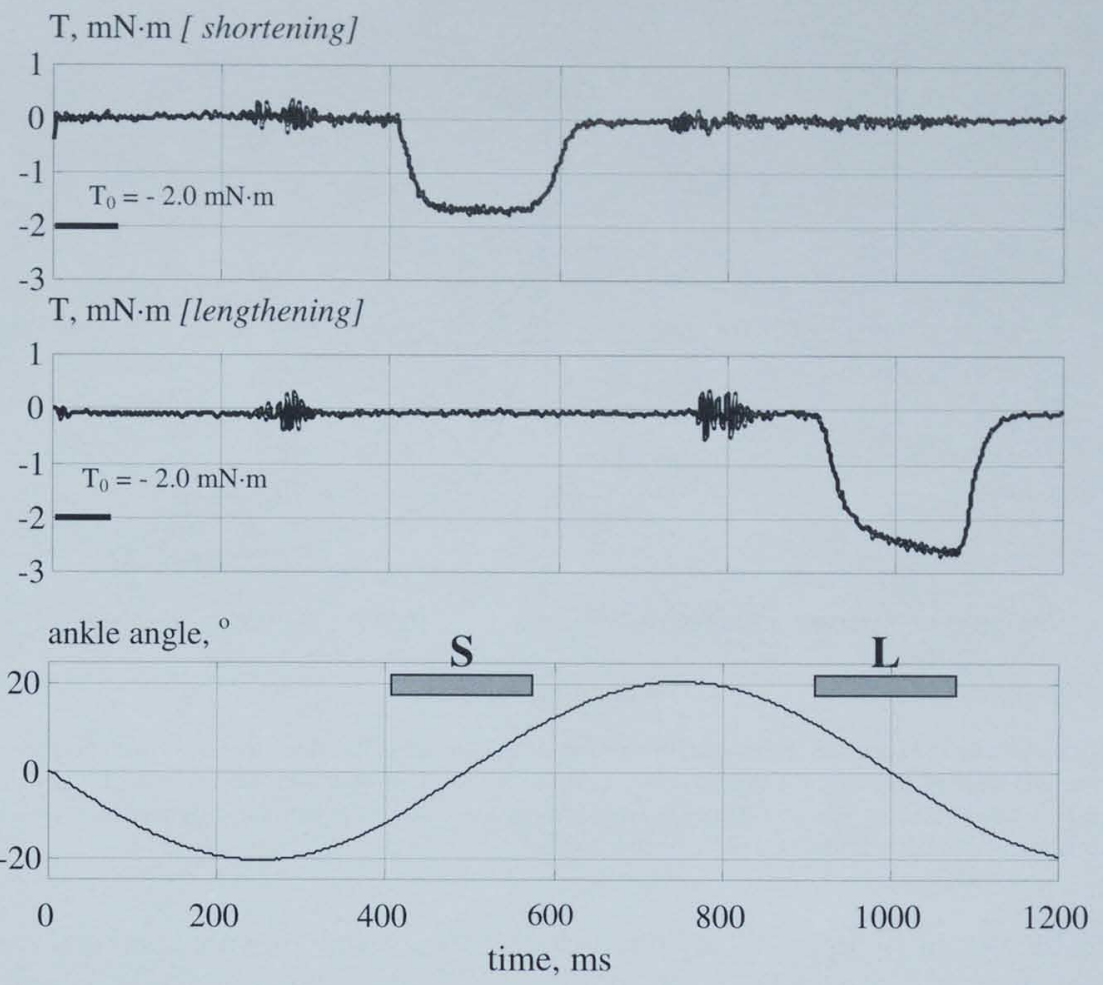

Lower panel shows the ankle angle $\left(^{\circ}\right)$ relative to optimal angle ( $10^{\circ}$ plantar flexion). Negative and positive direction corresponds with plantar and dorsal flexion, respectively. S (shortening) or L (lengthening) indicates the phase of electrical stimulation of the plantar flexors. Maximal isometric torque is indicated by $T_{0}$.

Representative contractile torque-angular velocity relationships for the plantar and dorsal flexor complexes, normalised for maximal torque of an isometric contraction $\left(\mathrm{T}_{\mathrm{o}}\right)$, are depicted in figure 4.8. During lengthening contractions, contractile torque increased significantly above the isometric level, i.e., lengtheningto-isometric torque ratio above an angular velocity of $500^{\circ} \cdot \mathrm{s}^{-1}$ amounted to $1.4 \pm 0.1$ for both the plantar and dorsal flexors. Contractile torque generated during shortening contractions declined progressively with increasing shortening velocity. Peak power amounted to $0.19 \pm 0.03 \mathrm{~W} \cdot \mathrm{g}^{-1}$ and $0.27 \pm 0.02 \mathrm{~W} \cdot \mathrm{g}^{-1}$ for the plantar and dorsal flexors, respectively. 


\section{Chapter 4}

figure 4.8 Normalised torque-velocity relationship of dorsal (o) and plantar (+) flexors.

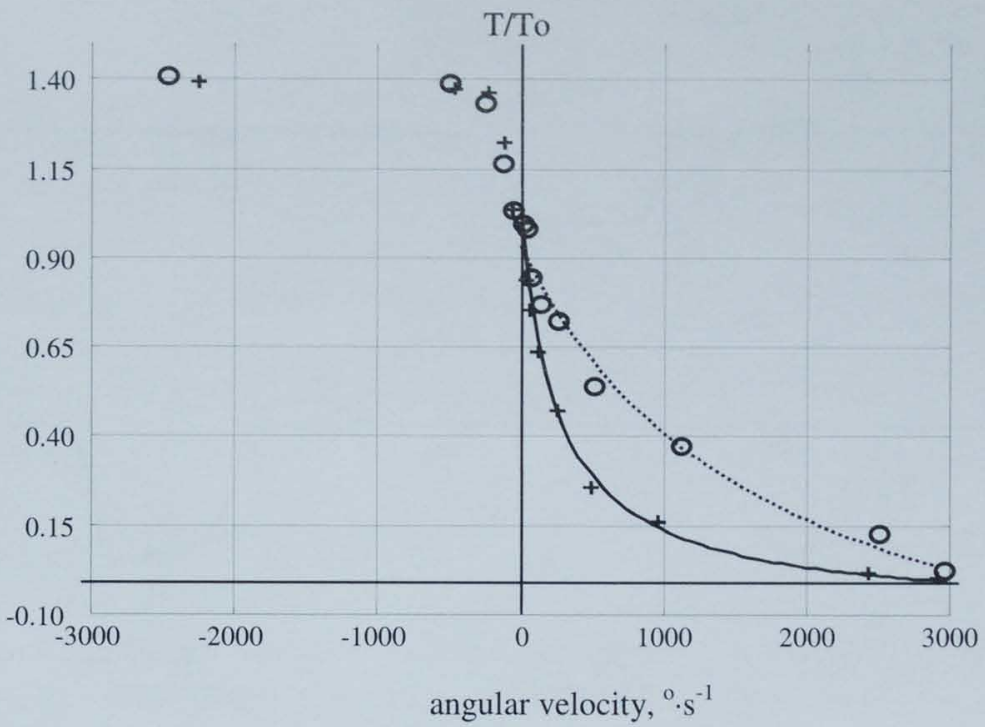

Contraction velocity is expressed as ${ }^{0} \cdot \mathrm{s}^{-1}$ and the torque values are normalised for maximal isometric torque $\left(\mathrm{T}_{0}\right)$. The shortening part of the torque-velocity curve is fitted by the Hill equation, i.e., dorsal (dotted curve) and plantar (solid curve) flexors. Data at zero velocity were assessed with the isometric dynamometer and data at non-zero velocities $\left(12.5\right.$ to $\left.3000^{\circ} \cdot \mathrm{s}^{-1}\right)$ with the ergometer.

The values of optimal angular velocity $\left(\mathrm{V}_{\text {opt }}\right)$ and maximal angular velocity $\left(\mathrm{V}_{\max }\right)$ for both the dorsal and plantar flexors are summarised in table 4.1. Optimal and maximal angular velocities were significantly higher for the dorsal flexor complex than for the plantar flexors.

table 4.1. Characteristics of shortening and lengthening contractions of murine plantar and dorsal flexors.

muscle complex mass, $m g$ isometric torque, $\mathrm{mN} \cdot \mathrm{m}$ maximal lengthening-isometric ratio peak power $\cdot$ muscle mass ${ }^{-1}, W \cdot g^{-1}$

$\mathrm{V}_{\max }, \cdot s^{-1}$

$\mathrm{V}_{\text {opt }}, \cdot s^{-1}$

Hill parameters

$\mathrm{T}_{0}, m N \cdot m$

a, $m N \cdot m$

$\mathrm{a} / \mathrm{T}_{\mathrm{o}}$

$\mathrm{b}$
Plantar flexors

$152.4( \pm 15.7)$
$10.9( \pm 2.3)$
$1.4( \pm 0.1)$
$0.19( \pm 0.03)$
$2580( \pm 180)$
$700( \pm 90)$
$10.6( \pm 2.8)$
$1.9( \pm 1.5)$
$0.2 \pm 0.1$
$7.6( \pm 3.4)$

dorsal flexors

$59.2( \pm 9.3) *$

$2.1( \pm 0.1) *$

$1.4( \pm 0.1)$

$0.27( \pm 0.02) *$

$3420( \pm 380) *$

$1100( \pm 190) *$

$2.1( \pm 0.1) *$

$1.0( \pm 0.4)$

$0.5 \pm 0.2$ *

$33.2( \pm 13.8) *$

Data are expressed as mean $( \pm \mathrm{sd})(\mathrm{n}=4)$. Asterisk indicates significant differences plantar and dorsal flexors. 


\section{5 - Discussion}

An experimental set-up for assessment of shortening and lengthening contraction properties of intact plantar and dorsal flexors of the mouse has been described and evaluated. The main findings of the present study are that the novel ergometer described here provides accurate and valid measurements of contractile properties during shortening and lengthening contractions of the intact murine dorsal and plantar flexor muscles. Evaluation of the fixation procedure via 3-D monitoring of the plantar flexor complex length showed that the additional shortening in length due to a shortening contraction exerted only minor effects on assessed contractile torque. Furthermore, it was found that a realistic misalignment between the ankle axis and measurement shaft (eccentricity) of less than $1.0 \mathrm{~mm}$ did not substantially affect contractile torque.

A pioneering study with respect to analysis of shortening and lengthening muscular contractions of intact mice was performed by Ashton-Miller and co-workers (2). Fixation of the animal in the Ashton-miller set-up was performed via a rigid fixation of the knee and a relative loose fixation of the foot with adhesive tape. As a consequence of inevitable eccentricity, this fixation procedure will most likely result in an additional non-muscular torque. In our set-up, the above mentioned problems are overcome by using a rigid foot fixation and support of the knee in the sagital plane.

\subsubsection{Validation of fixation system}

A potentially confounding factor for in situ assessment of dynamic contractile properties is an altering length of the muscle complex during contraction because of non-rigid fixation of the hindlimb. Maximal alterations in plantar flexor length during a passive stroke amounted to $2.8 \%$ and $3.8 \%$ in the lengthening and shortening phase, respectively. On geometrical basis, one can calculate that the muscle length at $35^{\circ}$ dorsal flexion would be increased by $3.4 \%$, and decreased by $3.7 \%$ at $5^{\circ}$ plantar flexion, assuming a lever arm of $2.1 \mathrm{~mm}$ (2). These values are in accordance with the measured changes in muscle length. So, one could assume that the hindlimb fixation procedure applied in the present study results in reliable changes of muscle length induced during passive strokes. The additional shortening of $0.21 \mathrm{~mm}$ during an active stroke at $1 \mathrm{~Hz}$ oscillation frequency corresponds to approximately $16 \%$ of the total imposed muscle shortening $(1.3 \mathrm{~mm})$. The $16 \%$ extra shortening could affect the muscular torque build up via the force-length and force-velocity relationship. The optimal ankle angle, however, was determined in the contracted condition. So, the change in muscle length during contraction of the plantar flexors, possibly due to nonrigid fixation, has no effect on maximal torque via the force-length relationship. 
The difference in shortening velocity at optimal angle between passive and active oscillations remained below $0.3 \mathrm{~mm} \cdot \mathrm{s}^{-1}$ (fig. 4.3). Since the mean shortening velocity of the muscle equalled $5.2 \mathrm{~mm} \cdot \mathrm{s}^{-1}$, at $1 \mathrm{~Hz}$ and $20^{\circ}$ amplitude, the difference in shortening velocity was $6 \%$. From the assessed Hill parameters (table 4.1 ) it was calculated that the error, due to the difference in shortening velocity, in torque was 3.2 $\%$. During the phase of torque build-up and relaxation, the differences in measured velocity between a passive and an active stroke was maximally $-7.6 \mathrm{~mm} \cdot \mathrm{s}^{-1}$ and 3.0 $\mathrm{mm} \cdot \mathrm{s}^{-1}$, respectively. It is feasible that the build-up and relaxation of the contraction will be affected by this difference in muscle shortening or lengthening velocity. To obtain a reliable estimate of the effect on muscular performance in the build-up and relaxation phase a mathematical model is needed. However, this is beyond the scope of the present study and, hence, temporal parameters, such as the rise time and relaxation time were not calculated.

\subsubsection{Effect of eccentricity on measured torque}

One of the considerations while designing the ergometer was that eccentricity would lead to an additional, non-muscular torque. The non-rigid knee fixation was used to overcome this problem. The maximal torque generated during passive strokes was only affected at eccentricity distances of more than $4.0 \mathrm{~mm}$ in the heel direction and $1.5 \mathrm{~mm}$ in the toe direction (fig. 4.4). Moreover, the effect of eccentricity on the contractile torque at $1 \mathrm{~Hz}$ oscillation frequency was also found to be negligible (fig. 4.5). In contrast, the contractile muscle torque at $8 \mathrm{~Hz}$ oscillation frequency was affected at eccentricity distances exceeding $4.0 \mathrm{~mm}$ in the heel direction and $2.0 \mathrm{~mm}$ in the toe direction. During routine experiments, however, eccentricity will never exceed $1.0 \mathrm{~mm}$. So, the small and inevitable, eccentricity between the ankle joint and measurement axis will not affect the measured contractile properties determined in our experimental set-up.

\subsubsection{Assessment of contractile properties}

Shortening properties of the dorsal and plantar flexors were determined making use of the Hill equation, and were expressed as peak power, and maximal and optimal shortening velocity. Peak power per unit muscle mass of the dorsal flexors was significantly higher than that of the plantar flexors. Moreover, optimal and maximal angular velocities were also significantly higher in the dorsal flexor complex than in the plantar flexor complex (table 4.1). Peak power and shortening velocity of a given muscle complex depend, among others, on its composition of muscle fibre types. This is underscored by data in literature indicating a higher peak power and shortening velocity for type IIB than for IIA fibres (11). 
So, it is most likely that the relatively high proportion of IIB fibres in the dorsal flexors compared to the plantar flexors is one of the causes for the higher peak power per unit muscle mass and higher optimal and shortening velocities in the dorsal flexor complex (12). Brooks and Faulkner (12) estimated the maximum power output of the Extensor Digitorum Longus (EDL) muscle (fast-twitch) at $0.22 \pm 0.02 \mathrm{~W} \cdot \mathrm{g}^{-1}$. Moreover, the curvature of the Hill curve as described with $a / T_{0}$ is comparable to Brooks and Faulkner, i.e. the $\mathrm{a} / \mathrm{P}_{\mathrm{o}}$ for the EDL muscle amounted to 0.4 (15). In this respect, the dorsal and plantar flexor muscles should be compared with the values of the EDL muscle, because they both show a high percentage of fast-twitch fibres (13). The peak power obtained for the EDL muscle are somewhat lower than the values for the dorsal flexors, which could be explained by the properties of the ex vivo experimental set-up used in the latter study (13), which may affect the condition of the muscles, and therefore the power output.

To calculate the muscle complex shortening velocity $\left(\mathrm{L}_{\mathrm{f}} \cdot \mathrm{s}^{-1}\right)$ from the angular velocities, the values of optimal fibre lengths and lever arms must be known. Burkholder and co-workers determined the optimal plantar and dorsal flexor fibre lengths $\left(\mathrm{L}_{\mathrm{f}}\right)$ in Swiss mice at 5.6 and $6.6 \mathrm{~mm}$, respectively (13). Moreover, AshtonMiller and colleagues determined lever arms of the plantar and dorsal flexor complex at $2.1 \mathrm{~mm}$ and $1.0 \mathrm{~mm}$, respectively (2). Using these estimated values of fibre lengths and lever arms, maximal fibre shortening velocity for the plantar and dorsal flexors was calculated at $15.0 \pm 1.0 \mathrm{~L}_{\mathrm{f}} \cdot \mathrm{s}^{-1}$ and $16.8 \pm 1.9 \mathrm{~L}_{\mathrm{f}} \cdot \mathrm{s}^{-1}$, respectively. Furthermore, the optimal fibre shortening velocity was reported at $4.0 \pm 0.5 \mathrm{~L}_{\mathrm{f}} \cdot \mathrm{s}^{-1}$ and $5.4 \pm 0.9 \mathrm{~L}_{\mathrm{f}} \cdot \mathrm{s}^{-1}$ for the plantar and dorsal flexor complexes, respectively. These values are somewhat higher than the values estimated by Brooks and Faulkner for the EDL muscle. They estimated the maximal and optimal shortening velocities at $10.1 \pm 1.2 \mathrm{~L}_{\mathrm{f}} \cdot \mathrm{s}^{-1}$ and $3.3 \pm$ $0.3 \mathrm{~L}_{\mathrm{f}} \cdot \mathrm{s}^{-1}$, respectively (14). The differences between their findings and ours can most likely be explained by the differences in experimental set-up, e.g. in vitro vs. in situ measurements or temperature effects.

\subsubsection{Conclusions}

The present experimental model, i.e., the novel ergometer combined with the isometric dynamometer, is designed for accurate assessment of the in situ muscle complex contraction characteristics of intact dorsal and plantar flexors of anaesthetised mice. The model, including the fixation unit, allows for a reliable and non-invasive appraisal of the shortening and lengthening contractile parameters of these muscle complexes, i.e., maximal peak power, and optimal and maximal shortening velocity. Furthermore, realistic eccentricities of less than $1.0 \mathrm{~mm}$ have only a minor effect on the assessed maximal torque. 
The values of peak power and shortening velocities estimated with the ergometer were higher than the values assessed under in vitro conditions, which can most likely be explained by differences in experimental conditions.

\section{6 - Acknowledgement}

We gratefully acknowledge Prof. Dr. J.D. Janssen from Biomedical Engineering, Eindhoven University of Technology, the Netherlands for his support and helpful advice and the technical support of J.H.M. Wijtvliet of the Eindhoven University of Technology, The Netherlands. 


\section{7 - References}

1. Gorselink, M., Drost, M. R., Louw de, J., Willems, P. J. B., Rosielle, N., Janssen, J. D., and Van der Vusse, G. J. (2000) Accurate assessment of in situ isometric contractile properties of hind limb plantar and dorsal flexor muscle complex of intact mice. Pflügers Arch. 439, 665-670

2. Ashton-Miller, J. A., Veerichetty, Y. H. E., and Kadhiresan, A. (1992) an apparatus to measure in vivo biomechanical behavior of dorsi- and plantarflexors of mouse ankle. Pflugers Arch 72(3), 1205-1211

3. James, R. S., Altringham, J. D., and Goldspink, D. F. (1995) The mechanical properties of fast and slow skeletal muscles of the mouse in relation to their locomotory function. J. Exp. Biol. 198, 491-502

4. Nicolopoulos, S., and Iles, J. F. (1984) Hindlimb muscle activity during locomotion in the rat. J. Zool. London 203, 427-440

5. Hennig, R., and Lomo, T. (1985) Firing patterns of normal units in normal rats. nature 314, 164-166

6. Barratta, R. V., Solomonov, M., Best, R., Zembo, M., and Ambrosia, R. (1995) Forcevelocity relations of nine load-moving skeletal muscles. Med Biol Eng Comput 33, 537-544

7. Christensen, L. V. (1986) Physiology and pathophysiology of skeletal muscle contractions. Part I. Dynamic activity. J.Oral Rehabil. 13, 451-461

8. Wilkie, D. R. (1950) The relation between force and velocity in human muscle. $J$. Physiol. 110, 249-280

9. Van Donkelaar, C. C., Willems, P. J. B., Muijtjens, A. M. M., and Drost, M. R. (1999) Skeletal muscle transverse strain during isometric contraction at different lengths. $J$. Biomech. 32, 755-762

10. Hill, A. V. (1938) The heat of shortening and the dynamic constants of muscle. Proc. Roy. Soc. London B. 126, 136-195

11. Bottinelli, R., Schiaffino, S., and Reggiani, C. (1991) Force-velocity relations and myosin heavy chain isoform compositions of skinned fibres from rat skeletal muscle. J. Physiol. (London) 437, 655-672

12. Brooks, S. V., and Faulkner, J. A. (1991) Forces and powers of slow and fast skeletal muscles in mice during repeated contractions. J. Physiol. 436, 701-710

13. Burkholder, T. J., Fingado, B., Baron, S., and Lieber, R. L. (1994) Relationship between muscle fiber types and sizes and muscle architectural properties in the mouse hindlimb. J. Morphol. 221, 177-190

14. Brooks, S. V., Faulkner, J. A., and McCubbrey, D. A. (1990) Power outputs of slow and fast skeletal muscles of mice. J. Appl. Physiol. 68, 1282-1285

15. Brooks, S.V., Faulkner, J.A. (1988) Contractile properties of skeletal muscles from young, adult and aged mice. J. Physiol. 404, 71-82 
Chapter 4 


\section{Chapter 5}

1 MPAIRED MUSCULAR CONTRACTILE PERFORMANCE AND ADENINE NUCLEOTIDE HANDLING IN CREATINE KINASE DEFICIENT MICE.

M. Gorselink, M.R. Drost, Coumans, W.A., Kranenburg, G.P.J. van, Hesselink, R.P., Vusse, G.J. van der. (2001) Am. J. Physiol, in press

Description of the effect of creatine kinase deficiency on isometric performance in the dorsal flexor muscle complex. 


\section{1 - Summary}

Creatine kinase (CK) forms a small family of iso-enzymes playing an important role in maintaining the concentration of ATP and ADP in muscle cells. To delineate the impact of lack of $\mathrm{CK}$ activity, we studied contractile performance during a single maximal tetanic contraction and during 12 repeated tetanic contractions of intact dorsal flexors of CK knockout mice. To investigate the effect on ATP regeneration, muscular high-energy phosphate content was determined at rest, immediately after the contraction series and after a 60 seconds recovery period.

Maximal torque of the dorsal flexors was significantly lower in $\mathrm{CK}^{-/}$mice than in wild type animals, i.e., $23.7 \pm 5.1$ and $33.3 \pm 6.8 \mathrm{mN} \cdot \mathrm{m} \cdot \mathrm{g}^{-1}$ wet weight, respectively. Lower muscle ATP $\left(20.1 \pm 1.4\right.$ in $\mathrm{CK}^{-1-}$ vs. $28.0 \pm 2.1 \mu \mathrm{mol} \cdot \mathrm{g}^{-1}$ dry weight in controls $)$ and higher IMP ( $1.2 \pm 0.5$ in $\mathrm{CK}^{-/-}$vs. $0.3 \pm 0.1 \mu \mathrm{mol} \cdot \mathrm{g}^{-1}$ dry weight in controls) levels, at the onset of contraction, may contribute to the declined contractility in $\mathrm{CK}^{-1}$ mice. In contrast to wild type muscles, ATP levels could not be maintained during the series of 12 tetanic contractions of dorsal flexors of $\mathrm{CK}^{-/-}$mice and dropped to $15.5 \pm 2.4$ $\mu \mathrm{mol} \cdot \mathrm{g}^{-1}$ dry weight. The significant increase in tissue IMP content $\left(2.4 \pm 1.1 \mu \mathrm{mol} \cdot \mathrm{g}^{-1}\right.$ dry weight) following the contraction series indicates ATP regeneration through adenylate kinase, which activity is inherently not capable to fully compensate for the lack of CK. ATP regeneration via the adenylate kinase pathway is a likely cause of reduced basal adenine nucleotide levels in $\mathrm{CK}^{-/}$mice. 


\section{2 - Introduction}

Creatine kinase (CK) consists of a small family of iso-enzymes catalysing the transphosphorylation between phosphocreatine (PCr) and ATP $(1,2)$. Skeletal muscle contains two distinct isoforms, which are localised either in the cytosol (MM-CK) or at the interior site of the outer mitochondrial membrane $\left(\mathrm{Mi}_{\mathrm{b}}-\mathrm{CK}\right)$ (1-4). Both $\mathrm{CK}$ isoforms play an important role in maintaining muscular ATP and ADP concentrations, e.g. during strenuous exercise (5). Previously, it has been shown that ATP levels are lower in resting skeletal muscle of mice with combined deficiency in $\mathrm{MM}-\mathrm{CK}$ and $\mathrm{Mi}_{\mathrm{b}}-\mathrm{CK}$ (6). Interestingly, the resting skeletal muscle $\mathrm{PCr}$ and creatine content was found to be comparable with that of age-matched control mice, despite the virtual lack of creatine kinase activity in the former $(6,7)$. Moreover, the resting inosine monophosphate (IMP) level was enhanced in CK deficient mice, which strongly suggests a disturbance in ATP regeneration (6).

Skeletal muscle of MM-CK and $\mathrm{Mi}_{b}-\mathrm{CK}$ deficient mice shows impaired contractile properties, which is marked by diminished tetanic force output and prolonged relaxation time $(6,8)$. The relationship between depressed contractility and utilisation of high-energy phosphates in CK deficient mice, however, has been hardly investigated. In the present study, we explored the hypothesis that muscles lacking both creatine kinase isoforms are unable to properly handle their high-energy phosphate stores, which results in an impaired muscular functioning. To test this hypothesis contractile performance during a single tetanic contraction of dorsal flexors of $\mathrm{CK}^{-1-}$ mice was determined, while keeping the muscle in its natural surrounding. Possible differences in contractile behaviour during a single tetanic contraction between $\mathrm{CK}^{-/}$and wild type mice were related to differences in resting content of high-energy phosphates and associated compounds in the muscle group studied. Furthermore, the rate of decline in maximal torque during a series of 12 repetitive isometric tetanic contractions was monitored. By analysing the tissue content of highenergy phosphates and related compounds in resting muscle, immediately after the series of tetanic contractions and following a recovery period of $60 \mathrm{~s}$, we investigated the effect of lack of CK activity on ATP regeneration in muscles subjected to this high-intensity exercise series.

Traditionally, mechanical functioning of muscles of small rodents is determined in less physiological in vitro conditions, e.g. isolated muscles with impaired innervation and blood supply, which may influence the mechanical properties of the muscle under investigation. To study muscle performance under physiological conditions, we recently developed a sensitive and accurate mouse isometric dynamometer (6). 
This approach enabled us to assess maximal tetanic torque and temporal parameters, such as rise time and relaxation time, of intact muscles in anaesthetised mice.

\section{3 - Methods}

\subsubsection{Animals}

Mice lacking both mitochondrial and cytosolic $\mathrm{CK}\left(\mathrm{Mi}_{\mathrm{b}}-\mathrm{CK}^{-/-}\right.$x MM-CK${ }^{-1}$, denoted as $\mathrm{CK}^{-/-}$) (9) were used in the present study. 8 Weeks old male wild type littermates (C57/B16 x 129/sv) served as controls. Halothane (Fluothane ${ }^{\circledR}$, Zeneca, Ridderkerk, The Netherlands) was used as anaesthesia agent, supplied in $\mathrm{O}_{2}$ and $\mathrm{N}_{2} \mathrm{O}$ $(3: 1,1.5-2.0 \%)$ via a facemask through a flowmeter system (4.0 l. $\mathrm{min}^{-1}$; Medec, Montvalle, N.J., USA). All experimental procedures were approved by the Institutional Animal Care and Use Committee of the Maastricht University and complied with the principles of laboratory animal care.

\subsubsection{Surgical procedure}

Mice were positioned on a thermostatic platform $\left(38.5 \pm 0.1{ }^{\circ} \mathrm{C}\right)$. A small incision in the lateral part of the knee was made to expose the peroneal nerve. After locally depilating the skin, a bipolar platinum hook electrode, interpole distance of 0.8 $\mathrm{mm}$, was carefully attached to the nerve and the dorsal flexor muscles complex, consisting of the tibialis anterior and extensor digitorum muscle, was stimulated via a pulse generator (HSE 215/IZ, Freiburg, Germany). The position of the electrode was changed, if a current of more than $1.0 \mathrm{~mA}$ was needed to obtain maximal tetanic muscle contraction. The electrode was fixated to the skin with cyano-acrylate glue to prevent electrode displacement during muscle contraction.

\subsubsection{Experimental measurement set-up}

For mechanical analysis, the anaesthetised mouse was fixated via the hip and foot to the measurement device. Dorsal flexor torque around the ankle joint was measured with a sensitive and accurate custom-built device, the details of which were published earlier (10). Data acquisition was performed at $1000 \mathrm{~Hz}$ with an Apple Macintosh 7100 PowerPC $^{\circledR}$ with an 8 channel, 12 bits Lab-NB analogue-to-digital conversion board (National Instruments ${ }^{\circledR}$, Mopac Expwy, Austin, Tx, USA). Postprocessing of the torque data was performed with Matlab 5.2.1 (The Math Works, Inc., Natick, Ma, USA). 


\subsubsection{Exercise protocol}

Supra-maximal stimulation current, necessary to obtain recruitment of all muscle fibres, was first determined using 3 to 5 isometric twitch contractions with increasing current. Resting periods between twitches were kept constant at $60 \mathrm{~s}$. Optimal muscle length, at optimal ankle angle was determined using 11 twitch contractions at ankle angles between $20^{\circ}$ dorsal flexion and $30^{\circ}$ plantar flexion. The ankle angle at which the muscle exerted a maximal torque was not different between the wild type and $\mathrm{CK}^{-1-}$ mice, and amounted to $19.1 \pm 5.9$ and $17.6 \pm 4.0^{\circ}$, respectively. Maximal tetanic torque was obtained with a $200 \mathrm{~ms}$ pulse train and 125 $\mathrm{Hz}$ stimulation frequency. Subsequently, dorsal flexors were subjected to 12 isometric tetanic contractions through electrical stimulation of the peroneal nerve for $170 \mathrm{~ms}$ every $265 \mathrm{~ms}$ (tour duty cycle: 0.64), at optimal ankle angle, $125 \mathrm{~Hz}, 0.5 \mathrm{~ms}$ pulse duration, and a supra-maximal stimulation current.

Torque signals $(\mathrm{n}=8)$ were digitised and analysed for maximal tetanic torque, rise time, and $1 / 2$-relaxation time. Maximal torque was determined from filtered torque signals during electrical stimulation (11-point moving average). Absolute tetanic torque values were normalised for the wet muscle complex mass $\left(\mathrm{mN} \cdot \mathrm{m} \cdot \mathrm{g}^{-1}\right.$ muscle mass). Both the rise time and the $1 / 2$-relaxation time were determined from the unfiltered torque signals. The rise time is defined as the time required increasing the muscle torque from 10 to $50 \%$ of the maximal torque. The $1 / 2$-relaxation time is the time to decrease the torque from 50 to $25 \%$ of the maximal torque after cessation of electrical stimulation (11). Furthermore, the maximal torque value of each single contraction during the series of 12 contractions was determined. Temporal properties were characterised in terms of $1 / 2$ - relaxation time of the $12^{\text {th }}$ contraction and the rise time of the consecutive contractions.

\subsubsection{Tissue sampling}

Subsets of mice were used for muscular tissue sampling at two different timepoints during the contraction protocol for the analysis of tissue high-energy phosphates and related compounds, i.e., immediately after the series of 12 contractions $(E, n=6)$, and after $60 \mathrm{~s}$ recovery following $E(E+R, n=6)$. Control samples $(C, n=8)$ were taken from the resting, contra-lateral dorsal flexor complex. Muscular tissue was rapidly freeze-clamped using a pair of aluminium tongs cooled in liquid nitrogen. The tissue samples were stored at $-80{ }^{\circ} \mathrm{C}$ until analysis. For tissue sampling immediately after the contraction series (E), a slightly different experimental set-up was used to prevent damage of the sensitive torque-measuring device. First, the optimal stimulation conditions, i.e., ankle angle and supra-maximal stimulation current, were determined with the isometric mouse dynamometer. 
Thereafter, this device was replaced by a dummy apparatus, keeping the ankle angle, and therefore the muscle length, at optimal value. The same stimulation protocol was applied to the muscles. The time interval between the final contraction and the freezing procedure was kept as short as possible $(<2 \mathrm{~s})$.

Prior to high-energy phosphate analysis, the muscle samples were freeze-dried overnight at $-30^{\circ} \mathrm{C}$. High-energy phosphates and related compounds were assessed with HPLC as described by Wijnants and Van Belle (12) and modified by Van der Vusse and co-workers (13). Total adenine nucleotide content (TAN) was computed as the sum of ATP, ADP, and AMP. One set of contra-lateral dorsal flexor muscles from wild type and $\mathrm{CK}^{-/}$mice was used for determination of maximal activity of creatine kinase, adenylate kinase and AMP deaminase. Briefly, muscle complexes were homogenised in SET buffer (0.25 M sucrose, 0.002 M EDTA, $0.01 \mathrm{M}$ Tris at $\mathrm{pH}$ 7.4) and stored at $-80{ }^{\circ} \mathrm{C}$. Maximal activity of creatine kinase (EC 2.7.3.2) and adenylate kinase (EC 2.7.4.3) was determined using the coupled enzyme assay of hexokinase (EC 2.7.1.1) and glucose-6-phosphate dehydrogenase (EC 1.1.1.49) producing NADPH. AMP deaminase (EC 3.5.4.6) activity was assessed according to Rundell and co-workers (14). Maximal enzyme activities were measured with a centrifugal analyser (Cobas Fara, La-Roche, Switzerland) and expressed as $\mu \mathrm{mol} \cdot \mathrm{min}^{-1} \cdot \mathrm{g}^{-1}$ muscular wet weight.

\subsubsection{Number of animals and statistical procedures}

In total $14 \mathrm{CK}^{-1-}$ and 14 wild type littermates were included in this study. Mechanical activity was measured in $8 \mathrm{CK}^{-1}$ and 8 wild type animals. The dorsal flexor muscle complex was freeze-clamped $60 \mathrm{~s}$ after the high-intensity contraction series. Due to technical imperfections only in 6 out of 8 muscle complexes the highenergy phosphate content was assessed. In a subset of 6 animals the muscle complex was freeze-clamped immediately after he high-intensity contraction series, and used for HPLC analysis (see above). The contra-lateral, resting muscle complex was freezeclamped in $14 \mathrm{CK}^{-1-}$ and WT animals, respectively. Eight and 5 tissue samples of each group were used for HPLC analysis and assessment of enzymatic activity, respectively.

Data are expressed as mean \pm standard deviation. Differences in mechanical performance during a single tetanic contraction were analysed with a Mann-Whitney $\mathrm{U}$ test. Variation in mechanical performance and in high-energy phosphates following the contraction series between CK deficient and wild type mice was analysed using a one-way ANOVA. Tukey post-hoc analyses were performed to identify differences in mechanical output and high-energy phosphate values between basal level (C) and the time periods $\mathrm{E}$ and $\mathrm{E}+\mathrm{R}$ of the repetitive contraction protocol. 
The differences were considered significant if $\mathrm{p}<0.05$. SPSS 9.0 (SPSS Benelux, Gorinchem, The Netherlands) was used for statistical analyses.

\section{4 - Results}

Mean body mass of $\mathrm{CK}^{-/-}$mice $(21.9 \pm 1.7 \mathrm{~g})$ was significantly lower than wild type body mass $(24.0 \pm 1.0 \mathrm{~g})$. Dorsal flexor mass of $\mathrm{CK}^{-1-}$ mice amounted to $52.5 \pm$ $6.1 \mathrm{mg}$, and was significantly lower than that of the age-matched wild type mice $(62.1$ $\pm 8.2 \mathrm{mg})$.

figure 5.1 Representative pattern of developed torque during a single isometric tetanic contraction of the dorsal flexor muscle of wild type and $\mathrm{CK}^{-1-}$ mice.

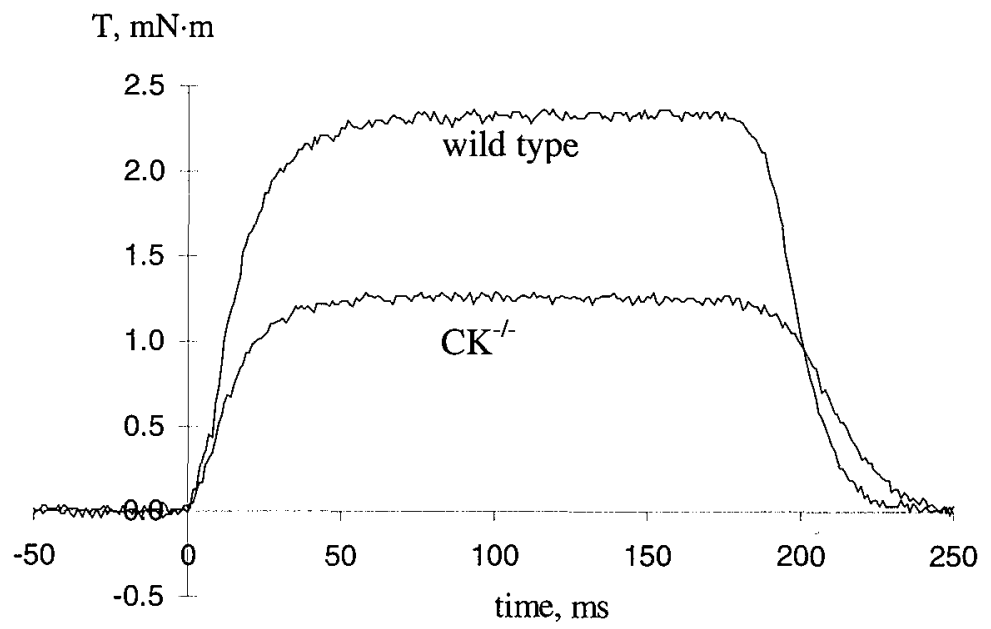

Dorsal flexors were stimulated with $125 \mathrm{~Hz}, 0.5 \mathrm{~ms}$, and a supra-maximal stimulation current. Electrical stimulation started at $0 \mathrm{~ms}$ and was switched off at $170 \mathrm{~ms}$.

Figure 5.1 shows a representative maximal dorsal flexor tetanic torque pattern for wild type and $\mathrm{CK}^{-1-}$ mice at $125 \mathrm{~Hz}$ stimulation frequency. The mean values of isometric contractile parameters of dorsal flexors measured in $\mathrm{CK}^{-/}$and wild type mice are shown in table 5.1. Maximal tetanic torque at a stimulation frequency at $125 \mathrm{~Hz}$ was significantly higher in wild type mice compared to $\mathrm{CK}^{-/}$mice, which could be explained partially by the decline in muscle mass in $\mathrm{CK}^{-1}$ mice. However, after normalisation of the values of the maximal tetanic torque on muscle mass, maximal tetanic torque was still significantly lower in $\mathrm{CK}^{-1}$. The rate of relaxation after tetanic contraction was significantly slower in $\mathrm{CK}^{--}$than control mice, whereas the rise time was not affected in $\mathrm{CK}^{-1-}$ dorsal flexors (table 5.1). 
table 5.1 Torque characteristics of a single tetanic contraction of dorsal flexor muscle of wild type (WT) and $\mathrm{CK}^{-1}$ mice.

\begin{tabular}{lll}
\hline \hline & WT & CK $^{-*}$ \\
\cline { 2 - 3 } & & \\
tetanic torque, $m N \cdot m$ & $2.09 \pm 0.25$ & $1.19 \pm 0.16^{* *}$ \\
mass normalised tetanic torque, $m N \cdot m \cdot g^{-1}$ wet weight & $33.3 \pm 6.8$ & $23.7 \pm 5.1^{*}$ \\
rise time, $m s$ & $11.2 \pm 1.3$ & $11.0 \pm 1.4$ \\
1/2-relaxation time, $m s$ & $11.5 \pm 2.8$ & $16.3 \pm 3.0 *$ \\
\hline
\end{tabular}

Dorsal flexors ( $\mathrm{n}=8$ in each group) were stimulated with $125 \mathrm{~Hz}, 0.5 \mathrm{~ms}$ and a supra-maximal stimulation current. Total duration of the tetanic contraction amounted to $170 \mathrm{~ms}$. Statistical differences between wild type and $\mathrm{CK}^{-1}$ are indicated by $*(\mathrm{p}<0.05)$ or $* *(\mathrm{p}<0.01)$.

figure 5.2 Representative dorsal flexor torque output during a series of 12 tetanic isometric contractions in wild type and $\mathrm{CK}^{-1-}$ mice.

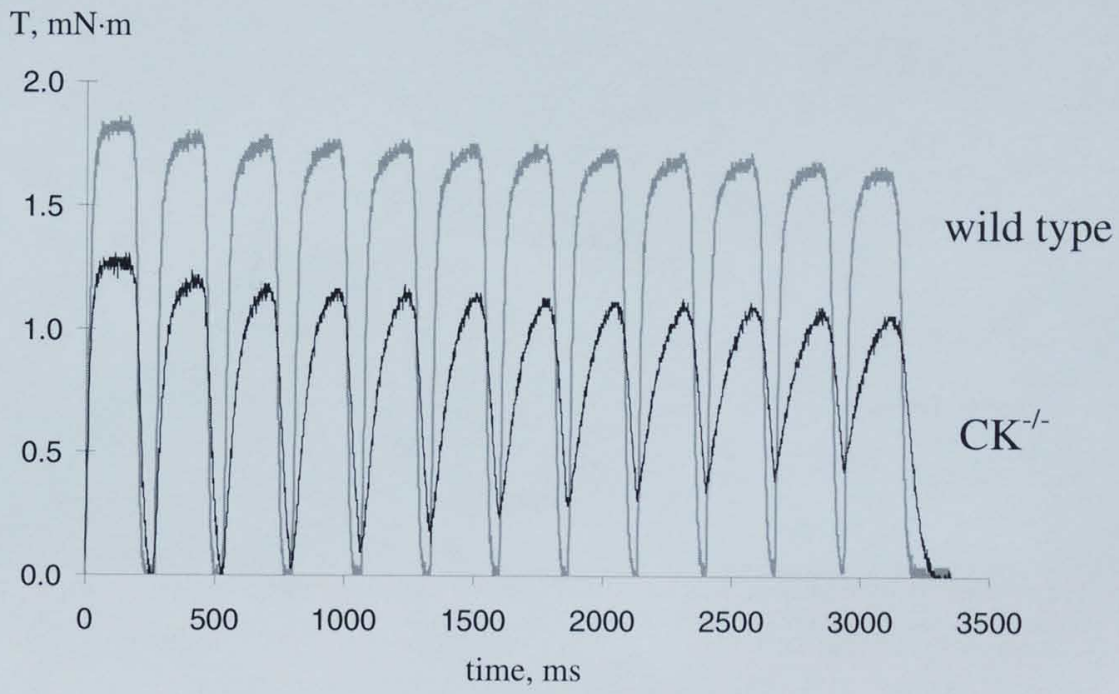

Muscles were stimulated every $265 \mathrm{~ms}$ for $170 \mathrm{~ms}$ with $125 \mathrm{~Hz}, 0.5 \mathrm{~ms}$, and a supra-maximal stimulation current.

A representative torque output signal of a series of 12 consecutive tetanic contractions of dorsal flexors of wild type and $\mathrm{CK}^{-1-}$ mice is given in figure 5.2. In both cases, the maximal torque declined during the contraction series. Figure 5.2 also shows that the rates of torque build up and relaxation are prolonged in $\mathrm{CK}^{-1-}$ mice during the course of the 12 consecutive contractions.

The mean maximal torque of each individual contraction during the series of 12 tetanic concentrations is depicted in figure 5.3. The maximal torque of both the wild type and $\mathrm{CK}^{-/-}$mice showed an approximately linear decrease. 
The maximal torque of the $12^{\text {th }}$ contraction was $12.0 \pm 3.3 \%$ lower than that of the first contraction of wild type mice. The maximal torque output of $\mathrm{CK}^{-1-}$ mice tended to show a larger decrease at the last contraction $(22.9 \pm 9.8 \%)$, but due to the relatively high inter-individual variation this value was not significantly different from the corresponding value of wild type.

figure 5.3 Dorsal flexor torque per unit mass output during 12 tetanic isometric contractions in wild type mice ( $\mathbf{-})$ and $\mathrm{CK}^{-1}$ mice $(\mathbf{\Delta})$.

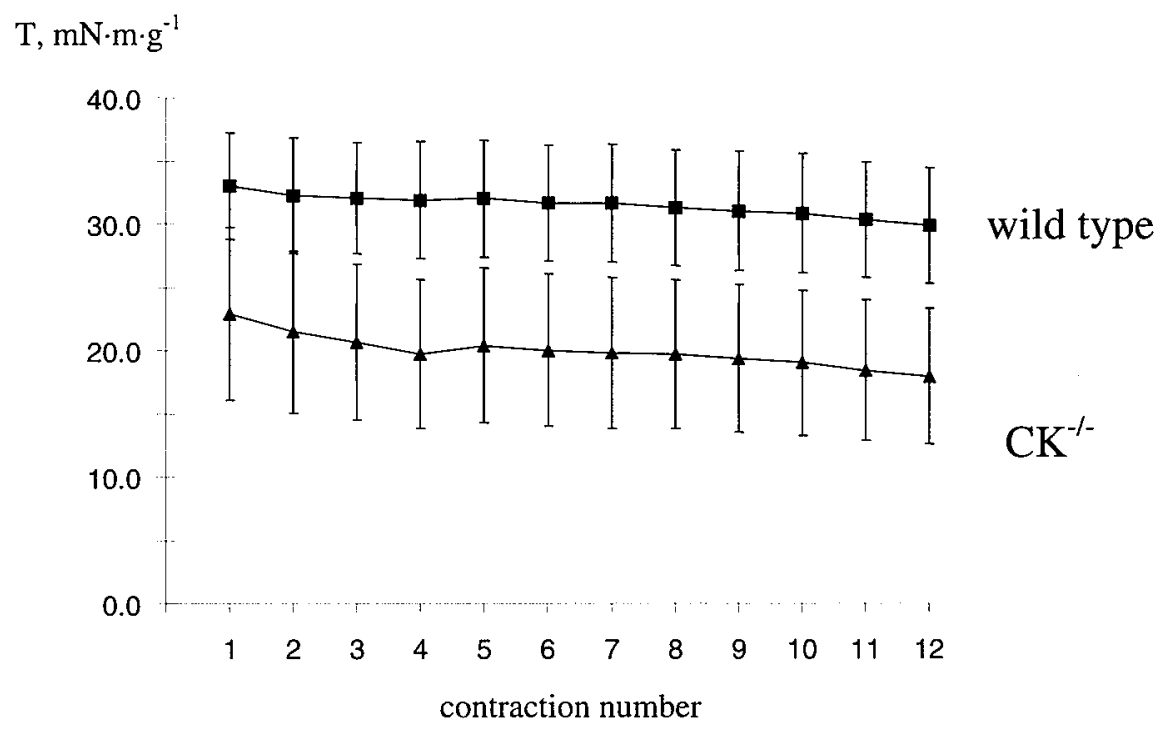

Data and vertical lines shown in figure refer to mean and SD, respectively ( $n=8$ in each group).

The higher relaxation time, as observed in a single tetanic contraction, was even more conspicuous in the series of 12 tetanic contractions. The 1/2-relaxation time at the $12^{\text {th }}$ contraction of $\mathrm{CK}^{-1-}$ mice was significantly longer compared to wild type mice, i.e., $26.2 \pm 2.8 \mathrm{~ms}$ vs. $12.9 \pm 3.5 \mathrm{~ms}$. Furthermore, in $\mathrm{CK}^{-1-}$ mice the $1 / 2$-relaxation time of the $12^{\text {th }}$ contraction was significantly longer than the $1 / 2$-relaxation time measured after a single tetanic contraction; this phenomenon was not present in experiments of wild type mice. Moreover, the rise time of the first contraction was comparable to the values calculated during a single tetanic contraction, i.e., $11.1 \pm 1.2 \mathrm{~ms}$ and $11.0 \pm 1.4$ $\mathrm{ms}$ for wild type and $\mathrm{CK}^{-/-}$mice, respectively. Wild type muscles showed no difference in rise time during the 12 consecutive contractions compared to the initial rise time, in contrast to the $\mathrm{CK}^{-1-}$, which showed a substantial increase in rise time in the second and third contraction, i.e., $19.6 \pm 3.0 \mathrm{~ms}$ and $29.4 \pm 3.7 \mathrm{~ms}$, respectively. 
The rise time of the $4^{\text {th }}$ and following contractions could not be calculated, because the relaxation was prolonged to such extent that torque did not fell below $10 \%$ of the maximal torque value.

Table 5.2 shows the high-energy phosphate content of dorsal flexors at three different time points of the repeated contraction protocol for both wild type and $\mathrm{CK}^{-/}$ mice.

table 5.2 The content of high-energy phosphates and related compounds in dorsal flexors of wild type (WT) and $\mathrm{CK}^{-1-}$ mice.

\begin{tabular}{|c|c|c|c|c|c|c|}
\hline & \multicolumn{2}{|c|}{ C } & \multicolumn{2}{|c|}{$\mathbf{E}$} & \multicolumn{2}{|c|}{$\mathbf{E}+\mathbf{R}$} \\
\hline & $\begin{array}{c}\text { WT } \\
(n=8)\end{array}$ & $\begin{array}{c}\mathrm{CK}^{-/-} \\
(n=8)\end{array}$ & $\begin{array}{c}\text { WT } \\
(n=6)\end{array}$ & $\begin{array}{l}\mathrm{CK}^{-1-} \\
(n=6)\end{array}$ & $\begin{array}{c}\text { WT } \\
(n=6)\end{array}$ & $\begin{array}{l}\mathrm{CK}^{-1-} \\
(n=6)\end{array}$ \\
\hline ATP & $28.0 \pm 2.1$ & $20.1 \pm 1.4^{* *}$ & $27.9 \pm 2.0$ & $15.5 \pm 2.4 \#$ & $27.9 \pm 2.2$ & $14.4 \pm 3.6 \#$ \\
\hline ADP & $4.0 \pm 0.5$ & $5.6 \pm 1.2 *$ & $3.7 \pm 0.6$ & $4.8 \pm 1.3$ & $3.8 \pm 0.4$ & $3.6 \pm 1.0 \#$ \\
\hline AMP & $0.2 \pm 0.1$ & $0.6 \pm 0.2 *$ & $0.2 \pm 0.1$ & $0.4 \pm 0.2$ & $0.2 \pm 0.1$ & $0.3 \pm 0.0 \#$ \\
\hline TAN & $32.2 \pm 2.3$ & $26.3 \pm 1.7 *$ & $31.9 \pm 2.5$ & $20.7 \pm 3.8 \#$ & $32.0 \pm 2.6$ & $18.3 \pm 4.1 \#$ \\
\hline IMP & $0.3 \pm 0.1$ & $1.2 \pm 0.5 *$ & $0.2 \pm 0.1$ & $2.4 \pm 1.1 \#$ & $0.2 \pm 0.1$ & $1.8 \pm 1.1$ \\
\hline $\mathrm{PCr}$ & $73.7 \pm 7.5$ & $69.3 \pm 6.6$ & $57.0 \pm 4.7 \#$ & $65.0 \pm 2.1$ & $80.9 \pm 8.3$ & $66.1 \pm 9.0$ \\
\hline $\mathrm{Cr}$ & $46.3 \pm 5.8$ & $42.6 \pm 5.2$ & $61.2 \pm 12.6 \#$ & $37.2 \pm 2.8$ & $38.7 \pm 3.6$ & $39.6 \pm 3.5$ \\
\hline $\mathrm{PCr}+\mathrm{Cr}$ & $120.0 \pm 8.3$ & $111.9 \pm 11.4$ & $116.2 \pm 13.8$ & $102.2 \pm 4.8$ & $119.7 \pm 8.6$ & $105.7 \pm 12.1$ \\
\hline $\mathrm{PCr} / \mathrm{Cr}$ & $1.6 \pm 0.3$ & $1.6 \pm 0.1$ & $0.9 \pm 0.2 \#$ & $1.8 \pm 0.1$ & $2.1 \pm 0.3 \#$ & $1.7 \pm 0.2$ \\
\hline
\end{tabular}

$\mathrm{C}$ refers to resting values; $\mathrm{E}$ to the content measured in tissue freeze-clamped immediately after a series of 12 tetanic contractions; $\mathrm{E}+\mathrm{R}$ to the content measured in tissue freeze-clamped at the end of a $60 \mathrm{~s}$ recovery period following a series of 12 tetanic contractions. Data refer to mean \pm SD and are expressed as $\mu \mathrm{mol} \cdot \mathrm{g}^{-1}$ dry weight. The ratio phosphocreatine $(\mathrm{PCr}) / \mathrm{creatine}(\mathrm{Cr})$ is dimensionless. Statistical differences between wild type and $\mathrm{CK}^{-1-}$ mice in resting conditions are indicated by $*(\mathrm{p}<0.05)$ and $* *(\mathrm{p}<0.01)$. Statistical differences between either $\mathrm{E}$ or $\mathrm{E}+\mathrm{R}$ with $\mathrm{C}$ are indicated by \# $(\mathrm{p}<0.05)$.

control $(\mathrm{C})$ : Basal phosphocreatine $(\mathrm{PCr})$ and creatine $(\mathrm{Cr})$ levels were unaffected in $\mathrm{CK}^{-/}$muscles, which resulted in comparable $\mathrm{PCr} / \mathrm{Cr}$ ratios between $\mathrm{CK}^{-/}$and wild type muscles. Resting ATP values of $\mathrm{CK}^{-1-}$ mice were significantly lower than wild type, whereas the ADP and AMP content was significantly higher in $\mathrm{CK}^{-1-}$ mice. The sum of adenine nucleotides (TAN) was significantly lower in $\mathrm{CK}^{-1-}$ dorsal flexors compared to wild type mice. Furthermore, resting IMP levels showed a significant fourfold increase in $\mathrm{CK}^{-/-}$mice compared to wild type control muscles.

At the end of contraction $(E)$ : Immediately after the series of 12 consecutive tetanic contractions, the PCr level in wild type mice was significantly lower $(\Delta \mathrm{PCr}=-16.7$ $\left.\mu \mathrm{mol} \cdot \mathrm{g}^{-1} \mathrm{~d} . \mathrm{w}.\right)$, whereas the $\mathrm{Cr}$ level increased to a comparable extent $(\Delta \mathrm{Cr}=14.9$ $\mu \mathrm{mol} \cdot \mathrm{g}^{-1} \mathrm{~d}$.w.). $\mathrm{PCr} / \mathrm{Cr}$ ratio of wild type mice dropped from $1.6 \pm 0.3$ in resting conditions to $0.9 \pm 0.2$ after the contraction series. 
In contrast, $\mathrm{PCr}$ and $\mathrm{Cr}$ levels in $\mathrm{CK}^{-1}$ dorsal flexors were not affected during the series of 12 tetanic contractions, which resulted in an unchanged $\mathrm{PCr} / \mathrm{Cr}$ ratio. At the end of the contraction protocol, $\mathrm{CK}^{-1}$ ATP level was significantly lower as compared to the resting values. ADP and AMP levels did not change, whereas TAN significantly decreased. This contraction-induced decline in ATP was absent in wild type mice. Compared to resting values, $\mathrm{CK}^{-1-}$ muscles showed a significant twofold increase in IMP content at the end of the series of tetanic contractions. In contrast, no accumulation of IMP occurred in wild type muscles.

After $60 \mathrm{~s}$ of recovery $(E+R)$ : During the $60 \mathrm{~s}$ recovery period following the series of 12 tetanic contractions, $\mathrm{PCr}$ and $\mathrm{Cr}$ levels and $\mathrm{PCr} / \mathrm{Cr}$ ratio in wild type mice returned to pre-contraction levels. In $\mathrm{CK}^{-1-}$ muscles, recuperation of ATP levels did not occur during the recovery period, which was also the case for TAN. IMP levels in CK mutant mice remained elevated during the $60 \mathrm{~s}$ recovery period.

table 5.3 Maximal activity of total creatine kinase, adenylate kinase and AMP deaminase in dorsal flexor muscles of wild type (WT) and $\mathrm{CK}^{-/}$mice.

\begin{tabular}{lll}
\hline & WT & CK $^{-{ }^{-}}$ \\
\cline { 2 - 3 } & & \\
\cline { 2 - 3 } total creatine kinase, $U \cdot g^{-1}$ wet weight & $5668 \pm 832$ & $37 \pm 10^{* *}$ \\
Adenylate kinase, $U \cdot g^{-1}$ wet weight & $36.9 \pm 7.9$ & $30.7 \pm 3.9$ \\
AMP deaminase, $U \cdot g^{-1}$ wet weight & $51.6 \pm 14.5$ & $48.4 \pm 12.7$ \\
\hline
\end{tabular}

Values refer to mean $\pm \mathrm{SD}\left(\mathrm{n}=5\right.$ for each group). Statistical differences between wild type and $\mathrm{CK}^{-t}$ are indicated by $* *(\mathrm{p}<0.01)$.

The maximal activity of total creatine kinase in $\mathrm{CK}^{-1-}$ muscle was on the order of $0.7 \%$ of that of wild type. No differences between wild type and $\mathrm{CK}^{-/-}$dorsal flexors in maximal activity of adenylate kinase and AMP deaminase were found (table $5.3)$.

\section{5 - Discussion}

Total CK activity in dorsal flexors of the double CK knockout mice was reduced to $\sim 0.7 \%$ of that of wild type animals. The residual activity can most likely be attributed to the $\mathrm{B}-\mathrm{CK}$ isoform present in vascular endothelium and muscle satellite cells. Using nuclear magnetic resonance analysis, Steeghs and co-workers (9) confirmed that $\mathrm{PCr}$ no longer could be used to regenerate hydrolysed ATP in $\mathrm{CK}^{-1-}$ mice. 
Hence, double CK deficient mice are a useful model to study the impact of lack of CK on muscle contractile properties and adenine nucleotide handling during high-intensity contractions.

\subsubsection{Single isometric tetanic contraction}

The present study clearly shows that the maximal tetanic torque output of $\mathrm{CK}^{-/}$ mice is significantly declined. This finding implicates that lack of creatine kinase activity substantially affects contractile function of intact muscles, investigated in their natural environment. Our in situ observation corroborates earlier findings obtained with isolated muscles, showing a decline in force of muscles lacking CK activity $(6,7$, 15). Theoretically, alterations in the maximal value of muscle torque can be caused, among others, by variation in muscle mass, altered length of the muscle lever arm, and/or changes in energy metabolism. We observed a $16 \%$ decline in dorsal flexor muscular mass of the $\mathrm{CK}^{-1}$ mouse. After normalisation of maximal tetanic torque on muscle mass, torque per gram tissue was still $29 \%$ lower in $\mathrm{CK}^{-1}$ mice than that in littermates. It is of interest to note that Steeghs and colleagues (6) previously observed a $30 \%$ decline in maximal force output in isolated gastrocnemius medialis muscle of $\mathrm{CK}^{-/}$mice. These combined findings strongly suggest that alterations in lever arm do not likely occur in CK mutant mice, indicating that additional factors are involved in the decline of maximal torque.

Altered levels of high-energy phosphates and related compounds in skeletal muscle of $\mathrm{CK}^{-1-}$ mice may play a role in the decrease in maximal torque. In the past, a variety of substances has been put forward as likely candidates that can negatively affect muscular contractility, such as raised concentrations of inorganic phosphate $\left(\mathrm{P}_{\mathrm{i}}\right)$ and IMP or low ATP levels $(16,17)$. Differences in tissue $\mathrm{pH}$ should be also taken into consideration. A decisive role of hydrogen ions in depressed muscular function of $\mathrm{CK}^{-1-}$ mouse is less likely. First, Steeghs and co-workers (14) recently showed that $\mathrm{pH}$ both in resting and contracting $\mathrm{CK}^{--}$muscles did not differ from wild type. Second, the production of lactate during contractions of EDL and soleus muscles was even smaller in $\mathrm{CK}^{-1-}$ mice than in corresponding muscles of wild type littermates (4). It is of interest to note that Dahlstedt and co-workers (7) recently reported a twofold increase in resting $\mathrm{P}_{\mathrm{i}}$ levels in EDL muscle of $\mathrm{CK}^{-/-}$mice. Since $\mathrm{P}_{\mathrm{i}}$ is known to affect the strong cross-bridge attachment and myofibrillar $\mathrm{Ca}^{2+}$ sensitivity (18), higher cellular $\mathrm{P}_{\mathrm{i}}$ concentrations may be a causative factor in the decline of maximal torque of the $\mathrm{CK}^{-1}$ muscles. Furthermore, the resting IMP level in the dorsal flexor muscle was found to be increased fourfold in $\mathrm{CK}^{-1-}$ mice (table 5.2), which indicates a change in the ATP regenerating process in muscle cells lacking CK activity (19). 
Although the precise mechanisms of action are unclear (20), the results obtained in in vitro studies suggest that IMP may interfere with $\mathrm{Mg}^{2+}$-myosin ATPase activity (21), hampering the formation of the myosin-actin complex. In this light, the substantially elevated IMP content in the dorsal flexor muscle of $\mathrm{CK}^{-1}$ mice could be considered as a likely candidate involved in the decline in torque output. The significantly lower ATP content in resting muscle of the $\mathrm{CK}^{-/}$mouse may also contribute to the lower torque output. Westerblad and colleagues (17) observed a correlation between reduced muscular ATP levels and rise in intracellular $\mathrm{Ca}^{2+}$, suggesting a possible role of ATP via calcium ions in impaired muscular function of $\mathrm{CK}^{-1-}$ mice. However, Dahlstedt and co-workers (7) recently showed that, using a single fibre preparation, tetanic $\mathrm{Ca}^{2+}$ levels did not differ between $\mathrm{CK}^{-1}$ and control mice. This finding dismisses the possibility that a low cellular ATP level negatively influence torque output via alterations in $\mathrm{Ca}^{2+}$ handling. An alternative mechanism might be a direct effect of lower ATP levels on ATPase activity, and, hence, on the maximal tetanic torque output via reduced cross-bridge cycling (myosin ATPase) and/or impaired $\mathrm{Na}^{+}-\mathrm{K}^{+}$ ATPase pump.

The $1 / 2$-relaxation time of a single maximal tetanic contraction was significantly higher $(\sim 42 \%)$ in $\mathrm{CK}^{-1}$ mice than in wild type. Our findings in intact animals are in line with the earlier observed lengthening of relaxation time of isolated muscles of $\mathrm{CK}^{-1}$ mice (6). The combined results point towards a disturbed re-uptake of $\mathrm{Ca}^{2+}$ after muscle contraction, in which process energy dependent $\mathrm{Ca}^{2+}$-ATPase pumps (SERCA II) in the sarcoplasmatic reticulum (SR) are the key players (22). It should be emphasised, however, that no differences in mRNA and protein content or activity of SERCA were found between $\mathrm{CK}^{--}$and wild type gastrocnemius muscle (6). Therefore, if $\mathrm{Ca}^{2+}$ re-uptake in the $\mathrm{SR}$ is impaired in $\mathrm{CK}^{-1-}$ muscles, this process may be directly affected by alterations in the energy status of the muscle cell, i.e., no temporal buffering of ATP by PCr and, hence, a decline in ATP concentration at the site of action.

\subsubsection{Repetitive tetanic contractions}

When dorsal flexor muscles of $\mathrm{CK}^{-1-}$ and wild type mice are energetically challenged by a series of 12 high-intensity tetanic contractions, tour duty cycle 0.64 , several interesting differences in torque output became apparent. Although the decline in maximal torque during the course of 12 tetanic contractions tended to be greater in $\mathrm{CK}^{-/}$muscles, the difference between $\mathrm{CK}^{-/}$and wild type did not reached the level of significance. The relaxation time significantly increased in $\mathrm{CK}^{-/}$mice during the series of 12 contractions. The rise time became significantly longer in $\mathrm{CK}^{-f-}$ muscles, whereas the rise time in wild type muscles remained constant in the consecutive contractions. 
These phenomena indicate impaired tolerance of muscles, lacking both mitochondrial and cytoplasmic CK, towards high-intensity contractile activity. Since the present experimental approach does not allow elucidating the precise mechanisms underlying the increased intolerance, one may speculate that the lack of buffering of ATP by phosphocreatine attributes to a great extent to the accelerated decline in rate of developed torque and lengthening of relaxation time.

Assuming that in wild type muscle the major mechanism of ATP regeneration during the series of 12 high-intensity contractions is rephosphorylation of ADP by $\mathrm{PCr}$, on the order of $16.7 \mu \mathrm{mol} \sim \mathrm{P} \cdot \mathrm{g}^{-1} \mathrm{~d}$.w. is consumed during the course of the contractile activity. This value is calculated on basis of the mean of the absolute decline in $\mathrm{PCr}$ and absolute increase in $\mathrm{Cr}$ (table 5.2). During the 12 consecutive contractions, total torque time integral amounted $85.7 \pm 12.1 \mathrm{mN} \cdot \mathrm{m} \cdot \mathrm{s} \cdot \mathrm{g}^{-1}$ and $58.9 \pm$ $12.5 \mathrm{mN} \cdot \mathrm{m} \cdot \mathrm{s} \cdot \mathrm{g}^{-1}$ for wild type and $\mathrm{CK}^{-/}$dorsal flexors, respectively. Assuming that the torque time integral normalized per unit weight of tissue linearly relates to chemical energy consumed, it can be estimated that the amount of $\sim \mathrm{P}$ utilised in $\mathrm{CK}^{-/-}$dorsal flexor muscles during the series of 12 contractions will be $69 \%\left(11.5 \mu \mathrm{mol} \cdot \mathrm{g}^{-1}\right.$ d.w. $)$ of that of wild type $\left(16.7 \mu \mathrm{mol} \cdot \mathrm{g}^{-1} \mathrm{~d}\right.$.w). As expected the tissue content of $\mathrm{PCr}$ did not change, underscoring the fact that due to the lack of CK, PCr cannot be used to buffer ATP hydrolysed during mechanical activity of the muscle fibre (6). The concomitant increase in IMP content indicates that (part of) ADP is rephosphorylated by the catalytic action of adenylate kinase (fig. 5.4).

figure 5.4 Schematic representation of the phosphate flux through the adenylate kinase pathway and subsequent reactions in dorsal flexor muscle of $\mathrm{CK}^{-/}$mice during a series of 12 tetanic contractions.

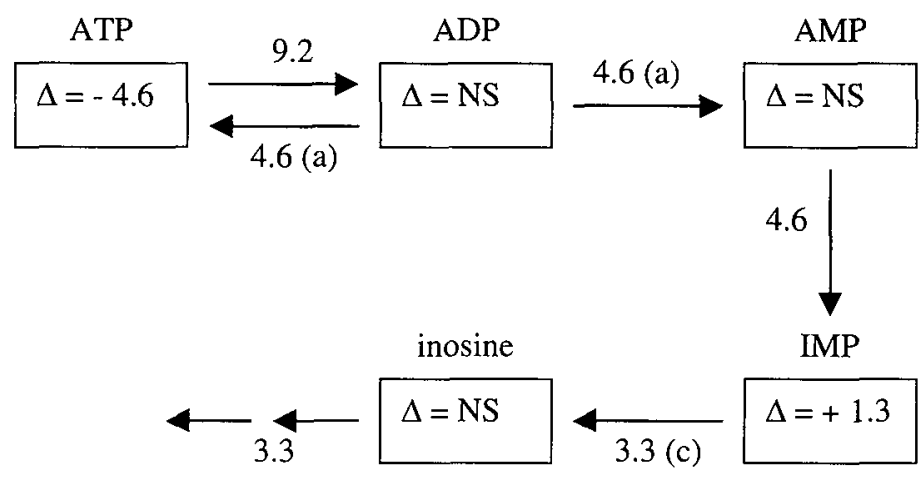

Values are expressed as $\mu \mathrm{mol} \cdot \mathrm{g}^{-1}$ dry weight per contraction series. NS indicates no significant increase (t) or decline (-) in tissue content; a, b, c refer to adenylate kinase, AMP deaminase and 5'nucleotidase, respectively. Note that via the adenylate kinase pathway for each molecule ATP regenerated one molecule AMP is produced and, via AMP deaminase, degraded to IMP and subsequently to purine bases. This process results in a decline of the adenine-nucleotide pool. 
Since for each ATP regenerated via the adenylate kinase pathway one molecule AMP

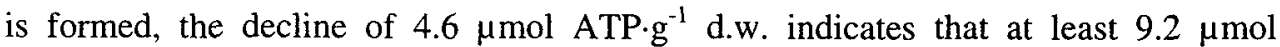
ATP.g ${ }^{-1}$ d.w. is hydrolysed during the series of high-intensity contractions in the $\mathrm{CK}^{-1}$ muscle, neglecting the contribution of the anaerobic glycolysis. This value is in close accordance with the amount of ATP utilised as estimated from the developed torque, i.e., $11.5 \mu \mathrm{mol} \cdot \mathrm{g}^{-1}$ d.w. (see above). It should be emphasised that in $\mathrm{CK}^{-1}$ flexor muscles AMP was not significantly elevated at the end of the contraction series, indicating that AMP once formed is immediately deaminated by action of AMP deaminase. The present finding also show that accumulation of IMP is less than the decline in ATP content, i.e., 1.3 vs. $4.6 \mu \mathrm{mol} \cdot \mathrm{g}^{-1}$ d.w.. This difference strongly suggests that IMP is further degraded to compounds such as inosine, hypoxanthine and xanthine. Chemical analysis of the dorsal flexor muscle tissue at the end of the series of high-intensity contractions did, however, not show an increase in the tissue content of IMP degradation products. This can be explained by the fact that muscular membranes are no barriers for (oxy) purines, allowing the release of these low molecular weight compounds from the contracting muscle into the vascular compartment. The rapid conversion of AMP into IMP and further degradation to (oxy) purines during contractile activity may also explain the substantially lower ATP content in resting muscle of $\mathrm{CK}^{-1-}$ mice compared to wild type. Since the de novo synthesis of ATP via the purine salvage pathway is a relatively slow process (23). This notion is underscored by the present observation that in muscle tissue of $\mathrm{CK}^{-1-}$ mice the sum of adenine nucleotides did not recover during $60 \mathrm{~s}$ rest following the series of tetanic contractions.

The maximal activity of adenylate kinase and AMP deaminase, required for the formation AMP and its subsequent degradation to IMP, was not affected in muscles of $\mathrm{CK}^{-/-}$mice (table 5.3). In vitro studies have shown that the activity of AMP deaminase is, among others, pH dependent (14). Since Steeghs et al. (15) have reported that changes in $\mathrm{pH}$ during mechanical activity of muscles deficient in CK did not differ from wild type, the potential regulatory role of $\mathrm{H}^{+}$can be dismissed under our experimental conditions. Moreover, it is of interest to note that Tullson and co-workers (24) obtained evidence that the affinity of AMP deaminase for AMP is appreciably enhanced in $\mathrm{CK}^{-1-}$ muscles, which may explain the higher rate of IMP formation in the absence of a measurable increase in the tissue content of AMP.

In summary, our results show that the torque output of the in situ dorsal flexor complex of $\mathrm{CK}^{-/}$mice is significantly depressed. This decline can be only partially explained by the decrease in muscle mass. Lower tissue ATP and higher IMP levels may contribute to the decline in torque during a high-intensity tetanic contraction. 
In contrast to wild type muscle ATP content could not be maintained during a series of 12 tetanic contractions in dorsal flexor muscle of $\mathrm{CK}^{-1}$ mice. The increase in tissue IMP indicates that adenylate kinase-mediated regeneration of ATP compensates for the lack of $\mathrm{CK}^{-1-}$ activity. However, this pathway inevitably results in a decline in tissue content of ATP.

\section{6 - Acknowledgement}

The authors thank Prof. dr. B. Wieringa and Mr. F. Oerlemans (Department of Cell Biology and Histology, University of Nijmegen, The Netherlands) for kindly providing us the $\mathrm{CK}$ knockout mice and wild type littermates. 


\section{7 - References}

1. Bessman, S. P., and Carpenter, C. L. (1985) The creatine-creatine phosphate energy shuttle. Annu. Rev. Biochem. 54, 831-862

2. Walliman, T., Wyss, M., Brdiczka, D., and Nicolay, K. (1992) Intracellular compartmentation, structure and function of creatine kinase isoenzymes in tissues with high and fluctuating energy demands: the 'phosphocreatine circuit' for cellular energy homeostasis. Biochem. J. 281, 21-40

3. Wyss, M., Smeitink, J., Wevers, R. A., and Walliman, T. (1992) Mitochondrial creatine kinase: a key enzyme of aerobic energy metabolism. Biochim. Biophys. Acta 1102, 119-166

4. Wyss, M., and Walliman, T. (1994) Creatine metabolism and the consequences of creatine depletion in muscle. Mol. Cell. Biochem. 133/134, 51-66

5. Bessman, S. P., and Geiger, P. J. (1981) Transport of energy in muscle: the phosphorylcreatine shuttle. Science $211,448-452$

6. Steeghs, K., Benders, A., Oerlemans, F., Haan de, A., Heerschap, A., Ruitenbeek, W., Jost, C., Deursen van, J., Perryman, B., Pette, D., Bruckwilder, M., Koudijs, J., Jap, P., Veerkamp, J., and Wieringa, B. (1997) Altered $\mathrm{Ca}^{2+}$ responses in muscles with combined mitochondrial and cytosolic creatine kinase deficiencies. Cell 89, 93-103

7. Dahlstedt, A. J., Katz, A., Wieringa, B., and Westerblad, H. (2000) Is creatine kinase responsible for fatigue? Studies of isolated skeletal muscle deficient in creatine kinase. FASEB J. 14, 982-990

8. Haan de, A., Koudijs, J. C. M., Wevers, R. A., and Wieringa, B. (1995) The effects of MM-creatine kinase deficiency on sustained force production of mouse fast skeletal muscle. Exp. Physiol. 80, 491-494

9. Steeghs, K., Heerschap, A., Haan de, A., Ruitenbeek, W., Oerlemans, F., Deursen van, J., Perryman, B., Pette, D., Bruckwilder, M., Koudijs, J., Jap, P., and Wieringa, B. (1997) Use of gene targeting for comprising energy homeostasis in neuro-muscular tissues. The role of sarcomeric mitochondrial creatine kinase. J. Neurosc. Meth. 71, $29-41$

10. Gorselink, M., Drost, M. R., Louw de, J., Willems, P. J. B., Rosielle, N., Janssen, J. D., and Van der Vusse, G. J. (2000) Accurate assessment of in situ isometric contractile properties of hind limb plantar and dorsal flexor muscle complex of intact mice. Pflïgers Arch. 439, 665-670

11. Edwards, R. H. T., Hill, D. K., and Jones, D. A. (1975) Metabolic changes associated with the slowing of relaxation in fatigued mouse muscle. J. Physiol. 251, 287-301

12. Wijnants, J., and van Belle, H. (1985) Single-run high performance chromatography of nucleotides, nucleosides and major purine bases and its application to different tissue extracts. Anal. Biochem. 144, 258-266

13. Van der Vusse, G. J., Coumans, W. A., Van der Veen, F. H., Drake, A., Flameng, W., and Suy, R. (1984) ATP, creatine phosphate and glycogen content in human myocardial biopsies: markers for the efficacy of cardioprotection. Vasc. Surg. 8, 127134

14. Rundell, K. W., Tullson, P. C., and Terjung, R. L. (1992) Altered kinetics of AMP deaminase by myosin binding. Am. J. Physiol. 263, C294-299

15. Steeghs, K., Oerlemans, F., de Haan, A., Heerschap, A., Verdoodt, L., de Bie, M., Ruitenbeek, W., Benders, A., Jost, C., van Deursen, J., Tullson, P., Terjung, R., Jap, P., Jacob, W., Pette, D., and Wieringa, B. (1998) Cytoarchitectural and metabolic adaptations in muscles with mitochondrial and cytosolic creatine kinase deficiencies. Mol. Cell Biochem. 184, 183-194 
16. Allen, D. G., Lannergren, J., and Westerblad, H. (1995) Muscle cell function during prolonged activity: cellular mechanisms of fatigue. Exp. Physiol. 80, 497-527

17. Westerblad, H., Allen, D. G., Bruton, J. D., Andrade, F. H., and Lannergren, J. (1998) Mechanisms underlying the reduction of isometric force in skeletal muscle. Acta Physiol. Scand. 162, 253-260

18. Kawai, M., Guth, K., Winnikes, K., Haist, C., and Ruegg, J. C. (1987) The effect of inorganic phosphate on the ATP hydrolysis rate and the tension transients in chemically skinned rabbit psoas fibers. Pflugers Arch. 408, 1-9

19. Sahlin, K., Broberg, S., and Ren, J. M. (1989) Formation of inosine monophosphate (IMP) in human skeletal muscle during incremental dynamic exercise. Acta. Physiol. Scand. 136, 193-198

20. Haan de, A. (1990) High-energy phosphates and fatigue during repeated dynamic contractions of rat muscle. Exp. Physiol. 75, 851-854

21. Westra, H. G., Haan de, A., Doorn van, J. E., and Haan de, J. (1990) IMP production and energy metabolism during exercise in rats in relation to age. Biochem. J. 239, 751755

22. Pozzan, T., Rizzuto, R., Volpe, P., and Meldolesi, J. (1994) Molecular and cellular physiology of intracellular calcium stores. Physiol. Rev. 74, 595-636

23. Lowenstein, J. M., and Goodman, M. N. (1978) The purine nucleotide cycle in skeletal muscle. Fed Proc 37, 2308-2312

24. Tullson, P. C., Rush, J. W., Wieringa, B., and Terjung, R. L. (1998) Alterations in AMP deaminase activity and kinetics in skeletal muscle of creatine kinase-deficient mice. Am. J. Physiol. 274, C1411-1416 


\section{Chapter 6}

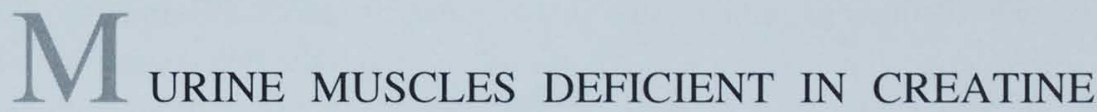
KINASE TOLERATE CONSECUTIVE SERIES OF HIGH-INTENSITY CONTRACTIONS

M. Gorselink, M.R. Drost, Vusse, G.J. van der. (2001) Eur. J. Physiol, under revision.

Muscular performance during two repeated contraction series of creatine kinase deficient and wild type dorsal flexors. 


\section{1 - Summary}

Preliminary findings indicated that during a series of 12 consecutive highintensity contractions $\mathrm{CK}$ deficient $\left(\mathrm{CK}^{-1}\right)$ dorsal flexors could not maintain their ATP content at the pre-contraction level, which was accompanied with a substantial increase in inosine monophosphate (IMP) (chapter 5). Based on these findings, we hypothesised that, first, during a second series of high-intensity contractions maximal force generated during the first contraction would be significantly lower than during the first series because of the putatively depressive effects of low ATP and high IMP levels on muscular contractile behaviour. Second, it was hypothesised that during the subsequent series of high-intensity contractions the tissue ATP pool would be further depleted with a further accumulation of IMP. It was expected that wild type muscles could adequately sustain, both mechanically and biochemically, two repeated series of high-intensity contractions.

The present findings indicate that in $\mathrm{CK}^{--}$muscle maximal torque developed during the first tetanic contraction was comparable between the two contraction series despite a substantial difference in ATP and IMP content at the onset of both series. Also the second hypothesis should be dismissed since ATP content did not further decline $\left(20.6 \pm 1.9,15.5 \pm 2.5,14.4 \pm 3.6\right.$ and $17.3 \pm 3.1 \mu \mathrm{mol} . \mathrm{g}^{-1} \mathrm{dw}$ at rest, at the end of the first contraction series, after $60 \mathrm{~s}$ recovery and at the end of the second series, respectively), while IMP did not further accumulate $(1.2 \pm 0.4,2.4 \pm 1.1,1.8 \pm 1.1$ and $2.5 \pm 1.0 \mu \mathrm{mol} . \mathrm{g}^{-1} \mathrm{dw}$ at rest, at the end of the first contraction series, after $60 \mathrm{~s}$ recovery and at the end of the second series, respectively). The decline in maximal torque during the 12 contractions of the second series was comparable with the decline during the first series. This indicates that elevated IMP and low ATP content do most likely not exert a negative effect on muscle contractile behavior under the present experimental conditions. In contrast, the decline in torque during the $2^{\text {nd }}$ contraction series of wild type muscles $(-20.1 \%)$ was more pronounced than during the $1^{\text {st }}$ series $(-12.3 \%)$, and was accompanied by a substantial increase in the content of IMP. The latter findings indicate that AMP deaminase, in combination with the adenylate kinase pathway, is operational during the $2^{\text {nd }}$, but not during the $1^{\text {st }}$ series of contractions in wild type muscles. 


\section{2 - Introduction}

Phosphocreatine serves as an intracellular high-energy phosphate pool to replenish sarcoplasmic ATP during high-intensity muscular contractions. This process requires creatine kinase (CK), catalysing the reversible phosphotransfer between ATP and phosphocreatine $(1,2)$. Skeletal muscle contains two distinct $\mathrm{CK}$ isoforms, which are localised in the cytosol (MM-CK) and at the interior side of the outer membrane of the mitochondrion $\left(\mathrm{M}_{\mathrm{i}}-\mathrm{CK}\right)(1-4)$. The concerted action of these two isoforms plays an important role in maintaining the cytoplasmic content of high-energy phosphates at physiological levels during episodes of increased muscular energy utilisation (5).

Studies on isolated skeletal muscles deficient in creatine kinase have shown that the inability to regenerate ATP from phosphocreatine results in a significantly lower tetanic force $(6,7)$. Recently we observed that supra-maximal electrical stimulation of the CK deficient dorsal flexor complex in situ resulted in significantly lower maximal torque during a single tetanic contraction (chapter 5). Moreover, during a series of 12 consecutive high-intensity tetanic contractions, $\mathrm{CK}$ deficient $\left(\mathrm{CK}^{-l}\right)$ dorsal flexors could not maintain their ATP content at the pre-contraction value. Furthermore, the decrease in ATP content was accompanied with a substantial increase in the tissue content of inosine monophosphate (IMP). Enhanced IMP content indicates that ATP regeneration through the adenylate kinase pathway $(8,9)$, was not capable of fully compensating the lack of CK. Moreover, after a recovery period of $60 \mathrm{~s}$ following the series of high-intensity contractions, ATP and IMP contents in $\mathrm{CK}^{-1-}$ dorsal flexors did not recuperate towards their pre-contraction values. The altered tissue content of ATP and IMP is supposed to negatively affect muscular contractile performance $(1,5,6,8$, 17).

On the basis of the earlier findings, we hypothesised that, first, in muscle lacking creatine kinase force at the start of a second series of high intensity contractions is substantially lower than at the onset of the first series since ATP and IMP contents do not return to pre-exercise levels during the recovery period. In contrast, force developed in wild type muscle will be identical during the first and second series of high-intensity contractions. Second, it was hypothesised that in $\mathrm{CK}^{-1}$ muscles the tissue content of ATP will further decrease with an additional accumulation of IMP during the second series of high-intensity contractions. These changes will result in a faster decline in developed force during the second series than the first series of contractions. In contrast, the tissue content of ATP in wild type mice was supposed to be maintained during both series of high-intensity contractions since the muscle cells of the latter animals are capable of regenerating ATP from phosphocreatine. 
To test these hypotheses we have subjected the dorsal flexor muscle complex of CK deficient mice to two repeated series of twelve high-intensity isometric contractions, interspaced by a recovery period of $60 \mathrm{~s}$ of rest. Muscular performance was measured with a custom-built mouse dynamometer (10). The muscular content of high-energy phosphates and IMP was determined at the end of the first contraction series, after the first recovery period of $60 \mathrm{~s}$, at the end of the second series of contractions, and at the end after a second period of $60 \mathrm{~s}$ recovery. Resting values were obtained from the contra-lateral muscle group. The same protocol was applied to wild type littermates, serving as controls.

\section{3 - Methods}

\subsubsection{Animals}

Experiments were performed on mice ( $8 \mathrm{wks}$ ) with combined mitochondrial and cytosolic CK deficiency $\left(\mathrm{Mi}_{\mathrm{b}}-\mathrm{CK}^{--}\right.$x $\mathrm{MM}-\mathrm{CK}^{--}$, denoted as $\left.\mathrm{CK}^{-/}\right)$(11). Wild type littermates served as controls. Mice were anaesthetised with 1.5 to $2.0 \%$ halothane (Fluothane ${ }^{\circledR}$, Zeneca, Ridderkerk, The Netherlands), which was supplied in $\mathrm{O}_{2}$ and $\mathrm{N}_{2} \mathrm{O}\left(3: 1,4.01 \cdot \mathrm{min}^{-1}\right)$. All experimental procedures were approved by the Institutional Animal Care and Use Committee of the Maastricht University and complied with the principles of laboratory animal care.

\subsubsection{Surgical procedures and stimulation of the nerve}

Mice were positioned on a thermostatic platform $\left(38.5 \pm 0.1^{\circ} \mathrm{C}\right)$ and after locally depilating the skin, a small incision in the lateral part of the knee was made to expose the peroneal nerve. A bipolar platinum electrode was carefully attached to the nerve and the dorsal muscle complex was stimulated via a pulse generator (HSE 215/IZ, Freiburg, Germany). The position of the electrode was changed if a current of more than $1.0 \mathrm{~mA}$ was needed for supra-maximal stimulation. The electrode was fixated to the skin with cyano-acrylate glue to prevent displacement of the electrode during muscle contraction.

\subsubsection{Experimental measurement set-up}

To assess mechanical performance, dorsal flexor torque around the ankle joint was measured with an isometric dynamometer, the details of which were published earlier (10). Data acquisition was performed at $1000 \mathrm{~Hz}$ with an Apple Macintosh 7100 PowerPC ${ }^{\circledR}$ with an 8 channel, 12 bits Lab-NB analogue-to-digital conversion board (National Instruments ${ }^{\circledR}$, Mopac Expwy, Austin, Tx, USA). Post-processing of the torque data was performed with Matlab 5.3 (The Math Works, Inc., Natick, Massachusetts, USA). 


\subsubsection{Exercise protocol}

Supra-maximal stimulation current, required for full recruitment of muscle fibres, was first determined using 3 to 5 isometric twitch contractions with increasing current. Resting periods between twitches lasted for $60 \mathrm{~s}$. The ankle angle at which torque reached its maximal value, was determined using 9 twitch contractions at ankle angles varying between $15^{\circ}$ dorsal flexion and $25^{\circ}$ plantar flexion. This ankle angle is further denoted as the optimal ankle angle. Maximal tetanic torque was determined during a $150 \mathrm{~ms}$ pulse train at $125 \mathrm{~Hz}$ stimulation frequency. After a 5 min recovery period, dorsal flexors were subjected to two repeated series of 12 isometric tetanic contractions. The muscle was stimulated for $170 \mathrm{~ms}$ every $265 \mathrm{~ms}$ (tour duty cycle: 0.64 ), at optimal ankle angle, $125 \mathrm{~Hz}, 0.5 \mathrm{~ms}$ pulse duration, with a supra-maximal stimulation current. The two contraction series were interspaced by a recovery period of $60 \mathrm{~s}$.

Torque signals of the individual isometric tetanic contractions were digitised and analysed for maximum torque, $1 / 2$-relaxation time, and early and late rise times. Maximum torque was determined from the filtered torque signals (11-point movingaverage), and normalised on the wet mass of the muscle complex. The 1/2-relaxation and the rise times were determined from the unfiltered torque signals. The torque build up during electrical stimulation was quantified as the early and late rise time. The early rise time was calculated as the time required to increase the torque from $10 \%$ to $50 \%$ of its maximal value, whereas the late rise time was calculated as the time required to increase torque from $50 \%$ to $90 \%$ of its maximal value. The $1 / 2$-relaxation time is the time interval during which torque declined from $50 \%$ to $25 \%$ of its maximal value (12).

\subsubsection{Tissue sampling}

Distinct subsets of mice were used for muscular tissue sampling at five timepoints during the two contraction series for the analysis of tissue high-energy phosphates and related compounds, i.e., immediately after the first contraction series (E), $60 \mathrm{~s}$ recovery after $\mathrm{E}(\mathrm{E}+\mathrm{R})$, immediately after the second contraction series $(E+R+E)$, and $60 \mathrm{~s}$ recovery after the second contraction series $(E+R+E+R)$. The muscle complex was freeze-clamped using a pair of metal tongs cooled in liquid nitrogen. In the procedure to take tissue samples immediately after the first or second contraction series ( $E$ and $E+R+E$, respectively), the time interval between the twelfth contraction of the series and the onset of freezing was kept as short as possible $(<2 s)$. Therefore, the contraction protocol for the $\mathrm{E}$ and $\mathrm{E}+\mathrm{R}+\mathrm{E}$ series was performed on a dummy apparatus to prevent damage inflicted upon the sensitive measurement device by the freezing procedure. 
To this end, stimulation conditions, i.e., optimal ankle angle and supra-maximal stimulation current, were determined with the isometric dynamometer and, thereafter, the isometric dynamometer was replaced by the dummy apparatus, keeping the optimal ankle angle, and therefore the muscle length, at optimal value. Muscles were subsequently subjected to either one $(E)$ or two $(E+R+E)$ high-intensity contraction series and rapidly freeze-clamped. Thereafter, the resting contra-lateral dorsal flexor muscle was frozen in liquid nitrogen (C). All tissue samples were stored at $-80{ }^{\circ} \mathrm{C}$ until analysis.

Prior to high-energy phosphate analysis, muscle complexes were freeze-dried overnight at $-30^{\circ} \mathrm{C}$. The tissue content of the high-energy phosphates and related compounds was assessed with a HPLC method $(13,14)$.

\subsubsection{Statistics}

Data are expressed as mean \pm . standard deviation. Differences in high-energy phosphate content at the five time points were tested with a one-way ANOVA with a Tukey post-hoc analysis (C vs. $E, C$ vs. $E+R, E+R$ vs. $E+R+E, E+R$ vs. $E+R+E+R$ ). Differences in mechanical parameters between both series were tested with a Wilcoxon signed ranks test; differences in mass and in mechanical parameters between $\mathrm{CK}^{-/}$and WT with a Mann-Whitney U-test. Differences were considered significant if $\mathrm{p}<0.05$. SPSS 10.0 (SPSS Benelux, Gorinchem, The Netherlands) was used for statistical analyses.

\section{4 - Results}

Body mass of $\mathrm{CK}^{-/-}$mice $(21.3 \pm 1.8 \mathrm{~g})$ was significantly lower than wild type body mass $(24.3 \pm 1.7 \mathrm{~g})$. Dorsal flexor mass of $\mathrm{CK}^{-/}$mice amounted to $53.1 \pm 5.4 \mathrm{mg}$, and was on the average $20 \%$ lower than dorsal flexor mass of age-matched wild type mice $(66.5 \pm 8.3 \mathrm{mg})$.

\subsubsection{Mechanical analysis}

Representative traces of the torque output during the first (A) and second (B) series of 12 isometric tetanic contractions of dorsal flexors of wild type and $\mathrm{CK}^{-/-}$mice are shown in figure 6.1. Both wild type and $\mathrm{CK}^{-1}$ muscles show a decline in maximal torque during the course of the first series of 12 contractions. The torque build up by $\mathrm{CK}^{-1-}$ muscles during the consecutive contractions is slower than by wild type muscles, resulting in the absence of a plateau phase from the $3^{\text {rd }}$ till the $12^{\text {th }}$ contraction. Moreover, relaxation of $\mathrm{CK}$ deficient muscles is appreciably slower than littermate controls. In both wild type and $\mathrm{CK}^{-1-}$ mice, maximal torque of the first contraction did not differ between the first and second series (table 6.1). 
Moreover, the maximal torque during each contraction of both contraction series was found substantially lower in $\mathrm{CK}^{-/}$than in wild type muscles.

figure 6.1 Representative torque output of intact dorsal flexors during the first $(\mathrm{A})$ and second (B) series of 12 isometric tetanic contractions in wild type and $\mathrm{CK}^{-1}$ mice.
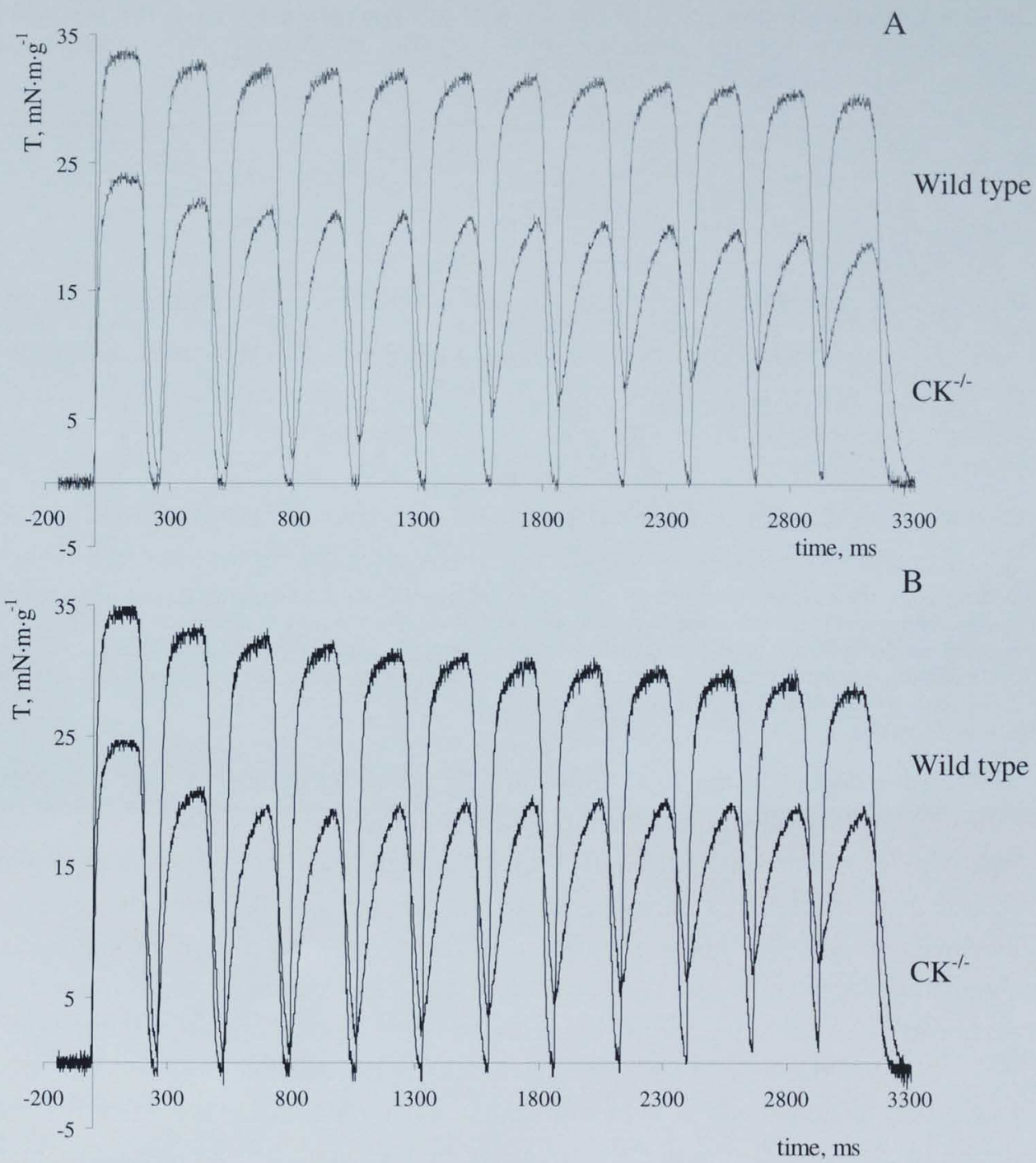

Dorsal flexors were stimulated every $265 \mathrm{~ms}$ for $170 \mathrm{~ms}$ with $125 \mathrm{~Hz}, 0.5 \mathrm{~ms}$, and a supra-maximal stimulation current. The recovery period between the first and second contraction series lasted $60 \mathrm{~s}$. 
Even when normalised on unit muscle mass, the maximal torque in $\mathrm{CK}$ deficient mice was significantly lower (see table 1 for values of the $1^{\text {st }}$ and $12^{\text {th }}$ contraction). For both series, maximal torque during the $12^{\text {th }}$ contraction was lower than during the first contraction both in $\mathrm{CK}^{-/}$and wild type animals (table 6.1).

table 6.1 Contractile parameters of the dorsal flexor complex assessed at the first and last contraction of two repeated contraction series consisting of 12 consecutive isometric tetanic contractions in wild type and $\mathrm{CK}^{-/}$mice.

\begin{tabular}{|c|c|c|c|c|c|c|c|c|}
\hline \multirow{3}{*}{$\begin{array}{l}\text { Group } \\
\text { Contraction \# }\end{array}$} & \multicolumn{4}{|c|}{$1^{\text {st }}$ contraction series } & \multicolumn{4}{|c|}{$2^{\text {nd }}$ contraction series } \\
\hline & \multicolumn{2}{|c|}{ Wild type } & \multicolumn{2}{|c|}{$\mathrm{CK}^{/ \cdot}$} & \multicolumn{2}{|c|}{ wild type } & \multicolumn{2}{|c|}{$\mathrm{CK}^{-1-}$} \\
\hline & $1^{\text {st }}$ & $12^{\text {th }}$ & $1^{\text {st }}$ & $12^{\text {th }}$ & $1^{\mathrm{st}}$ & $12^{\text {th }}$ & $1^{\text {st }}$ & $12^{\text {th }}$ \\
\hline $\begin{array}{l}\text { tetanic torque, } \\
m N \cdot m\end{array}$ & $2.00 \pm 0.25$ & $\begin{array}{c}1.76 \pm 0.29 \\
(\#)\end{array}$ & $\begin{array}{c}1.22 \pm 0.16 \\
(*)\end{array}$ & $\begin{array}{c}0.97 \pm 0.11 \\
(*, \#)\end{array}$ & $2.03 \pm 0.28$ & $\begin{array}{c}1.62 \pm 0.26 \\
(\#)\end{array}$ & $\begin{array}{c}1.23 \pm 0.15 \\
(*)\end{array}$ & $\begin{array}{c}0.94 \pm 0.16 \\
(*, \#)\end{array}$ \\
\hline $\begin{array}{l}\text { torque per unit } \\
\text { mass, } m N \cdot m \cdot g^{-1}\end{array}$ & $30.1 \pm 3.1$ & $\begin{array}{c}26.4 \pm 3.4 \\
(\#)\end{array}$ & $\begin{array}{c}23.0 \pm 3.0 \\
(*)\end{array}$ & $\begin{array}{c}18.3 \pm 2.5 \\
(*, \#)\end{array}$ & $30.5 \pm 4.2$ & $\begin{array}{c}24.4 \pm 3.9 \\
(\#)\end{array}$ & $\begin{array}{c}23.1 \pm 3.0 \\
(*)\end{array}$ & $\begin{array}{c}17.6 \pm 3.0 \\
(*, \#)\end{array}$ \\
\hline $\begin{array}{l}\text { early rise time, } \\
m s\end{array}$ & $11.1 \pm 1.2$ & $10.9 \pm 2.3$ & $10.9 \pm 1.6$ & n.d. & $10.5 \pm 1.2$ & $10.0 \pm 2.3$ & $11.2 \pm 1.1$ & n.d. \\
\hline $\begin{array}{l}\text { late rise time, } \\
m s\end{array}$ & $26.0 \pm 3.8$ & $25.6 \pm 5.5$ & $28.5 \pm 5.3$ & $\begin{array}{c}94.0 \pm 12.7 \\
(*, \#)\end{array}$ & $28.1 \pm 3.9$ & $24.7 \pm 5.3$ & $33.3 \pm 6.1$ & $\begin{array}{c}85.9 \pm 8.8 \\
(*, \#)\end{array}$ \\
\hline $\begin{array}{l}\text { relaxation time, } \\
m s\end{array}$ & $11.1 \pm 2.5$ & $13.9 \pm 3.5$ & $15.0 \pm 2.6$ & $\begin{array}{c}26.2 \pm 2.8 \\
(*, \#)\end{array}$ & $11.8 \pm 3.4$ & $14.4 \pm 1.6$ & $14.9 \pm 2.1$ & $\begin{array}{c}23.8 \pm 2.4 \\
(*, \#)\end{array}$ \\
\hline
\end{tabular}

Dorsal flexors ( $\mathrm{n}=10$ mice in each group) were stimulated with $125 \mathrm{~Hz}, 0.5 \mathrm{~ms}$ and a supra-maximal stimulation current. Duration of the tetanic contraction amounted to $170 \mathrm{~ms}$. The recovery time between the first and second series of contraction lasted $60 \mathrm{~s}$. Statistical differences between wild type (WT) and $\mathrm{CK}^{-1-}$ are indicated by * $(\mathrm{p}<$ 0.05 ), and between the first and $12^{\text {th }}$ contraction of each series by \# $(\mathrm{p}<0.05)$. 'n.d.' means not detectable due to prolonged relaxation time of preceding contraction.

The relative decline in maximal torque in $\mathrm{CK}^{-1-}(20.4 \%)$ during the first contraction series was significantly higher than in wild type muscles (12.3\%) (table 6.2). In wild type muscles, the decline in maximal torque was significantly more pronounced in the second than in the first contraction series. In contrast, in $\mathrm{CK}^{-1-}$ mice the decline in maximal torque was not significantly different between the first and second contraction series (table 2). When wild type and $\mathrm{CK}^{-1-}$ muscles were compared, the decline in maximal torque during the second series of contractions was not different.

The late rise time in $\mathrm{CK}^{-1-}$ mice substantially increased during the course of 12 consecutive contractions, in both contraction series (table 6.1). The early rise time during the $12^{\text {th }}$ contraction in $\mathrm{CK}^{-1-}$ could not be measured, because relaxation of the preceding contraction was prolonged to such extent that the torque did not drop below $10 \%$ of its maximal value. No changes in these temporal parameters occurred in wild type muscles. 
table 6.2 Absolute and relative differences in developed torque between the first and $12^{\text {th }}$ contraction during two repeated series consisting of 12 consecutive isometric tetanic contractions of wild type and $\mathrm{CK}^{-/-}$dorsal flexors. The recovery time interval between the 1 st and 2 nd contraction series lasted $60 \mathrm{~s}$.

\begin{tabular}{|c|c|c|c|c|}
\hline \multirow{2}{*}{$\begin{array}{l}\text { Group } \\
\text { Decline in torque }\end{array}$} & \multicolumn{2}{|c|}{ WT } & \multicolumn{2}{|c|}{$\mathrm{CK}^{\%}$} \\
\hline & $\begin{array}{l}\text { absolute } \\
\left(\mathrm{mN} \cdot \mathrm{m} \cdot \mathrm{g}^{-1}\right)\end{array}$ & $\begin{array}{c}\text { relative } \\
(\%)\end{array}$ & $\begin{array}{c}\text { absolute } \\
\left(\mathrm{mN} \cdot \mathrm{m} \cdot \mathrm{g}^{-1}\right)\end{array}$ & $\begin{array}{c}\text { relative } \\
(\%)\end{array}$ \\
\hline $1^{\text {st }}$ series & $3.7 \pm 2.2$ & $12.3 \pm 7.4$ & $4.7 \pm 1.5$ & $20.4 \pm 6.6^{*}$ \\
\hline $2^{\text {nd }}$ series & $6.1 \pm 2.1 \#$ & $20.1 \pm 6.8 \#$ & $5.5 \pm 2.2$ & $23.8 \pm 9.5$ \\
\hline
\end{tabular}

Data are expressed as mean \pm sd. Statistical differences between wild type (WT) and $\mathrm{CK}^{\%} \quad(\mathrm{n}=10$ mice in each group) are indicated by * $(\mathrm{p}<0.05)$, and differences between the first and second exercise series are indicated by $\#(\mathrm{p}<0.05)$.

\subsubsection{Muscle high-energy phosphate content}

Table 6.3 summarises the dorsal flexor high-energy phosphate content in wild type and $\mathrm{CK}^{-1-}$ mice at distinct time points during the experimental protocol.

ATP: ATP values in wild type muscles remained constant during the course of the contraction series. The resting muscular ATP content of $\mathrm{CK}^{-1-}$ mice was significantly lower than in wild type. The $\mathrm{CK}^{-1-}$ muscles showed a contraction-induced decrease in ATP content during the first series $\left(\Delta \mathrm{ATP}=-5.1 \mu \mathrm{mol} \cdot \mathrm{g}^{-1} \mathrm{~d}\right.$.w. $)$. Moreover, the decreased ATP content did not recuperate during the recovery period $(E+R)$. Surprisingly, ATP levels in $\mathrm{CK}^{-/}$dorsal flexors did not decline further during the second series of contractions $(E+R+E)$.

ADP and AMP: Resting ADP and AMP content was significantly lower in wild type than in $\mathrm{CK}^{-1}$ muscles. The ADP as well as the AMP content in wild type muscles remained constant throughout the course of the contraction protocol. In $\mathrm{CK}^{-/}$muscles, the content of ADP and AMP remained unchanged during the first contraction series, while the content of both ADP and AMP showed a decline compared to precontraction values during the recovery period $(E+R)$.

IMP: Basal IMP content was significantly higher in $\mathrm{CK}^{-/}$than in wild type mice. Compared to the pre-contraction content $(C)$, wild type mice showed no change in IMP content during $\mathrm{E}$ and $\mathrm{E}+\mathrm{R}$. In contrast, $\mathrm{CK}^{-/}$muscles showed a significant increase in IMP content during the first contraction series $(E)$, whereas the IMP content remained elevated after the $60 \mathrm{~s}$ recovery period $(\mathrm{E}+\mathrm{R})$. Interestingly, the second contraction series resulted in a significant increase in IMP content $(2.6 \pm 2.1$ $\mu$ mol. $\mathrm{g}^{-1}$ d.w.) in wild type mice, whereas IMP content in $\mathrm{CK}^{-/}$muscles remained stable at $2.5 \pm 1.0 \mu \mathrm{mol} \cdot \mathrm{g}^{-1} \mathrm{~d}$.w. Muscular IMP content in both wild type and $\mathrm{CK}^{-1-}$ muscles remained significantly elevated compared to pre-contraction values, i.e., $2.0 \pm$ 1.4 and $2.4 \pm 0.4 \mu \mathrm{mol} \cdot \mathrm{g}^{-1} \mathrm{~d}$.w., respectively, during the second recovery period. 


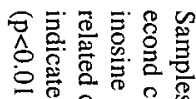
을

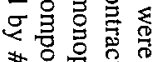

* $\hat{0}$ 的要 还尔

言然西要

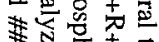
证古声

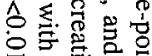

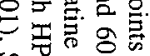

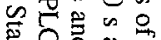
를 递兽 四

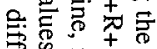

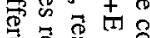

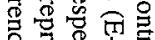
象 可

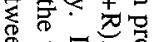
\% 응 ) 을 总器 미의.

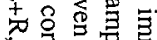

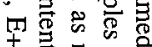

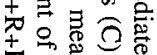

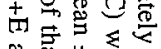

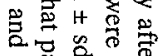
of है을 역 罗8 8 卉密 항

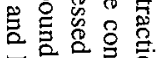
界

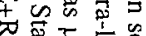
南爱

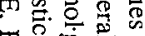

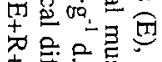
南需的器

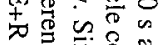

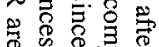

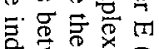

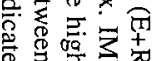

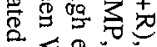

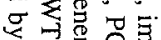
* 或 这嗐言

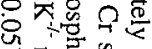

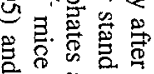

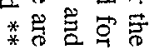

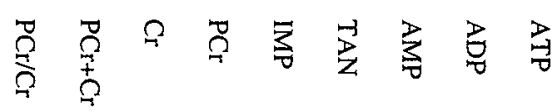

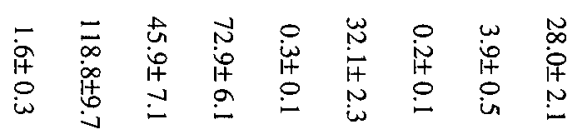

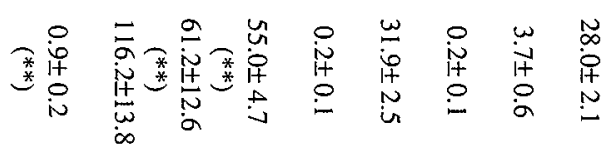

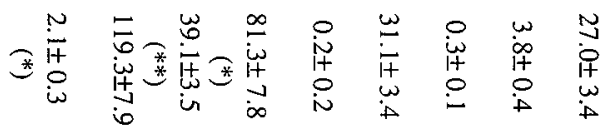

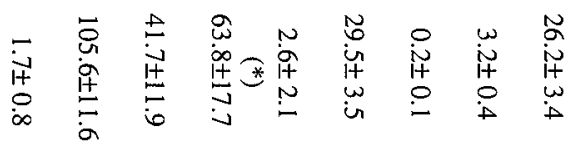

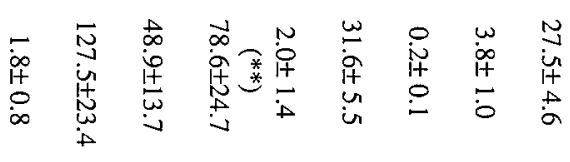

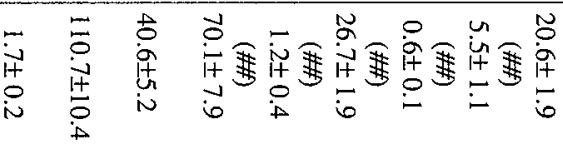

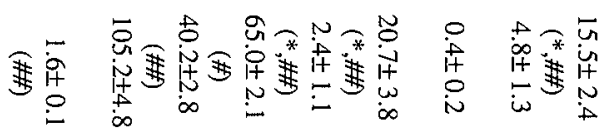

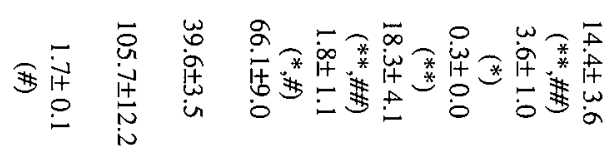

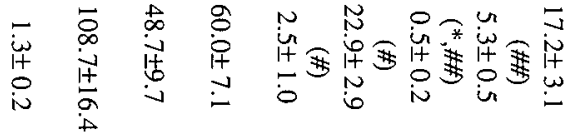

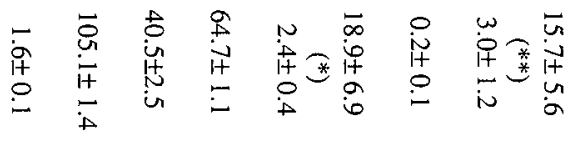


PCr and Cr: $\mathrm{CK}^{-/}$muscle showed normal resting phosphocreatine (PCr) and creatine (Cr) contents, which resulted in $\mathrm{PCr} / \mathrm{Cr}$ ratios comparable with wild type. Immediately after the first contraction series, $\mathrm{PCr}$ content in wild type muscle decreased significantly $\left(\Delta \mathrm{PCr}=-17.9 \mu \mathrm{mol} \cdot \mathrm{g}^{-1}\right.$ d.w.), whereas the $\mathrm{Cr}$ content showed a reciprocal increase $\left(\Delta \mathrm{Cr}=+15.3 \mu \mathrm{mol} \cdot \mathrm{g}^{-1} \mathrm{~d}\right.$.w. $)$. The $\mathrm{PCr} / \mathrm{Cr}$ ratio of wild type mice decreased significantly from $1.6 \pm 0.3$ in resting muscle towards $0.9 \pm 0.2$ at the end of the contraction series. In contrast, the $\mathrm{PCr}$ and $\mathrm{Cr}$ content during $\mathrm{E}$ in $\mathrm{CK}^{-/}$muscles did not change. During the $60 \mathrm{~s}$ recovery period $(\mathrm{E}+\mathrm{R}), \mathrm{PCr}$ content increased and $\mathrm{Cr}$ content in wild type muscle complex decreased to such extent that resting values were overshoot. These changes resulted in a significantly increased $\mathrm{PCr} / \mathrm{Cr}$ ratio when compared to the pre-contraction values (C). Similar to the first contraction series $(E)$, no significant changes occurred in $\mathrm{PCr}$ and $\mathrm{Cr}$ content in $\mathrm{CK}^{-/}$mice during the second series of tetanic contractions $(\mathrm{E}+\mathrm{R}+\mathrm{E})$. The mean $\mathrm{PCr}$ content of wild type dorsal flexors substantially decreased, but due to the large inter-individual variation this decline did not reach the level of significance. During $\mathrm{E}+\mathrm{R}+\mathrm{E}+\mathrm{R}$, the $\mathrm{PCr}$ and $\mathrm{Cr}$ contents in wild type muscles returned to basal values.

\section{5 - Discussion}

Muscles lacking both mitochondrial and cytoplasmic creatine kinase generate a significantly lower force during a tetanic contraction than wild type muscles $(7,11)$. Moreover, the basal ATP levels are significantly lower and tissue IMP content is higher in $\mathrm{CK}^{--}$than in wild type muscles. The present findings clearly indicate that in muscles lacking both creatine kinase isoforms maximal torque developed during the first tetanic contraction of a second series of high-intensity contractions is comparable with that of the first series from which it is divided by a recovery period of $60 \mathrm{~s}$. The fact that the tissue content of ATP is appreciably lower and that of IMP significantly higher at the onset of the second contraction series than at the start of the first series makes a prominent role of these two substances in the decline in torque in $\mathrm{CK}^{-1-}$ muscles, when compared to wild type, less likely.

Contrary to our expectations, the tissue content of ATP did not further decline and that of IMP not further increase during the second series of 12 high-intensity contractions. This phenomenon was accompanied by a comparable degree of decline of maximal torque in $\mathrm{CK}^{-/}$during the 12 consecutive contractions muscles of the second series when compared to the first series. In contrast, in muscles of wild type mice IMP accumulated and maximal torque showed a more pronounced decline during the second contraction series, when compared to the first contraction series. 
As indicated above, resting muscular ATP content was significantly lower in $\mathrm{CK}^{-/-}$than in wild type mice, while the opposite was true for the IMP content. The content of phosphocreatine and creatine was comparable between $\mathrm{CK}^{-/-}$and wild type muscles. These observations in resting muscles corroborate findings earlier published by Steeghs and colleagues (11), although differences in absolute values between the two studies exist. These differences are most likely caused by differences in analytical techniques employed to monitor the tissue content of energy phosphates and related compounds. Steeghs et al. (11) applied enzymatic techniques while an HPLC method was used in the present study. Despite the presence of a physiological amount of $\mathrm{PCr}$ in the CK deficient dorsal flexor muscles, this high-energy phosphate is obviously not available to regenerate ATP during high-intensity contractions due to the lack of creatine kinase activity. The latter finding is in accordance with earlier observations (7).

The present findings show that during the first contraction series, lack of $\mathrm{CK}$ activity results in a significant decline in the muscular ATP content with a concomitant increase in tissue IMP. Since these changes were not restored during the 60 s recovery phase, the second contraction series may start under unfavourable conditions as others have suggested that enhanced tissue IMP content either or not in combination with a low ATP content depresses muscular contractility $(8,17)$. The observation that the maximal torque of the first contraction of the 1 st and 2 nd contraction series were comparable indicates that, at least under the present conditions, the higher IMP content and lower ATP content do most likely not negatively affect muscular contractile performance.

Furthermore, the present data suggest that during the first contraction series of $\mathrm{CK}^{-1-}$ muscles a substantial part of ATP is most likely regenerated by the catalytic action of adenylate kinase. In this pathway, two molecules of ADP are converted into ATP and AMP (fig. 6.2). The net decline in tissue ATP content and concomitant accumulation of IMP indicate that the contribution of alternative sources of ATP, for instance the mitochondrial oxidative phosphorylation or substrate-linked phosphorylation in the glycolytic pathway (15), is insufficient to maintain the cellular ATP pool during high-intensity contractions. Therefore, AMP cannot or only partially be reconverted into ADP by a second set of adenylate kinase enzymes, which are closely associated with the glycolytic pathway, as indicated on the left side of figure 6.2. According to this metabolic model, which was first proposed by Bessman and Carpenter (1) and later extended by Zeleznikar and co-workers (17), ADP formation from AMP requires a continuous supply of ATP (see below for more detailed discussion). Under the present conditions AMP is, however, deaminated by the action of AMP deaminase, yielding IMP (16). 
figure 6.2 Proposed mechanism for adenylate kinase-catalysed high-energy phosphate transfer (adapted from reference 17).

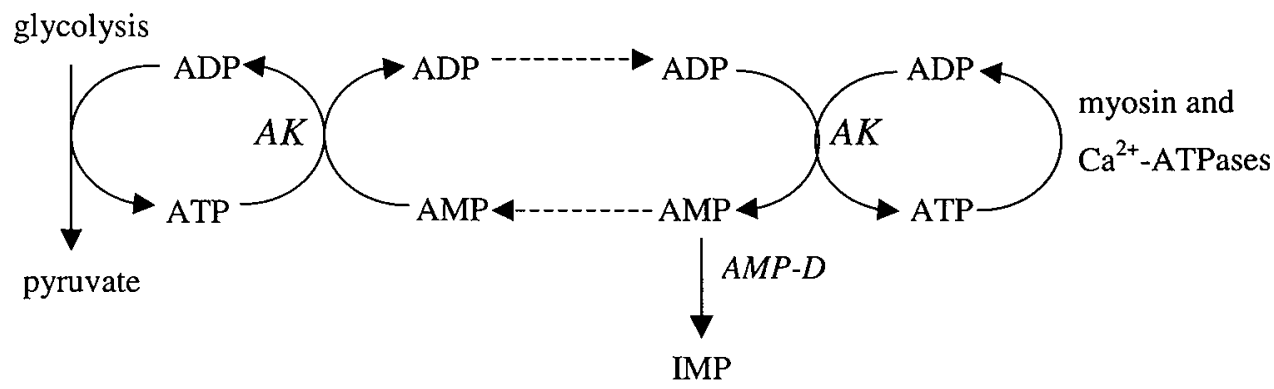

AK and AMP-D refer to adenylate kinase and AMP deaminase, respectively. Solid arrows indicate metabolic conversion and broken arrows indicate diffusion of the chemical substance

The inherent disadvantage of this kind of ATP regeneration in $\mathrm{CK}^{-/-}$muscles is a drain of adenine nucleotides from the high-energy phosphate pool. This drain resulted in an approximately $25 \%$ decline in ATP content during the first series of high-intensity contractions.

Since during the second series of high-intensity contractions in $\mathrm{CK}^{-/-}$muscle no net decline of ATP content and further accumulation of IMP occurred, despite the fact that mechanical performance was comparable between the two contraction series, an alternative source of ATP became obviously available to fully meet the energy request of the contracting muscle cell. This source is most likely substrate-linked phosphorylation in the glycolytic pathway. A substantial contribution of oxidative phosphorylation is less likely since the muscle performed high-intensity contractions during a relatively short period of time (about $3 \mathrm{~s}$ ) with a tour duty cycle of 0.64 . According to the model of Zeleznikar and co-workers (17), ATP produced via the glycolytic pathway reacts in the cytoplasm with AMP yielding two molecules of ADP. This reaction step is catalysed by adenylate kinase associated with the glycolytic complex (fig. 6.2). One ADP molecule diffuses to the contractile machinery, i.e., the site of ATP consumption, and reacts with a second ADP molecule, which is produced during muscular contraction. This reaction, catalysed by a second set of adenylate kinase enzymes, yields ATP and AMP. ATP is subsequently hydrolysed by, for instance, myosin ATPases or sarcoplasmic reticular $\mathrm{Ca}^{2+}$ ATPases to ADP. AMP diffuses back through the cytoplasm to react with ATP, newly formed in the glycolytic pathway (fig. 6.2). In this cyclic process, AMP obviously evades the AMP deaminasecatalysed conversion into IMP. It remains, however, unclear why the alternative source of ATP (most likely glycolysis) is insufficiently active during the first series of contractions in the CK deficient muscle. 
It is tempting to speculate that factors mobilised during the first contraction series are required to pre-condition the muscular tissue to adequately generate energy for subsequent series of high-intensity contractions, e.g. phosphorylation of regulatory proteins such as glycogen phosphorylase. However, the precise nature of these factors and mechanisms involved require further detailed investigations.

While muscle lacking creatine kinase activity appears to be able to tolerate a repeated series of high-intensity contractions, the same challenge exerted on normal muscle revealed both biochemically and mechanically a less adequate response. The more pronounced rate of decline of maximal torque developed during the second series indicates a higher degree of fatigability than during the first series. Moreover, the significant increase in tissue IMP content in combination with a decline in the content of $\mathrm{PCr}$ during the second series shows that, in contrast to the first series, the creatine kinase-mediated regeneration of ATP is not sufficient to fully meet the energy demands of the contracting muscle. It should be stressed that the increase in IMP content is accompanied by a quantitatively comparable, although statistically not significant, decline of the sum of the tissue content of ATP, ADP, and AMP. This indicates that, on the one hand, further degradation of IMP to (oxy)purines does most likely not occur and, on the other, the contribution of the adenylate kinase plus AMP deaminase pathway contribute only little to the overall ATP regeneration. The most important contributor is most likely still creatine kinase-mediated rephosphorylation of ADP.

It is of interest to note that Dahlstedt and colleagues (6) recently observed that with repeated tetanic stimulation fast-twitch muscle fibres fatigue more slowly in CK deficient than in wild type mice. In this respect our findings in intact muscles in situ during the second series of high-intensity contractions corroborate with the observations of Dahlstedt and co-workers in isolated myocytes and isolated whole muscles. The cause of fatigue is most likely multifactorial $(6,16)$, but Dahlstedt and colleagues have pointed out that the accumulation of inorganic phosphate may play a particular role in the process of muscular fatigue (6). They explained the increased fatigue resistance of $\mathrm{CK}^{-1}$ deficient muscle during prolonged electrical stimulation by a less pronounced production of inorganic phosphate from phosphocreatine due to the absence of creatine kinase activity (4). If true, it remains, however, to be elucidated why in wild type mice this mechanism is not operational during the first series of contractions in our experimental set up since the decline in the content of $\mathrm{PCr}$ was comparable between both contraction series. 
Theoretically, changes in muscular fibre type in CK deficient muscle may also contribute to the differences observed between $\mathrm{CK}^{-/}$and wild type animals. This possibility, however, should be dismissed since Steeghs and co-workers have shown that in muscles of mice, lacking both mitochondrial and cytosolic creatine kinase, fibre type shifts did not occur (11).

In summary, we hypothesised that muscle lacking both creatine kinase isoforms will rapidly lose their ability to generate force when subjected to repeated series of high-intensity contractions. Moreover, it was hypothesised that in $\mathrm{CK}^{-/}$muscles a second series of contractions would further deplete the muscular ATP pool with a concomitant rise in tissue content of IMP. The present findings, however, indicate that in $\mathrm{CK}^{-/}$mice the initial torque output of the in situ dorsal flexor complex in the second contraction series was comparable with that of the first contraction series. It was also observed that the tissue contents of ATP and IMP remained stable during the second series of high-intensity contractions. In contrast, wild type muscles showed an increase in muscular fatigue during the second contraction series in combination with an elevated tissue IMP content.

\section{6 - Acknowledgement}

The authors thank Prof. dr. B. Wieringa (Department of Cell Biology and Histology, University of Nijmegen, The Netherlands) for kindly providing us the CK knockout mice and wild type littermates. 


\section{7 - References}

1. Bessman, S. P., and Carpenter, C. L. (1985) The creatine-creatine phosphate energy shuttle. Annu. Rev. Biochem. 54, 831-862

2. Walliman, T., Wyss, M., Brdiczka, D., and Nicolay, K. (1992) Intracellular compartmentation, structure and function of creatine kinase isoenzymes in tissues with high and fluctuating energy demands: the 'phosphocreatine circuit' for cellular energy homeostasis. Biochem. J. 281, 21-40

3. Wyss, M., and Walliman, T. (1994) Creatine metabolism and the consequences of creatine depletion in muscle. Mol. Cell. Biochem. 133/134, 51-66

4. Wyss, M., Smeitink, J., Wevers, R. A., and Walliman, T. (1992) Mitochondrial creatine kinase: a key enzyme of aerobic energy metabolism. Biochim. Biophys. Acta 1102, 119-166

5. Bessman, S. P., and Geiger, P. J. (1981) Transport of energy in muscle: the phosphorylcreatine shuttle. Science $211,448-452$

6. Dahlstedt, A. J., Katz, A., Wieringa, B., and Westerblad, H. (2000) Is creatine kinase responsible for fatigue? Studies of isolated skeletal muscle deficient in creatine kinase. FASEB J. 14, 982-990

7. Steeghs, K., Benders, A., Oerlemans, F., Haan de, A., Heerschap, A., Ruitenbeek, W., Jost, C., Deursen van, J., Perryman, B., Pette, D., Bruckwilder, M., Koudijs, J., Jap, P., Veerkamp, J., and Wieringa, B. (1997) Altered $\mathrm{Ca}^{2+}$ responses in muscles with combined mitochondrial and cytosolic creatine kinase deficiencies. Cell 89, 93-103

8. Tullson, P. C., and Terjung, R. L. (1991) Adenine nucleotide metabolism in contracting skeletal muscle. Ex. Sport Sci. Rev. 19, 507-537

9. Dzeja, P. D., Zeleznikar, R. J., and Goldberg, N. D. (1996) Suppression of creatine kinase-catalyzed phosphotransfer results in increased phosphoryl transfer by adenylate kinase in intact skeletal muscle. J. Biol. Chem. 271, 12847-12851

10. Gorselink, M., Drost, M. R., Louw de, J., Willems, P. J. B., Rosielle, N., Janssen, J. D., and Van der Vusse, G. J. (2000) Accurate assessment of in situ isometric contractile properties of hind limb plantar and dorsal flexor muscle complex of intact mice. Pflügers Arch. 439, 665-670

11. Steeghs, K., Heerschap, A., Haan de, A., Ruitenbeek, W., Oerlemans, F., Deursen van, J., Perryman, B., Pette, D., Bruckwilder, M., Koudijs, J., Jap, P., and Wieringa, B. (1997) Use of gene targeting for comprising energy homeostasis in neuro-muscular tissues. The role of sarcomeric mitochondrial creatine kinase. J. Neurosc. Meth. 71, $29-41$

12. Edwards, R. H. T., Hill, D. K., and Jones, D. A. (1975) Metabolic changes associated with the slowing of relaxation in fatigued mouse muscle. J. Physiol. 251, 287-301

13. Van der Vusse, G. J., Coumans, W. A., Van der Veen, F. H., Drake, A., Flameng, W., and Suy, R. (1984) ATP, creatine phosphate and glycogen content in human myocardial biopsies: markers for the efficacy of cardioprotection. Vasc. Surg. 8, 127134

14. Wijnants, J., and van Belle, H. (1985) Single-run high performance chromatography of nucleotides, nucleosides and major purine bases and its application to different tissue extracts. Anal. Biochem. 144, 258-266

15. Savabi, F. (1994) Interaction of creatine kinase and adenylate kinase systems in muscle cells. Mol. Cell. Biochem. 133/134, 145-152 
16. Lowenstein, J. M. (1972) Ammonia production in muscle and other tissues: the purine nucleotide cycle. Physiol. Rev. 52, 382-414

17. Zeleznikar, R. J., Dzeja, P. P., and Goldberg, N. D. (1995) Adenylate kinase-catalyzed phosphoryl transfer couples ATP utilization with its generation by glycolysis in intact muscle. J Biol Chem 270, 7311-7319 
Chapter 6 


\section{Chapter 7}

IFFERENTIAL RESPONSE TO CREATINE KINASE ABLATION BETWEEN DORSAL AND PLANTAR FLEXORS IN MICE

Effect of creatine kinase ablation on contractile performance of the plantar and dorsal flexor muscle complex in situ. 


\section{1 - Summary}

In the present study, we have investigated whether the dorsal and plantar flexor complexes respond differently towards a combined ablation of mitochondrial and cytosolic creatine kinase $\left(\mathrm{CK}^{-/}\right)$. The mechanical properties of these muscle complexes were assessed with a newly developed mouse isometric dynamometer (1) keeping the intact muscle in its physiological environment. Moreover, mechanical functioning was related to myosin heavy chain (MyHC) composition, to basal high-energy phosphate levels, and maximal activity of a set of enzymes involved in oxidative metabolism.

Maximal torque per unit muscle mass of plantar and dorsal flexors of wild type mice during a single tetanic contraction amounted to $62.7 \pm 9.3$ and $31.7 \pm 3.5$ $\mathrm{mN} \cdot \mathrm{m} \cdot \mathrm{g}^{-1}$. In $\mathrm{CK}^{-1-}$ mice, the decline in torque per unit mass was comparable in plantar and dorsal flexors, and decreased to $53.6 \pm 10.0$ and $23.0 \pm 2.9 \mathrm{mN} \cdot \mathrm{m} \cdot \mathrm{g}^{-1}$, respectively. The decrease in resting ATP content in $\mathrm{CK}^{-1-}$ mice did not significantly differ between the plantar $(-18 \%)$ and dorsal flexors $(-26 \%)$. Furthermore, resting IMP levels in both $\mathrm{CK}^{-/-}$muscle complexes exhibited a fourfold increase. During a series of 12 consecutive contractions, the torque of intact plantar flexors exhibited a substantial decline already during the second contraction, which contrasted with the gradual decline in torque observed in the dorsal flexors of $\mathrm{CK}^{-/}$mice. This differential response to $\mathrm{CK}$ ablation was found to be associated with a shift of the type IIB MyHC isoform towards the slower type IIA/X isoform in dorsal flexors, whereas no changes in $\mathrm{MyHC}$ were observed in plantar flexors. Moreover, gastrocnemius plus plantaris muscles and dorsal flexors of $\mathrm{CK}^{-/}$mice showed an increase in the mitochondrial enzyme citrate synthase, in hydroxyacyl-CoA dehydrogenase, and in the cytoplasmic fatty acid binding protein. It is, however, unlikely that the latter adaptations explain the differential response between $\mathrm{CK}^{-/}$plantar and dorsal flexors during a repeated contraction series. 


\section{2 - Introduction}

Creatine kinase (CK) forms a small family of muscle specific cytosolic (MM$\mathrm{CK})$ and mitochondrial $\left(\mathrm{M}_{\mathrm{i}}-\mathrm{CK}\right)$ isoenzymes $(2,3)$, which play a paramount role in maintaining sarcoplasmic adenine nucleotide levels at rest, and during exercise (4). Recently, Steeghs and co-workers reported that force development by the medial gastrocnemius, isolated from $\mathrm{M}_{\mathrm{i}} \mathrm{CK}$ and $\mathrm{MM}-\mathrm{CK}$ deficient mice $\left(\mathrm{CK}^{-l}\right)$, was significantly depressed $(5,6)$. Moreover, during a series of isometric contractions, the isolated medial gastrocnemius muscle exhibited a fast and substantial decline in force already during the second contraction, i.e., a phenomenon denoted as 'lack of burst activity' $(6,7)$. In contrast, we recently observed that in $\mathrm{CK}^{-/-}$mice the intact dorsal flexor complex, which consists of tibialis anterior and extensor digitorum longus (EDL) muscles, showed a slow and progressive decline in performance during a series of isometric contractions (8). Theoretically, these striking differences may be caused by either differences in experimental set-up, i.e., isolated muscle vs. intact muscle complex, or by differences in muscle properties studied, i.e., the medial gastrocnemius muscle, being part of the plantar flexor complex vs. the intact dorsal flexor complex. In the present study, we have investigated whether the dorsal and plantar flexor complexes respond differently towards a combined ablation of mitochondrial and cytosolic creatine kinase. The mechanical properties of these muscle complexes were assessed with a newly developed mouse isometric dynamometer (1) keeping the intact muscle in its physiological environment. Moreover, we have explored whether differences in mechanical behaviour, if any, are related to adaptational changes in muscles of $\mathrm{CK}^{-/-}$mice. These potential adaptations include changes in the basal tissue content of high-energy phosphates prior to contraction, in the activity of a set enzymes involved in energy conversion, and in the myosin heavy chain (MyHC) composition of the muscle complexes under investigation $(9,10)$.

\section{3 - Methods}

\subsubsection{Animals}

Mice lacking both mitochondrial and cytosolic $\mathrm{CK}\left(\mathrm{M}_{\mathrm{i}}-\mathrm{CK}^{-/-} \times \mathrm{MM}-\mathrm{CK}^{-1-}\right.$, denoted as $\mathrm{CK}^{-/}$) were used in the present study (6). All experiments were performed on 8 weeks old male wild-type mice (C57/B16 x 129/sv) and age-matched $\mathrm{CK}^{-/}$mice. Mice were anaesthetised with 1.5 - 2.0\% Halothane (Fluothane ${ }^{\circledR}$, Zeneca, Ridderkerk, The Netherlands) which was mixed with $\mathrm{O}_{2}$ and $\mathrm{N}_{2} \mathrm{O}\left(3: 1,4.0 \mathrm{l} \cdot \mathrm{min}^{-1}\right)$, and delivered to the mouse via a facemask connected to a flow meter system (Medec, Montvalle, N.J., USA). 
All experimental procedures were approved by the Institutional Animal Care and Use Committee of the Maastricht University and complied with the principles of proper laboratory animal care.

\subsubsection{Surgical procedure}

Plantar or dorsal muscle complexes were stimulated via the tibial or peroneal nerve, respectively. After depilating the skin, a small incision in the hollow of the knee or in the lateral part of the knee was made to obtain access to the tibial or peroneal nerve, respectively. A bipolar platinum hook electrode, interpole distance $0.8 \mathrm{~mm}$, was carefully attached to the nerve and the muscle complex was stimulated using a pulse generator (HSE 215/IZ, Freiburg, Germany). To prevent electrode displacement during muscle contraction the electrode was fixated to the skin with cyano-acrylate glue.

\subsubsection{Experimental protocol}

To assess the mechanical properties of the muscle complexes, the anaesthetised mouse was fixated at the hip and foot to the measurement device. Plantar and dorsal flexor torque were measured in random order with the isometric mouse dynamometer, the details of which were published earlier (1). First, supra-maximal stimulation current, necessary to obtain recruitment of all muscle fibres, was determined by eliciting 5 isometric twitch contractions with increasing current. Then optimal angle, at which the muscle complex exerted the maximal torque, was determined using 13 twitch contractions at ankle angles between $30^{\circ}$ dorsal flexion and $30^{\circ}$ plantar flexion. Resting periods between twitches were kept constant at $60 \mathrm{~s}$. Maximal tetanic torque was obtained with a $200 \mathrm{~ms}$ pulse train at 125 or $100 \mathrm{~Hz}$ stimulation frequency for the dorsal flexors and the plantar flexors, respectively. Following a 10 min resting period, the plantar or dorsal flexors were subjected to a series of 12 isometric tetanic contractions for $170 \mathrm{~ms}$ every $265 \mathrm{~ms}$ (tour duty cycle: 0.64 ), at optimal ankle angle, $100 \mathrm{~Hz}$ and $125 \mathrm{~Hz}$ for the plantar and dorsal flexors, respectively. The pulse duration amounted to $0.5 \mathrm{~ms}$. Torque signals were digitised and analysed for maximal tetanic torque, rise time, and relaxation time. Maximal torque was determined from filtered torque signals during electrical stimulation. The rise time and the relaxation time were determined from the unfiltered torque signals. The rise time is defined as the time required to increase the muscle torque from 10 to $50 \%$ of its maximum value. The relaxation time is the time to decrease the torque from 50 to $25 \%$ of the maximum value after cessation of electrical stimulation (11). Furthermore, the maximal torque value of the 12 contractions was determined and normalised to the maximal torque of the initial contraction. 
Temporal properties of torque development were characterised in terms of rise time and relaxation time of the initial and $12^{\text {th }}$ contraction of the contraction series.

\subsubsection{Tissue sampling and biochemical analysis}

At the end of the experiment, both the dorsal and plantar muscle complexes were dissected from the resting contra-lateral hind limb and rapidly freeze-clamped using a pair of liquid nitrogen cooled metal tongs. Prior to high-energy phosphate analysis, the muscle complexes were freeze-dried overnight at $-30^{\circ} \mathrm{C}$. High-energy phosphates and related compounds were assessed with a high performance liquid chromatography (HPLC) method (12). Total adenine nucleotide content (TAN) was computed as the sum of ATP, ADP, and AMP.

For the analysis of myosin heavy chain (MyHC) isoform composition, the midbelly regions of exercised muscle complexes were snap frozen in melting isopentane. Transverse cryosections of $10 \mu \mathrm{m}$ of the gastrocnemius plus plantaris muscles, soleus muscle, and tibialis anterior plus extensor digitorum longus (EDL) muscles were stored in sample buffer (glycerol, SDS (10\%), Tris $0.5 \mathrm{M} \mathrm{pH} \mathrm{6.8,} \mathrm{Bromphenol} \mathrm{blue,}$ dithiotreitol) at $-80{ }^{\circ} \mathrm{C}$ until analysis of $\mathrm{MyHC}$ isoform composition. MyHC composition of the muscles was determined by SDS-polyacrylamide gel electrophoresis. Briefly, total acrylamide concentration was $4 \%$ in the stacking gel, and $7 \%$ in the running gel. The gel matrix included $30 \%$ of glycerol. Electrophoresis was performed at $120 \mathrm{~V}$ for $27 \mathrm{~h}$ with a tris (hydroxymethyl)aminomethane-glycine electrode buffer ( $\mathrm{pH} 8.3$ ) at $15^{\circ} \mathrm{C}(13)$. The gels were silver stained (Biorad Silver Plus Kit, Veenendaal, The Netherlands) and scanned in a laser densitometer (Fluor-s, Multilmager, Biorad, Veenendaal, The Netherlands) to determine the relative proportions of distinct MyHC isoforms, i.e., type I, IIA/X, and IIB (13).

One set of contra-lateral plantar and dorsal flexor muscles from wild type and $\mathrm{CK}^{-1-}$ mice were used to determine the maximal activity of total creatine kinase (CK), total AMP deaminase (AMP-d), citrate synthase (CS), and 3-hydroxyacyl-CoA dehydrogenase (HAD). Briefly, muscle complexes were homogenised in a sucrose (250 mM), EDTA (2 mM) and Tris-HCl (10 mM, pH 7.4) (SET) buffer. Measurement of maximal CK activity (EC 2.7.3.2.) was based on a coupled enzyme assay of hexokinase (EC 2.7.1.1) and glucose-6-phosphate dehydrogenase (EC 1.1.1.49) producing NADPH. AMP deaminase (EC 3.5.4.6) activity was assessed according to Rundell and co-workers (14). CS activity measurements were based on adding acetylCoA, oxaloacetate and 5,5'-dithiobis(2-nitrobenzoic acid) (DTNB) to the sample. CS converts acetyl-CoA and oxaloacetate to citrate and $\mathrm{CoA}-\mathrm{SH}$. The latter binds to DTNB to form CoA-DTNB. The determination of HAD activity was based on the conversion of acetoacyl-CoA and NADH to 3-hydroxybutyryl-CoA and NAD. 
The enzyme activities were measured with a centrifugal analyser (Cobas Tara, LaRoche, Switzerland) and expressed as $\mathrm{U} \cdot \mathrm{g}^{-1}$ wet weight. The tissue content of cytoplasmic FABP was measured with enzyme-linked immuno-sorbent assay (15).

\subsubsection{Statistics}

Data are expressed as mean \pm standard deviation. A two-way ANOVA test was used to evaluate torque parameters during a single contraction and content of highenergy phosphates and related compounds. Mice type ( $\mathrm{CK}^{-/}$and wild type) and muscle complex type (plantar and dorsal flexors) were used as between-subject factors. Possible differences in mass parameters, MyHC isoform composition and the oxidative markers between both wild type and $\mathrm{CK}^{-1-}$ mice were analysed with a MannWhitney $U$ test. Moreover, differences in relative torque during the series of 12 consecutive contractions between plantar and dorsal flexors were analysed via a repeated two-way ANOVA with a Tukey post-hoc analysis. Differences were considered significant if $\mathrm{p}<0.05$. Statistical analyses were performed using SPSS 10.0 (SPSS Benelux, Gorinchem, The Netherlands).

\section{4 - Results}

Body mass of $\mathrm{CK}^{-1-}$ mice was approximately $10 \%$ lower than wild type. Both muscle complexes of $\mathrm{CK}^{-/}$mice showed a decrease in mass compared to corresponding wild type muscles. The decrease in dorsal muscle mass $(14 \%)$ of $\mathrm{CK}^{-1-}$ was smaller than the decrease in the mass of the plantar muscle complex (37\%) (table 7.1).

table 7.1 Body and muscle mass of wild type and $\mathrm{CK}^{-1-}$ mice.

\begin{tabular}{lll}
\hline & wild type & $\mathrm{CK}^{-{ }^{*}}$ \\
\cline { 2 - 3 } & & \\
body mass, $g$ & $23.9 \pm 1.2$ & $21.5 \pm 1.7^{*}$ \\
plantar flexors, $m g$ & $150.7 \pm 12.6$ & $95.2 \pm 19.0^{*}$ \\
dorsal flexors, $m g$ & $61.1 \pm 10.3$ & $52.5 \pm 6.1^{*}$ \\
\hline
\end{tabular}

Values are presented as mean $\pm s d$. Statistical differences between wild type $(n=8)$ and $\mathrm{CK}^{-/ .}(\mathrm{n}=8)$ mice are indicated by $*(\mathrm{p}<0.05)$. 
figure 7.1 Representative patterns of torque $(\mathrm{T})$ developed during a single isometric tetanic contraction of the plantar (A) and dorsal flexors (B) of wild type and $\mathrm{CK}^{-/-}$mice.

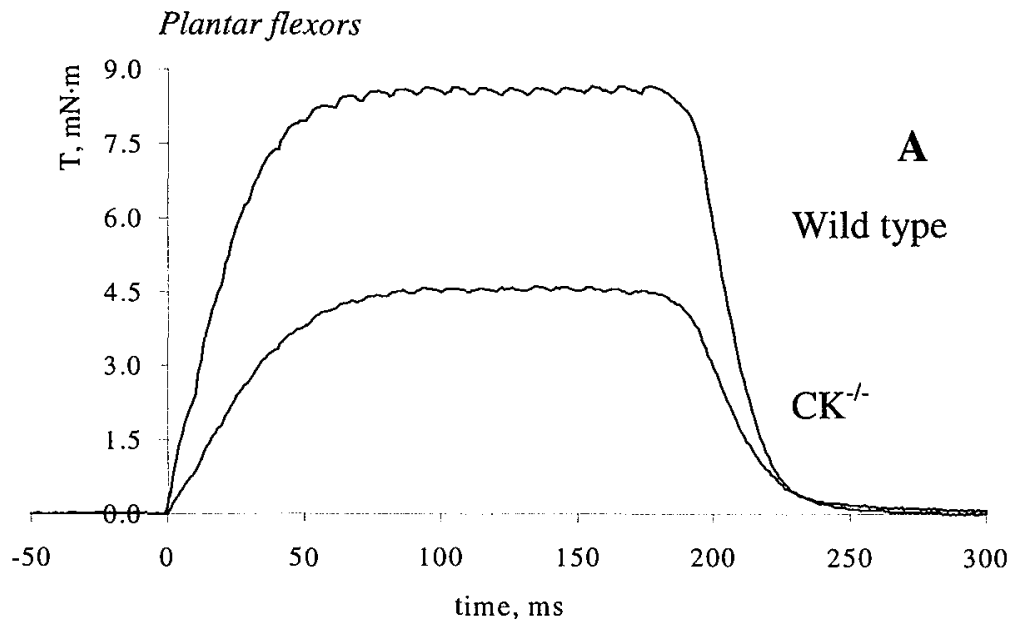

Dorsal flexors

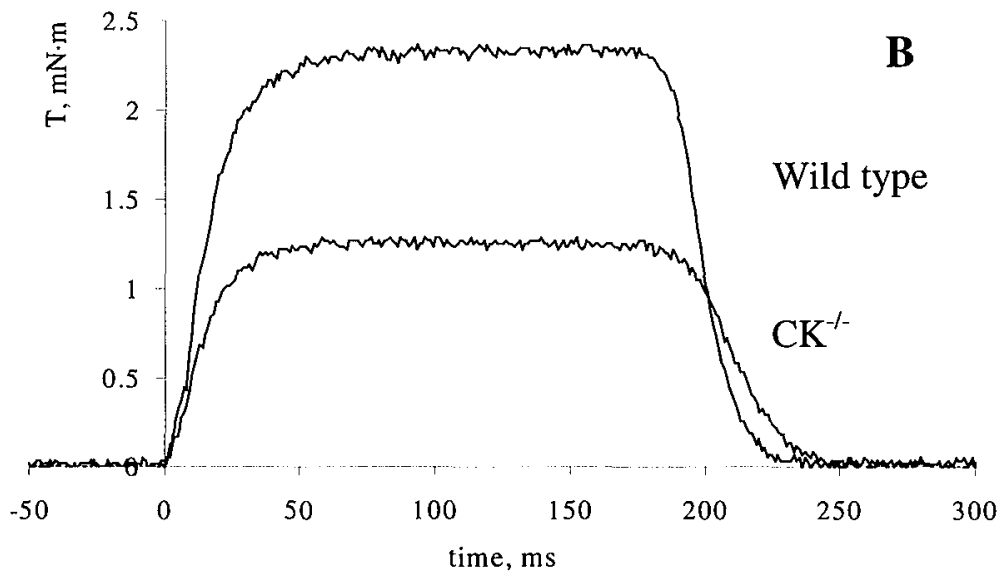

Plantar flexors were stimulated with $100 \mathrm{~Hz}$ and dorsal flexors with a $125 \mathrm{~Hz}$ stimulation frequency. Electrical stimulation started at $0 \mathrm{~ms}$ and was switched of at $170 \mathrm{~ms}$.

Figure 7.1 shows the representative profiles of maximal plantar and dorsal tetanic torque during a single isometric tetanic contraction in wild type and $\mathrm{CK}^{-/-}$mice. Both plantar and dorsal flexors of $\mathrm{CK}^{-/}$mice showed a large decrease in maximal torque. The decrease in maximal tetanic torque of both muscle complexes can partly be explained by the decline in muscle mass in $\mathrm{CK}^{-1-}$ mice (table 7.1). The maximal torque normalised on muscle mass was still lower in $\mathrm{CK}^{-1 /}$ mice than in wild type. 
The developed torque per unit mass was higher in plantar than in dorsal flexors. Since the interaction was not significant, the conclusion was drawn that the decline in torque per unit mass was comparable in both muscle complexes. Furthermore, $\mathrm{CK}^{-1-}$ muscles showed an increase in rise and relaxation time compared to wild type mice. The rise and relaxation time was higher in plantar than in dorsal flexors. No differences in increase in relaxation time between $\mathrm{CK}^{-1}$ plantar and dorsal flexors was found, whereas the rise time was only increased in plantar flexors of $\mathrm{CK}^{-1-}$ mice.

table 7.2 Torque characteristics of a single isometric contraction of plantar and dorsal flexors in wild type and $\mathrm{CK}^{-/}$mice.

\begin{tabular}{|c|c|c|c|c|c|c|c|}
\hline & \multicolumn{2}{|c|}{ plantar flexors } & \multicolumn{2}{|c|}{ dorsal flexors } & \multicolumn{3}{|c|}{ ANOVA } \\
\hline & wild type & $\mathrm{CK}^{\%}$ & wild type & $\mathrm{CK}^{-\alpha}$ & mouse & muscle & interaction \\
\hline $\mathrm{T}, m N \cdot m$ & $9.4 \pm 1.4$ & $5.1 \pm 0.9$ & $2.0 \pm 0.2$ & $1.2 \pm 0.2$ & * & $*$ & ns \\
\hline $\begin{array}{l}\text { T per muscle mass, } \\
m N \cdot m \cdot g^{-l} \text { wet weight }\end{array}$ & $75.2 \pm 13.0$ & $50.8 \pm 10.0$ & $30.1 \pm 5.1$ & $23.8 \pm 4.9$ & $*$ & * & ns \\
\hline $10-50 \%$ rise time, $m s$ & $15.9 \pm 2.1$ & $20.0 \pm 3.2$ & $11.2 \pm 1.3$ & $11.0 \pm 1.3$ & * & * & * \\
\hline $1 / 2$-relaxation time, $m s$ & $13.5 \pm 3.9$ & $16.6 \pm 4.6$ & $11.1 \pm 3.2$ & $14.4 \pm 3.2$ & * & * & ns \\
\hline
\end{tabular}

Plantar flexors were stimulated at $100 \mathrm{~Hz}$ and dorsal flexors were stimulated at $125 \mathrm{~Hz}$ stimulation frequency ( $\mathrm{n}$ $=8$ in each group). Total duration of the tetanic contraction amounted to $170 \mathrm{~ms}$. Data are presented as mean \pm SD. Statistical differences are indicated by $*(p<0.05)$. ns refers to no significant difference. Mouse and muscle in the two-way ANOVA analysis are the between subject factors.

A representative torque output during a series of 12 contractions of both plantar (A) and dorsal (B) flexors in wild type and $\mathrm{CK}^{-1}$ mice are given in figure 7.2. In figure 7.3 the mean and SD of 6 individual experiments is shown. 
figure 7.2 Representative patterns of plantar (A) and dorsal (B) flexor torque (T) developed during a series of 12 tetanic isometric contractions in wild type and $\mathrm{CK}^{-/-}$mice.

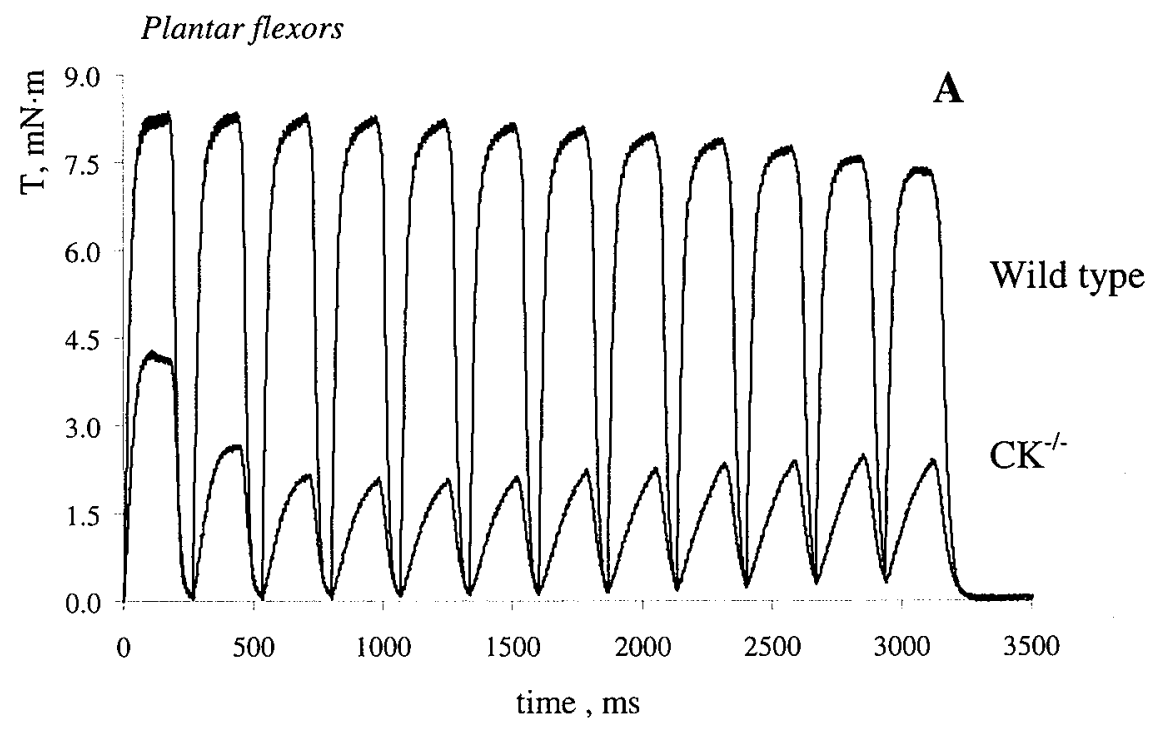

Dorsal flexors

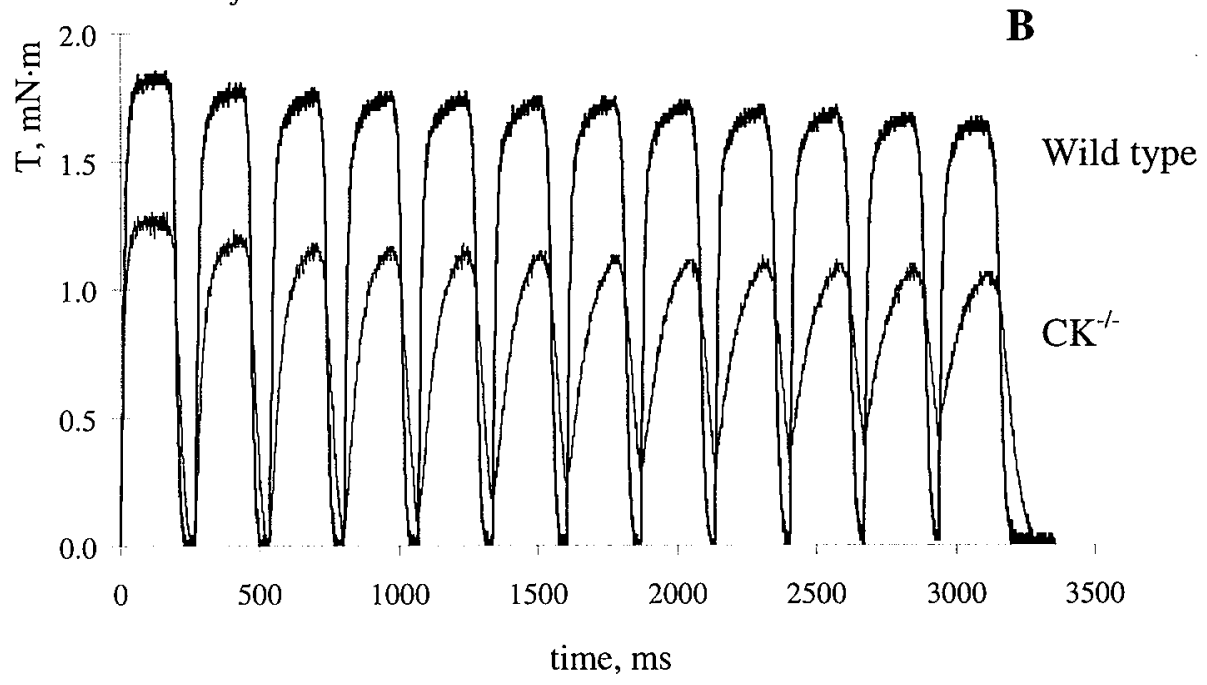

Muscles were supra-maximally stimulated every $265 \mathrm{~ms}$ for $170 \mathrm{~ms}$ with $100 \mathrm{~Hz}$ (plantar flexors) or $125 \mathrm{~Hz}$ (dorsal flexors). 
figure 7.3 Relative plantar and dorsal flexor torque during 12 tetanic isometric contractions in wild type and $\mathrm{CK}^{-/-}$mice.

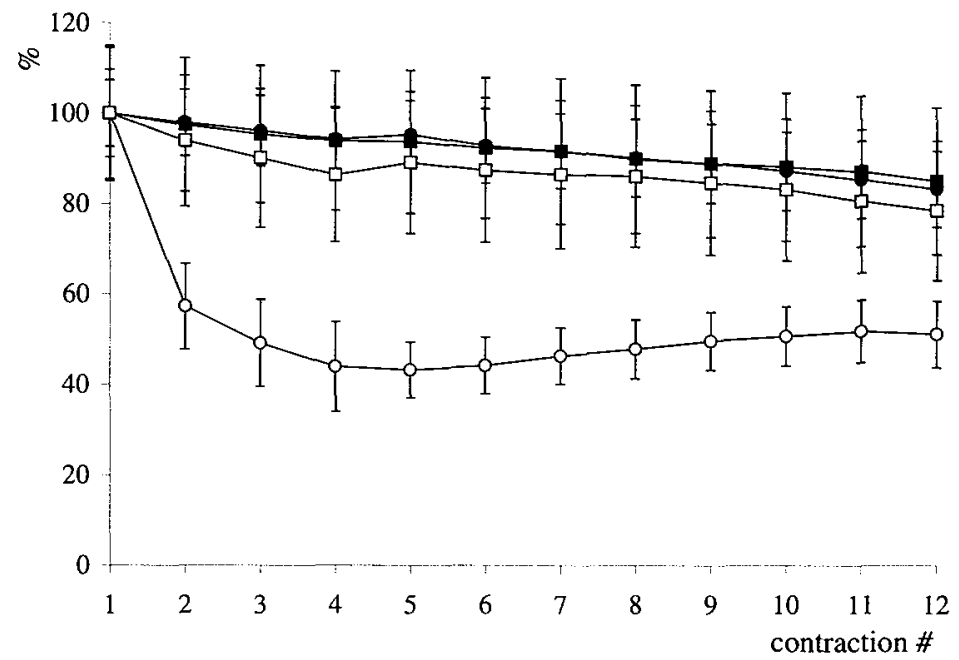

Torque data were expressed as percentage of the torque developed during the first contraction, and refer to mean $\pm \mathrm{sd}\left(\mathrm{n}=8\right.$ in each group). Symbols $\bullet, \mathrm{O}, \square, \square$ refer to wild type plantar flexors, $\mathrm{CK}^{\prime-}$ plantar flexors, wild type dorsal flexors, and $\mathrm{CK}^{-1-}$ dorsal flexors, respectively.

The decline in torque at the $12^{\text {th }}$ contraction and the course of decline in torque during the contraction series were comparable for wild type plantar and dorsal flexors, i.e., the decline amounted to $17 \pm 4 \%$ and $15 \pm 3 \%$, respectively. For both the plantar and dorsal flexors of $\mathrm{CK}$ deficient mice, the relative decline in torque during the $12^{\text {th }}$ contraction was significantly larger compared to wild type mice. The $\mathrm{CK}^{-1-}$ dorsal flexor complex showed gradual decline of $22 \pm 6 \%$ during the course of 12 contractions. In contrast, the plantar flexor complex of $\mathrm{CK}^{-/ .}$mice showed a significantly larger decrease in torque of $43 \pm 11 \%$ at the second and $49 \pm 13 \%$ at the $12^{\text {th }}$ contraction compared to $\mathrm{CK}^{-/-}$dorsal flexors.

Relaxation time of the $12^{\text {th }}$ contraction of wild type plantar and dorsal flexors amounted to $18.8 \pm 4.4$ and $12.9 \pm 3.4 \mathrm{~ms}$, respectively. The relaxation time of the $12^{\text {th }}$ contraction was significantly longer in $\mathrm{CK}^{-1-}$ than in wild type mice, and it was longer in plantar than in dorsal flexors. No interaction was observed indicating that $\mathrm{CK}$ ablation had a similar effect on the relaxation time of the $12^{\text {th }}$ contraction between $\mathrm{CK}^{-/-}$plantar flexors $(24.9 \pm 3.5 \mathrm{~ms})$ and dorsal flexors $(26.2 \pm 2.8 \mathrm{~ms})$.

Table 7.3 shows the basal content of high-energy phosphates and related compounds in resting plantar and dorsal flexor complexes of both wild type and $\mathrm{CK}^{-1-}$ mice. Phosphocreatine ( $\mathrm{PCr}$ ) and creatine ( $\mathrm{Cr}$ ) levels showed no differences between $\mathrm{CK}^{-1-}$ and wild type mice, and between plantar and dorsal flexors. 
Resting ATP values in $\mathrm{CK}^{-1}$ mice were significantly lower than in wild type. The ATP content did not significantly differ between the plantar and dorsal flexors. No interaction was found, indicating that no differences in decline in ATP content present observed between $\mathrm{CK}^{-/}$plantar $(-18 \%)$ and dorsal $(-26 \%)$ flexors. The ADP and AMP content was significantly higher in $\mathrm{CK}^{-/}$than in wild type mice, but did not differ between plantar and dorsal flexors. Furthermore, resting muscle IMP levels exhibited a fourfold higher value in $\mathrm{CK}^{-1}$ than in wild type mice, whereas no differences existed between plantar and dorsal flexors. For the latter metabolites, no significant interactions between mice type and muscle complex type were present.

table 7.3 The content of high-energy phosphates and related compounds in resting plantar and dorsal flexors of wild type and $\mathrm{CK}^{-1-}$ mice.

\begin{tabular}{|c|c|c|c|c|c|c|c|}
\hline & \multicolumn{2}{|c|}{ plantar flexors } & \multicolumn{2}{|c|}{ dorsal flexors } & \multicolumn{3}{|c|}{ ANOVA } \\
\hline & wild type & $\mathrm{CK}^{--}$ & wild type & $\mathrm{CK}^{-1}$ & mouse & muscle & interaction \\
\hline ATP & $30.4 \pm 3.3$ & $24.9 \pm 2.6$ & $28.4 \pm 2.1$ & $20.9 \pm 1.4$ & $*$ & ns & ns \\
\hline ADP & $4.2 \pm 0.4$ & $5.2 \pm 1.1$ & $4.1 \pm 0.5$ & $5.6 \pm 1.2$ & * & ns & ns \\
\hline AMP & $0.2 \pm 0.1$ & $0.4 \pm 0.1$ & $0.2 \pm 0.1$ & $0.6 \pm 0.2$ & $*$ & ns & ns \\
\hline TAN & $34.8 \pm 3.5$ & $30.5 \pm 3.4$ & $32.6 \pm 2.4$ & $26.3 \pm 1.7$ & $*$ & ns & ns \\
\hline IMP & $0.2 \pm 0.1$ & $0.9 \pm 0.6$ & $0.3 \pm 0.1$ & $1.2 \pm 0.5$ & $*$ & ns & ns \\
\hline $\mathrm{PCr}$ & $82.9 \pm 8.7$ & $78.6 \pm 5.7$ & $73.1 \pm 5.3$ & $69.3 \pm 6.6$ & ns & ns & ns \\
\hline $\mathrm{Cr}$ & $42.7 \pm 6.8$ & $42.5 \pm 3.4$ & $46.6 \pm 4.6$ & $42.6 \pm 5.2$ & ns & ns & ns \\
\hline $\mathrm{PCr}+\mathrm{Cr}$ & $125.6 \pm 10.0$ & $121.1 \pm 8.7$ & $120.7 \pm 6.6$ & $111.9 \pm 5.2$ & ns & ns & ns \\
\hline $\mathrm{PCr} / \mathrm{Cr}$ & $2.0 \pm 0.4$ & $1.9 \pm 0.1$ & $1.6 \pm 0.1$ & $1.6 \pm 0.1$ & ns & $*$ & ns \\
\hline
\end{tabular}

Data are expressed as mean and $\mathrm{SD}$ in $\mu \mathrm{mol} \cdot \mathrm{g}^{-1}$ dry weight ( $\mathrm{n}=8$ in each group). The ratio phosphocreatine/creatine $(\mathrm{PCr} / \mathrm{Cr})$ is dimensionless. Total adenine nucleotide content (TAN) was computed as the sum of ATP, ADP, and AMP. Statistical differences are indicated by * $(\mathrm{P}<0.05)$, and no significant difference are indicated by ns. Mouse and muscle in the two-way ANOVA analysis are the between subject factors.

figure 7.4 Relative myosin heavy chain distribution in dorsal and plantar flexors from $\mathrm{CK}^{-1}$ and wild type mice.

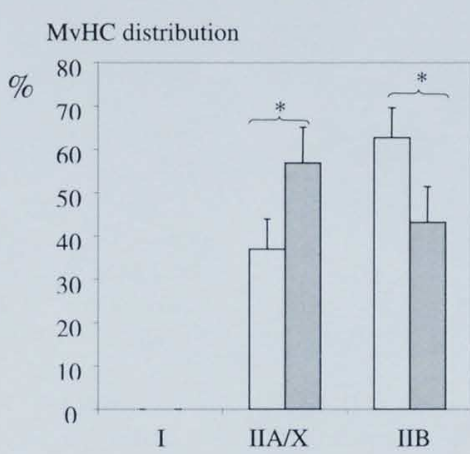

tibialis anterior + EDL muscles

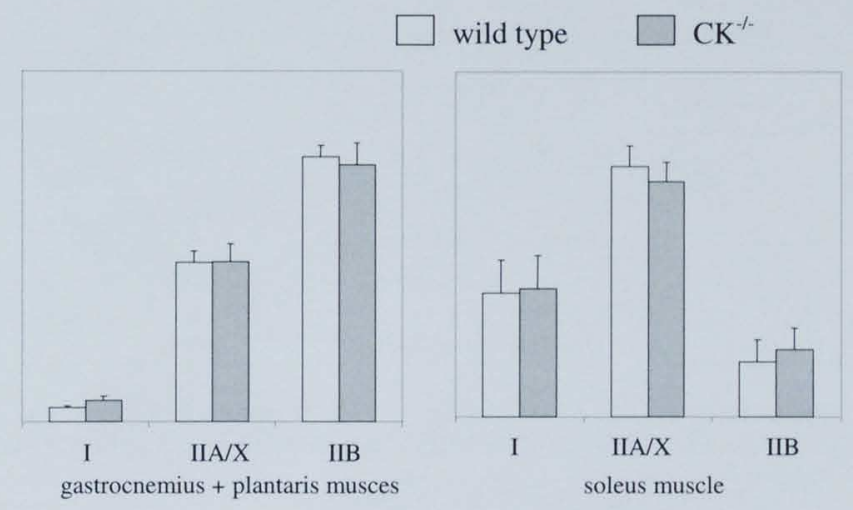

Dorsal flexors

Plantar flexors

Data refer to mean $\pm s d\left(n=4\right.$ in each group). Statistical differences between wild type and $\mathrm{CK}^{-1}$ mice are indicated by * $(\mathrm{p}<0.05)$. 
The tibialis anterior plus EDL muscles, being components of the dorsal flexors, of $\mathrm{CK}^{-1-}$ mice showed a significant shift from type IIB to IIA MyHC isoforms compared to the corresponding wild type muscles (fig. 7.4). The MyHC distribution in the gastrocnemius plus plantaris muscles and in the soleus muscles (plantar flexor complex) did not differ between wild type and $\mathrm{CK}^{-1-}$ mice.

The maximal activity of total creatine kinase in $\mathrm{CK}^{\mu}$ gastrocnemius muscle and dorsal flexor complex was approximately $0.7 \%$ of that of wild type, whereas the soleus muscle exhibited $3.8 \%$ of that of wild type. No differences between wild type and $\mathrm{CK}^{-/}$muscles in maximal activity of total AMP deaminase were found. The gastrocnemius muscle and dorsal flexor complex of $\mathrm{CK}^{-/}$mice showed a significant increase in activity of citrate synthase (CS) and 3-hydroxyacyl-CoA dehydrogenase (HAD) compared to wild type muscles. In contrast, the soleus muscle exhibited no difference in $\mathrm{CS}$ and HAD activity between wild type and $\mathrm{CK}^{-/}$mice. The FABP content of both the gastrocnemius plus plantaris muscles and dorsal flexors was significantly higher in $\mathrm{CK}^{-1-}$ than in wild type mice (table 7.4).

\section{5 - Discussion}

In the present study, we investigated whether the differences in mechanical performance during a series of repeated isometric contractions between isolated gastrocnemius muscle $(5,6)$ and the intact dorsal flexors studied in their natural environment (chapters 5 and 6) were caused by distinct properties of the muscles studied or originated by differences in experimental set-up.

table 7.4 Maximal activity of total creatine kinase (CK), AMP deaminase (AMP-d), citrate synthase (CS), and 3-hydroxyacyl-CoA dehydrogenase (HAD) and the content of FABP in gastrocnemius plus plantaris muscles, soleus muscle (plantar flexors) and tibialis anterior plus $\mathrm{EDL}$ muscles (dorsal flexors) of wild type and $\mathrm{CK}^{-1-}$ mice.

\begin{tabular}{|c|c|c|c|c|c|c|}
\hline & \multicolumn{2}{|c|}{$\begin{array}{l}\text { gastrocnemius plus plantaris } \\
\text { muscles }\end{array}$} & \multicolumn{2}{|c|}{ soleus muscle } & \multicolumn{2}{|c|}{$\begin{array}{l}\text { tibialis anterior plus } \\
\text { EDL muscles }\end{array}$} \\
\hline & wild type & $\mathrm{CK}^{-1-}$ & wild type & $\mathrm{CK}^{-1}$ & wild type & $\mathrm{CK}^{/ /}$ \\
\hline CK & $6520 \pm 910$ & $30 \pm 10 * *$ & $2900 \pm 750$ & $110 \pm 80 * *$ & $5670 \pm 830$ & $37 \pm 9 * *$ \\
\hline AMP-d & $60.7 \pm 12.7$ & $64.7 \pm 10.2$ & - & - & $51.6 \pm 14.5$ & $48.4 \pm 11.1$ \\
\hline $\mathrm{CS}$ & $231 \pm 86$ & $364 \pm 50 *$ & $366 \pm 89$ & $382 \pm 122$ & $309 \pm 55$ & $414 \pm 84 *$ \\
\hline HAD & $600 \pm 180$ & $1060 \pm 270 *$ & $1220 \pm 390$ & $1140 \pm 420$ & $860 \pm 160$ & $1440 \pm 260 *$ \\
\hline FABP & $0.07 \pm 0.04$ & $0.13 \pm 0.03 *$ & - & - & $0.03 \pm 0.02$ & $0.10 \pm 0.02 *$ \\
\hline
\end{tabular}

Values refer to mean and SD ( $n=6$ for each group). Enzyme activity is expressed as $U \cdot \mathrm{mg}^{-1}$, except for AMP-d $\left(\mathrm{U} \cdot \mathrm{g}^{-1}\right)$, and FABP as $\mathrm{mg} \cdot \mathrm{g}^{-1}$. Statistical differences between wild type and $\mathrm{CK}^{-1}$ are indicated by $*(\mathrm{p}<0.05)$ and $* *(\mathrm{p}<0.01)$. 
For this purpose, we assessed the consequence of $\mathrm{CK}$ deficiency on mechanical performance in the intact plantar and dorsal flexors. The present findings showed that the intact plantar flexors of $\mathrm{CK}^{-/}$mice also exhibited a fast and substantial decline in torque development during a series of consecutive contractions, as has been found in the isolated gastrocnemius muscle. This phenomenon did, however, not occur in the intact dorsal flexors of $\mathrm{CK}^{-1 /}$ mice. These findings indicate that the differences between the plantar and dorsal flexors are not related to differences in experimental set-up, i.e., isolated muscle vs. intact muscle complex, but most likely due to distinct properties of the muscle complexes under investigation.

The maximal tetanic torque per unit muscle mass during a single tetanic contraction was lower in $\mathrm{CK}^{-\gamma_{-}}$mice than in wild type (table 7.2), confirming previous observations on force development in isolated muscles from $\mathrm{CK}^{-1}$ mice $(5,6,16)$. Data from chapter 6 showed that elevated IMP and low ATP content do not exert the negative effect on muscle contractile behavior under the present experimental conditions. Moreover, the absolute torque per unit muscle mass was substantially larger in plantar flexors than in dorsal flexors. Differences in muscular torque can be caused, among others, by variation in the length of the lever arm since torque equals the product of force and the length of the lever arm. The lengths of lever-arm of the plantar and dorsal flexors were estimated at approximately 2.1 and $1.0 \mathrm{~mm}$, respectively (19). Taking into account these lever arm distances, the maximal isometric force per unit muscle mass of plantar and dorsal flexors of the $\mathrm{CK}^{-/}$mice amounted to $24.2 \pm 4.8$ and $23.8 \pm 4.9 \mathrm{~N} \cdot \mathrm{g}^{-1}$, respectively and to $35.8 \pm 6.2$ and $30.1 \pm$ $4.9 \mathrm{~N} \cdot \mathrm{g}^{-1}$ for corresponding wild type muscles. Finally, no interaction for torque per unit muscle mass was found between muscle complex type and wild type and $\mathrm{CK}^{-1-}$ mice, indicating that the negative effect of $\mathrm{CK}$ ablation on torque development is similar in the two muscle complexes under investigation.

The higher rise time in plantar flexors in both wild type and $\mathrm{CK}^{-1-}$ mice can most likely be explained by the lower stimulation frequency used in this muscle complex, i.e., 100 and $125 \mathrm{~Hz}$ for plantar and dorsal flexors, respectively. Moreover, the plantar flexor complex consists of a relatively greater proportion of slow-twitch $\mathrm{MyHC}$ than the dorsal flexor complex. This MyHC isoform is associated with a lower rate of cross-bridge cycling, which might prolong the rise time of the muscle complex. Furthermore, relaxation after a single tetanic contraction was prolonged to a similar extent in both plantar and dorsal flexors of $\mathrm{CK}^{-/}$mice compared to wild type muscles. This points towards a decreased sarcoplasmic reticulum $\mathrm{Ca}^{2+}$-ATPase (SERCA II) activity, which may be directly related to alterations in the energy status of the $\mathrm{CK}^{-1-}$ muscle cell (6). 
When plantar and dorsal flexor muscles of CK deficient mice were challenged by a series of repeated isometric tetanic contractions, interesting differences in timecourse of torque development became apparent. The torque output of the CK deficient plantar flexors exhibited a substantial decline already during the second contraction, as has also been found in isolated muscles $(6,7)$. In contrast, the torque output of the CK deficient dorsal flexors showed a gradual decline, indicating that this muscle complex does not show the phenomenon called 'lack of burst activity'. It could be speculated that the difference in MyHC isoform adaptation might play a role. In this respect, it is of interest to note that in the dorsal flexors, which showed a gradual decline in torque, exhibited a significant shift from the IIB MyHC isoform to the slower IIA/X isoform. This adaptational change was not observed in the $\mathrm{CK}^{-1}$ plantar flexors, which could not sustain a series of repeated high-intensity contractions. Despite the intriguing relationship between muscular performance and adaptations in $\mathrm{MyHC}$, at present the precise cause of the difference in mechanical behaviour between $\mathrm{CK}^{-/-}$plantar and dorsal flexors is still incompletely understood.

Assessment of the maximal activity of oxidative marker enzymes revealed that both the dorsal flexor complex and the gastrocnemius muscle showed an increase in maximal activity of the mitochondrial marker enzyme citrate synthase (CS). Moreover, the increase in the tissue content of the cytoplasmic fatty acid-binding protein (FABP), required for the transport of fatty acids from the sarcolemma to the mitochondrial site of conversion, and the enhanced maximal activity of hydroxyacyl-CoA dehydrogenase (HAD), a key enzyme in the $\beta$-oxidation pathway, indicate that the capacity of the $\mathrm{CK}^{-/-}$muscle complexes to utilise fatty acids is augmented. In contrast, the soleus muscle, a typical slow twitch oxidative muscle, did not respond to CK deficiency by increasing the maximal activity of CS and HAD. The conclusion, therefore, can be drawn that glycolytic muscles differently react to $\mathrm{CK}$ deficiency than oxidative muscles. It is, however, unlikely that these adaptations do play a role in energy conversion in the present contraction series, since the muscle complexes were forced to perform isometric contractions of high-intensity with a tour duty cycle of 0.64 . The adaptations in mitochondrial density and capacity to oxidise fatty acids could be beneficial when the muscle complex is subjected to a contraction series of long duration and low-intensity. Under the latter conditions, oxidative metabolism will prevail over anaerobic ATP generation.

In conclusion, during a series of repeated high-intensity contractions, the relative torque of the $\mathrm{CK}$ deficient plantar flexors exhibited a substantial decline during the second contraction, which contrasted with the gradual decline in relative torque observed in the dorsal flexors of $\mathrm{CK}^{-/}$. 
The dorsal flexors also showed a significant shift of the type IIB MyHC isoform towards the slower type IIA $/ \mathrm{X}$ isoform, which was not observed in the $\mathrm{CK}^{-1-}$ plantar flexors. Despite this relationship, the exact cause of the differences in mechanical performance between $\mathrm{CK}^{-1-}$ plantar and dorsal flexors remains to be elucidated.

\section{6 - Acknowledgement}

The authors thank Prof. Dr. B. Wieringa (Department of Cell Biology and Histology, University of Nijmegen, The Netherlands) for kindly providing us the CK knockout mice and wild type littermates. Furthermore, the authors thank Dr. J. F. C. Glatz (Department of Physiology, University Maastricht, The Netherlands) for his help in analysing the tissue content of FABP, and thank Dr. J. J. Adam (Department of Movement Sciences, University Maastricht, The Netherlands) for his help in the statistical analysis. 


\section{7 - References}

1. Gorselink, M., Drost, M. R., Louw de, J., Willems, P. J. B., Rosielle, N., Janssen, J. D., and Van der Vusse, G. J. (2000) Accurate assessment of in situ isometric contractile properties of hind limb plantar and dorsal flexor muscle complex of intact mice. Pflügers Arch. 439, 665-670

2. Wyss, M., and Walliman, T. (1994) Creatine metabolism and the consequences of creatine depletion in muscle. Mol. Cell. Biochem. 133/134, 51-66

3. Wyss, M., Smeitink, J., Wevers, R. A., and Walliman, T. (1992) Mitochondrial creatine kinase: a key enzyme of aerobic energy metabolism. Biochim. Biophys. Acta $1102,119-166$

4. Bessman, S. P., and Geiger, P. J. (1981) Transport of energy in muscle: the phosphorylcreatine shuttle. Science $211,448-452$

5. Steeghs, K., Oerlemans, F., de Haan, A., Heerschap, A., Verdoodt, L., de Bie, M., Ruitenbeek, W., Benders, A., Jost, C., van Deursen, J., Tullson, P., Terjung, R., Jap, P., Jacob, W., Pette, D., and Wieringa, B. (1998) Cytoarchitectural and metabolic adaptations in muscles with mitochondrial and cytosolic creatine kinase deficiencies. Mol. Cell Biochem. 184, 183-194

6. Steeghs, K., Benders, A., Oerlemans, F., de Haan, A., Heerschap, A., Ruitenbeek, W., Jost, C., dan Deursen, J., Perryman, B., Pette, D., Bruckwilder, M., Koudijs, J., Jap, P., Veerkamp, J., and Wieringa, B. (1997) Altered $\mathrm{Ca}^{2+}$ responses in muscles with combined mitochondrial and cytosolic creatine kinase deficiencies. Cell 89, 93-103

7. van Deursen, J., Heerschap, A., Oerlemans, F., Ruitenbeek, W., Jap, P., ter Laak, H., and Wieringa, B. (1993) Skeletal muscles of mice deficient in muscle creatine kinase lack burst activity. Cell 74, 621-631

8. Gorselink, M., Drost, M. R., Coumans, W. A., Kranenburg van, G. P. J., Hesselink, R. P., and van der Vusse, G. J. Impaired muscular contractile perfromance and adenine nucleotide handling in creatine kinase deficient mice. Am. J. Physiol. under revision

9. Brooks, S. V., and Faulkner, J. A. (1991) Forces and powers of slow and fast skeletal muscles in mice during repeated contractions. J. Physiol. 436, 701-710

10. Fitts, R. H. (1994) Cellular mechanisms of muscle fatigue. Physiol. Rev. 74, 49-94

11. Edwards, R. H. T., Hill, D. K., and Jones, D. A. (1975) Metabolic changes associated with the slowing of relaxation in fatigued mouse muscle. J. Physiol. 251, 287-301

12. Van der Vusse, G. J., Coumans, W. A., Van der Veen, F. H., Drake, A., Flameng, W., and Suy, R. (1984) ATP, creatine phosphate and glycogen content in human myocardial biopsies: markers for the efficacy of cardioprotection. Vasc. Surg. 8, 127134

13. Degens, H., Yu, F., Li, X., and Larsson, L. (1998) Effects of age and gender on shortening velocity and myosin isoforms in single rat muscle fibres. Acta Physiol Scand $163,33-40$

14. Rundell, K. W., Tullson, P. C., and Terjung, R. L. (1992) Altered kinetics of AMP deaminase by myosin binding. Am. J. Physiol. 263, C294-299

15. Vork, M. M., Glatz, J. F., Surtel, D. A., Knubben, H. J., and Van der Vusse, G. J. (1991) A sandwich enzyme linked immuno-sorbent assay for the determination of rat heart fatty acid-binding protein using the streptavidin-biotin system. Application to tissue and effluent samples from normoxic rat heart perfusion. Biochim Biophys Acta 1075, 199-205

16. Dahlstedt, A. J., Katz, A., Wieringa, B., and Westerblad, H. (2000) Is creatine kinase responsible for fatigue? Studies of isolated skeletal muscle deficient in creatine kinase. FASEB J. 14, 982-990 
17. Westerblad, H., Allen, D. G., Bruton, J. D., Andrade, F. H., and Lannergren, J. (1998) Mechanisms underlying the reduction of isometric force in skeletal muscle. Acta Physiol. Scand. 162, 253-260

18. Westra, H. G., de Haan de, A., van Doorn van, J. E., and de Haan, J. (1990) IMP production and energy metabolism during exercise in rats in relation to age. Biochem. J. 239, 751-755

19. Ashton-Miller, J. A., Veerichetty, Y. H. E., and Kadhiresan, A. (1992) An apparatus to measure in vivo biomechanical behavior of dorsi- and plantarflexors of mouse ankle. Pflügers Arch. 72(3), 1205-1211 
Chapter 7

120 


\section{Chapter 8}

\section{I NCREASED MUSCLE FATIGABILITY IN GLUT4- DEFICIENT MICE.}

M. Gorselink, M.R. Drost, K.F.J. de Brouwer, G. Schaart, G.P.J. van Kranenburg, T.H.M. Roemen, M. van Bilsen, M. J. Charron, and G.J. van der Vusse (2001) Am. J. Physiol, submitted.

Description of GLUT4 deficiency on isometric and shortening contractile performance of the dorsal flexor muscle complex in situ. Moreover, we have explored the hypothesis that lack of GLUT4 enhances muscular fatigability. 


\section{1 - Summary}

GLUT4 plays a predominant role in glucose uptake during muscular contraction. In the present study, we have investigated in mice whether disruption of the GLUT4 gene affects isometric and shortening contractile performance of the dorsal flexor muscle complex in situ. Moreover, we have explored the hypothesis that lack of GLUT4 enhances muscular fatigability.

Isometric performance normalised per unit muscle mass during a single tetanic contraction did not differ between wild type and GLUT4 $^{-1-}$ mice. Shortening contractions, however, revealed a significant 1.4 fold decrease in peak power per unit mass, most likely caused by the fibre type transition from fast-glycolytic fibres (IIB) to fast-oxidative fibres (IIA) in GLUT4 ${ }^{-/}$dorsal flexors. Moreover, it can be concluded that GLUT4 is essential for glycogen formation in the dorsal flexor complex since basal glycogen level is substantially decreased in GLUT4 deficient mice. The decreased glycogen level in GLUT4 ${ }^{-/-}$dorsal flexors most likely contributes to increased fatigue susceptibility in GLUT4 ${ }^{-/}$mice. The significant decrease in relative work output during the steady state phase indicates that energy supply through compensatory routes is not capable to fully compensate for the lack of GLUT4. 


\section{2 - Introduction}

Glucose is a major fuel for contracting muscle fibres $(1,2)$. This substance is supplied to the muscle fibre from extra- and intracellular sources, i.e., the blood glucose pool and the intracellular glycogen $(3,4)$. Uptake of glucose by skeletal muscle cells is facilitated by a family of membrane-associated glucose transporters, GLUTs $(1,5)$. Basal glucose uptake is mediated via the GLUT1 isoform, whereas the bulk of glucose uptake is primarily mediated via the insulin- and contraction regulatable glucose transporter GLUT4 (6-10). Following uptake, glucose is metabolised to generate ATP or is stored as glycogen (11). During the initial phase of moderate-intensity exercise, skeletal muscle uses the intracellular glycogen store to meet its energy demand (12). Ryder and co-workers recently showed that during electrical stimulation blood glucose also serves as a suitable substrate during moderate intensity exercise in mice (13). To enable detailed studies on the specific role of GLUT4 in glucose homeostasis in general and in muscular functioning in particular, Katz and colleagues (14) recently generated mice with the GLUT4 gene disrupted $\left(\mathrm{GLUT4}^{-1-}\right)$.

Despite the importance of regulatable glucose transporters for muscular energy metabolism, information on the impact of GLUT4 deficiency on muscle contractile performance is scarce. Ryder and colleagues (13) recently reported that developed isometric tension during in vitro electrical stimulation of isolated extensor digitorum longus (EDL) muscle did not differ between wild type and GLUT4 ${ }^{-1}$ mice. Extrapolation of these findings to the in vivo situation, however, should be done with caution, since the muscle fibres were studied isolated from their natural surroundings, and only isometric contractile function was studied.

In the present study, we have investigated whether disruption of the GLUT4 gene affects muscular function in situ. To this end, we recently developed an experimental model to monitor isometric and shortening parameters of intact mouse skeletal muscle $(15,16)$. These parameters include torque development of the dorsal flexors during and the rate of relaxation after a single isometric tetanic contraction on the one hand, and peak power and optimal and maximal shortening velocity on the other. Mass of the dorsal flexor complex and its fibre composition were assessed to explain possible differences in muscle contractile behaviour between wild type and GLUT $^{-/-}$mice. Moreover, we have explored the hypothesis that lack of GLUT4 results in enhanced fatigability of the dorsal flexor complex subjected to a contraction protocol of moderate intensity. This hypothesis was based on the notion that under normal conditions glucose derived from both the blood plasma and the intracellular glycogen is an important fuel for muscles contracting at moderate intensity $(12,17)$. 
In a subset of experiments, muscular glycogen content was measured prior to and after the contraction protocol.

\section{3 - Methods}

\subsubsection{Animals}

Male mice deficient in GLUT4 as described by Katz et al. (14) were used in the present study. Twelve-week-old male littermates (C57B1/6) served as age-matched controls. Genotyping of the wild type and GLUT4 $4^{-1-}$ mice was performed via PCR analysis. Briefly, isolation of mouse tail DNA was performed with the DNeasy ${ }^{\mathrm{TM}}$ tissue kit (Qiagen, Hilden, Germany) according to the manufacturers instructions. DNA (75 ng) was analysed in a total volume of $25 \mu$ l containing 1xPCR buffer, 0.2 $\mathrm{mM}$ of each dNTP, $1.5 \mathrm{mM} \mathrm{MgCl}, 24 \mathrm{nmol}$ of each oligonucleotide primer and 2.5 Units Taq DNA polymerase, recombinant (Life Technologies, Rockville, MD, USA). Two separate reactions were performed to establish the genotype. Primers against the neomycin cassette (forward primer: 5'-TGACTGGGCACAACAGACAATCGGC-3' and reverse primer: 5'-GTAGCCAA-CGCTATGTCCTGATAGC-3') were used to detect disrupted GLUT4 allele(s). Amplification of this sequence ( 35 cycles of $30 \mathrm{~s}$ at $95^{\circ} \mathrm{C}, 30 \mathrm{~s}$ at $63^{\circ} \mathrm{C}$ and $1 \mathrm{~min}$ at $72^{\circ} \mathrm{C}$ ) resulted in a $650 \mathrm{bp}$ fragment. To detect the (uninterrupted) wild type GLUT4 allele(s) primers were used spanning the target region, i.e., forward primer: 5'-TCTTG-ATGACCGTGGCTCTG-3' in exon 9 and reverse primer: 5'-GCCGACAAT-GAAGTTACAGG-3' in exon 10. For the wild type allele, this resulted in an amplified fragment of $750 \mathrm{bp}\left(35\right.$ cycles of $30 \mathrm{~s}$ at $95^{\circ} \mathrm{C}, 30 \mathrm{~s}$ at $60^{\circ} \mathrm{C}, 1 \mathrm{~min}$ at $72^{\circ} \mathrm{C}$ ).

During the measurements of contractile properties, the animals were anaesthetised with Halothane (Fluothane ${ }^{\circledR}$, Zeneca, Ridderkerk, The Netherlands), supplied in $\mathrm{O}_{2}$ and $\mathrm{N}_{2} \mathrm{O}(3: 1,1.5-2.0 \%)$ via a facemask through a flowmeter system (4.0 $1 \cdot \mathrm{min}^{-1}$; Medec, Montvalle, N.J., USA). All experimental procedures were approved by the Institutional Animal Care and Use Committee of the University Maastricht and complied with the principles of laboratory animal care.

\subsubsection{Surgical procedure}

The anaesthetised mice were positioned on a thermostatic platform $(38.5 \pm 0.1$ ${ }^{\circ} \mathrm{C}$ ). After locally depilating the skin, a small incision in the lateral part of the knee was made to expose the peroneal nerve. A bipolar platinum electrode was carefully attached to the nerve to electrically stimulate the dorsal muscle complex (tibialis anterior muscle and EDL muscle) via a pulse generator (HSE 215/IZ, Freiburg, Germany). The position of the electrode was changed, if a current of more than 1.0 $\mathrm{mA}$ was needed to obtain supra-maximal muscle contraction. 
The electrode was fixated to the skin with cyano-acrylate glue to prevent electrode displacement during the experimental period.

\subsubsection{Experimental model}

For proper determination of isometric contractile properties of the intact dorsal flexor complex, the anaesthetised mouse was fixated via the hip and foot to the measurement device as previously described in detail (15). Supra-maximal stimulation current, necessary to obtain recruitment of all fibres of the muscle complex under investigation, was first assessed using 3 to 5 isometric double-twitch contractions ( 5 ms interval) with increasing current. Resting periods between the double-twitches were kept constant at $60 \mathrm{~s}$. Optimal muscle length at optimal ankle angle was determined using 11 double-twitch contractions at ankle angles between $20^{\circ}$ dorsal flexion and $30^{\circ}$ plantar flexion. All measurements were determined at optimal (isometric contractions) or around (shortening contractions) optimal ankle angle. Maximal tetanic torque was obtained with a $150 \mathrm{~ms}$ pulse train and $125 \mathrm{~Hz}$ stimulation frequency. Torque signals were digitised and analysed for maximal tetanic torque and half-relaxation time. Maximal torque was determined from filtered torque signals (11 point moving-average filter). The 1/2-relaxation time was determined from the unfiltered torque tracings, and was calculated as the time required for torque to decline from 50 to $25 \%$ of its maximal value (18).

Thereafter, the isometric measurement device was replaced by the mouse ergometer, i.e., the device appropriate for measuring the shortening properties of the skeletal muscle contraction (16). During this procedure, the optimal ankle angle was maintained. Muscle shortening velocity was adjusted stepwise via variation in angular stroke and rotation frequency as indicated in table 8.1. Stimulation frequency was adjusted to angular velocity (19). Maximal torque during subsequent shortening contractions was defined as torque at the optimal ankle angle. The torque-velocity relation was fitted to a hyperbola described by the Hill equation (20). Power was determined from the Hill curve, and calculated as the product of angular velocity and torque generated at this angular velocity. Optimal shortening velocity $\left(\mathrm{V}_{\mathrm{opt}}\right)$ was defined as the value of shortening velocity at peak power. Maximal shortening velocity $\left(\mathrm{V}_{\text {max }}\right)$ was determined as the value of the intercept of the Hill curve with the velocity axis. Data acquisition was performed at $1000 \mathrm{~Hz}$ with an Apple Macintosh 7100 PowerPC $^{\circledR}$ with an 8 channel, 12 bits Lab-NB analogue-to-digital conversion board (National Instruments ${ }^{\circledR}$, Mopac Expwy, Austin, Tx, USA) under Labview ${ }^{\circledR} 3.1$. Post-processing of the torque data was performed with Matlab 5.2.1 (The Math Works, Inc., Natick, Ma, USA). 
table 8.1 Input parameters of the stimulation protocol for assessment of the torque-velocity relationship of intact dorsal flexors.

\begin{tabular}{|c|c|c|c|c|c|}
\hline Chronological order of stimulation conditions & $(1)$ & (2) & (3) & (4) & (5) \\
\hline frequency, $\mathrm{Hz}$ & 1 & 8 & 2 & 4 & 4 \\
\hline angular stroke," & 20 & 20 & 20 & 15 & 20 \\
\hline stimulation frequency, $\mathrm{Hz}$ & 125 & 300 & 150 & 200 & 200 \\
\hline stimulation phase, ${ }^{\circ}$ & 145 & 95 & 120 & 105 & 105 \\
\hline stimulation duration, $m s$ & 150 & 30 & 150 & 70 & 70 \\
\hline measured velocity at optimal angle, ${ }^{\circ} \cdot s^{-1}$ & $129 \pm 3$ & $1016 \pm 5$ & $260 \pm 4$ & $388 \pm 5$ & $516 \pm 3$ \\
\hline
\end{tabular}

Last row represents the measured velocity at optimal ankle angle $(n=10)$.

\subsubsection{Fatigue protocol}

Following 10 minutes recovery period after the determination of the basal isometric and shortening properties, dorsal flexors were subjected to a series of 150 shortening contractions. The muscle complex was electrically stimulated at $125 \mathrm{~Hz}$ for $200 \mathrm{~ms}$ every $2000 \mathrm{~ms}$ (tour duty cycle: 0.1 ), at $1 \mathrm{~Hz}$ angular frequency with a $20^{\circ}$ stroke. In this way electrically stimulated and non-stimulated strokes alternated. The torque output measured during electrical stimulation equals the sum of frictional torque, inertial torque, passive muscle torque, and the active muscle torque. The active torque of the muscle contraction was calculated by subtraction of the torque measured during a non-stimulated stroke (frictional torque, inertial torque, and passive torque) from the torque signal measured in a stimulated stroke (total torque). Work output of the individual shortening contractions was calculated by integrating active torque over angle.

\subsubsection{Tissue sampling}

A subset of mice was used to determine glycogen content in the dorsal flexor complex following the 150 shortening contractions. The muscle complex of the nonstimulated contra-lateral leg was used as control. These contra-lateral muscles were also used for analysis of resting high-energy phosphate levels. A second set of contralateral muscles was frozen in melting isopentane for light microscopic analysis, i.e., myosin ATPase staining and cross-sectional areas of the muscle fibres. The tissue samples were stored at $-80^{\circ} \mathrm{C}$ until analysis.

\subsubsection{Biochemical assays}

Prior to glycogen and high-energy phosphate analysis, muscle complexes were freeze-dried overnight at $-30^{\circ} \mathrm{C}$. High-energy phosphates and related compounds were assessed with a HPLC method $(21,22)$. 
Glycogen content was measured fluorimetrically in $\mathrm{HCl}$ extracts of the dorsal flexors and expressed as $\mu \mathrm{mol}$ glycosyl units $\cdot \mathrm{g}^{-1}$ dry weight (23). For fibre type distribution and fibre diameter analysis, the mid-belly regions of the dorsal flexor complex were frozen in melting isopentane. Frozen dorsal flexors were cryosectioned $(10 \mu \mathrm{m})$ at -20 ${ }^{\circ} \mathrm{C}$, and thaw mounted on glass slides and air-dried until analysis for myosin ATPase staining (24) and fibre diameter measurements. Briefly, sections were immersed in acetate buffer at $\mathrm{pH} 4.5$, washed in TRIS buffer ( $\mathrm{pH} 7.8$ ), and subsequently rinsed in distilled water. Sections were incubated in fresh ATP-glycine buffer $(\mathrm{pH} 9.4)$, quenched in $\mathrm{CaCl}_{2}(1 \%)$, washed in distilled water and incubated in $\mathrm{CoCl}_{2}(1 \%)$. Thereafter, the sections were incubated in ammonium-sulfite and rinsed in distilled water. Slides are dehydrated in ethanol-xylol series, and embedded in enthallan. Sections were photographed microscopically (Leica, Rijswijk, The Netherlands) and a distinction was made between the tibialis anterior muscle and EDL muscle. Hundred fibres were counted for each muscle, and fibres were classified as a type I, IIA, and IIB fibres (24). Fibres were classified miscellaneous when no differentiation could be made between IIA and IIB fibres. Cross-sectional area of the distinctive fibres was determined from the smallest diameter, assuming ellipsoid cross-sections.

\subsubsection{Statistics}

Data are expressed as mean and standard deviation. Differences in torque parameters, high-energy phosphates, glycogen content, and fibre characteristics between GLUT4 deficient mice and wild type littermates were analysed using nonparametric Mann-Whitney $U$ test. Differences in work output during the exercise protocol were analysed using repeated measures one-way ANOVA with Tukey posthoc analyses to identify differences between GLUT4 deficient and wild type mice. Differences were considered significant if $\mathrm{p}<0.05$. SPSS 9.0 (SPSS Benelux, Gorinchem, The Netherlands) was used for statistical analyses.

\section{4 - Results}

The values of body mass, heart mass, and dorsal flexor complex mass are shown in table 8.2. Body mass of GLUT4 $4^{-/}$mice was significantly lower than agematched littermates $(\mathrm{p}<0.05)$. Dorsal flexor mass of GLUT4 ${ }^{-/}$mice amounted to 59.2 $\pm 5.8 \mathrm{mg}$, which was significantly lower than the dorsal flexors of the littermates $(69.3$ $\pm 9.7 \mathrm{mg}$ ). In contrast, heart mass of the $\mathrm{GLUT}^{-1-}$ mice showed a 1.4 fold increase compared to the wild type $(\mathrm{p}<0.05)$. 
table 8.2 Mass characteristics of wild type and GLUT4 ${ }^{\%}$ mice.

\begin{tabular}{lcc}
\hline \hline & wild type & GLUT4 ${ }^{--}$ \\
\cline { 2 - 3 } & & \\
body mass, $g$ & $26.2 \pm 2.0$ & $22.5 \pm 1.2 *$ \\
dorsal flexor mass, $m g$ & $69.3 \pm 9.7$ & $59.2 \pm 5.8^{*}$ \\
heart mass, $m g$ & $120 \pm 11$ & $165 \pm 16^{*}$ \\
\hline
\end{tabular}

Data refer to mean and SD ( $\mathrm{n}=10$ in each group). Statistical differences between wild type and GLUT4 ${ }^{\%}$ are indicated by $*(\mathrm{p}<0.05)$.

\subsubsection{Single isometric and shortening contraction}

Maximal torque developed during a single isometric tetanic contraction of the dorsal flexors at $125 \mathrm{~Hz}$ stimulation frequency was significantly higher in wild type littermates than in GLUT4 ${ }^{-1-}$ mice by a factor of 1.3 (table 8.3). However, when the maximal torque was normalised on dorsal flexor mass, the values of GLUT4 $4^{-1-}$ mice did not differ from their wild type littermates. Half relaxation time after a maximal tetanic contraction was not significantly different between GLUT4$^{-1 /}$ and wild type muscles.

Shortening properties of the dorsal flexor muscle complex were characterised in terms of peak power, optimal shortening velocity and maximal shortening velocity (table 8.3). The absolute value of peak power was significantly reduced in GLUT4 ${ }^{-1-}$ as compared to the wild type muscle complex. Even after normalisation on dorsal flexor muscle mass the difference between GLUT4 $^{-1-}$ and wild type muscles remained statistically significant. Optimal and maximal shortening velocity of the dorsal flexor muscle complex did not differ between GLUT4 ${ }^{-/-}$and wild type littermates.

table 8.3 Torque characteristics of a single isometric contraction and of shortening contractions of intact dorsal flexors of wild type and GLUT4 ${ }^{-1-}$ mice.

\begin{tabular}{|c|c|c|}
\hline & wild type & GLUT4 ${ }^{\mu-}$ \\
\hline \multicolumn{3}{|l|}{ isometric contraction } \\
\hline torque, $m N \cdot m$ & $2.2 \pm 0.4$ & $1.7 \pm 0.2 *$ \\
\hline torque per unit muscle mass, $m N \cdot m \cdot g^{-l}$ & $31.7 \pm 5.6$ & $29.0 \pm 4.0$ \\
\hline $1 / 2$-relaxation time, $m s$ & $8.6 \pm 1.6$ & $9.7 \pm 1.2$ \\
\hline \multicolumn{3}{|l|}{ shortening contractions } \\
\hline peak power per unit muscle mass, $W \cdot g^{-I}$ & $0.36 \pm 0.09$ & $0.26 \pm 0.01 *$ \\
\hline $\mathrm{V}_{\mathrm{opt}}, " \mathrm{~s}^{-1}$ & $1330 \pm 300$ & $1120 \pm 310$ \\
\hline $\mathrm{V}_{\max x}, \cdot s^{-1}$ & $2860 \pm 830$ & $2580 \pm 700$ \\
\hline
\end{tabular}

Isometric properties of dorsal flexors ( $\mathrm{n}=10 \mathrm{in}$ each group) were assessed at $125 \mathrm{~Hz}, 0.5 \mathrm{~ms}$, and supra-maximal stimulation current. Duration of the tetanic contraction amounted to $150 \mathrm{~ms}$. Shortening properties were assessed using the stimulation parameters as indicated in table 8.1. $\mathrm{V}_{\mathrm{op}}$ accounts for the optimal angular velocity, and $\mathrm{V}_{\max }$ is the maximal angular velocity. Statistical differences between wild type and GLUT4 ${ }^{-1}$ are indicated by * $(\mathrm{p}<0.05)$. 


\subsubsection{Fatigue protocol}

During the course of the series of 150 contractions, the torque output for each individual contraction showed a substantial decline for both wild type and GLUT4 ${ }^{-1}$ dorsal flexors as is indicated in figure 8.1. This figure shows a representative set of torque tracings registered at the $1^{\text {st }}(\mathrm{a}), 30^{\text {th }}(\mathrm{b})$, and $150^{\text {th }}(\mathrm{c})$ contraction.

figure 8.1 Representative torque tracings at different stages of the 150 shortening contraction series
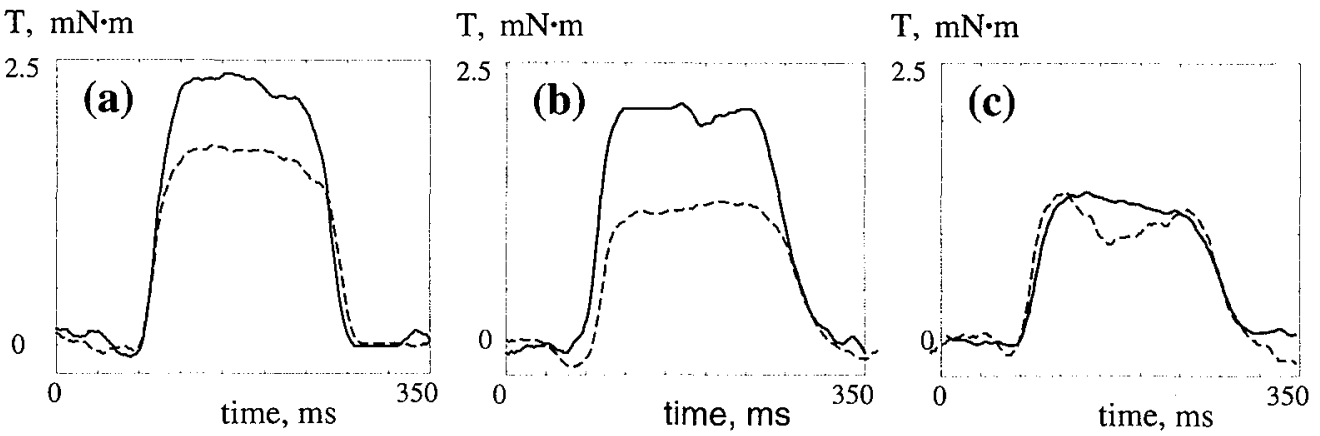

$a, b$, and $c$ correspond with the $1^{\text {st }}, 30^{\text {th }}$, and $150^{\text {th }}$ contraction, respectively. Solid and dashed lines refer to wild type and GLUT4 ${ }^{-/}$mice, respectively. The dorsal flexors were stimulated with $125 \mathrm{~Hz}$ for $200 \mathrm{~ms}$, at $1 \mathrm{~Hz}$ angular frequency with a $20^{\circ}$ stroke.

To explore in more detail possible differences in fatigability between GLUT4 $^{-1-}$ and wild type muscles, the work output of each individual contraction was calculated. The results are shown in figure 8.2a. The pattern of the changes in work output underscores the notion that the dorsal flexors of both GLUT4 ${ }^{-1}$ and wild type mice are subject to fatigue during the contraction protocol. Figure $8.2 \mathrm{~b}$ shows work output during the course of the protocol as percentage of the value obtained at the first contraction. Statistical analysis clearly revealed that the decline in work is more outspoken in the GLUT4 $^{-1-}$ mice between the $12^{\text {th }}$ and $60^{\text {th }}$ contraction $(\mathrm{p}<0.05)$ when compared to corresponding contractions in the wild type group.

Steady state work output was defined as the mean work output over the last 40 contractions. Both absolute and relative steady state work outputs per unit mass were significantly declined in GLUT4 ${ }^{-/-}$when compared to wild type. Relative steady state work output amounted to $42.7 \pm 6.5 \%$ and $35.4 \pm 0.4 \%$ of the work output of the first contraction for wild type dorsal flexors and GLUT4 ${ }^{-1-}$ dorsal flexors, respectively. 
figure 8.2 Dorsal flexor work output normalised on muscle complex mass (a) and \% of initial work output (b) during 150 shortening contractions in wild type $(\diamond)$ and GLUT4 ${ }^{-/-}(\square)$ mice.
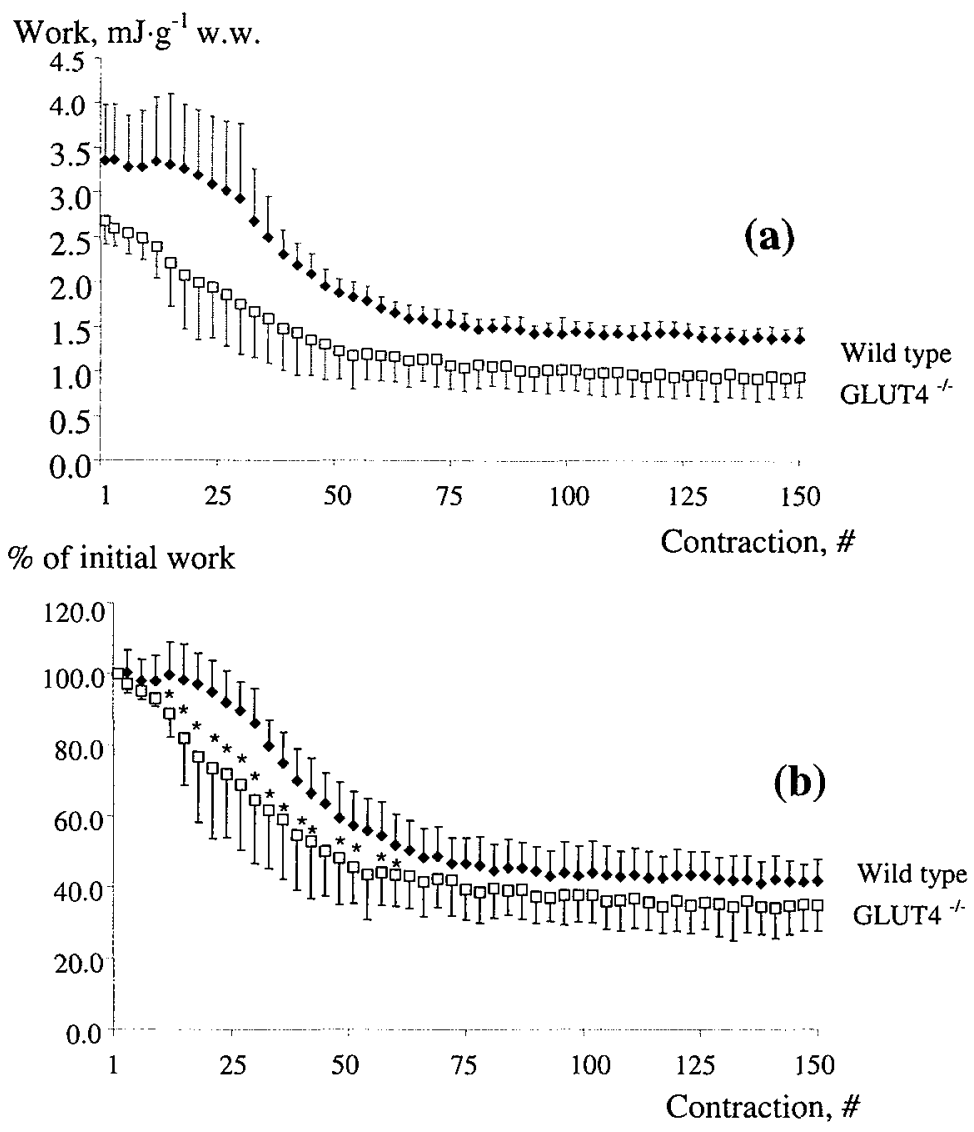

Total duration of the contraction protocol amounted to $300 \mathrm{~s}$. The murine muscles were electrically stimulated at $125 \mathrm{~Hz}$ for $200 \mathrm{~ms}$ every $2000 \mathrm{~ms}$. Symbols and vertical lines shown in figure refer to mean and SD, respectively ( $\mathrm{n}=10$ in each group). For figure $8.2 \mathrm{a}$, all data points of GLUT4 $4^{-f-}$ mice were statistically lower than the wild type littermates $(\mathrm{p}<0.05)$. Statistical differences between wild type and GLUT4 ${ }^{-/-}$in figure $8.2 \mathrm{~b}$ are indicated by $*(\mathrm{p}<0.05)$.

\subsubsection{Glycogen and high-energy phosphate content}

Resting muscular glycogen content was significantly lower in GLUT4 ${ }^{-/-}$dorsal flexors than in wild type dorsal muscles (table 8.4). Following the 150 shortening contractions, wild type mice showed a significant decline of muscle glycogen from $112 \pm 16$ to $66 \pm 12 \mu \mathrm{mol}$ glycosyl units $\cdot \mathrm{g}^{-1}$ d.w.. In contrast, GLUT4 $4^{-1}$ dorsal flexors showed no statistically significant decline in glycogen, i.e., pre- and post-exercise levels amounted to $74 \pm 19$ and $55 \pm 22 \mu \mathrm{mol}$ glycosyl units $\cdot \mathrm{g}^{-1} \mathrm{~d}$.w., respectively. 
The content of phosphocreatine and creatine in resting dorsal flexor muscle complex did not differ between wild type and GLUT4 ${ }^{-/}$mice (table 8.5 ). The same holds for tissue levels of adenine nucleotides and related compounds.

table 8.4 Glycogen content in dorsal flexors of wild type and GLUT4 ${ }^{-1-}$ mice during rest and after the fatigue protocol.

\begin{tabular}{lcc}
\hline \hline glycogen content & wild type & GLUT4 $^{-/}$ \\
& & $74 \pm 19 *$ \\
\cline { 2 - 3 } & $112 \pm 16$ & $55 \pm 22$ \\
resting muscle & $66 \pm 13 \#$ & \\
fatigued muscle & &
\end{tabular}

Data refer to mean and SD and is expressed as $\mu \mathrm{mol}$ glycosyl units $\mathrm{g}^{-1}$ dry weight ( $\mathrm{n}=6$ in each group). Statistical differences between wild type and GLUT $4^{-1}$ are indicated by $*(\mathrm{p}<0.05)$. Statistical differences in glycogen content between muscles in rest and after the fatigue protocol are indicated by $\#(p<0.05)$.

table 8.5 The content of high-energy phosphates and associated compounds in resting dorsal flexors of wild type and GLUT4 ${ }^{-/}$mice.

\begin{tabular}{lll}
\hline & wild type & \\
& & GLUT4 $^{--}$ \\
\cline { 2 - 3 } ATP & $32.9 \pm 2.4$ & \\
ADP & $4.9 \pm 0.9$ & $31.4 \pm 2.8$ \\
AMP & $0.4 \pm 0.0$ & $4.8 \pm 0.6$ \\
TAN & $38.4 \pm 2.4$ & $0.5 \pm 0.1$ \\
IMP & $0.4 \pm 0.1$ & $36.7 \pm 2.8$ \\
PCr & $90.1 \pm 9.3$ & $0.5 \pm 0.1$ \\
Cr & $76.8 \pm 17.5$ & $91.9 \pm 10.7$ \\
PCr $+\mathrm{Cr}$ & $166.9 \pm 10.8$ & $82.3 \pm 5.6$ \\
$\mathrm{PCr} / \mathrm{Cr}$ & $1.2 \pm 0.5$ & $174.2 \pm 13.3$ \\
\hline
\end{tabular}

Data refer to mean and $\mathrm{SD}$ and is expressed as $\mu \mathrm{mol} \cdot \mathrm{g}^{-1}$ dry weight ( $\mathrm{n}=6$ in each group). The ratio phosphocreatine/creatine $(\mathrm{PCr} / \mathrm{Cr}$ ) is dimensionless. Total adenine nucleotide content (TAN) was computed as the sum of ATP, ADP, and AMP. Statistical differences between wild type and GLUT $4^{\%}$ were absent.

\subsubsection{Fibre type composition and fibre cross sectional area}

Both in tibialis anterior muscle and the EDL muscle, GLUT4 ${ }^{-/}$mice showed a significant shift from type IIB to IIA fibres compared to the fibre distribution in the corresponding wild type muscles (table 8.6). The relative area of the type IIB fibres was significantly decreased in GLUT $4^{-{ }^{-}}$, whereas the relative area of IIA fibres increased. The cross-sectional area of the single fibres belonging to the respective subtypes did not differ between wild type and GLUT4 $4^{-/}$, neither in tibialis anterior muscle nor in EDL muscle. 
table 8.6 Fibre type distribution and cross-sectional area (CSA) of single and 100 counted fibres of the m. tibialis anterior and $\mathrm{m}$. EDL in wild type and GLUT4 ${ }^{-1-}$ mice.

\begin{tabular}{|c|c|c|c|c|c|c|}
\hline & \multicolumn{2}{|c|}{ fibre type distribution, $\%$} & \multicolumn{2}{|c|}{$\mathrm{CSA}, \mu m^{2}$} & \multicolumn{2}{|c|}{$\mathrm{CSA}, \%$} \\
\hline & wild type & GLUT4 ${ }^{-1-}$ & wild type & GLUT4 ${ }^{-1-}$ & wild type & GLUT4 $4^{-1-}$ \\
\hline \multicolumn{7}{|l|}{ m. tibialis anterior } \\
\hline type IIA & $15 \pm 6$ & $31 \pm 5 *$ & $650 \pm 250$ & $620 \pm 140$ & $7 \pm 3$ & $17 \pm 5 *$ \\
\hline type IIB & $83 \pm 5$ & $62 \pm 6 *$ & $1230 \pm 290$ & $1460 \pm 360$ & $90 \pm 2$ & $79 \pm 9 *$ \\
\hline miscellaneous & $2 \pm 1$ & $7 \pm 3 *$ & $740 \pm 300$ & $700 \pm 120$ & $3 \pm 2$ & $5 \pm 2$ \\
\hline \multicolumn{7}{|l|}{ m. EDL } \\
\hline type I & $8 \pm 1$ & $12 \pm 4$ & $480 \pm 140$ & $730 \pm 300$ & $3 \pm 2$ & $7 \pm 2 *$ \\
\hline type IIA & $18 \pm 4$ & $35 \pm 15^{*}$ & $650 \pm 290$ & $700 \pm 220$ & $11 \pm 7$ & $20 \pm 6$ \\
\hline type IIB & $70 \pm 5$ & $47 \pm 17 *$ & $1310 \pm 200$ & $1700 \pm 520$ & $82 \pm 10$ & $68 \pm 9 *$ \\
\hline miscellaneous & $4 \pm 3$ & $5 \pm 3$ & $1040 \pm 450$ & $1030 \pm 300$ & $4 \pm 2$ & $4 \pm 2$ \\
\hline
\end{tabular}

Data refer to mean and $\mathrm{SD}$ ( $\mathrm{n}=4$ in each group). The relative fibre type distribution is expressed as percentage of all fibres counted. Cross-sectional area is either expressed as $\mu \mathrm{m}^{2}$ (mean CSA of single fibres) or as percentage of the total area occupied by all fibres counted. Fibres are typed miscellaneous when no distinction could be made between IIA and IIB fibres. Statistical differences between wild type and GLUT4 ${ }^{-1}$ are indicated by * $(p<0.05)$.

\section{5 - Discussion}

Uptake of the bulk of glucose by skeletal muscle tissue is facilitated by GLUT1 and GLUT4, where GLUT4 is the predominant muscular glucose transporter in muscular tissue following a contraction stimulus. Here we provide more insight in the consequence of GLUT4 deficiency for muscular contractile performance of the intact dorsal flexors during a single isometric and shortening contraction. Moreover, we have explored whether GLUT4 deficiency results in enhanced fatigability of the dorsal flexor complex in its natural surrounding during repetitive shortening contractions.

\subsubsection{Single isometric tetanic contraction}

The present findings show that the maximal tetanic torque output in dorsal flexors of GLUT4 ${ }^{-1-}$ is significantly declined compared to wild type littermates. Contractile performance under isometric conditions is, among others, dependent on the cellular availibility of high-energy phosphates and on muscular mass. The content of high-energy phosphates was found comparable between wild type and GLUT4 ${ }^{- \text {- }}$ dorsal flexors (table 8.5). These findings make a decisive role of high-energy phosphates and related compounds in the decline in isometric torque in GLUT4 ${ }^{-1-}$ dorsal flexors less likely. However, the mass of the dorsal flexors of $\mathrm{GLUT}^{-{ }^{--}}$mice was significantly lower in GLUT4 ${ }^{-1-}$ mice than in wild type mice. 
When the tetanic torque was normalised per unit muscle mass, no significant difference between GLUT $4^{-/-}$and the wild type littermates was observed (table 8.3). These results corroborate with earlier findings in an isolated muscle preparation, which showed that isometric peak tension per unit muscle mass was comparable between GLUT4 $^{-1-}$ and wild type mice (13).

\subsubsection{Muscular performance during shortening contractions}

In contrast to contractile performance during isometric contractions, the maximal peak power, even after normalisation per unit muscle mass, was significantly lower in GLUT4 deficient muscle complex than in the dorsal flexors of wild type littermates ( 0.26 vs. $\left.0.36 \mathrm{~W} \cdot \mathrm{g}^{-1}\right)$. Peak power output depends, among others, on composition of muscle fibres. This notion is supported by data in literature about differences in power output of distinct types of skinned fibres (25-27). Bottinelli and co-workers reported that peak power of rat skinned fibres, measured at $12^{\circ} \mathrm{C}$, was higher for fast-twitch glycolytic fibres than fast-twitch oxidative fibres, i.e., ratio for peak power of IIB and IIA was $1.5: 1$ (27). Since in both the tibialis anterior muscle and the EDL muscle in GLUT4 ${ }^{-1}$ the relative contribution of IIA fibres to the crosssectional area increases at the expense of IIB fibres. It is tempting to state that the change in fibre type composition in GLUT4 ${ }^{-/}$dorsal flexors may be one of the causes for the lower peak power per unit muscle mass compared to wild type littermates.

\subsubsection{Fatigue protocol}

During the course of 150 shortening contractions, work output of the dorsal flexors of both wild type and GLUT4 ${ }^{-/-}$showed a substantial decline. It is known that the intracellular glycogen pool in combination with blood glucose serves as energy store during exercise of moderate to high intensity $(3,28)$. These findings are underscored by the present observation that during the contraction series the glycogen store in dorsal flexor muscle of wild type mice significantly decreased with $46 \pm 10$ $\mu$ mol glycosyl units.g ${ }^{-1}$ dry weight. Support for the assumption that blood borne glucose serves as a substrate in our contraction protocol stems from studies by Ryder and co-workers, who showed a 6 fold increase in muscular 2-deoxyglucose uptake in wild type mice submitted to a comparable contraction protocol (13). Our data indicate that work output per unit muscle mass during the whole course of the contraction series was significant decreased in GLUT4 ${ }^{-1-}$ mice. When work output was normalised for the work output at the initial contraction, the decline in work is more outspoken between the $12^{\text {th }}$ and $60^{\text {th }}$ contraction in mice deficient in GLUT4 (fig. 8.2b), which points to a higher susceptibility to fatigue in GLUT4 deficient mice. 
Many factors have been proposed to cause fatigue and strong evidence for a relation between fatigue and available glycogen is the consistent observation that fatigue during moderate exercise is associated with glycogen depletion (29-31). It is, therefore, likely that the low glycogen content in dorsal flexors deficient in GLUT4 contributes to the observed increase in fatigability.

The lower basal glycogen store in GLUT4 $4^{-/}$dorsal flexors than in wild type is in accordance with observations of other investigators, i.e., a decline in glycogen in EDL muscle in GLUT4 ${ }^{-/-}$muscle (32). The cause of the reduced glycogen content in resting dorsal flexor muscles of GLUT4 deficient mice is basically unknown. The relative increase in type IIA fibres can be dismissed as possible cause, since Azpiazu and coworkers recently showed that there was no difference in resting glycogen level between IIA and IIB of hindlimb muscles of the mouse (33). The cellular level of glycogen is governed by the rate of synthesis and degradation. Under steady state (or resting) conditions, synthesis and degradation are in equilibrium. The rate of synthesis is dependent on the availability of blood glucose, its transport across the sarcolemma and the actual activity of the enzymes involved in the incorporation of glucose into glycogen. Since Ryder and co-workers reported that blood glucose levels are similar between fed wild type and GLUT4 ${ }^{-/}$mice (13), glucose availability is not disturbed. The authors also showed that basal glucose uptake was not significantly different between wild type and GLUT4 ${ }^{-/}$EDL muscle (13). To explain this phenomenon, the authors speculated that another isoform of the muscular GLUT family compensates for the loss of GLUT4 under resting conditions. These considerations suggest that the intracellular capacity to synthesise glycogen is altered in the GLUT4 deficient muscles. The underlying mechanisms remain, however, to be elucidated.

Relative steady state work was significantly lower in GLUT4 ${ }^{-1-}$ than in wild type dorsal flexors. This finding strongly suggest that the energy supply in the steady state is lower in $\mathrm{GLUT}^{-/}$mice than in wild type littermates. A possible energy source during the steady state phase is blood borne glucose. Ryder and co-workers recently showed a four times lower 2-deoxyglucose uptake in contracting GLUT $^{-/-}$muscles than in wild type muscles (13). The apparent insufficient increase in uptake of blood borne glucose might be compensated by enhanced utilisation of other oxidative substances such as fatty acids. The significant lower output in relative steady state work, however, indicates that energy supply through alternative routes is not capable to fully compensate for the lack of GLUT4. 


\section{6 - Acknowledgement}

We gratefully acknowledge Dr. H. van Straaten from the Department of Anatomy/Embryology, Maastricht University, The Netherlands for his support during the analysis of the muscle fibre composition. 


\section{7 - References}

1. Burant, C. F., Sivitz, W. I., Fukumoto, H., Kayano, T., Nagamatsu, S., Seino, S., Pessin, J. E., and Bell, G. I. (1991) Mammalian glucose transporters: structure and molecular regulation. Rec. Prog. Horm. Res. 47, 349-388

2. McComas, A. J. (1996) Skeletal muscle, form and function, Human Kinetics, Leeds

3. Hargreaves, M. (1997) Interactions between muscle glycogen and blood glucose during exercise. Ex. Sport Sci. Rev. 25, 21-38

4. Romijn, J. A., Coyle, E. F., Sidossis, S., Gastaldelli, A., Horowitz, J. F., Endert, E., and Wolfe, R. R. (1993) Regulation of endogenous fat and carbohydrate metabolism in relation to exercise intensity and duration. Am. J. Physiol. 265, E380-E391

5. Bell, G. I., Kayano, T. K., Buse, J. B., Burant, C. F., Takeda, J., Lin, D., Fukumoto, H., and Seino, S. (1990) Molecular biology of mammalian glucose transporters. Diab. Care 13, 198-208

6. Brozinick, J. T., Yaspelkis, B. R., Wilson, C. M., and Grant, K. E. (1996) Glucose transport and GLUT4 protein distribution in skeletal muscle. Science 313, 133-140

7. Klip, A., Volchuk, A., He, L., and Tsakidiris, T. (1996) The glucose transporter of skeletal muscle. Cell Dev. Biol. 7, 229-237

8. Rodnick, K. J., Slot, J. W., Studelska, D. R., Hanpeter, D. E., Robinson, L. J., Geuze, H. J., and James, D. E. (1992) Immunocytochemical and biochemical studies of Glut4 in rat skeletal muscle. J. Biol. Chem. 267, 6278-6285

9. Slot, J. W., Geuze, H. J., Gigengack, S., Lienhard, G. E., and James, D. E. (1991) Immuno-localization of the insulin regutable glucose transporter in brown adipose tissue in rat. J. Cell Biol. 133, 123-135

10. Slot, J. W., Geuze, H. J., Gigengack, S., James, D. E., and Lienhard, G. E. (1991) Translocation of the glucose transporter GLUT4 in cardiac myocytes of the rat. Proc. Natl. Acad. Sci. 88, 7815-7819

11. Bergstrom, J., and Hultman, E. (1966) Muscle glycogen synthesis after exercise: an enhancing factor localized to the muscle cells in man. Nature 210, 309-310

12. Richter, E. A., and Galbo, H. (1986) High glycogen levels enhance glycogen breakdown in isolated contracting skeletal muscle. J. Appl. Physiol. 61, 827-831

13. Ryder, J. W., Kawano, Y., Galuska, D., Fahlman, R., Wallberg-Henriksson, H., Charron, M. J., and Zierath, J. R. (1999) Postexercise glucose uptake and glycogen synthesis in skeletal muscle from GLUT4-deficient mice. FASEB J. 13, 2246-2256

14. Katz, E. B., Stenbit, A. E., Hatton, K., DePinho, R., and Charron, M. J. (1995) Cardiac and adipose tissue abnormalities but not diabetes in mice deficient in GLUT4. Nature $377,151-155$

15. Gorselink, M., Drost, M. R., Louw de, J., Willems, P. J. B., Rosielle, N., Janssen, J. D., and Van der Vusse, G. J. (2000) Accurate assessment of in situ isometric contractile properties of hind limb plantar and dorsal flexor muscle complex of intact mice. Pflügers Arch. 439, 665-670

16. Gorselink, M., Drost, M. R., Louw de, J., Willems, P. J. B., Dekkers, E. C. A., Janssen, J. D., and Vusse van der, G. J. (2000) In situ assessment of shortening and lengthening contraction properties of hind limb ankle flexors of intact mice. Pflügers Arch. in press

17. Hespel, P., and Richter, E. A. (1990) Glucose uptake and transport in contracting, perfused rat muscle with different pre-contraction glycogen concentrations. J. Physiol. (London) 427, 347-359

18. Edwards, R. H. T., Hill, D. K., and Jones, D. A. (1975) Metabolic changes associated with the slowing of relaxation in fatigued mouse muscle. J. Physiol. 251, 287-301 
19. Ruiter, C. J., Jones, D. A., Sargeant, A. J., and Haan, A. D. (1999) Temperature effect on the rates of isometric force development and relaxation in the fresh and fatigued human adductor pollicis muscle. Pflügers Arch. 84, 1137-1150

20. Hill, A. V. (1938) The heat of shortening and the dynamic constants of muscle. Proc. Roy. Soc. London B. 126, 136-195

21. Van der Vusse, G. J., Coumans, W. A., Van der Veen, F. H., Drake, A., Flameng, W., and Suy, R. (1984) ATP, creatine phosphate and glycogen content in human myocardial biopsies: markers for the efficacy of cardioprotection. Vasc. Surg. 8, 127134

22. Wijnants, J., and van Belle, H. (1985) Single-run high performance chromatography of nucleotides, nucleosides and major purine bases and its application to different tissue extracts. Anal. Biochem. 144, 258-266

23. Lowry, O. H., and Pasonneau, J. V. (1972) A flexible system of enzymatic analysis, Academic Press, New York

24. Brooke, M. H., and Kaiser, K. K. (1970) Muscle fibre types: how many and what kind? Arch. Neurol. 23, 369-379

25. Widrick, J. J., Trappe, S. W., Costill, D. L., and Fitts, R. H. (1996) Force-velocity and force-power properties of single muscle fibres from elite master runners and sedentary men. Am. J. Physiol. 271, C676-683

26. McDonald, K. S., Blaser, C. A., and Fitts, R. H. (1994) Force-velocity and power characteristics of rat soleus muscle fibres after hindlimb suspension. J. Appl. Physiol. $77,1609-1616$

27. Bottinelli, R., Schiaffino, S., and Reggiani, C. (1991) Force-velocity relations and myosin heavy chain isoform compositions of skinned fibres from rat skeletal muscle. J. Physiol. (London) 437, 655-672

28. Bonen, A., McDermott, J. C., and Hutber, C. A. (1989) Carbohydrate metabolism in skeletal muscle: an update of current concepts. Int. J. Sports Med. 10, 385-401

29. Allen, D. G., Lannergren, J., and Westerblad, H. (1995) Muscle cell function during prolonged activity: cellular mechanisms of fatigue. Exp. Physiol. 80, 497-527

30. Bergstrom, J., Hermansen, L., Hultman, E., and Saltin, B. (1967) Diet, muscle glycogen and physical performance. Acta Physiol. Scand. 71, 140-150

31. Bergstrom, J., and Hultman, E. (1967) A study of the glycogen metabolism during exercise in man. Scand. J. Clin. Lab. Invest. 19, 218-228

32. Stenbit, A. E., Burcelin, R., Katz, E. B., Tsao, T. S., Gautier, N., Charron, M. J., and Le Marchand-Brustel, Y. (1996) Diverse effects of Glut 4 ablation on glucose uptake and glycogen synthesis in red and white skeletal muscle. J. Clin. Invest. 98, 629-634

33. Azpiazu, I., Manchester, J., Skurat, A. V., Roach, P. J., and Lawrence, J. C. (2000) Control of glycogen synthesis is shared between glucose transport and glycogen synthase in skeletal muscle fibres. Am. J. Physiol. 278, E234-243 
Chapter 8

$138 \mid$ 


\section{Chapter 9}

\section{ENERAL DISCUSSION}

General discussion of the major findings in the preceding chapters, with special reference to the assessment of muscular performance in the intact mouse and energy metabolism in the contracting muscle. 


\section{1 - Study objectives and experimental approach}

The first objective of the present thesis was to develop an experimental model to determine the mechanical performance of the intact dorsal and plantar flexors of the mouse. This objective was achieved through a close co-operation between the University Maastricht (Departments of Physiology and Movement Sciences) and the Technical University Eindhoven (Departments of Biomedical Engineering and Precision Engineering). The experimental model should allow for accurate assessment of both isometric and shortening/lengthening contractile properties of the murine skeletal muscle. The second objective was to study the consequence of specific defects in energy metabolism on mechanical functioning of murine skeletal muscle. First, we studied the effect of disruption of the cytoplasmic and mitochondrial isoforms of the creatine kinase gene on isometric muscular performance in situ. Second, we investigated whether the lack of the glucose transporter (GLUT4) affects isometric and shortening contractile performance of the dorsal flexor muscle complex in situ.

\section{2 - Assessment of mechanical functioning of the intact murine muscle complex}

Chapter 3 gives a description of the model for in situ measurements of isometric function of the plantar or dorsal flexors of intact mouse hindlimb (1). With the isometric dynamometer, muscular torque of the intact muscle complexes could be accurately measured within the range of $-14 \mathrm{mN} \cdot \mathrm{m}$ and $+14 \mathrm{mN} \cdot \mathrm{m}$. Measurements of ankle and knee displacement, due to non-rigid fixation of the lower hind limb revealed that muscle fibres shorten by $2.5 \%$. Mathematical simulations of contractions either including or without this extra shortening, showed that this additional shortening had only minor effect on calculated maximal isometric torque and the temporal parameters. Furthermore, we observed that isometric torque in our set-up is hardly affected by a misalignment up to $1.0 \mathrm{~mm}$ (eccentricity) between the ankle joint and measurement axis. Although the isometric dynamometer provides valuable information on isometric features of muscle contractions of the mouse, it is not appropriate for determining the mechanical properties of shortening and lengthening contractions. Chapter 4 provides a detailed description of the mouse ergometer for assessment of shortening and lengthening contractile properties of intact plantar and dorsal flexors (2). Evaluation of the fixation procedure via $3-\mathrm{D}$ monitoring of the muscle-tendon complex length showed that the additional shortening in length, due to loose fixation during supramaximal stimulation, exerts only minor effects on torque developed by the flexor muscles. Furthermore, it was found that only an unrealistically high misalignment between the rotation axis of the ankle and measurement axis of more than approximately $2.0 \mathrm{~mm}$ substantially affected the maximal torque output. During routine experiments, however, eccentricity will never exceed $1.0 \mathrm{~mm}$. 
So, the small and inevitable eccentricity between the ankle joint and measurement axis will not affect the contractile properties determined in our experimental set-up. The experimental model developed in the present study is, therefore, a suitable tool to noninvasively assess mechanical characteristics of isometric as well as shortening and lengthening contractions of the intact hind limb ankle flexors of the mouse.

Muscle contractile properties in mice are mostly studied under in vitro experimental conditions. The references included in this chapter serve as a brief summary of studies performed under the in vitro settings (3-10). The set-ups commonly used in in vitro studies ranged from single muscle fibres to whole muscle complexes. In vitro isometric muscle function is routinely assessed by dissecting the fibres or muscle from the surrounding muscular tissue, and attach the distal tendon of the muscle or the distal part of the fibre to a force transducer. Via electrical stimulation of the muscle or fibre, isometric force development can be monitored. The same holds for the in vitro assessment of shortening and lengthening properties, but under these conditions the other end of the muscle or fibre is not connected to the fixed world but to a servomotor, with which the length of the muscle or fibre can be shortened or lengthened during electrical stimulation. A potential drawback of this invasive approach is that the surgical procedure of dissecting the muscle or fibres of interest often results in damage of the tissue, i.e., injury of the blood vessels, nerves, or the muscle fibre itself. During the measurement, the mechanical function of the muscle can be negatively affected by for example hypoxic conditions. Moreover, temperature during the experiments must be kept below $25^{\circ} \mathrm{C}$, to prevent rapid deterioration of the muscle or fibre during the course of the experiment. The low temperature itself will affect the performance of the muscle or fibre studied compared to the physiological situation. This invasive approach to investigate skeletal muscle functioning in the mouse may lead to misinterpretations, when extrapolating the results towards the in vivo situation. Furthermore, these in vitro models also restrict the investigator to acute experiments, and analysis of the long-term effects of a specific intervention using the animal as its own control is not possible. On the other hand, these in vitro models allow for discrimination between specific muscle or fibre types under standardised conditions. In the in situ set-up presented here, the above mentioned problems of invasive surgical procedure and acute experiments are overcome via the non-invasive fixation procedure leaving the muscle complex in its natural surrounding. With our experimental model, however, force developed by the muscle of interest cannot be directly determined. Force can be calculated from the torque data, if the length of the lever arm of the muscle complex is known since torque equals the product of force and the length of the lever-arm. 
Pioneering studies concerning the assessment of the mechanical activity of intact muscle complexes were performed by Ashton-Miller and co-workers (11). Their experimental set-up enables the investigators to assess muscular torque around the axis of the measurement device with a pair of small strain gauges. On theoretical grounds, it was inferred that assessment of muscle torque either with strain gauges or with a torque transducer as used in our set-up does not result in large differences in the signal-to-noise ratio. Muscle torque assessment with strain gauges, however, results in a more compliant measurement set-up. The lower compliance of our experimental model most likely provides a more accurate determination of the temporal parameters of the isometric contraction compared to the Ashton-Miller set-up. Moreover, the Ashton-Millers set-up uses a rigid knee fixation system. A rigid knee fixation may introduce additional non-muscular torques, especially when eccentricity between the axis of rotation of the ankle joint and the measurement axis exists, which introduces a crankshaft at the region of the ankle. Consequently, during angular oscillation, the crankshaft mechanism will introduce movements at the level of the knee. As a consequence, during a rigid knee fixation non-contractile forces will be transmitted via the tibia to the measurement axis. This extra force in combination with the eccentricity will introduce a confounding non-muscular torque (chapter 3, figure 3.1b). We have prevented this problem by designing a novel hip-fixation unit, and data presented in chapter 3 and 4 show that inevitable eccentricity does not substantially affect torque during isometric or shortening contractions during our experiments.

Table 9.1 gives a brief overview of the in situ contractile parameters assessed in chapter 3 to 8 for the intact dorsal and plantar flexors of the Swiss and C57/bl6 mouse. These findings show that the values of the contractile parameters depend, among others, on the type of the muscle complex studied. Maximal isometric torque was significantly lower in dorsal than in plantar flexors. This can partly be explained by the difference in muscle mass, but torque per unit muscle mass was still significantly lower in the dorsal flexors. Data in literature showed that the lever arm of the plantar and the dorsal flexor complex amounted to approximately $2.1 \mathrm{~mm}$ and $1.0 \mathrm{~mm}$, respectively. Using these values, force per unit muscle mass was calculated to be in the range of 35.8 to $36.9 \mathrm{~N} \cdot \mathrm{g}^{-1}$ and 33.5 to $35.4 \mathrm{~N} \cdot \mathrm{g}^{-1}$ for the plantar and dorsal flexors, respectively. So, in both muscle complexes an approximately equal amount of isometric force per unit mass can be generated. 
table 9.1 Contractile properties of the intact dorsal and plantar flexors of the Swiss and C57bl/6 mice.

\begin{tabular}{|c|c|c|c|c|}
\hline & \multicolumn{2}{|c|}{ Swiss (16 wk) } & \multicolumn{2}{|c|}{ C57/bl6 (8-12 wk) } \\
\hline & plantar flexors & dorsal flexors & plantar flexors & dorsal flexors \\
\hline \multicolumn{5}{|l|}{ Isometric conditions } \\
\hline torque, $m N \cdot m$ & $11.8 \pm 1.6$ & $3.2 \pm 0.1 *$ & $9.4 \pm 1.4$ & $2.2 \pm 0.4 *$ \\
\hline torque per unit muscle mass, $m N \cdot m \cdot g^{-l}$ & $77.4 \pm 10.5$ & $35.4 \pm 1.7 *$ & $75.2 \pm 13.0$ & $33.5 \pm 3.6 *$ \\
\hline $10-50 \%$ rise time, $m s$ & $14.9 \pm 0.6$ & $11.8 \pm 2.0 *$ & $15.9 \pm 2.1$ & $11.2 \pm 1.3 *$ \\
\hline $1 / 2$-relaxation time, $m s$ & $13.4 \pm 1.2$ & $10.4 \pm 1.1 *$ & $13.5 \pm 3.9$ & $11.5 \pm 2.8$ \\
\hline Shortening conditions & & & & \\
\hline peak power per unit muscle mass. $W \cdot g^{-l}$ & $0.19 \pm 0.03$ & $0.27 \pm 0.02 *$ & n.m. & $0.36 \pm 0.09$ \\
\hline optimal shortening velocity, ${ }^{n} \cdot s^{-1}$ & $700 \pm 90$ & $1100 \pm 190 *$ & n.m. & $1330 \pm 300$ \\
\hline maximal shortening velocity, ${ }^{\prime \prime} \cdot s^{-1}$ & $2580 \pm 180$ & $3420 \pm 380 *$ & n.m. & $2860 \pm 830$ \\
\hline
\end{tabular}

Data are expressed as mean \pm SD ( $n=8$ for the Swiss mice and $n=20$ for the $C 57 \mathrm{~b} / 6$ ). Significant differences between the plantar and dorsal flexors are indicated by $*(p<0.05)$, and were analysed using the non-parametric Mann-Whitney U test. Shortening contractions in the plantar flexors were not assessed (n.m.).

The maximal peak power, even after normalisation per unit muscle mass, was significantly lower in the plantar flexor than in the dorsal flexor muscle complex (table 9.1). Peak power output depends, among others, on the relative distribution of muscle fibre types. Peak power of rat skinned fibres, measured at $12^{\circ} \mathrm{C}$, was approximately 1.5 times higher for fast-twitch glycolytic (IIB) fibres than for fast-twitch oxidative (IIA) fibres (12). Since the relative volume of IIA fibres in the plantar flexors is larger than in the dorsal flexors (13), it is tempting to state that the difference in muscle fibre type composition is the main cause for the lower peak power per unit muscle mass in the plantar flexors.

\section{3 - Role of creatine kinase in muscle mechanical performance}

Creatine kinase $(\mathrm{CK})$ is abundantly present in excitable tissues, including muscle and brain (14). CK catalyses the exchange of the high-energy phosphate group between phosphocreatine (PCr) and ATP (eqn. 9.1):

$$
\mathrm{MgADP}^{-}+\mathrm{PCr}^{2-}+\mathrm{H}^{+} \leftrightarrow \mathrm{MgATP}^{2-}+\mathrm{Cr}
$$

Until know, five different $\mathrm{CK}$ iso-enzymes have been identified in mammalian tissues. The adult skeletal muscle contains the cytosolic MM-CK and the mitochondrial $\mathrm{M}_{\mathrm{i}}$ CK isoforms $(14,15)$. The bulk of $\mathrm{M}_{\mathrm{i}}-\mathrm{CK}$ is bound to the outer side of the inner membrane of the mitochondria, whereas MM-CK is both freely dispersed in the cytosol and associated with intracellular structures, such as the myofibrillar M-band, glycolytic enzyme complexes and the membrane of the sarcoplasmatic reticulum (SR) (16). 
Based on the CK-isoenzyme diversity and the subcellular distribution, it has been proposed that the CK system has distinct functions (see for review in (14)), including the temporal and spatial buffering of the cellular ATP concentration (see below).

Wieringa and co-workers have developed several mouse models deficient in one or more of the muscular $\mathrm{CK}$ isoenzymes that offer unique possibilities for creatine kinase research, i.e., $\mathrm{MM}-\mathrm{CK}^{-/}(17), \mathrm{M}_{\mathrm{i}}-\mathrm{CK}^{-1}$ (18), and $\mathrm{MM} \times \mathrm{M}_{\mathrm{i}}-\mathrm{CK}^{-1}$ (19). This thesis (chapter 5 to 7) gives an overview of consequences of ablation of both MM-CK and $\mathrm{M}_{\mathrm{i}} \mathrm{-CK}$ on muscular performance and high-energy phosphate handling in the mouse (19). The significantly lower torque and prolonged relaxation time during a single isometric contraction of both the dorsal and plantar flexors most likely indicate that supply of high-energy phosphates to both the contractile machinery and SR $\mathrm{Ca}^{2+}$ pumps is substantially impaired. These findings suggest that $\mathrm{PCr}$ is an important energy carrier for strenuous contractile activity as well as proper $\mathrm{Ca}^{2+}$ homeostasis in skeletal muscle. Moreover, the results in chapters 5 and 6 show that the torque output during a series of 12 consecutive contractions of the dorsal flexor complex of $\mathrm{CK}^{-1}$ mice is significantly depressed (20). Lower tissue ATP and higher IMP levels may contribute to this more pronounced decline in torque during a high-intensity contraction series. In contrast to wild type muscle, ATP content decreased during a series of 12 isometric tetanic contractions in dorsal flexor muscle of $\mathrm{CK}^{-1-}$ mice (chapter 5). The findings in chapter 6, however, clearly indicate that in muscles lacking both creatine kinase isoforms maximal torque developed during the first tetanic contraction of a second series of high-intensity contractions is comparable with that of the first series from which it is divided by a recovery period of $60 \mathrm{~s}$. The fact that the tissue content of ATP is appreciably lower and that of IMP significantly higher at the onset of the second contraction series than at the start of the first series makes a prominent role of these two substances in the decline in torque in $\mathrm{CK}^{-/}$muscles, when compared to wild type, less likely.

The higher tissue IMP indicates that adenylate kinase-mediated regeneration of ATP compensates for the lack of $\mathrm{CK}^{-/}$activity. However, this pathway inevitably results in a decline in tissue content of adenine nucleotides. Figures 9.1a and 9.1b provide schematic representations of the metabolic consequences of the lack of both muscular CK isoforms on high-energy phosphate handling during a contraction series of low/moderate intensity and high-intensity, respectively. 
figure 9.1a Schematic representation of the phosphate flux during a contraction series of low/moderate intensity in wild type $(\mathrm{A})$ and $\mathrm{CK}^{-/-}(\mathrm{B})$ skeletal muscle.

\section{A: wild type}

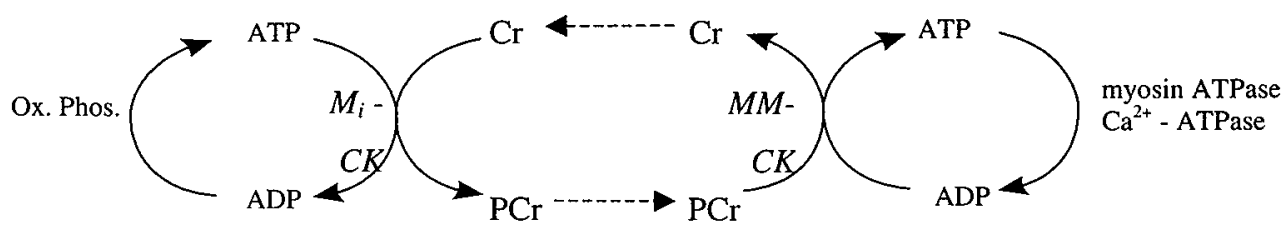

AMP/ADP shuttle

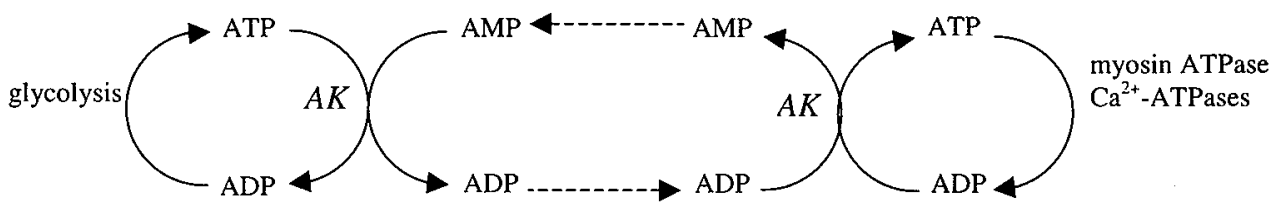

$\underline{\mathrm{B}: \mathrm{CK}^{-1-}}$
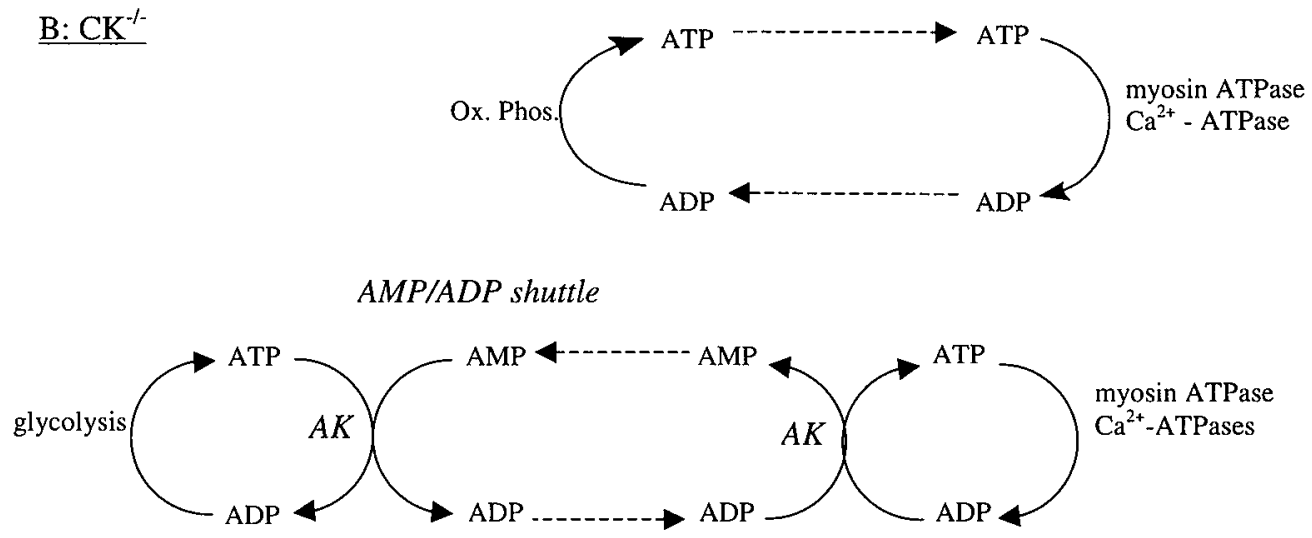

$\mathrm{M}_{\mathrm{i}}-\mathrm{CK}, \mathrm{MM}-\mathrm{CK}$, and $\mathrm{AK}$ refer to the mitochondrial and cytosolic isoforms of creatine kinase and adenylate kinase, respectively. Ox Phos. refers to the oxidative phosphorylation, $\mathrm{PCr}$ and $\mathrm{Cr}$ to phosphocreatine and creatine, respectively, and IMP to inosine monophosphate. Solid arrows indicate metabolic conversion and broken arrows indicate diffusion of the chemical substance.

Figure $9.1 \mathrm{a}$ is based on the assumption that during low/moderate contraction intensity ATP utilisation and production are completely matched. In wild type muscles, ATP utilised for contraction is partly replenished by the $\mathrm{CK}$ system, i.e., temporal buffering via the activity of MM-CK isoform. 
Since in muscle cells mitochondria are commonly not located in close vicinity of the energy consuming systems, e.g., contractile machinery and $\mathrm{Ca}^{2+}$-ATPase, the $\mathrm{CK}$ isoenzymes also serve in a transport system for the end-standing high-energy phosphate group of ATP from the mitochondria towards the site of ATP utilisation, i.e., spatial buffer system via the PCr shuttle. As has been outlined by Zeleznikar and co-workers (22), adenylate kinase plays a role in the functional coupling of the glycolytically produced ATP and ATP utilisation processes. ATP produced via the glycolytic pathway reacts in the cytoplasm with AMP yielding two molecules of ADP. This reaction step is catalysed by adenylate kinase associated with the glycolytic complex. One molecule ADP diffuses to the contractile machinery, i.e., the site of ATP consumption, and reacts with a second molecule ADP, which is previously produced during muscular contraction. This reaction, catalysed by a second set of adenylate kinase enzymes, yields ATP and AMP. AMP diffuses back to the site of glycolytic activity, while ATP is hydrolysed by myosin ATPases or sarcoplasmic reticular $\mathrm{Ca}^{2+}$ ATPases to ADP.

In CK deficient muscle cells the loss of spatial buffering by of the CK system is partly compensated by a decline in the distance between the mitochondria and the ATP utilising systems (18). Furthermore, temporal buffering of the ATP content is, among others, compensated via the adenylate kinase pathway.

figure 9.1b Schematic representation of the phosphate flux during a contraction series of high- intensity in wild type $(\mathrm{A})$ and $\mathrm{CK}^{-/}(\mathrm{B})$ skeletal muscle.
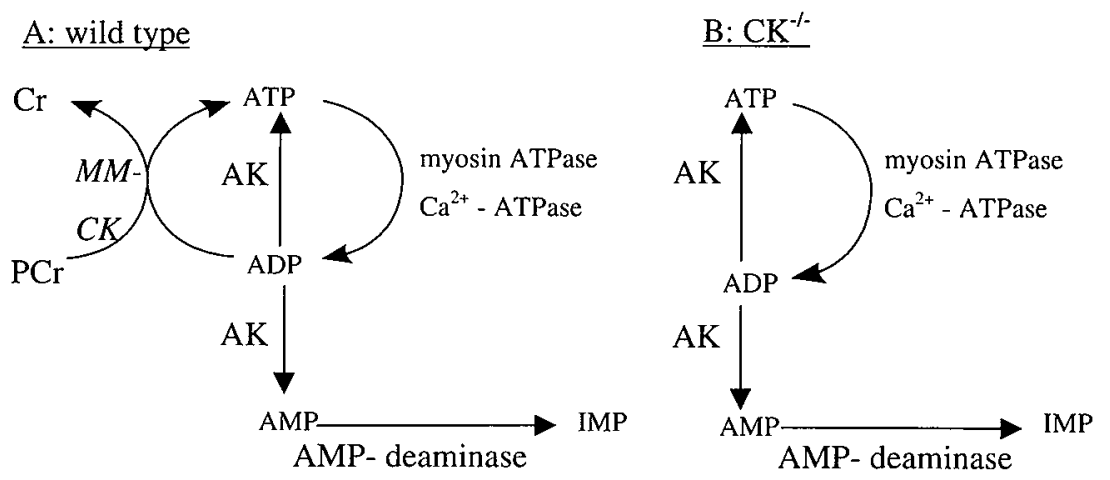

MM-CK, and AK refer to cytosolic isoforms of creatine kinase and adenylate kinase, respectively. Solid arrows indicate metabolic conversion and broken arrows indicate diffusion of the chemical substance.

In figure $9.1 \mathrm{~b}$, it is assumed that the intensity of the contraction is that high that ATP produced in the glycolytic pathway and oxidative phosphorylation is negligible. 
During high-intensity contractions of wild type muscles, ATP is temporally buffered by the PCr pool. In addition, adenylate kinase also catalysis the reaction between two molecules ADP yielding ATP and AMP. ATP is subsequently hydrolysed by myosin ATPases or sarcoplasmic reticular $\mathrm{Ca}^{2+}$ ATPases to ADP, AMP is further degraded to IMP by the catalytic action of AMP deaminase. Since creatine kinase deficient muscles are not able to rely on the temporal buffer function of creatine kinase, the adenylate kinase pathway is adequately serving in the ATP production. This pathway is, however, inherently not capable to fully compensate for the lack of $\mathrm{CK}$, since for each ATP molecule regenerated, one molecule of AMP is produced and further degraded to IMP and purine bases. ATP regeneration via the adenylate kinase pathway is, therefore, a likely cause of the relatively low basal adenine nucleotide levels in $\mathrm{CK}^{-1-}$ mice.

In conclusion, the CK system is an important energy carrier system for both contractile activity and proper $\mathrm{Ca}^{2+}$ homeostasis in skeletal muscle. The double $\mathrm{CK}$ knockout mouse has compensated this deficiency via distinct mechanisms, and these compensatory mechanisms allows the animal to fulfil all daily tasks of the mouse when housed in a normal animal cage. However, our findings clearly showed that during a severe energy challenge the $\mathrm{CK}$ knockout muscles, either during a single tetanic contraction or during a series of 12 consecutive contractions, are not able to perform as good as those of wild type littermates. Moreover, the present findings also indicate significant differences in response to $\mathrm{CK}$ ablation between dorsal and plantar flexors. Further experiments, however, are needed to fully elucidate the mechanisms underlying the muscle group-specific alterations in mechanical and cellular properties.

\section{4 - Role of GLUT4 in muscular mechanical performance.}

Glucose homeostasis is impaired in patients with non-insulin dependent diabetes, and this defect is due in part to defects of the glucose transport across the skeletal muscle cell membrane. Impaired glucose transport leads to reduced whole body glucose uptake and hyperglycaemia. Knowledge of the regulation of glucose transport in skeletal muscle is necessary to develop effective strategies to prevent the onset or reduce the consequences of non-insulin dependent diabetes. To assess the role of GLUT4 in glucose homeostasis, Katz and co-workers have generated mice, which have disrupted copies of the GLUT4 gene (23). Surprisingly, GLUT4 knockout mice have normal blood glucose levels in the fed state and show normal glucose tolerance. Therefore, the homozygous GLUT4 knockout mouse is not the appropriate model mimicking the non-insulin dependent diabetic state. On the other hand, it is a useful model to increase our knowledge about the functional role of the glucose transporter number 4 in skeletal muscle. 
Although, compensatory mechanisms for the lack of GLUT4, e.g. higher GLUT2 and GLUT1 expression in the liver and heart (23) have been reported. Nevertheless, no upregulation of any known facilitative glucose transporter in skeletal muscle in GLUT4 deficient mice has been observed. Despite the importance of regulatable glucose transporters for muscular energy metabolism, information on the impact of GLUT4 deficiency on muscle contractile performance is scarce. Our data are therefore of importance considering the physiological role of GLUT4 in contractile performance of the skeletal muscle complex in situ. The present study is the first to report the effect of GLUT4 deficiency on mechanical functioning of skeletal muscle in the intact mouse. Isometric performance (torque normalised per unit muscle mass) during a single tetanic contraction did not differ between wild type and GLUT4 deficient mice. Shortening contractions, however, revealed a significant 1.4 fold decrease in peak power per unit mass, most likely caused by fibre type transition, i.e., from fastglycolytic fibres (IIB) to fast-oxidative fibres (IIA), in GLUT4 deficient dorsal flexors. Moreover, we clearly showed that fatigability of the dorsal flexor complex subjected to a contraction protocol of moderate intensity was enhanced in GLUT4 deficient mice. The significantly lower relative work output during the steady state phase in these animals indicates that energy supply via alternative routes is not capable to fully compensate for the decline of glucose availability due to the absence of functional GLUT4. In conclusion, despite the lack of an overt phenotype of GLUT4 deficient mice, our highly sensitive experimental device enabled us to delineate significant differences in muscle contractile performance between GLUT $^{-/-}$and wild type mice under conditions of substantially enhanced energy demand of the muscle fibres.

\section{5 - Future perspectives}

Our newly developed experimental model allows for assessment of the mechanical performance during isometric, lengthening or shortening contractions of intact skeletal muscle complexes of the mouse. In order to determine tension, the golden standard in isometric muscle mechanics, methods should be implemented into the experimental procedure allowing for measurements of the length of the lever-arm and the physiological cross-sectional area (pCSA) of muscles in each experimental group of mice. Since torque equals the product of force times the length of the lever arm, knowledge of the length of the lever-arm offers the possibility to estimate the force produced by the muscle complex. Moreover, it is know that the maximal isometric force of a given muscle complex is primarily a function of the physiological cross-sectional area. Normalising the force for the cross-sectional area provides the value of developed tension $\left(\mathrm{N} \cdot \mathrm{m}^{-2}\right)$. 
Second, a major potential advantage of this in situ model compared over the in vitro methods for assessment of skeletal muscle performance is that it allows for the analysis of the long term effects of specific interventions, e.g. diet, exercise training, gene therapy. To use the animal as its own control, the fixation of the foot and the electrical stimulation procedures must be further improved. In the present experimental procedure, the mouse foot is cast in two-component cement. This material is very appropriate, because its stiffness is very high, it is easily to handle and very light in weight. It is, however, not possible to remove the cast shoe after finishing the experiment, and, therefore, the animal has to be sacrificed. The cast material should be replaced by other material with comparable specific properties, except that the material can be removed from the mouse foot without damaging the tissue. Material like plaster or specific glues should be taken into consideration.

Furthermore, the electrical stimulation procedure of the dorsal and plantar flexors should also be improved. In the present procedure, a small incision in the skin and underlying tissue is made to expose the nerve, which innervates either the dorsal or the plantar flexors. This surgical procedure results in damage of the surrounding tissue, but more importantly, the hook-electrode used will damage the myelin sheaths of the nerve. After the intervention period, stimulation of the nerve for a second time to obtain maximal torque output is therefore not possible (24). A possible solution is to replace the current hook-electrode by a pair of small needle electrodes. During the experiment, the needles should be introduced as close as possible at both sides of the nerve without touching it. Afterwards, the needles can be removed without inflicting damage on the nerve and with minimal damage of the surrounding tissue.

In the transgenic technology, the genetic defect is introduced in the embryonic stage, leaving sufficient time for adaptations in response to the deficiency induced. The CK deficient mice demonstrated that muscular tissue is able to adapt to the $\mathrm{CK}$ deficiency, which becomes apparent, among others, via a decline of the distance between the mitochondria and the ATP utilising systems in these mice (18). The same holds for GLUT4 deficient muscular tissue, in which a swift from fast-glycolytic fibres to fast-oxidative fibres is an example of the adaptive mechanism towards GLUT4 deficiency. Therefore, one must be aware of possible adaptation processes in the transgenic mouse, which may obscure the consequence, and therefore the relevance, of the deficiency of the gene of interest. The application of transgenic animals will undoubtedly contribute to an improved understanding of the functional role of specific proteins involved in muscular energy conversion. The possibility to determine the muscular contractility with our developed experimental model in a non-invasive manner will be a powerful tool in this field of research when conditional ablation of genes is applied. 
To enlarge our knowledge about the metabolic profile and structural aspects of the contracting muscle, it is of paramount importance to combine the nuclear magnetic resonance (NMR) techniques with the present experimental model. For this purpose, a new isometric dynamometer, including the novel fixation unit, should be build from non-ferrous metals. The NMR spectroscopy technique offers the possibility to assess for example the transfer of high-energy phosphates and glycogen utilisation during periods of muscular contraction. Furthermore, the length of muscle fibre, for assessment of the cross-sectional area, and the length of lever-arm could be measured with NMR imaging in a non-invasive manner.

\section{6 - Conclusions}

The main conclusions of the experiments shown in the present thesis are summarised below.

\section{Experimental Model:}

1. The isometric dynamometer allows for accurate in situ measurements of the isometric contractile parameters of plantar and dorsal flexors of intact mouse.

2. The mouse ergometer allows for accurate in situ measurements of the shortening and lengthening contractile parameters of the intact dorsal and plantar flexor complexes of the mouse, i.e., maximal peak power, and optimal and maximal shortening velocity.

3. The fixation of the hindlimb via the hip fixation unit is appropriate, and does not affect the contractile characteristics of either isometric or shortening and lengthening contractions of the plantar and dorsal flexors of the intact mouse.

4. Realistic misalignment between the ankle axis and the measurement axis of less than $1.0 \mathrm{~mm}$ has only a minor effect on the torque assessed during isometric and shortening contractions.

\section{Creatine kinase deficiency}

5. Body, dorsal flexor and plantar flexor masses of CK deficient mice are significantly lower than that of the age-matched wild type mice.

6. Both the dorsal and plantar flexors of $\mathrm{CK}$ deficient mice show a significantly lower torque per unit muscle mass than the age-matched wild type mice.

7. The rate of relaxation of a maximal tetanic contraction was significantly lower in $\mathrm{CK}^{-1-}$ mice compared to wild type muscles.

8. The relative decline in torque was significantly higher in $\mathrm{CK}^{-1}$ dorsal flexors than in wild type dorsal flexors during 12 isometric contractions of high-intensity. 
9. Mechanical performance during a second series of 12 contractions in CK deficient mice was comparable to the first series of contractions.

10. ATP regeneration via the adenylate kinase pathway in the CK deficient mouse results in a significantly higher resting and post-contraction IMP level

11. The adenylate kinase pathway in $\mathrm{CK}$ deficient mice is not capable to fully compensate for the lack of CK.

12. The compensatory adenylate kinase pathway in CK deficient mice is a likely cause of the relatively low basal adenine nucleotide levels in these mice.

13. During 12 consecutive contractions, the torque of the $\mathrm{CK}$ deficient plantar flexors exhibited a fast and substantial decline in the second contraction, which was in contrast to the gradual decline in torque observed in the $\mathrm{CK}$ deficient dorsal flexors.

\section{GLUT4 deficiency}

14. GLUT4 deficient dorsal flexors show similar isometric function when compared to wild type mice.

15. The maximal peak power per unit muscle mass is significantly lower in the GLUT4 deficient dorsal flexor muscle complex than in wild type littermates.

16. The optimal and maximal shortening velocity is significantly lower in the GLUT4 deficient dorsal flexor muscle complex than in wild type littermates.

17. GLUT4 $4^{-1-}$ dorsal flexors exhibit a fibre type transition from fast-glycolytic fibres (IIB) to fast-oxidative fibres (IIA), which may partly cause the decrease in peak power and shortening velocities.

18. GLUT4 is essential for glycogen formation in the dorsal flexor complex since basal glycogen level is substantially decreased in GLUT4 deficient mice

19. The decreased glycogen level in GLUT4 $4^{-/}$dorsal flexors most likely contributes to increased fatigue susceptibility in the GLUT $4^{-/-}$muscle complex during a series of 150 shortening contractions.

20. The significant decrease in relative work output during the steady state phase of a contraction series of 150 shortening contractions indicates that energy supply via alternative routes is not capable to fully compensate for the lack of functional GLUT4. 


\section{7 - References}

1. Gorselink, M., Drost, M. R., Louw de, J., Willems, P. J. B., Rosielle, N., Janssen, J. D., and Van der Vusse, G. J. (2000) Accurate assessment of in situ isometric contractile properties of hind limb plantar and dorsal flexor muscle complex of intact mice. Pflügers Arch. 439, 665-670

2. Gorselink, M., Drost, M. R., Louw de, J., Willems, P. J. B., Dekkers, E. C. A., Janssen, J. D., and Vusse van der, G. J. In situ assessment of shortening and lengthening contraction properties of hind limb ankle flexors of intact mice. Pflügers Arch. in press

3. Brooks, S. V., and Faulkner, J. A. (1988) Contractile properties of skeletal muscle from young, adult and aged mice. J. Physiol. 404, 71-82

4. Brooks, S. V., and Faulkner, J. A. (1991) Forces and powers of slow and fast skeletal muscles in mice during repeated contractions. J. Physiol. 436, 701-710

5. Brooks, S. V., and Faulkner, J. A. (1994) Isometric, shortening, and lengthening contractions of muscle fiber segments from adult and old mice. Am. J. Physiol. 267, C507-C513

6. Close, R. (1965) Force: velocity properties of mouse muscles. Nature 206, 718-719

7. Faulkner, J. A., Jones, D. A., and Round, J. M. (1985) Injury to skeletal muscle of mice by lengthening contractions. $J$. Physiology $365,75 \mathrm{P}$

8. Haan de, A., Jones, D. A., and Sargeant, A. J. (1989) Changes in velocity of shortening, power output and relaxation rate during fatique of rat medial gastrocnemius muscle. Pflugers Arch. 413, 422-428

9. Phillips, S. K., and Woledge, R. C. (1992) A comparison of isometric force, maximum power and isometric heat rate as a function of sarcomere lenght in mouse skeletal muscle. Pflugers Arch. 420, 578-583

10. Warren, G. L., Hayes, D. A., Lowe, A., Williams, J. H., and Armstrong, R. B. (1994) Eccentric contraction-induced injury in normal and hindlimb-suspended mouse soleus and edl muscles. J. Appl. Physiol. 77, 1421-1430

11. Ashton-Miller, J. A., Veerichetty, Y. H. E., and Kadhiresan, A. (1992) an apparatus to measure in vivo biomechanical behavior of dorsi- and plantarflexors of mouse ankle. Pflugers Arch 72(3), 1205-1211

12. Bottinelli, R., Schiaffino, S., and Reggiani, C. (1991) Force-velocity relations and myosin heavy chain isoform compositions of skinned fibres from rat skeletal muscle. J. Physiol. (London) 437, 655-672

13. Burkholder, T. J., Fingado, B., Baron, S., and Lieber, R. L. (1994) Relationship between muscle fiber types and sizes and muscle architectural properties in the mouse hindlimb. J. Morphol. 221, 177-190

14. Walliman, T., Wyss, M., Brdiczka, D., and Nicolay, K. (1992) Intracellular compartmentation, structure and function of creatine kinase isoenzymes in tissues with high and fluctuating energy demands: the 'phosphocreatine circuit' for cellular energy homeostasis. Biochem. J. 281, 21-40

15. Wyss, M., Smeitink, J., Wevers, R. A., and Walliman, T. (1992) Mitochondrial creatine kinase: a key enzyme of aerobic energy metabolism. Biochim. Biophys. Acta $1102,119-166$

16. Nicolay, K., F.A., D. v., Reese, T., Kruiskamp, M. J., Gellerich, J. F., and Echteld, C. J. A. (1998) In situ measurements of creatine kinase flux by NMR. The lessons from bioengineered mice. Mol. Cell. Biochem. 184, 195-208

17. van Deursen, J., Heerschap, A., Oerlemans, F., Ruitenbeek, W., Jap, P., ter Laak, H., and Wieringa, B. (1993) Skeletal muscles of mice deficient in muscle creatine kinase lack burst activity. Cell 74, 621-631 
18. Steeghs, K., Oerlemans, F., de Haan, A., Heerschap, A., Verdoodt, L., de Bie, M., Ruitenbeek, W., Benders, A., Jost, C., van Deursen, J., Tullson, P., Terjung, R., Jap, P., Jacob, W., Pette, D., and Wieringa, B. (1998) Cytoarchitectural and metabolic adaptations in muscles with mitochondrial and cytosolic creatine kinase deficiencies. Mol. Cell Biochem. 184, 183-194

19. Steeghs, K., Benders, A., Oerlemans, F., Haan de, A., Heerschap, A., Ruitenbeek, W., Jost, C., Deursen van, J., Perryman, B., Pette, D., Bruckwilder, M., Koudijs, J., Jap, P., Veerkamp, J., and Wieringa, B. (1997) Altered $\mathrm{Ca}^{2+}$ responses in muscles with combined mitochondrial and cytosolic creatine kinase deficiencies. Cell 89, 93-103

20. Gorselink, M., Drost, M. R., Coumans, W. A., Kranenburg van, G. P. J., Hesselink, R. P., and Vusse van der, G. J. Impaired muscular contractile perfromance and adenine nucleotide handling in creatine kinase deficient mice. Am. J. Physiol. under revision

21. Westra, H. G., Haan de, A., Doorn van, J. E., and Haan de, J. (1990) IMP production and energy metabolism during exercise in rats in relation to age. Biochem. J. 239, 751755

22. Zeleznikar, R. J., Dzeja, P. P., and Goldberg, N. D. (1995) Adenylate kinase-catalyzed phosphoryl transfer couples ATP utilization with its generation by glycolysis in intact muscle. J Biol Chem 270, 7311-7319

23. Katz, E. B., Stenbit, A. E., Hatton, K., DePinho, R., and Charron, M. J. (1995) Cardiac and adipose tissue abnormalities but not diabetes in mice deficient in GLUT4. Nature 377, 151-155

24. Hesselink, M. K. C. (1998) Structural, functional and metabolic aspects of shortening and lengthening muscle contractions. In Movements Sciences p. 136, University Maastricht, Maastricht 
Chapter 9

154 


\section{Chapter 10}

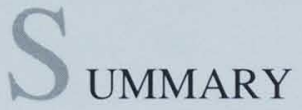

Summary of the thesis content. 


\section{1 - Summary}

Skeletal muscle comprises about $40 \%$ of the whole body mass, and plays an important role in locomotion. Animal models, like the rat and the mouse, are often used in studying muscular functioning and energy metabolism since experiments on these species are more easily to perform and to standardise compared to human studies on muscular functioning. Recently, the generation of transgenic and knockout mice has greatly enhanced the possibilities for studying the specific role of proteins and enzymes in energy metabolism and mechanical functioning of the skeletal muscle. Muscle contractile properties in mice, however, are mostly studied using invasive techniques, varying from single muscle fibre studies to whole muscle experiments. These invasive experimental procedures for studying skeletal muscle functioning in the mouse may, however, affect muscular function. Therefore, we wanted to investigate mechanical characteristics of skeletal muscle of the mouse under physiological conditions as much as possible.

Chapters 3 and 4 give a detailed description of the experimental model specifically developed for the assessment of the contractile properties under isometric as well as under dynamic conditions of the dorsal and plantar muscle complexes of the intact mouse. Isometric contractile performance of both muscle complexes can be assessed with the isometric mouse dynamometer (1) (Chapter 3). Using this measurement device, isometric muscle torque could be accurately measured within the range of $-14 \mathrm{mN} \cdot \mathrm{m}$ and $+14 \mathrm{mN} \cdot \mathrm{m}$. During the experiments, halothane anaesthetised Swiss wild-type mice were positioned on the thermostatic measurement platform, and fixated with a novel hip and foot fixation unit. Plantar or dorsal muscle complexes were electrically stimulated. For accurate measurements of muscle functioning in small animals, like mice, the following criteria are essential. First, stiff fixation of the animal to the measuring device is necessary to prevent displacement of bones. Any movements of bones due to loose fixation causes shortening of the muscle-tendon complex and, hence, influence the force generation. Second, the ankle axis should be aligned to the apparatus shaft as good as possible. In commonly used rigid knee fixation methods, this eccentricity will affect the measured muscle torque, because an additional fixation force will result in a non-muscle torque with a magnitude of the fixation force times the eccentricity distance. The novel fixation unit was evaluated by measuring knee and ankle displacements during contractions. The assessed ankle and knee displacement resulted in a calculated muscle fibre shortening of $2.5 \%$. The functional consequences of these hind limb movements were evaluated with a mathematical muscle model. Simulations of a contraction with this fibre shortening, using the mathematical muscle model showed only minor effects on maximal torque and the temporal parameters. 
Furthermore, we showed that muscle torque in our set-up is hardly affected by eccentricity between ankle and measurement axis. Measured tetanic muscle torques of intact dorsal and plantar flexors amounted to $3.2 \pm 0.4 \mathrm{mN} \cdot \mathrm{m}$ and $11.8 \pm 1.6 \mathrm{mN} \cdot \mathrm{m}$, respectively. The $1 / 2$-relaxation time of plantar flexors was significantly higher than in dorsal flexors ( $14.9 \pm 1.6 \mathrm{~ms}$ vs. $11.8 \pm 2.0 \mathrm{~ms})$.

Although the isometric dynamometer provides valuable information on muscle mechanical performance, it is not appropriate for determining contractile properties during shortening and lengthening contractions. Therefore, a mouse ergometer for assessment of shortening and lengthening contractile properties of intact plantar and dorsal flexors of the mouse has been described and evaluated (2) (Chapter 4). With the mouse ergometer, muscular torque ranging from -50 to $50 \mathrm{mN} \cdot \mathrm{m}$ can be assessed. Evaluation of the fixation procedure of the animal to the set-up via 3-D monitoring of the muscle-tendon complex length showed that the additional shortening in length due to a contraction with maximal torque output exerts only minor effects on assessed torque. Furthermore, it was found that a misalignment between the axis of rotation of the set-up relative to the axis of rotation in the ankle joint, i.e., eccentricity, during a routine experiment was estimated to be less than $1.0 \mathrm{~mm}$, and hence, did not influence the measured torque output in our experimental set-up. Peak power per unit muscle mass (mean $\pm \mathrm{sd}$ ) of intact dorsal and plantar flexors amounted to $0.27 \pm 0.02 \mathrm{~W} \cdot \mathrm{g}^{-1}$ and $0.19 \pm 0.03 \mathrm{~W} \cdot \mathrm{g}^{-1}$, respectively. The angular velocity at maximal peak power generated by the dorsal flexor complex and the plantar flexor complex amounted to $1100 \pm 190^{\circ} \cdot \mathrm{s}^{-1}$ and $700 \pm 90^{\circ} \cdot \mathrm{s}^{-1}$, respectively.

In the present thesis, we also aimed to study the consequence of defects in energy metabolism on mechanical functioning of murine skeletal muscle. First, we studied the effect of disruption of the cytoplasmic and mitochondrial creatine kinase genes on muscular performance. Creatine kinase (CK) forms a small family of isoenzymes playing an important role in maintaining the concentration of ATP and ADP in muscle cells. To delineate the impact of lack of CK activity, we studied contractile performance during a single maximal tetanic contraction and during 12 repeated tetanic contractions of intact dorsal flexors of CK knockout mice (3) (chapter 5). To investigate the effect on ATP regeneration, muscular high-energy phosphate content was determined at rest, immediately after the contraction series and after a 60 seconds recovery period. Maximal torque of the dorsal flexors was significantly lower in $\mathrm{CK}^{-1-}$ mice than in wild type animals, i.e., $23.7 \pm 5.1$ and $33.3 \pm 6.8 \mathrm{mN} \cdot \mathrm{m} \cdot \mathrm{g}^{-1}$ wet weight, respectively. Lower muscle ATP $\left(20.1 \pm 1.4\right.$ in $\mathrm{CK}^{-1-}$ vs. $28.0 \pm 2.1 \mu \mathrm{mol} \cdot \mathrm{g}^{-1}$ dry weight in controls) and higher IMP $\left(1.2 \pm 0.5\right.$ in $\mathrm{CK}^{-1-}$ vs. $0.3 \pm 0.1 \mu \mathrm{mol} \cdot \mathrm{g}^{-1} \mathrm{dry}$ weight in controls) levels, at the onset of contraction, may contribute to the declined contractility in $\mathrm{CK}^{--}$mice, but do not play a decisive role. 
In contrast to wild type muscles, ATP levels could not be maintained during the series of 12 tetanic contractions of dorsal flexors of $\mathrm{CK}^{-1}$ mice and dropped to $15.5 \pm 2.4$ $\mu \mathrm{mol} \cdot \mathrm{g}^{-1}$ dry weight. The significant increase in tissue IMP $\left(2.4 \pm 1.1 \mu \mathrm{mol} \cdot \mathrm{g}^{-1}\right.$ dry weight) content following the contraction series indicates ATP regeneration through adenylate kinase, which activity is inherently not capable to fully compensate for the lack of CK. ATP regeneration via the adenylate kinase pathway is a likely cause of reduced basal adenine nucleotide levels in $\mathrm{CK}^{-/}$mice.

In chapter 5 , we observed that during a series of 12 consecutive high-intensity contractions, $\mathrm{CK}$ deficient $\left(\mathrm{CK}^{-/}\right)$dorsal flexors could not maintain their ATP content at the pre-contraction level, and was accompanied with a substantial increase in inosine monophosphate (IMP). In chapter 6 it was, therefore, assumed that during a second series of contractions, $\mathrm{CK}^{-/-}$muscles would further deplete their muscular ATP pool with a concomitant rise in tissue IMP, which would unfavourably affect mechanical performance. Our data, however, indicate that tissue ATP $(17.7 \pm 3.1$ $\left.\mu \mathrm{mol} \cdot \mathrm{g}^{-1}\right)$ did not further decline and IMP did not further increase $(1.2 \pm 0.4,2.4 \pm 1.1$, and $2.5 \pm 1.0 \mu \mathrm{mol} \cdot \mathrm{g}^{-1}$ for resting, end of first series and end of second series, respectively) during the second series of contractions in $\mathrm{CK}^{-/-}$muscles. Moreover, the mechanical performance of $\mathrm{CK}^{-/}$dorsal flexors was comparable during the two contraction series. The fact that the IMP content did not further increase in combination with a stable ATP level during the second contraction series, strongly suggest that AMP produced by the catalytic action of adenylate kinase is not degraded to IMP, but may act as intracellular high-energy phosphate carrier between for instance, ATP produced in the glycolytic pathway and the site of ATP consumption, according to the theory of Zeleznikar and co-workers (4). Surprisingly, the decline in torque during the second contraction series of wild type muscles $(-20.1 \%)$ was more pronounced than during the first series $(-12.3 \%)$, which was accompanied with a substantial increase in IMP content $\left(0.3 \pm 0.1,0.2 \pm 0.1\right.$, and $2.6 \pm 2.1 \mu \mathrm{mol} \cdot \mathrm{g}^{-1}$ for resting, end of first series and end of second series, respectively). The latter findings indicate that AMP deaminase, in combination with the adenylate kinase pathway, is operational during the second, but not during the first series of contractions in wild type muscles.

GLUT4 plays a predominant role in glucose uptake during muscular contraction $(5,6)$. In the present study, we have investigated in mice whether disruption of the GLUT4 gene affects isometric and shortening contractile performance of the dorsal flexor muscle complex in situ (chapter 8). Moreover, we have explored the hypothesis that lack of GLUT4 enhances muscular fatigability. Isometric performance normalised per unit muscle mass during a single tetanic contraction did not differ between wild type and GLUT4 $4^{-1-}$ mice. 
Shortening contractions, however, revealed a significant 1.4 fold decrease in peak power per unit mass, most likely caused by the fibre type transition from fastglycolytic fibres (IIB) to fast-oxidative fibres (IIA) in GLUT4 $^{-/}$dorsal flexors. Moreover, it can be concluded that GLUT4 is essential for glycogen formation in the dorsal flexor complex since basal glycogen level is substantially decreased in GLUT4 deficient mice. The decreased glycogen level in GLUT4 ${ }^{-/}$dorsal flexors most likely contributes to increased fatigue susceptibility in $\mathrm{GLUT}^{-/-}$mice. The significant decrease in relative work output during the steady state phase indicates that energy supply through compensatory routes is not capable to fully compensate for the lack of GLUT4.

The thesis ends with a general discussion, summarising the major findings in the preceding chapters, with special reference to the assessment of muscular performance in the intact mouse. Moreover, future perspectives for the use of the newly developed experimental models are presented. 


\section{2 - References}

1. Gorselink, M., Drost, M. R., Louw de, J., Willems, P. J. B., Rosielle, N., Janssen, J. D., and Van der Vusse, G. J. (2000) Accurate assessment of in situ isometric contractile properties of hind limb plantar and dorsal flexor muscle complex of intact mice. Pflügers Arch. 439, 665-670

2. Gorselink, M., Drost, M. R., Louw de, J., Willems, P. J. B., Dekkers, E. C. A., Janssen, J. D., and Vusse van der, G. J. In situ assessment of shortening and lengthening contraction properties of hind limb ankle flexors of intact mice. Pflügers Arch. in press

3. Steeghs, K., Benders, A., Oerlemans, F., Haan de, A., Heerschap, A., Ruitenbeek, W., Jost, C., Deursen van, J., Perryman, B., Pette, D., Bruckwilder, M., Koudijs, J., Jap, P., Veerkamp, J., and Wieringa, B. (1997) Altered $\mathrm{Ca}^{2+}$ responses in muscles with combined mitochondrial and cytosolic creatine kinase deficiencies. Cell 89, 93-103

4. Zeleznikar, R. J., Dzeja, P. P., and Goldberg, N. D. (1995) Adenylate kinase-catalyzed phosphoryl transfer couples ATP utilization with its generation by glycolysis in intact muscle. J Biol Chem 270, 7311-7319

5. Brozinick, J. T., Yaspelkis, B. R., Wilson, C. M., and Grant, K. E. (1996) Glucose transport and GLUT4 protein distribution in skeletal muscle of GLUT4 transgenic mice. Biochem. J. 313, 133-140

6. Klip, A., Volchuk, A., He, L., and Tsakidiris, T. (1996) The glucose transporter of skeletal muscle. Cell Dev. Biol. 7, 229-237 


\section{Chapter 11}

AMENVATTING

Samenvatting van het proefschrift 


\section{1 - Samenvatting}

Ongeveer $40 \%$ van de totale massa van het lichaam wordt ingenomen door de skeletspieren. Deze skeletspieren spelen met name een belangrijke rol bij het bewegen van de afzonderlijke deelsegmenten van het lichaam. Het functioneren van de skeletspieren wordt door vele facetten bepaald, zoals de mechanische eigenschappen, het energie metabolisme en de aansturing van de spieren. Om de skeletspier functie en het energie metabolisme van de skeletspier te bestuderen, wordt meestal gebruik gemaakt van proefdier modellen, zoals de rat en de muis. De reden hiervoor is dat experimenten met deze knaagdieren, vergeleken met de condities van de experimenten die bij de mens worden uitgevoerd, eenvoudiger zijn. Recent is de muis als dier experimenteel model nog aantrekkelijker geworden, omdat er de mogelijkheid bestaat om transgene en knock-out muismodellen te genereren. Deze muizen bieden de mogelijkheid om de rol van specifieke eiwitten en enzymen in het energie metabolisme en de contractiele eigenschappen van de skeletspier in detail te bestuderen. Mechanische karakteristieken van een spiercontractie van de muis worden momenteel met behulp van invasieve in vitro technieken bepaald. Deze experimentele condities kunnen de skeletspierfunctie echter negatief beïnvloeden. Het hoofddoel van dit project was daarom het ontwikkelen en evalueren van een meetmethode om de skeletspier functie van de muis in zijn natuurlijke omgeving te onderzoeken.

De hoofdstukken 3 en 4 geven een gedetailleerde beschrijving van het ontwikkelde model, dat de mogelijkheid biedt om de contractie eigenschappen van het kuitspiercomplex en het scheenbeenspiercomplex van de muis, onder zowel isometrische als dynamische omstandigheden, te bestuderen. De isometrische eigenschappen van beide spiercomplexen kunnen met behulp van de isometrische dynamometer in kaart gebracht worden (1) (hoofdstuk 3). Het isometrische spierkoppel kan met behulp van dit onderdeel van het experimentele model in het bereik van -14 $\mathrm{mN} \cdot \mathrm{m}$ en $14 \mathrm{mN} \cdot \mathrm{m}$ bepaald worden. Tijdens een experiment wordt de muis met behulp van halothaan verdoofd, en via de heup en voet aan de meetapparatuur bevestigd. De beide spiercomplexen kunnen nu geactiveerd worden via elektrische stimulatie van de zenuwen, die de desbetreffende spiercomplexen innerveren. Om nauwkeurige functiemetingen aan de skeletspier van muizen uit te voeren, zijn de volgende criteria van essentieel belang. Ten eerste moet de muis aan de meetapparatuur gefixeerd worden, zodat bewegingen van de achterpoot voorkomen worden. Deze bewegingen zullen namelijk extra verkortingen van het spierpeescomplex opleveren, die de krachtopbouw verstoren. Daarnaast moeten de draaias van het enkelgewricht en de as van de meet apparatuur zoveel mogelijk in elkaars verlengde liggen. 
Bij de meest gebruikte kniefixatie methodes is het praktisch niet te voorkomen dat er een afwijking optreedt tussen de draaias van het enkelgewricht en de as van de meetapparatuur (excentriciteit). Het gemeten spierkoppel wordt hierdoor verstoord omdat een extra niet-spierkoppel via het onderbeen op de meetas wordt overgedragen. De nieuw ontwikkelde fixatiemethode is geëvalueerd via metingen van de bewegingen van het knie- en enkelgewricht tijdens een maximale spiercontractie. Deze bewegingen leiden tot een geschatte spiervezel verkorting van ongeveer $2.5 \%$. De consequentie van deze extra verkorting zijn bepaald via een wiskundig spiermodel. Simulaties van een isometrische spiercontractie met deze extra vezelverkorting, resulteerde in minimale verschillen in maximaal koppel, snelheid van krachtopbouw en relaxatie vergeleken met een simulatie zonder deze extra verkorting. Uit deze simulaties is geconcludeerd dat de nieuw ontwikkelde fixatie procedure geschikt is om spiereigenschappen van de intacte muis tijdens isometrische contracties op betrouwbare manier te meten. Daarnaast blijkt dat een excentriciteit tussen het enkelgewricht en de as van de meetapparatuur in onze experimentele opstelling geen effect heeft op het isometrische spierkoppel. Het gemeten spierkoppels van de intacte scheenbeenspier- en kuitspiercomplexen waren respectievelijk $3,2 \pm 0,4 \mathrm{mN} \cdot \mathrm{m}$ en 11,8 $\pm 1,6 \mathrm{mN} \cdot \mathrm{m}$. De 1/2-relaxatie tijd van het kuitspier complex was significant hoger dan de $1 / 2$-relaxatietijd van het scheenbeenspier complex $(14,9 \pm 1,6 \mathrm{~ms}$ versus $11,8 \pm 2,0$ $\mathrm{ms}$ ).

Alhoewel de isometrische eigenschapen van de beide spiercomplexen met de isometrische dynamometer bepaald kunnen worden, kunnen de karakteristieken van spiercontracties tijdens verkorting en verlenging van de spier met dit apparaat niet onderzocht worden. Daarom is het experimentele model uitgebreid met de muizenergometer. Hiermee kunnen de spier karakteristieken tijdens verkorting en verlenging bepaald worden (2) (hoofdstuk 4). Met behulp van de muizenergometer kunnen spierkoppels van verkortende en verlengende contracties in een bereik van -50 tot $50 \mathrm{mN} \cdot \mathrm{m}$ bepaald worden. De fixatie van de muis aan de meetapparatuur is getest via het meten van veranderingen in de lengte van het spierpeescomplex tijdens een verkortende contractie van het kuitspier complex. De resultaten tonen aan dat het spierkoppel nauwkeurig gemeten kan worden, terwijl de snelheden van krachtopbouw en relaxatie minder nauwkeurig bepaald kunnen worden. De excentriciteit tussen de meetas en het enkelgewricht tijdens de experimenten zijn nooit groter dan 1,0 mm. Een excentriciteit van deze grootte heeft in onze experimentele set-up geen invloed op het gemeten spierkoppel. 
Het maximale vermogen, genormaliseerd voor de spier massa, van het kuitspier- en scheenbeenspiercomplex was respectievelijk $0,27 \pm 0,02 \mathrm{~W} \cdot \mathrm{g}^{-1}$ en $0,19 \pm 0,03 \mathrm{~W} \cdot \mathrm{g}^{-1}$. De hoeksnelheden behorende bij deze vermogens waren $1100 \pm 190$ en $700 \pm 90$ graden per seconde.

Een tweede doel van dit proefschrift was het bepalen van de effecten van afwijkingen in het energie metabolisme op het functioneren van de intacte skeletspier. Ten eerste hebben we het effect van creatine kinase deficiëntie $\left(\mathrm{CK}^{-\prime}\right)$ op het functioneren van de spier bestudeerd. Er zijn verschillende isovormen van creatine kinase in de skeletspiercel aanwezig, die tezamen een belangrijke rol spelen in de regulatie van de cellulaire ATP en ADP concentraties. De effecten van CK deficiënties zijn in het scheenbeenspier complex bestudeerd tijdens een enkelvoudige maximale spiercontractie en tijdens een serie van 12 maximale contracties (hoofdstuk 5). De hoeveelheid energierijke fosfaten in rust, direct na 12 contracties en 60 seconden na de contractie serie zijn bepaald om de gevolgen van CK deficiëntie te bepalen op het ATP regenererende vermogen van de skeletspiercel tijdens deze vorm van inspanning. Het maximale koppel van het scheenbeenspiercomplex was significant lager in $\mathrm{CK}$ deficiënte muizen dan in normale muizen, namelijk $23,7 \pm 5,1$ versus $33,3 \pm 6,8 \mathrm{mN} \cdot \mathrm{m}$ per gram nat spiergewicht. Een lager ATP $\left(20,1 \pm 1,4\right.$ in $\mathrm{CK}^{-/}$versus $28,0 \pm 2,1 \mu \mathrm{mol}$ per gram droog gewicht) en een hoger inosine monofosfaat (IMP) $\left(1,2 \pm 0,5\right.$ in $\mathrm{CK}^{-/}$ versus $0,3 \pm 0,1 \mu \mathrm{mol}$ per gram droog gewicht) gehalte in rust, zou kunnen bijdragen aan de daling van het maximale spierkoppel in $\mathrm{CK}^{-/}$muizen. Uit de resultaten van hoofdstuk 6 blijkt echter dat deze biochemische veranderingen niet de hoofdoorzaak kunnen zijn van de verminderde mechanische output. De scheenbeenspieren van $\mathrm{CK}^{-1-}$ muizen konden, in tegenstelling tot normale muizen, hun ATP niveau tijdens de contractieserie niet constant houden. Het ATP gehalte daalde tot $15,5 \pm 2,4 \mu \mathrm{mol}$ per gram droog gewicht. De toename in IMP $(2,4 \pm 1,1 \mu \mathrm{mol}$ per gram droog gewicht) tijdens de contractie serie duidt erop dat de ATP regeneratie via adenylaat kinase plaats vindt. Dit systeem was echter niet instaat om de afwezigheid van CK volledig te compenseren. De ATP regeneratie via het adenylaat kinase systeem resulteert daarnaast ook in een daling van het niveau van de adenine nucleotiden in $\mathrm{CK}$ deficiënte scheenbeenspieren.

Omdat we in hoofdstuk 5 hebben we laten zien dat tijdens een serie contracties van hoge intensiteit, de CK deficiënte muis niet in staat is zijn niveau van ATP te handhaven, terwijl het gehalte aan IMP significant toenam, hebben wij in hoofdstuk 6 verondersteld dat tijdens een tweede inspanningsserie het ATP gehalte verder zal dalen en het IMP gehalte verder zou stijgen. Deze biochemische veranderingen zouden het mechanisch functioneren van de skeletspier in CK deficiënte muizen negatief kunnen beïnvloeden. 
De resultaten lieten echter zien dat het ATP $(17,7 \pm 3,1 \mu \mathrm{mol}$ per gram droog gewicht) en IMP gehalte $(1,2 \pm 0,4,2,4 \pm 1,1$ en $2,5 \pm 1,0 \mu$ mol per gram droog gewicht tijdens respectievelijk rust, direct na de eerste contractieserie en direct na de tweede contractieserie) constant bleef tijdens de tweede contractieserie. Het mechanisch functioneren van de $\mathrm{CK}^{-/}$spieren in de tweede contractieserie was vergelijkbaar met die tijdens de eerste contractieserie. Het feit dat het IMP gehalte niet verder steeg in combinatie met een stabiel ATP gehalte, duidt erop dat AMP, in plaats van substraat voor IMP vorming, dienst doet als transport mechanisme voor de energierijke fosfaten, wat in overeenstemming is met de theorie van Zeleznikar en collega's (3). Opvallend was dat in de scheenbeenspieren van normale muizen de daling in spierkoppel tijdens de tweede contractie serie sterker was dan in de eerste contractie serie. De sterkere daling van het spierkoppel tijdens de tweede contractie serie werd vergezeld van een stijging in het IMP gehalte $(0.3 \pm 0.1,0.2 \pm 0.1$ en $2.6 \pm 2.1 \mu \mathrm{mol}$ per gram droog gewicht tijdens respectievelijk rust, direct na de eerste contractie serie en direct na de tweede contractie serie). Deze bevindingen duiden erop dat in de scheenbeenspieren van normale muizen, het AMP deaminase in combinatie met adenylaat kinase wel actief is tijdens de tweede contractie serie maar niet gedurende de eerste contractie serie.

Tijdens spiercontractie speelt het GLUT4 eiwit een belangrijke rol in de opname van glucose in de spiercel $(4,5)$. In hoofdstuk 8 hebben we bestudeerd of muizen met een GLUT4 deficiëntie in de skeletspieren een afwijkend skeletspier functioneren hebben tijdens isometrische en verkortende contracties. Daarnaast hebben we in dit hoofdstuk de hypothese getoetst dat Glut4 deficiëntie de gevoeligheid voor vermoeidheid van de spieren doet toenemen. Het functioneren tijdens een isometrische contractie verschilt echter niet tussen scheenbeenspieren deficiënt in GLUT4 vergeleken met normale spieren. Het maximale vermogen tijdens verkortende contracties was significant gedaald in GLUT4 deficiënte scheenbeenspieren. Deze daling in maximaal vermogen wordt waarschijnlijk veroorzaakt door een gedeeltelijke verschuiving in spiervezelsamenstelling, met name van snelle glycolytische vezels (IIB) naar snelle oxidatieve vezels (IIA). Uit het verminderde glycogeengehalte in GLUT4 deficiënte scheenbeenspiercomplexen in rust, kan geconcludeerd worden dat GLUT4 waarschijnlijk een essentiële rol speelt in de glycogeenvorming. Deze daling in glycogeen speelt waarschijnlijk een belangrijke rol in de toegenomen vatbaarheid voor vermoeidheid in de spieren deficiënt in GLUT4. 
Mijn proefschrift eindigt met een algemene discussie, waarin de belangrijkste bevindingen kort worden samengevat. Hierbij is speciaal aandacht besteed aan het ontwikkelde model om skeletspierfunctie in intacte muizen te meten. Daarnaast is een korte beschouwing omtrent de mogelijke verbeteringen van het experimentele model gegeven en toekomstige toepassingsmogelijkheden toegelicht. 


\section{2 - Referenties}

1. Gorselink, M., Drost, M. R., Louw de, J., Willems, P. J. B., Rosielle, N., Janssen, J. D., and Van der Vusse, G. J. (2000) Accurate assessment of in situ isometric contractile properties of hind limb plantar and dorsal flexor muscle complex of intact mice. Pflügers Arch. 439, 665-670

2. Gorselink, M., Drost, M. R., Louw de, J., Willems, P. J. B., Dekkers, E. C. A., Janssen, J. D., and Vusse van der, G. J. In situ assessment of shortening and lengthening contraction properties of hind limb ankle flexors of intact mice. Pflügers Arch. in press

3. Zeleznikar, R. J., Dzeja, P. P., and Goldberg, N. D. (1995) Adenylate kinase-catalyzed phosphoryl transfer couples ATP utilization with its generation by glycolysis in intact muscle. J Biol Chem 270, 7311-7319

4. Brozinick, J. T., Yaspelkis, B. R., Wilson, C. M., and Grant, K. E. (1996) Glucose transport and GLUT4 protein distribution in skeletal muscle of GLUT4 transgenic mice. Biochem. J. 313, 133-140

5. Klip, A., Volchuk, A., He, L., and Tsakidiris, T. (1996) The glucose transporter of skeletal muscle. Cell Dev. Biol. 7, 229-237 


\section{Publications}

\section{PAPERS (accepted and under revison)}

1. M. Gorselink, M.R. Drost, J. de Louw, P.J.B. Willems, N. Rosielle, J.D. Janssen, G.J. van der Vusse (2000) Accurate assessment of in situ isometric contractile properties of hindlimb plantar and dorsal flexor muscle complex of intact mice. Eur. J. Physiol. 439: $665-670$.

2. M. Gorselink, M.R. Drost, J., de Louw de, P.J.B., Willems, M.K.C. Hesselink, E.C.A. Dekkers, G.J. van der Vusse (2001) In situ assessment of shortening and lengthening contraction properties of hindlimb ankle flexors of intact mice. Eur. J. Physiol., in press.

3. M. Gorselink, M.R. Drost, Coumans, W.A., Kranenburg, G.P.J. van, Hesselink, R.P., Vusse, G.J. van der. (2001) Impaired muscular contractile performance and adenine nucleotide handling in creatine kinase deficient mice. Am. J. Physiol, in press.

4. M. Gorselink, M. R. Drost, G. J. van der Vusse (2001) Murine muscles deficient in creatine kinase tolerate consecutive series of high-intensity contractions. Eur. J. Physiol. under revision.

\section{PAPERS (submitted)}

5. M. Gorselink, M.R. Drost, K.F.J. de Brouwer, G. Schaart, G.P.J. van Kranenburg, T.H.M. Roemen, M. van Bilsen, M. J. Charron, and G.J. van der Vusse (2001) Increased muscle fatigability in GLUT4 deficient mice. Am. J. Physiol. submitted

6. R.P. Hesselink, M. Gorselink, G. Schaart, A.J.M. Wagenmakers, J. Kamphoven, A.J.J. Reuser, G.J. van der Vusse, M.R. Drost (2001) Impaired performance of skeletal muscle in $\alpha$-glucosidase knockout mice. Am. J. Physiol. submitted.

7. B. Oosthuyse, L. Moons, H. Beck, J. van Dorpe, M. Gorselink, D. Nuyens, S. Heymans, G. Theilmeier, M. Dewerchin, V. Laudenbach, P. Vermylen, T. Acker, A. Damert, N. Cashman, H. Fujisawa, M. R. Drost, W. Robberecht, R. Sciot, F. Bruyninckx, P. Gressens, D. Collen, P. Carmeliet (2000) Targeted deletion of the hypoxia-response element in the VEGF promotor causes adult motor neuron degeneration due to impaired $\mathrm{VEGF}_{165} /$ neuropilin-I neuroprotection. Nature Genetics, submitted. 


\section{BOOK CHAPTERS}

1. M. Gorselink, M.R. Drost, G.J. van der Vusse (2001) Muscle morphology, metabolism and muscle function. General Principles. In: Nutrition and metabolism in Respiratory Diseases. Eur. Res. Monograph, A. Rossi, Verona, in press.

\section{ABSTRACTS}

1. M. Gorselink, M.R. Drost, G.J. van der Vusse (1999) The effect of creatine kinase deficiency on in vivo functioning of mice dorsal flexors. J. Mol. Cell. Cardiol. 31(6): sa11.

2. M. Gorselink, M. Drost, G.J. van der Vusse (1999) The effect of creatine kinase deficiency on in vivo mechanical functioning of mice skeletal muscle. Eur. J. Physiol.438: R70.

3. R.P. Hesselink, M. Gorselink, A.J.M. Wagenmakers, M.R. Drost (1999) Characterisation of isometric muscle contractions of $\alpha$-glucosidase knockout mice. J. Mus. Res. Cell Motil. 20: 857.

4. M. Gorselink, M.R. Drost, G.J. van der Vusse (1999) The effect of creatine kinase on mechanical functioning of mice skeletal muscle. J. Mus. Res. Cell Motil. 20: 856.

5. M. Gorselink, M.R. Drost, G.J. van der Vusse (2001) In situ assessment of shortening and lengthening properties in hind limb flexors of intact mice. J. Mus. Res. Cell Motil., in press.

6. R.P. Hesselink, G. Schaart, M. Gorselink, J. Kamphoven, A.J.M. Wagenmakers, G.J. van der Vusse, M.R. Drost (2001) Cytoskeletal changes and impaired isokinetic muscle performance in $\alpha$-glucosidase knockout mice. J. Mus. Res. Cell Motil. In press. 


\section{Curriculum Vitae}

Marchel Gorselink werd geboren op 24 juni 1972 te Oene (Gelderland). In 1990 behaalde hij het VWO diploma aan de Scholengemeenschap De Heertganck te Heerde. Aansluitende studeerde hij Gezondheidswetenschappen aan de Universiteit Maastricht (UM) met als afstudeerrichting Bewegingswetenschappen, die gevolgd werd door de afstudeerrichting Biologische Gezondheidskunde. In juli 1996 behaalde hij zijn doctoraal diploma, na afstudeerstages bij dr. H. Keizer, capaciteitsgroep bewegingswetenschappen en prof. dr. R. Mensink, capaciteitsgroep humane biologie. In oktober 1996 startte hij als assistent in opleiding (AIO) onder leiding van prof. dr. G.J. van der Vusse (fysiologie, UM), prof. dr. ir. J.D. Janssen (BMT, TU/e, Eindhoven) en dr. ir. M.R. Drost (bewegingswetenschappen, UM) bij de capaciteitsgroep fysiologie, geneeskunde aan de Universiteit Maastricht. Vanaf 1 februari 2001 is hij als onderzoeker werkzaam bij Numico-Research te Wageningen. 


\section{Dankwoord}

Het is bijna af, nu alleen nog het dankwoord. Eigenlijk is dit één van de meest moeilijke onderdelen van het proefschrift, met name het feit dat je zeker niet iemand moet vergeten. Daarom wil ik gebruik maken van een handige noviteit recent geïntroduceerd door een mede-AIO: de alfabetische dankwoordenlijst. Via deze noviteit kan ik dan ook met de meest belangrijke persoon van de afgelopen jaren beginnen en hoop ik verder dat ik niemand zal vergeten.

Berg van den, Merel: Merel, ik wil je bedanken voor de steun die ik heb gekregen gedurende de afgelopen periode. De gespreken met jou over allerlei niet proefschrift zaken hebben mij een heel andere visie ten-aanzien van het AIO-schap gegeven. Mede hierdoor is het dan ook mogelijk geweest om zo nu en dan wat meer tijd in mijn proefschrift te steken dan eigenlijk goed is. Ik denk dat, na afronding van mijn proefschrift, de komende jaren alleen nog maar mooier zullen worden.

Brands, Desiree: Desiree ik wil je bedanken voor alle steun en het op de hoogte houden van alle weetjes rondom de vakgroep bewegingswetenschappen.

Bolhuis, Annette: Ondanks het feit dat we het wetenschappelijk gezien niet altijd eens waren, was je toch een prettige kamergenoot en heb ik een aantal wijze levenslessen opgestoken.

Borghouts, Lars: Samen gestart binnen de studie bewegingswetenschappen via vele feesten, wederom bijeen gekomen als AIO binnen de vakgroep bewegingswetenschappen Lars, ik wil je bedanken voor alle kritische en gezellige momenten van de afgelopen jaren.

Collega's: Hierbij wil ik alle collega's bedanken voor hun steun gedurende de afgelopen jaren; Jos Adam, Marc van Bilsen, Claire Bollen, Eric van Breda, Hans Degens, Jan Glatz, Fred Hartgens, Gerard Houben, Frans van Nieuwenhoven, Theo Roemen, Frans Verstappen, Harry Wandler, Jodil Willems, Peter Willems en de overige collega's van de vakgroepen fysiologie, bewegingswetenschappen en biomedische technologie.

Collega AIO's: Ik wil al mijn mede-(oud)AIO's bedanken voor hun kritische en gezellige steun: Annette, Danny, Debbie, Freddy, Gerard, Joep, Karin, Kristel, Lars, Luc, Maike, Marielle, Mascha, Miriam, Reinout, René, Ron, Yvonne.

Coumans, Will: Ik wil je bedanken voor je ondersteuning tijdens de analyses van de energierijke fosfaat niveaus via de HPLC methode. 
Design Arbeid: Ruben bedankt voor de gezelligheid en voor het ontwerpen van de voorkant van dit proefschrift.

Drost, Maarten: Maarten, een betere begeleider had ik me niet kunnen wensen. Met name de kritische, soms wat chaotische, gesprekken en experimenten zijn voor mij noodzakelijk geweest om de technische en biologische aspecten gedurende mijn AIO periode te integreren.

Donkelaar, René: Je bent nu alweer een tijdje AIO af, toch wil ik je bedanken voor de steun en gezelligheid gedurende de vele volleybal-, AIO- en kroeguurtjes die we hebben door gebracht. Misschien moeten we binnenkort, samen met Thijs, maar weer eens yoghurt met zwoerd gaan eten.

Dekkers, Erwin: Bedankt voor de technische ondersteuning van het ontwerp van zowel de muizen isometrische dynamometer en muizen ergometer.

Familie Gorselink: Eigenlijk hoef ik hier niks te zeggen, maar goed. Pa, ma en Kristel bedankt voor alle steun.

Familie Van den Berg: Jacques, Trees, Martijn, Mijke en Menno, bedankt dat ik jullie dochter of zus(je) mag lenen.

Hesselink, Matthijs: Tja, jij loopt de laatste jaren als een rode draad door mijn studie en AIO periode. Ik ben mijn stage begonnen bij jou en Hans. Gedurende deze stage heb ik me bezig gehouden met de beroemde denervatie studies. Uiteindelijk ben ik AIO bij fysiologie geworden, en gedurende deze periode heb je me ook altijd zeer kritisch gesteund. Nu ik bij Numico-Reseach werk, denk ik af en toe nog wel eens:" hoe zou Matthijs dat doen...". Matthijs ik wil je bedanken voor alle hulp en gezelligheid van de afgelopen jaren.

Hesselink, Reinout: Junior, ik heb je de laatste jaren pas goed leren kennen, en ik denk dat je een meer dan waardig opvolger bent. Ik draag de muizen apparatuur dan ook graag aan je over.

Hon de, Olivier: Eigenlijk, net als ik, geen officieel lid van de vakgroep bewegingswetenschappen, vandaar waarschijnlijk ook onze verbondenheid. Daarnaast natuurlijk ook de enorme passie voor onze Godenzonen. Jij bent nu werkzaam bij het Necedo en ik hoop dat we nog veel contact zullen houden.

Janssen, Jan: Jan, ik wil je bedanken voor alle steun en zonder jou was dit project waarschijnlijk nooit van de grond gekomen. 
Keulen, Ron: Pas in een later stadium bij de vakgroep gekomen, en tijdens mijn afscheidsavond er achter gekomen dat je erg gezellige kan stappen. Bedankt voor alle goede gesprekken.

Kolenburg, Leon: Ontzettend veel lol gehad tijdens de vele volleybal kroeg en woensdagmiddag uurtjes. Ik denk dat we elkaar nog regelmatig spreken, maar toch bedankt ouwe Wieckser.

Keizer, Hans: $\mathrm{Bij}$ jou ben ik mijn wetenschappelijke carrière begonnen, en jou onuitputbaar enthousiasme heb ik (nog steeds) als een enorme stimulans ervaren. Bedankt voor alle ondersteuning en gezelligheid.

Kranenburg, Gerrit: Tja, waar zal ik beginnen. Jij bent iemand de eigenlijk de hele vakgroep bij elkaar houdt, met name dankzij jou onuitputbare kennis op het gebied van auto's, wasmachines badkamers, keukens, kikkers etc. Daarnaast je ondoorgrondbare manier van het bepalen de eigenschappen van allerlei sleutelenzymen heeft bijgedragen aan het tot stand komen van mijn proefschrift.

Kuipers, Harm: Harm, bedankt voor alle steun en voor het kritisch doorlezen van mijn proefschrift.

Leescommissie: Ik wil alle leden van de beoordelingscommissie (prof. dr. H. Kuipers, prof. dr. ir. M.G.J Arts, dr. A. J. M. Wagenmakers, Prof. dr. B. Wieringa, dr. ir. W. C. Oomens) bedanken voor het kritisch doorlezen van dit proefschrift.

Loon, Luc: Luc, ik wil je bedanken voor, de soms onnavolgbare, gesprekken over allerlei, ook niet-wetenschappelijke, zaken.

Paranimfen: Ik wil mijn beide paranimfen Bas en Anke bij deze alvast bedanken voor hun steun tijdens de dichterbij komende promotie.

Pijnappels, Miriam: Pimmetje, je bent niet helemaal tot je recht gekomen in Maastricht, maar volgens mij ben je goed terechtgekomen in Amsterdam. Verder moet ik nog regelmatig terug denken aan ons Matlab gestuntel.

Rietjens, Gerard: Gerard, jij bent naast een goede collega altijd een goede vriend geweest, die altijd klaar stond wanneer ik je nodig had. 
Schaart, Gert: Gert, ik wil je bedanken voor alle steun en gezelligheid de afgelopen 2 jaren. Met name je kennis en vaardigheid zou ik graag wat eerder in mijn AIO periode zijn tegen gekomen. Daarnaast wil ik natuurlijk ook Helma bedanken voor mijn eerste stappen in de wereld van de Westen blot.

Vusse van der, Ger: Ger, je inzet, enthousiasme, inzicht en met name je onuitputtelijke kennis hebben enorm bijgedragen bij het tot stand komen van dit proefschrift. Ik hoop dat we in de toekomst onze samenwerking kunnen voortzetten.

Willems, Paul: Tja, Ajax vs. Roda, het zal altijd een strijd blijven. Paul bedankt voor de ondersteuning van de afgelopen jaren en natuurlijk voor de gezellige (voetbal)gesprekken.

Het dankwoord is toch nog langer geworden dan ik dacht, en ik hoop dat ik niemand vergeten ben. Mocht dit toch het geval zijn, dan is dit te wijten aan de chaotische drukte in de eindfase van het afronden van mijn proefschrift, want het moge duidelijk zijn dat een proefschrift nooit helemaal af is. Daarnaast wil ik, waarmee ik dit dankwoord ben begonnen, mijn Mereltje nogmaals bedanken voor de steun en liefde van de afgelopen jaren.

Marchel 
Marhe! Gomelink 
Marchel Gorselink 